\title{
Ketogenic diet therapies
}

Citation for published version (APA):

Lambrechts, D. A. J. E. (2016). Ketogenic diet therapies: treatment for children and adults with refractory epilepsy . [Doctoral Thesis, Maastricht University]. https://doi.org/10.26481/dis.20160601dl

Document status and date:

Published: 01/01/2016

DOI:

$10.26481 /$ dis.20160601dl

Document Version:

Publisher's PDF, also known as Version of record

\section{Please check the document version of this publication:}

- A submitted manuscript is the version of the article upon submission and before peer-review. There can be important differences between the submitted version and the official published version of record.

People interested in the research are advised to contact the author for the final version of the publication, or visit the DOI to the publisher's website.

- The final author version and the galley proof are versions of the publication after peer review.

- The final published version features the final layout of the paper including the volume, issue and page numbers.

Link to publication

\footnotetext{
General rights rights.

- You may freely distribute the URL identifying the publication in the public portal. please follow below link for the End User Agreement:

www.umlib.nl/taverne-license

Take down policy

If you believe that this document breaches copyright please contact us at:

repository@maastrichtuniversity.nl

providing details and we will investigate your claim.
}

Copyright and moral rights for the publications made accessible in the public portal are retained by the authors and/or other copyright owners and it is a condition of accessing publications that users recognise and abide by the legal requirements associated with these

- Users may download and print one copy of any publication from the public portal for the purpose of private study or research.

- You may not further distribute the material or use it for any profit-making activity or commercial gain

If the publication is distributed under the terms of Article $25 \mathrm{fa}$ of the Dutch Copyright Act, indicated by the "Taverne" license above, 


\section{Ketogenic diet therapies}

Treatment for children and adults with refractory epilepsy 
Copyright (C) 2016 by Danielle Lambrechts. All rights reserved.

ISBN: 978-94-028-0140-8

Cover: Rachel van Esschoten, DivingDuckDesign.nl

Layout: Tiny Wouters

Printed by: Ipskamp Drukkers

The printing of this thesis was financially supported by Academic Center for Epileptology; department reseach and development, EISAI BV, LivaNova, Nutricia Advanced Medical Nutrition, UCB Pharma BV, University of Maastricht; Faculty of Health, Medicine \& Life Sciences. 


\title{
Ketogenic diet therapies \\ Treatment for children and adults with refractory epilepsy
}

\author{
PROEFSCHRIFT \\ Ter verkrijging van de graad van doctor aan de Universiteit Maastricht, \\ op gezag van de Rector Magnificus, Prof. dr. L.L.G. Soete, \\ volgens het besluit van het College van Decanen, \\ in het openbaar te verdedigen \\ op woensdag 1 juni 2016 om 14.00 uur
}

door

Danielle Ann Josée Eduard Lambrechts 
Promotores

Prof. dr. H.J.M. Majoie

Prof. dr. J.S.H. Vles

Prof. dr. A.P. Aldenkamp

\section{Copromotor}

Dr. A.J.A. de Louw

\section{Beoordelingscommissie}

Prof. dr. R.J. van Oostenbrugge (voorzitter)

Dr. C.E. Catsman-Berrevoets (ErasmusMC-Sophia)

Prof. dr. L. Lagae (UZ Leuven)

Prof. dr. R. Ponds

Prof. dr. L.J.I. Zimmermann 
Voor Joren en Bram

Blijf steeds geloven in het goede en de kracht van positieve gedachten 



\section{Contents}

Chapter 1 General introduction

Chapter 2 The MCT-ketogenic diet as a treatment option in refractory

childhood epilepsy: a prospective study with 2-year follow-up Epilepsy \& Behavior 2015;51:261-6

Chapter 3 Ketogenic diet effects on cognition, mood, and psychosocial adjustment in children

Acta Neurol Scand 2013;127:103-8

Chapter 4 The ketogenic diet as a treatment option in adults with chronic refractory epilepsy: efficacy and tolerability in clinical practice Epilepsy \& Behavior 2012;23:310-4

Chapter 5 Research into the (cost-)effectiveness of the ketogenic diet among children and adolescents with intractable epilepsy: design of a randomized controlled trial BMC Neurology 2011;11:10

Chapter 6 A randomized controlled trial of the ketogenic diet in refractory childhood epilepsy Acta Neurol Scand. 2016 Mar 29. doi: 10.1111/ane.12592.

Chapter 7 An economic evaluation of the ketogenic diet versus care as usual in children and adolescents with intractable epilepsy: an interim analysis Epilepsia 2016;57:41-50

Chapter 8 Blood beta-hydroxybutyrate correlates better with seizure reduction due to ketogenic diet than do ketones in the urine Seizure 2010;19:36-9

Chapter 9 Can an early 24-hour EEG predict the response to the ketogenic diet? A Prospective study in 34 children and adults with refractory epilepsy treated with the ketogenic diet Seizure 2014;23:468-74

Chapter 10 A prospective study on changes in blood levels of cholecystokinin-8 and leptin in patients with refractory epilepsy treated with the ketogenic diet Submitted 
Chapter 11 General discussion

Chapter 12

191

Summary

193

Samenvatting

199

Valorisatie

205

Dankwoord

213

List of publications

221

Curriculum Vitae

229 



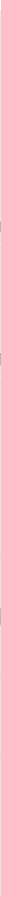




\section{Chapter 1}

General introduction

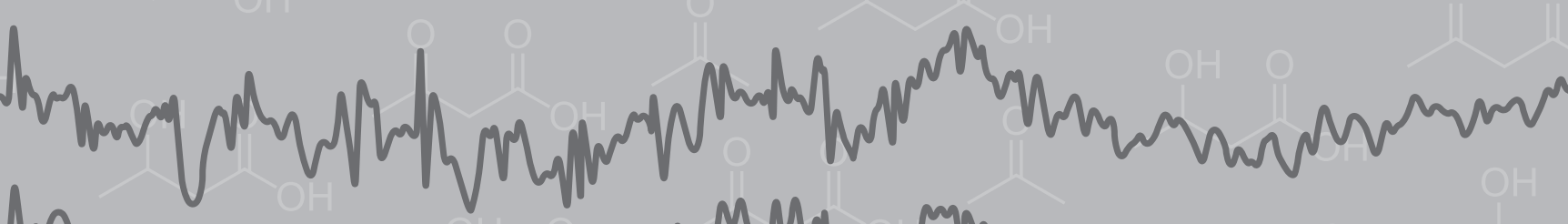

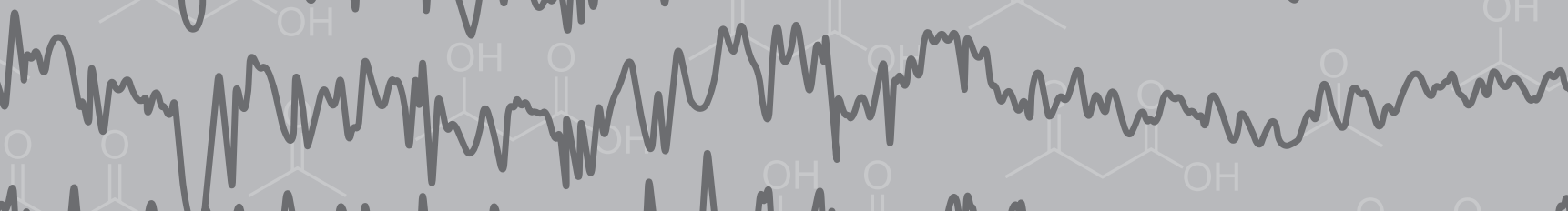

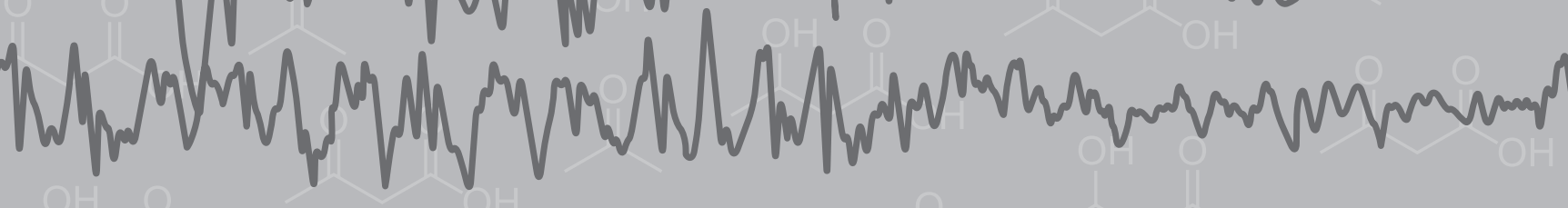


Chapter 1

1 


\section{General introduction}

This chapter presents background information on epilepsy and the treatment options, followed by the essentials of health technology assessment and economic evaluations.

The history and types of ketogenic diet therapies are explained, and current recommendations on the use of the ketogenic diet (KD) are outlined.

Finally, the chapter concludes with a list of research questions which are addressed in this thesis.

\section{Epilepsy}

Epilepsy is not a single disease but a collection of conditions with a wide range of underlying etiologies and pathologies. ${ }^{1}$ It is a disease of the brain defined by any of the following conditions: I) At least two unprovoked (or reflex) seizures occurring $>24$ hours apart, II) one unprovoked (or reflex) seizure and a probability of further seizures similar to the general recurrence risk (at least 60\%) after two unprovoked seizures, occurring over the next 10 years, or III) diagnosis of an epilepsy syndrome. ${ }^{2}$

Seizures are the clinical manifestation of abnormal, excessive and synchronous excitation of neuronal populations. Depending on the pattern of neuronal involvement, the symptomatology of a seizure may consist of a wide range of sudden and transitory phenomena, which may include alterations of consciousness, and/or motor, sensory, autonomic or psychic events. ${ }^{1}$

Seizures can be classified by location of onset of epileptic discharges. Generalized means both cerebral hemispheres are (rapidly) involved. In focal seizures, onset is within a circumscribed region of one of the cerebral hemispheres. Sometimes the location of onset remains unknown. A further classification of focal seizures can be made according to the degree of impairment of consciousness or awareness.

Recently, the classification system of seizures and epilepsy syndromes has been altered. For instance, complex partial seizures are now referred to as focal seizures with impairment of consciousness. Etiologies of epilepsy were subdivided into symptomatic, cryptogenic and idiopathic, terms which, in the classification proposal of Berg, have been replaced by "structural or metabolic epilepsy", "epilepsy of unknown cause" and "genetic". ${ }^{3-5}$

An EEG may be used to help determine seizure type and epilepsy syndrome in children, young people and adults in whom epilepsy is suspected. ${ }^{6}$ Cerebral electrical activity in the form of local field potentials can be recorded with a surface EEG (electro-encephalography). "Epileptic" neurons can synchronize with other neurons in the vicinity or at a distance in a functional network. When aligning, cortical neurons simultaneously exhibit a depolarization shift, producing a typical graphic element 
(spike-(and slow-)wave complex) in the EEG. EEG discharges registered during an epileptic seizure are ictal discharges; discharges without the appearance of a seizure are interictal epileptiform discharges (IEDs). ${ }^{7}$ The occurrence of IEDs in the EEG confirms the diagnosis of epilepsy with a high probability. ${ }^{8}$ Interictal epileptiform discharges are helpful in defining the epilepsy syndrome and provide information on epilepsy severity.

Worldwide, about 65 million people are estimated to have epilepsy. ${ }^{9}$ It is the most common serious neurological disorder. ${ }^{10}$ Incidence varies greatly with age, with high rates in early childhood, low rates in early adult life and an escalating second peak in people aged over 65 years. ${ }^{1}$ There are significant differences in incidence and prevalence rates between the advanced and the developing countries. 9,11

In Europe, the prevalence of epilepsy is $0.6 \% .{ }^{12}$ This means that in The Netherlands (16.9 million people), approximately 101,000 people have epilepsy, the annual incidence being 6,400 patients. About 2,000 of them (30\%) will suffer from refractory epilepsy.

\section{Treatment and refractory epilepsy}

Following the second unprovoked seizure, or after the first seizure with high probability of recurrence, anti-epileptic drug treatment is initiated.

Its goal is to modify the bursting properties of neurons and to reduce hypersynchronization, thereby inhibiting the spread of abnormal neuronal firing. ${ }^{13}$

The study by Mohanraj and Brodie showed that overall response rates with the first, second and third treatment schedules were 50.4, 10.7 and $2.7 \%$, respectively, with only $0.8 \%$ patients responding optimally to further drug trials. ${ }^{14}$

Several synonyms for "refractory epilepsy" are used in the literature; e.g. intractable epilepsy, therapy-resistant epilepsy, drug-resistant epilepsy, pharmaco-resistant epilepsy, all describing the same condition.

Since 2010, drug-resistant epilepsy has been defined as failure of adequate trials of two tolerated, appropriately chosen and administered antiepileptic drug schedules (either as monotherapies or in combination) to achieve sustained freedom from seizures. $^{15}$

Although many newer antiepileptic drugs (AEDs) have been developed in recent years, pharmaco-resistance remains a major therapeutic challenge, with more than $30 \%$ of patients not becoming seizure-free. ${ }^{16}$ In children, approximately one in 10 has intractable seizures in the early course of the disorder. Intractability can, however, develop over time ${ }^{5}$ and during follow-up, 3 out of 10 children were found to become intractable at some stage. ${ }^{17}$

Epilepsy surgery is an effective and safe alternative form of therapy for selected patients with refractory partial epilepsy. ${ }^{18}$ Depending on the epilepsy syndrome and 
the ability to define clearly and resect completely the epileptogenic zone, $30-85 \%$ of epilepsy patients who undergo surgery remain seizure-free. ${ }^{19}$

Patients with drug-resistant epilepsy who are not suitable for epilepsy surgery could benefit from vagus nerve stimulation (VNS) or a ketogenic diet (KD). VNS is a pacemaker-like device that is implanted subcutaneously into the upper part of the chest. The spiral electrode is wrapped around the left vagus nerve. The connector end of the lead is connected to the generator. ${ }^{20}$ Once the stimulator is activated, electrical pulses will be generated at regular intervals depending on the anticonvulsant effect and on the patient's tolerance.

\section{The ketogenic diet therapies}

The earliest reports of the effects of starvation on epilepsy date back to the fifth century when Hippocrates described a man whose seizures were cured by abstaining from all food and drink. First reports in the medical literature were by Guelpa and Marie who described the effect of complete fasting on the cessation of seizures. This was subsequently reported again in 1921 by Geyelin, stating that 20 of 26 fasting patients had improved seizure control, 2 of whom remained seizure-free for more than a year. It was suggested by Wilder that a diet high in fat and low in carbohydrate may mimic the ketotic effect: driving the body to utilize the fat in the diet, thus generating ketosis. ${ }^{21}$ The beneficial effects of the KD were confirmed throughout the 1930s, then largely forgotten, as first phenytoin and then other anticonvulsants became available. Interest in the KD was re-awakened during the early 1990 s. $^{22}$

Many theories have been suggested to explain the antiepileptic effect of the KD, ${ }^{22,23}$ but the exact mechanism is still unclear. Hypotheses range from 1) ketone bodies as direct anti-seizure agents to 2) changes in neurotransmitter systems as well as ion channel regulation, 3) enhancement of cellular bioenergetics and mitochondrial function, 4) glycolytic restriction, 5) direct inhibitory actions of fatty acids, 6) reduction in oxidative stress and 7) enhancement of tricarboxylic acid cycle function. ${ }^{24}$ Currently, the focus is on changes in energy metabolism. ${ }^{23,25}$ A variety of (neuro-)peptides may be altered which could have a significant influence on neuronal excitability. ${ }^{25,26}$

\section{Different types}

The KD is a high-fat, low carbohydrate, normocaloric diet that mimics the metabolic state of fasting. During a prolonged fast, body energy requirements are met by lipolysis and ß-oxidation of fatty acids rather than by the breakdown of carbohydrates. Thus, the KD maintains an anabolic nutritional state in a metabolic situation of fasting. Any diet providing nutritional fat for the generation of ketones that serve as an alternative fuel to body tissues can be called "ketogenic". 
Four different types exist, of which mixed forms can be used.

The focus of the KD studies in this thesis is the MCT-diet, although some patients were treated with the classical KD or a mixed form. Only a few adult patients were treated with the MAD. The low glycemic index diet treatment (LGIT) is beyond the scope of this thesis.

\section{The classical ketogenic diet}

Most studies report the use of the classical KD which has been used since the $1920 \mathrm{~s}$ and which is based on a ratio of fat to carbohydrate and protein combined, of 3:1 or $4: 1 .^{27-29}$ In the classical KD, long-chain triglycerides (LCTs) are used; $90 \%$ of energy intake consists of fat. In a 4:1 ratio, there are 4 grams of fat for every 1 gram of protein and carbohydrate combined. Protein is provided to meet dietary reference intake, which is approximately $1 \mathrm{~g}$ per kilogram of body weight. Carbohydrates complete the remaining allowance of the ratio.

\section{The MCT ketogenic diet}

A new variant of the KD that uses medium-chain triglycerides (MCT) as an alternative fat source was introduced in the 1970s. The major goal was greater palatability. ${ }^{30,31}$ The MCT diet yields more ketones per kilocalorie of energy provided than long-chain triglycerides (LCT); they are absorbed more efficiently, and are carried directly from the digestive system to the liver by the portal vein. Following hepatic tissue uptake there are other differences: long chain fat is first bound to carnitine, which is required for transport across the mitochondrial membrane where beta-oxidation and ketobody synthesis occurs. Such a carnitine shuttle system is not required for medium chain fatty acids, which enter the mitochondria directly. ${ }^{26}$ The increased ketogenic potential of MCT means that less total fat is needed in the MCT diet, thus enabling the inclusion of more carbohydrate and protein. The MCT diet contains $71 \%$ fat, $60 \%$ of which is $\mathrm{MCT}^{30}$

A modified MCT- diet, called the Radcliff diet, was developed in 1989; it provides only $30 \%$ of energy from MCT and $30 \%$ from $\mathrm{LCT}^{32}$

\section{The modified Atkins diet (MAD)}

The first report on the use of the Atkins diet as therapy for intractable epilepsy was published in 2003; the first report on the modified Atkins diet in 2006. ${ }^{33,34}$ The MAD is similar in composition to the classical KD; the ketogenic ratio is approximately $1: 1$. The initial daily carbohydrate consumption is approximately $10 \mathrm{~g}$, with a planned increase to $15-20 \mathrm{~g} /$ day after 1-3 months. There are, however, no limitations on protein, fluids and calories, making meal planning easier. ${ }^{35}$ 


\section{The low glycemic index diet treatment (LGIT)}

The first report on the use of the LGIT for treatment of intractable epilepsy was published in 2005. The LGIT allows liberalization of total daily carbohydrate intake to approximately 40-60 g/day, but regulates the type of carbohydrate, favoring those that produce relatively small changes in blood glucose (those with low glycemic indices $<50)^{36}$

\begin{tabular}{|l|c||c||c|}
\hline Dietary energy contribution* & Fat & Carbohydrate & Protein \\
\hline 'Healthy' recommendations & $30-35 \%$ & $50-55 \%$ & $15 \%$ \\
\hline Classical 4:1 ratio & $90 \%$ & $4 \%$ & $6 \%$ \\
\hline MCT & $71-75 \%$ & $15-19 \%$ & $10 \%$ \\
\hline MAD & $(30-60 \% \mathrm{MCT})$ & $6 \%$ & $30 \%$ \\
\hline LGIT & $64 \%$ & $10 \%$ & $30 \%$ \\
\hline
\end{tabular}

* Percentages in the table are based on references. ${ }^{22,33,36}$

\section{'Healthy' recommendations}

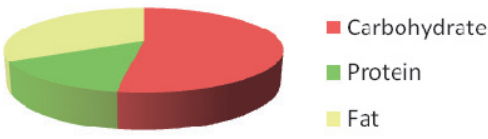

\section{Classical 4:1 ratio}

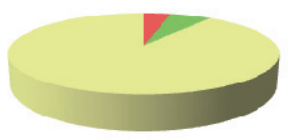

MCT

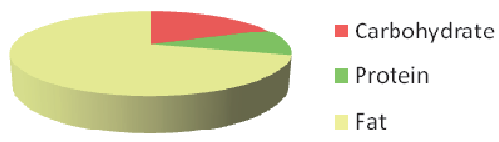

MAD

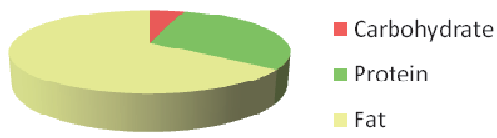

\section{Current recommendations on the use of the KD}

In 2009, the recommendations of the International Ketogenic Diet Study Group were published with the aim of producing a consensus statement regarding the clinical management of the KD.

The study group concluded that the KD should be strongly considered in a child, regardless of age or gender, in whom two to three anticonvulsant therapies had failed, and particularly in those with symptomatic generalized epilepsies. In specific epilepsy syndromes - Dravet syndrome, infantile spasms, myoclonic-astatic epilepsy, tuberous sclerosis complex - the KD could be offered earlier. The KD is probably only 
of limited benefit in children who are candidates for epilepsy surgery. KD is the treatment of first choice for two distinct disorders of brain metabolism: GLUT-1 deficiency syndrome and pyruvate dehydrogenase complex deficiency. ${ }^{35}$

The Dutch guideline for treatment of epilepsy advises that KD treatment be considered in children, and possibly in adults, with refractory epilepsy (i.e. two or more AED did not result in seizure control) in whom epilepsy surgery is not possible. ${ }^{37}$

\section{Health technology assessment and economic evaluation}

Health technology assessment (HTA) is a multi-disciplinary field of policy analysis that studies the medical, social, ethical and economic implications of development, diffusion and use of health technology. ${ }^{38}$

A frequently used tool in HTA research is an economic evaluation. In a trial-based economic evaluation, data on resource use and effectiveness are collected simultaneously. An economic evaluation covers a range of types used to investigate the costs and consequences of interventions, i.e. cost-effectiveness analysis (CEA) and cost-utility analysis (CUA). In CEA, the consequences of an intervention are measured in, for instance, seizure-free patients. In CUA, the consequences of an intervention are adjusted by health state preference scores or utility weights. The most common measure is the quality-adjusted life-year (QALY). ${ }^{39}$

The total annual costs of epilepsy in Europe are $€ 15.5$ billion. ${ }^{12}$ In The Netherlands, direct medical costs in 2005 were $€ 221$ million. ${ }^{40}$ In addition to the economic burden, epilepsy imposes a substantial burden on the patients themselves and their environment. Patients with uncontrolled epilepsy depend heavily on informal care (family and friends) and health care professionals. The high concentration of costs among refractory patients emphasizes the importance of seizure control and the costsaving potential of effective interventions. ${ }^{41} \mathrm{~A}$ formal assessment of cost-effectiveness in KD has, however, not yet been performed. 


\section{The present thesis}

In a tertiary epilepsy center, the reason for referral is often the demand for better treatment options for refractory epileptic seizures.

Bearing in mind the high prevalence of refractory epilepsy and the above-mentioned recommendations and guideline, the $K D$ is - in our opinion - only be used in the minority of patients who could potentially benefit from it.

The research questions addressed in this thesis are formulated below.

Research question 1: Is the ketogenic diet, especially the MCT-diet, an effective and well-tolerated treatment for children, adolescents and adults with refractory epilepsy?

Chapter 2 describes the efficacy and tolerability of treatment with the MCT and classical KD in 48 children with a 2-year follow-up.

Chapter 3 presents the effects on cognition, mood and psychosocial adjustment in 15 school-aged children and adolescents treated with the KD for at least six months.

Chapter 4 describes the efficacy and tolerability of treatment with the MCT and classical KD in 15 adults with refractory epilepsy, including effects on global cognitive performance, mood and quality of life.

Chapter 5 includes the design of the randomized controlled trial; research into the cost-effectiveness of the ketogenic diet among children and adolescents with intractable epilepsy.

Chapter 6 presents the short-term results on efficacy and tolerability of the randomized controlled trial.

Research question 2: Is the ketogenic diet a cost-effective treatment for children with refractory epilepsy?

Chapter 7 contains the economic evaluation of the KD versus care as usual concerning the first 4 months of the trial.

Research question 3: Are electro-encephalography (EEG) and specific laboratory investigations useful during treatment with the KD?

Chapter 8 answers the question: does blood beta-hydroxybutyrate correlate better with seizure reduction due to the KD than ketones in the urine in 33 children and adults. 
Chapter 9 answers the question: can an early 24-hour EEG predict the response to the KD in 34 children and adults treated with the KD for at least 6 months in the observational trial.

Chapter 10 describes the changes in serum levels of the neuropeptides, cholecystokinin-8 (CCK-8) and leptin, during treatment with the KD.

Chapter 11 contains the general discussion in which the main findings of this thesis are summarized. The overall effect of the ketogenic diet, with implications for clinical practise and future research, are discussed. 


\section{References}

1. Kwan P, Brodie MJ. Refractory epilepsy: mechanisms and solutions. Expert Rev Neurother 2006;6:397-406.

2. Fisher RS, Acevedo C, Arzimanoglou A, et al. ILAE official report: a practical clinical definition of epilepsy. Epilepsia 2014;55:475-82.

3. Proposal for revised clinical and electroencephalographic classification of epileptic seizures. From the Commission on Classification and Terminology of the International League Against Epilepsy. Epilepsia 1981;22:489-501.

4. Proposal for revised classification of epilepsies and epileptic syndromes. Commission on Classification and Terminology of the International League Against Epilepsy. Epilepsia 1989;30:389-99.

5. Berg AT, Shinnar S, Levy SR, Testa FM, Smith-Rapaport S, Beckerman B. Early development of intractable epilepsy in children: a prospective study. Neurology 2001;56:1445-52.

6. National Institute for Health and Clinical Excellence (NICE). The Epilepsies: the diagnosis and management of the epilepsies in adults and children in primary and secondary care, Nice Clinical Guideline 137. In: National Institue for Health and Clincal Excellence, 2012.

7. Speckman E, Elger C, Gorji A. Neurolophysiologic basis of EEG and DC potentials. In: Schomer DL, Da Silva FL, eds. Niedermeyer's electroencephalography: basic principles, clinical applications, and related fields, 6th ed ed. Philadelphia: Lippincott Williams \& Wilkins, 2011: 17-31.

8. Wirrell EC. Prognostic significance of interictal epileptiform discharges in newly diagnosed seizure disorders. J Clin Neurophysiol 2010;27:239-48.

9. Ngugi AK, Bottomley C, Kleinschmidt I, Sander JW, Newton CR. Estimation of the burden of active and life-time epilepsy: a meta-analytic approach. Epilepsia 2010;51:883-90.

10. MacDonald BK, Cockerell OC, Sander JW, Shorvon SD. The incidence and lifetime prevalence of neurological disorders in a prospective community-based study in the UK. Brain 2000;123:665-76.

11. Ngugi AK, Kariuki SM, Bottomley C, Kleinschmidt I, Sander JW, Newton CR. Incidence of epilepsy: a systematic review and meta-analysis. Neurology 2011;77:1005-12.

12. Pugliatti M, Beghi E, Forsgren L, Ekman M, Sobocki P. Estimating the cost of epilepsy in Europe: a review with economic modeling. Epilepsia 2007;48:2224-33.

13. Rogawski MA, Loscher W. The neurobiology of antiepileptic drugs. Nat Rev Neurosci 2004;5:553-64.

14. Mohanraj R, Brodie MJ. Diagnosing refractory epilepsy: response to sequential treatment schedules. Eur J Neurol 2006;13:277-82.

15. Kwan P, Arzimanoglou A, Berg AT, et al. Definition of drug resistant epilepsy: consensus proposal by the ad hoc Task Force of the ILAE Commission on Therapeutic Strategies. Epilepsia 2010;51:1069-77.

16. Hao X, Goldberg D, Kelly K, Stephen L, Kwan P, Brodie MJ. Uncontrolled epilepsy is not necessarily the same as drug-resistant epilepsy: differences between populations with newly diagnosed epilepsy and chronic epilepsy. Epilepsy Behav 2013;29:4-6.

17. Berg AT, Vickrey BG, Testa FM, et al. How long does it take for epilepsy to become intractable? A prospective investigation. Ann Neurol 2006;60:73-9.

18. Cascino GD. When drugs and surgery don't work. Epilepsia 2008;49 Suppl 9:79-84.

19. Rosenow F, Luders H. Presurgical evaluation of epilepsy. Brain 2001;124:1683-700.

20. Ben-Menachem E, Manon-Espaillat R, Ristanovic R, et al. Vagus nerve stimulation for treatment of partial seizures: 1. A controlled study of effect on seizures. First International Vagus Nerve Stimulation Study Group. Epilepsia 1994;35:616-26.

21. Cross JH. Dietary therapies--an old idea with a new lease of life. Seizure 2010;19:671-4.

22. Freeman J, Veggiotti P, Lanzi G, Tagliabue A, Perucca E. The ketogenic diet: from molecular mechanisms to clinical effects. Epilepsy Res 2006;68:145-80.

23. Schwartzkroin PA. Mechanisms underlying the anti-epileptic efficacy of the ketogenic diet. Epilepsy Res 1999;37:171-80.

24. Rho JM. How does the ketogenic diet induce anti-seizure effects? Neurosci Lett 2015.

25. Giordano C, Marchio M, Timofeeva E, Biagini G. Neuroactive peptides as putative mediators of antiepileptic ketogenic diets. Front Neurol 2014;5:63.

26. Rho JM, Stafstrom CE. The ketogenic diet: what has science taught us? Epilepsy Res 2012;100:210-7. 
27. Keene DL. A systematic review of the use of the ketogenic diet in childhood epilepsy. Pediatr Neurol 2006;35:1-5.

28. Lefevre F, Aronson N. Ketogenic diet for the treatment of refractory epilepsy in children: A systematic review of efficacy. Pediatrics 2000;105:E46.

29. Neal EG, Chaffe $\mathrm{H}$, Schwartz $\mathrm{RH}$, et al. A randomized trial of classical and medium-chain triglyceride ketogenic diets in the treatment of childhood epilepsy. Epilepsia 2009;50:1109-17.

30. Huttenlocher PR, Wilbourn AJ, Signore JM. Medium-chain triglycerides as a therapy for intractable childhood epilepsy. Neurology 1971;21:1097-103.

31. Huttenlocher PR. Ketonemia and seizures: metabolic and anticonvulsant effects of two ketogenic diets in childhood epilepsy. Pediatr Res 1976;10:536-40.

32. Schwartz RH, Eaton J, Bower BD, Aynsley-Green A. Ketogenic diets in the treatment of epilepsy: shortterm clinical effects. Dev Med Child Neurol 1989;31:145-51.

33. Kossoff EH, Krauss GL, McGrogan JR, Freeman JM. Efficacy of the Atkins diet as therapy for intractable epilepsy. Neurology 2003;61:1789-91.

34. Kossoff EH, McGrogan JR, Bluml RM, Pillas DJ, Rubenstein JE, Vining EP. A modified Atkins diet is effective for the treatment of intractable pediatric epilepsy. Epilepsia 2006;47:421-24.

35. Kossoff EH, Zupec-Kania BA, Amark PE, et al. Optimal clinical management of children receiving the ketogenic diet: recommendations of the International Ketogenic Diet Study Group. Epilepsia 2009;50:304-17.

36. Pfeifer $\mathrm{HH}$, Thiele EA. Low-glycemic-index treatment: a liberalized ketogenic diet for treatment of intractable epilepsy. Neurology 2005;65:1810-2.

37. Nederlandse Vereniging voor Neurologie. Epilepsie. Richtlijnen voor diagnostiek en behandeling [online]. Available at: http://epilepsie.neurologie.nl/cmssite/index.php. Accessed 01-10-2015.

38. Hailey D, Babidge W, Cameron A, Davignon L-A. HTA Agencies and Decision Makers. In: An INAHTA Guidance Document, 2010.

39. Drummond MF, Sculpher MJ, Torrance GW, O'Brien BJ, Stoddart GL. Methods for the economic evaluation of health care programmes. Oxford: Oxford university press, 2005.

40. Poos M, Smit J, Groen J, Kommer G, Slobbe L. Kosten van ziekten in Nederland 2005: zorg voor euro's8. (Cost of Illness in the Netherlands 2005), 2008.

41. Beghi E, Frigeni B, Beghi M, De Compadri P, Garattini L. A review of the costs of managing childhood epilepsy. Pharmacoeconomics 2005;23:27-45. 


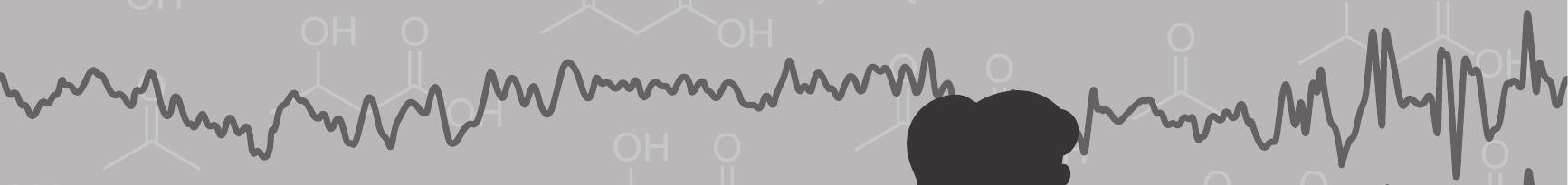

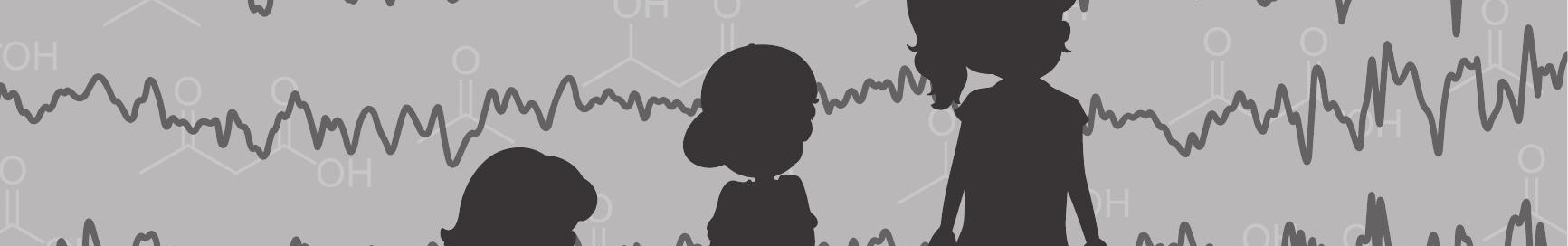




\section{Chapter 2}

\section{The MCT-ketogenic diet as a treatment option in refractory childhood epilepsy: \\ A prospective study with 2-year follow-up}




\section{Abstract}

The present study assessed the long-term (i.e., 24 months) efficacy of the ketogenic diet (KD) as an add-on therapy in children with refractory epilepsy, with focus on seizure frequency, seizure severity, and tolerability. Most patients were treated with the MCT-diet. At one and two years, $33 \%$ and $23 \%$, respectively, of the 48 included patients were still on the KD. After three months, one year, and two years of treatment, $16.7 \%$ of the patients were responders. The highest responder rate (i.e., 22.9\%) was seen at six and nine months of treatment. Of the fifteen patients with seizure clusters during baseline, $60 \%$ were responders after three months when looking at cluster reduction and most of them were not responders for the total seizure frequency. From three months of treatment onwards, most of the patients had a relevant decrease in seizure severity which was mainly related to the most severe seizure type.

Gastrointestinal dysfunction was often reported, especially in the first six weeks of treatment. Growth deceleration was present in $30 \%$ of the patients, and weight reduction in $15 \%$. Improved arousal was mentioned in $30 \%$ of patients. No patients developed ECG abnormalities or kidney stones. Increase in lipid profile was rare.

The KD is an effective therapy for children with therapy-resistant epilepsy. Effectiveness is reflected in the reduction of seizure frequency as well as in the reduction of seizure severity. After 6 months of treatment, it is obvious which patients are responders and tolerate the treatment well. Most of these patients will continue to benefit from the KD for a longer time. Long-term use of the diet was well tolerated. 


\section{Introduction}

Epilepsy is a common neurological disease, usually treated with anti-epileptic drugs (AEDs). Approximately $30 \%$ of patients, however, do not achieve remission with AEDs. ${ }^{1}$ Non pharmacological treatment options including the ketogenic diet (KD) can then be applied, especially when epilepsy surgery is not feasible.

The KD is a high-fat and low-carbohydrate diet of which there are various forms. The classical KD consists of long-chain triglycerides (LCTs), usually applied in a KD-ratio of 4:1 or 3:1 for [fat]:[proteins and carbohydrates]. Another well-known form of the KD is the MCT-diet, which consists mainly of medium-chain triglycerides (MCTs).

The randomized controlled trial of Neal et al. showed that the classical diet did not have any advantage over the MCT-diet in terms of efficacy and tolerability. ${ }^{2}$ In a recent Cochrane review, authors concluded that despite the heterogeneity, all trials showed that at least $38 \%$ of the patients had a $50 \%$ reduction in seizure frequency compared to controls at three months, and this response was maintained for up to a year. ${ }^{3}$ Follow-up periods of at least 12 months and preferably longer are advised to assess retention and long-term adverse effects. ${ }^{3}$ Until now, prospective long-term data in children, treated with the ketogenic diet, with a follow-up of more than 2 years are rare, ${ }^{4}$ are mostly about classical KD, or focused on specific side effects. ${ }^{5-7}$ In the present study, we assessed the long-term (i.e., 24 months) efficacy and tolerability of the KD as an add-on therapy in children with refractory epilepsy, focusing on seizure frequency, seizure clusters, and seizure severity.

\section{Methods}

This prospective observational clinical study was carried out within a tertiary referral center for epilepsy (Kempenhaeghe, Heeze, The Netherlands). Patients were included during the period January 2005 until September 2010. The study was approved by the Medical Ethics Committee according to Dutch Governmental Guidelines.

\section{Patients}

Children and adolescents, aged between 1 and 18 years with refractory epilepsy and ineligible for epilepsy surgery, were included. Refractory epilepsy was defined as seizures not adequately controlled by optimal treatment with $\geq 3$ AEDs. ${ }^{8}$ Patients were excluded if there were medical contraindications or the expectation that compliance with the diet was not possible because of severe behavioral or motivational problems. Medical exclusion criteria were fatty acid oxidation disorders, metabolic disorders causing hypoglycemia or metabolic acidosis, hyperlipidemia, hypercholesterolemia, cardiovascular disorders, long QT syndrome, malignancies, progressive cerebral lesions, degenerative diseases, and malnutrition. Treatment with topiramate, 
acetazolamide, or zonisamide was only excluded if combined with a positive family history of kidney stones or metabolic acidosis.

\section{Ketogenic diet}

The KD was introduced during a 2-week hospitalization at the Epilepsy Center. Usually, the MCT-diet was selected, but at subjects' request or if insurance did not refund the fat emulsions of the MCT-diet, the classical diet was used. When only tube feeding was given, a liquid form of the classical KD was used. Changes could be made to the diet because of side effects and other problems during the introduction phase.

The diet was introduced according to the Dutch guideline for KD. ${ }^{9}$ This guideline does not include a fasting period before introduction. In the classical diet, the fat intake was gradually built up over 4-5 days. In the MCT-diet, it takes 2-3 weeks to build up the fat to the required ratio. The building schedule starts with $10 \mathrm{~g}$ of MCT-fat: this amount is slowly increased to the calculated amount of MCT-fat. In addition, the LCTfat is increased in 4 days to the calculated amount of fat and then decreased in the same small steps as the MCT-fat is increased. Vitamin and mineral supplements were administered to avoid deficiencies.

The start of the diet was defined as the first change made to the daily nutrition of the patient. The end of the diet was defined as the first step in down titration to a regular diet. In case of a positive response, the KD could be continued for two years followed by tapering off. ${ }^{8}$ Patients were encouraged to continue the KD for at least 4 to 6 months before tapering off.

\section{Evaluation}

Evaluation was carried out at baseline, 6 weeks, and subsequently every 3 months after diet initiation. Seizure severity, side effects, and blood and urine samples were evaluated at each visit. Patients and/or caregivers were asked to record seizures continuously by means of a diary, starting 3 months before KD introduction. Reasons for KD discontinuation were recorded.

Total cholesterol, LDL cholesterol, and triglycerides were measured in the blood at each visit. The last sample during treatment was compared with baseline values.

Beta-hydroxybutyrate (BHB) was measured in the blood obtained by a finger prick. Urine samples were checked for ketone bodies (acetoacetic acid and acetone). At home, urine ketone levels were checked every day at similar times using a dipstick. If it proved impossible to obtain urine samples, BHB was measured three times a week. Levels of ketosis were recorded in the seizure calendar.

An ECG was performed before the start of the KD and subsequently every 6 months during treatment.

Side effects were evaluated by means of parental interview at each visit. 
At baseline and during the first 6 months on diet, changes in AED regime were only allowed if medically necessary. In case of adequate efficacy, the opportunity to reduce AEDs was given after 6 months.

Intention to treat (ITT) analysis was performed. The mean seizure frequency during the follow-up period was calculated per 3 months and compared to the baseline mean seizure frequency. When the mean seizure frequency was reduced by $\geq 50 \%$, the patient was defined as being a clinical responder. If seizure diaries were incomplete, the treating physician was asked to define the patient as a responder or non responder, using patients' and/or caregivers' information for qualitative data analysis. A seizure cluster ( $\geq 5$ seizures in a short time) was calculated as one seizure for the seizure frequency calculations. A subanalysis was made of patients with clusters during baseline.

Overall benefits of the KD in clinical practice were evaluated using retention rates. Seizure severity was assessed using the National Hospital Seizure Severity Scale (NHS3). ${ }^{10}$ The most severe seizure type for each patient was defined. Scores were calculated for the most severe seizure type and for all seizure types together. Values at each visit during the KD were compared with those at baseline. A difference of 2 points was estimated as being a clinically relevant improvement.

At each out-patient visit, growth and height were measured and processed using the growth analyzer version 3.5.197. Height-for-age and body mass index (BMI) were calculated and interpreted by the pediatrician of the KD team. A difference in standard deviation of $\geq 0.5$ for height-for-age and of $\geq 0.75$ for BMI was assumed to be clinically relevant. Only the results from patients who used the KD for at least 6 weeks were analyzed.

\section{Statistics}

Statistical analysis was performed using SPSS 21.0 for Windows. Seizure reduction data were analyzed using Wilcoxon's signed-rank test for nonparametric data (twotailed). Correlations between seizure reduction and blood BHB, urinary ketones, and demographic characteristics were investigated using Pearson's or Spearman's rank correlation test, depending on the measurement level and the distribution of the data. Significance level was set at $p<0.05$.

\section{Results}

Table 2.1 summarizes the main demographic and clinical characteristics at baseline. Forty-eight patients with a mean age of 7.8 years were included. Six patients were older than 13 years when the KD was initiated. Tonic-clonic, tonic, myoclonic, and focal seizures with impaired consciousness were the dominant seizure types, each present in $58.3 \%$ of the patients. Two patients were previously treated with vagus 
nerve stimulation (VNS); in one patient, VNS was activated during the KD. The MCTdiet was the predominant diet type (79\%). In 7 patients with a percutaneous gastrostomy tube (PGT), the liquid classical ketogenic diet was used exclusively. In 9 other patients with a PGT, the KD was partially given through PGT. During the treatment, the MCT-diet was changed into a mixed form of MCT and the classical diet in 9 patients, and in 5 patients, MCT-diet was replaced by the classical KD.

Table 2.1 Demography and clinical characteristics.

\begin{tabular}{|c|c|c|c|c|}
\hline & Mean & Min-max & $\mathrm{N}=48$ & $(\%)$ \\
\hline \multicolumn{5}{|l|}{ Gender } \\
\hline Male & & & 32 & $(66.7)$ \\
\hline Female & & & 16 & (33.3) \\
\hline \multicolumn{5}{|l|}{ Age (years;months) } \\
\hline At KD initiation & $7 ; 8$ & $1 ; 3-17 ; 5$ & & \\
\hline$\leq 5$ years & & & 18 & $(37.5)$ \\
\hline$>5-\leq 10$ years & & & 12 & $(25.0)$ \\
\hline$>10-\leq 15$ years & & & 15 & (31.3) \\
\hline$>15-\leq 18$ years & & & 3 & $(6.2)$ \\
\hline At seizure onset & $1 ; 7$ & $0 ; 0-11 ; 0$ & & \\
\hline Duration of epilepsy (years;months) & $6 ; 2$ & $1 ; 2-16 ; 3$ & & \\
\hline \multicolumn{5}{|l|}{ Total IQ } \\
\hline$<50$ & & & 32 & $(66.7)$ \\
\hline $50-69$ & & & 7 & (14.6) \\
\hline $70-99$ & & & 7 & (14.6) \\
\hline$\geq 100$ & & & 2 & (4.2) \\
\hline \multicolumn{5}{|l|}{ AEDs } \\
\hline Before KD & 7.5 & $4.0-14.0$ & & \\
\hline At KD initiation & 2.6 & $0-5$ & & \\
\hline \multicolumn{5}{|l|}{ Seizure Frequency at KD initiation } \\
\hline Daily seizures & & & 28 & (58.3) \\
\hline$\geq 1$ seizure a week & & & 19 & (39.6) \\
\hline$\geq 1$ seizure a month & & & 1 & $(2.1)$ \\
\hline Seizure types & 3.21 & $1-7$ & & \\
\hline \multicolumn{5}{|l|}{ Syndrome classification } \\
\hline Benign childhood epilepsy with centro-temporal spike & & & 1 & (2.1) \\
\hline Eyelid myoclonia with absences & & & 1 & (2.1) \\
\hline West syndrome & & & 1 & (2.1) \\
\hline Lennox-Gastaut syndrome & & & 1 & (2.1) \\
\hline Doose syndrome & & & 2 & (4.2) \\
\hline Dravet syndrome & & & 7 & $(14.6)$ \\
\hline Generalized epilepsies & & & 10 & $(20.6)$ \\
\hline Localisation-related epilepsies & & & 25 & $(52.2)$ \\
\hline \multicolumn{5}{|l|}{ Etiology } \\
\hline Genetic & & & 16 & (33.3) \\
\hline Structural & & & 19 & (39.6) \\
\hline Unknown & & & 13 & (27.1) \\
\hline \multicolumn{5}{|l|}{ Diet type } \\
\hline $\mathrm{MCT}$ & & & 38 & (79.2) \\
\hline Classical & & & 9 & (18.8) \\
\hline Mixed & & & 1 & (2.1) \\
\hline PGT & & & 16 & (33.0) \\
\hline
\end{tabular}

$\mathrm{N}=$ number; Min-max=minimum-maximum; $\mathrm{KD}=$ ketogenic diet; $\mathrm{AED}=$ anti-epileptic drug; $\mathrm{PGT}=$ percutaneous gastrostomy tube. 


\section{Retention rate}

Eleven patients (23\%) discontinued the diet within the first 3 months after initiation, 4 of whom discontinued before 3 weeks of treatment (see Figure 2.1). At 1 and 2 years, $16(33 \%)$ and $11(23 \%)$ patients, respectively, were still on the KD. Of these 11 patients, 4 are still maintaining the diet after 5 to 8 years.

The mean time on the KD during the 2-year study period was 10.25 months (range: 0.5-24 months).

Reasons for discontinuation before three months were side effects, problems with compliance, and increased seizure frequency or severity. Discontinuation after three months of treatment was mainly caused by lack of efficacy, although problems with compliance and side effects were still an issue. Two patients were lost to follow-up after treatment was transferred to the referring hospital.

In one patient, the KD was stopped acutely after 6 months of treatment because of gastric perforation and peritonitis due to complications of PGT replacement. One patient, treated for 9 months, died due to bilateral pneumonia. This severely disabled child suffered from pneumonia many times before treatment with the KD, which makes the diagnosis of lipid pneumonia unlikely. Another patient died after 6 months of treatment due to the complications of a convulsive status epilepticus.

After 1 year of treatment, most patients continued the diet for a longer time. Two patients started to taper off a month before 2 years of treatment because of practical reasons.

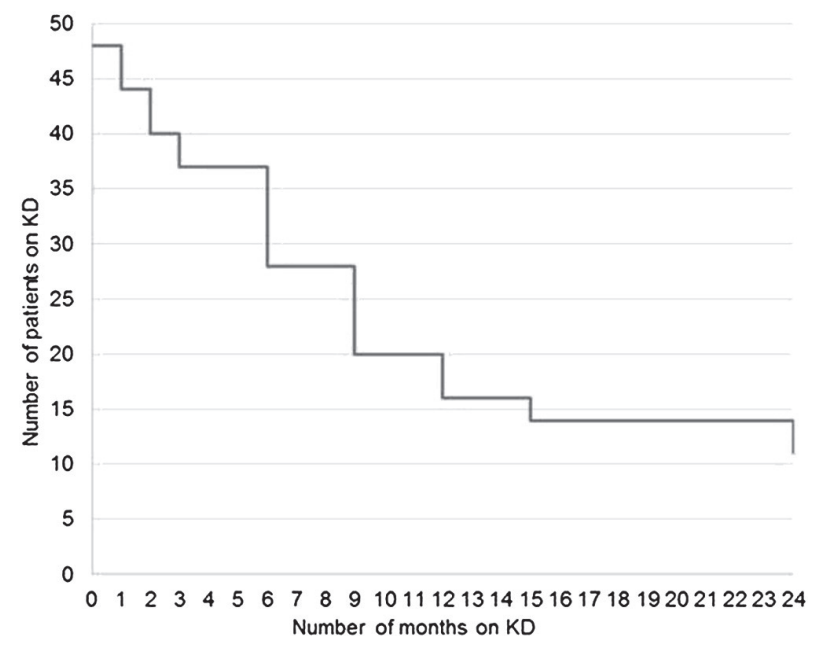

Figure 2.1 Retention curve, number of patients on KD during study period. 


\section{Seizure frequency}

Seizure outcome is reported in Table 2.2. Information on 48 patients was obtained.

In 35 patients, data were quantitatively analyzed. Highest responder rate was seen at 6 and 9 months of treatment, with 11 patients (22.9\%) being responders.

There was a statistically significant decrease in the mean seizure frequency at all time points $(p<0.05)$, except for the 12 -month visit $(p=0.056)$.

In 13 patients, qualitative data analysis was performed because of incomplete seizure diaries. Of these 13 patients, 4 continued the KD at 6 months, 1 of whom was a responder. After 12 to 24 months, only 2 patients continued the diet. One of these was seizure-free.

Table 2.2 Number of responders in ITT analysis and number of responders for cluster reduction.

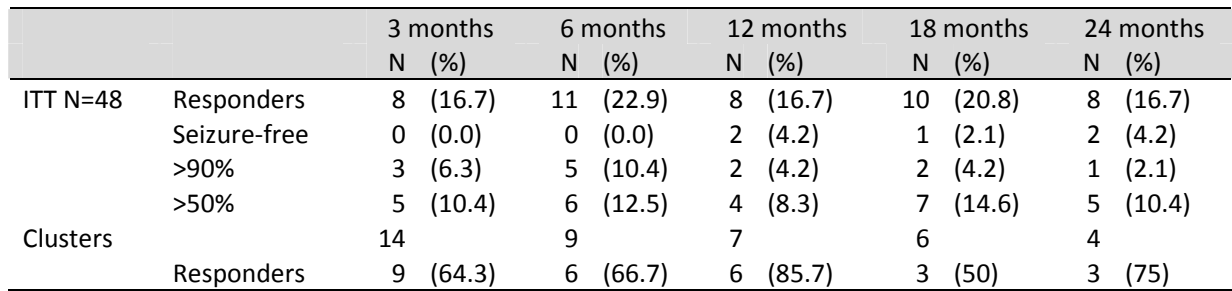

ITT=intention to treat; $\mathrm{N}=$ number; $\mathrm{KD}=$ ketogenic diet.

\section{Seizure clusters}

Of the 35 patients in whom quantitative analysis was performed, 15 had seizure clusters during baseline. Mean cluster frequency was 71.5 (min-max: 1-401). After 3 months, 9 patients (60\%) were responders for cluster reduction (see Table 2.2). Of these 9 responders, 7 patients were not responders for the total seizure frequency. At one year, $85.7 \%$ were responders, half of whom were not overall responders. At the endpoint of 2 years, $75 \%$ of patients with seizure clusters were responders, one of whom was not a responder for total seizure frequency.

Only one of the 6 patients aged over 13 years at the start of the KD was a responder and only for seizure clusters.

\section{Correlations}

There was no correlation between being a responder and gender, age at seizure onset, total intelligence quotient, syndrome classification, etiology of epilepsy, or number of different seizures at any time point during treatment. We found a negative correlation at 3 months between being a responder and duration of epilepsy $(p=0.049)$ and at 6 months between being a responder and age at introduction of the $K D(p=0.049)$. However, we did not find this correlation at other time points. 
At 9 months, a positive correlation with myoclonic seizures $(p=0.035)$ was present, but at other time points, no correlation with seizure type was found.

\section{Seizure severity}

Table 2.3 presents the number of patients showing an improvement in the NHS3 score compared to baseline. From 3 months of treatment, most of the patients on the KD had a relevant decrease in seizure severity, mainly related to the most severe seizure type.

Table 2.3 NHS3 score changes on total seizures and most severe seizure type at follow-up time points.

\begin{tabular}{|c|c|c|c|c|c|}
\hline $\begin{array}{l}\text { Time on the KD } \\
\text { Number and percentage }\end{array}$ & $\begin{array}{l}3 \text { months } \\
\mathrm{N}=20(\%)\end{array}$ & $\begin{array}{l}6 \text { months } \\
\mathrm{N}=16(\%)\end{array}$ & $\begin{array}{l}12 \text { months } \\
\mathrm{N}=10(\%)\end{array}$ & $\begin{array}{l}18 \text { months } \\
\mathrm{N}=9(\%)\end{array}$ & $\begin{array}{l}24 \text { months } \\
\mathrm{N}=7(\%)\end{array}$ \\
\hline Total seizures & & & & & \\
\hline $\begin{array}{l}\text { Score improvement } \\
\text { Most severe seizure type }\end{array}$ & $16(80)$ & $9(56)$ & $6(60)$ & $8(89)$ & $7(100)$ \\
\hline Score improvement & $13(65)$ & $8(50)$ & $5(50)$ & $7(78)$ & $6(86)$ \\
\hline
\end{tabular}

\section{AEDs}

On average, patients used 2.63 AEDs (range: $0-5$ ) at initiation of the KD. At discontinuation of the KD or at the end of the 2-year trial, the average number of AEDs was 2.46 (range: $0-5$ ). At the introduction of the KD, 30 patients used $\geq 3$ AEDs compared with 27 patients at the end of the KD. During the baseline, the dose of one AED was increased in a clinically relevant way in two patients who were not responders.

\section{Side effects}

Table 2.4 shows reported side effects during the KD.

Abdominal pain and vomiting were reported frequently during the first 6 months of treatment. Fatigue was increased in the first 6 weeks of treatment. Improved arousal was mentioned in $30 \%$ of patients throughout the treatment period. No menstrual irregularities were reported.

No ECG abnormalities appeared during the KD; in particular, no prolonged QTc-time was present. None of the patients developed kidney stones during treatment with the KD.

Total cholesterol, LDL cholesterol, and triglyceride values were available for 41 patients (Table 2.5). In 3 patients (7\%) with normal baseline values, there was a relevant increase in both total cholesterol and $\mathrm{LDL}$ cholesterol values during the $\mathrm{KD}$. In $2(5 \%)$ of these patients, triglycerides were also elevated. Their mean values for total cholesterol, LDL cholesterol, and triglycerides were, respectively, $1.8 \mathrm{mmol} / \mathrm{l}$, 
$1.6 \mathrm{mmol} / \mathrm{l}$ and $0.95 \mathrm{mmol} / \mathrm{l}$ higher than the highest normal level for their age and gender.

Duration of their treatment with the KD was 6 weeks, 21 months, and 24 months, respectively.

Complete measurements of height-for-age and BMI were available for 27 patients. In 8 children (30\%), height-for-age declined. These children were all on the KD for at least 6 months, 4 of them for 24 months. There was an unwanted weight reduction in 4 children (15\%) using the KD at least 3 months, 1 for 6 months and 2 for 24 months.

Table 2.4 Adverse effects.

\begin{tabular}{|c|c|c|c|c|c|c|c|}
\hline & $\begin{array}{l}\text { Baseline } \\
\mathrm{N}=48(\%)\end{array}$ & $\begin{array}{l}6 \text { weeks } \\
\mathrm{N}=44(\%)\end{array}$ & $\begin{array}{l}3 \text { months } \\
\mathrm{N}=38(\%)\end{array}$ & $\begin{array}{l}6 \text { months } \\
\mathrm{N}=28 \quad \%)\end{array}$ & $\begin{array}{c}12 \text { months } \\
\mathrm{N}=16(\%)\end{array}$ & $\begin{array}{l}18 \text { months } \\
\mathrm{N}=14(\%)\end{array}$ & $\begin{array}{r}24 \text { months } \\
\mathrm{N}=11(\%)\end{array}$ \\
\hline Abdominal pain & $0(0)$ & $7(16)$ & $3(8)$ & $4(14)$ & $0(0)$ & $0(0)$ & $0(0)$ \\
\hline Vomiting & $4(8)$ & $8(18)$ & $5(13)$ & $4(14)$ & $0(0)$ & $1(7)$ & $0(0)$ \\
\hline Diarrhea & $3(6)$ & $9(21)$ & $4(11)$ & $2(7)$ & $0(0)$ & $0(0)$ & $0(0)$ \\
\hline Constipation & 9 (19) & $9(21)$ & $5(13)$ & $1(4)$ & $1(6)$ & $1(7)$ & $1(9)$ \\
\hline Fatigue & $3(6)$ & $7(16)$ & $3(8)$ & $1(4)$ & $0(0)$ & $0(0)$ & $0(0)$ \\
\hline Improved arousal & $0(0)$ & $17(39)$ & $16(42)$ & $10(36)$ & $5(31)$ & $5(36)$ & $4(36)$ \\
\hline
\end{tabular}

Table 2.5 Mean fasting values of total cholesterol, LDL cholesterol and triglyceride ( $\mathrm{mmol} / \mathrm{l}$ ).

\begin{tabular}{lcccc}
\hline $\mathrm{N}=41$ & \multicolumn{2}{c}{ Baseline } & \multicolumn{2}{c}{ Last value on KD } \\
& & Min-max & Min-max \\
\hline Total cholesterol & 4.2 & $3.4-5.4$ & 4.9 & $2.5-7.6$ \\
LDL cholesterol & 2.0 & $1.1-3.5$ & 2.3 & $0.7-5.3$ \\
Triglyceride & 1.1 & $0.4-1.9$ & 1.2 & $0.4-3.2$ \\
\hline
\end{tabular}

\section{Ketosis}

Ketosis measured in the blood and urine is reported in Table 2.6. There was a significant correlation between being a responder and the single value of $\mathrm{BHB}$ in the capillary blood measured at the 12-, 15-, and 18-month visits (BHB $3.1 \mathrm{mmol} / \mathrm{l}$, $\mathrm{p}=0.024$; BHB $3.7 \mathrm{mmol} / \mathrm{l}, \mathrm{p}=0.001$; and $\mathrm{BHB} 2.8 \mathrm{mmol} / \mathrm{l}, \mathrm{p}=0.032$, respectively). No significant correlation was found for the mean of $\mathrm{BHB}$ or the mean of the urinary ketosis measured at home nor for the single value of ketones in urine measured at each out-patient visit throughout the treatment. 
Table 2.6 Ketosis in urine and blood ( $\mathrm{mmol} / \mathrm{l})$.

\begin{tabular}{|c|c|c|c|c|c|c|}
\hline \multirow{2}{*}{ BHB (serum) } & \multicolumn{2}{|c|}{$\begin{array}{l}3 \text { months } \\
\text { Min-max }\end{array}$} & \multicolumn{2}{|c|}{$\begin{array}{r}12 \text { months } \\
\text { Min-max }\end{array}$} & \multicolumn{2}{|c|}{$\begin{array}{r}24 \text { months } \\
\text { Min-max }\end{array}$} \\
\hline & & & & & & \\
\hline Single value & 2.6 & $0.2-6$ & 3.1 & $0.1-5.8$ & 2.8 & $0.2-4.6$ \\
\hline Mean & 2.4 & $0.4-4.8$ & 3.2 & $1.5-5.1$ & 4.3 & $3.5-5.1$ \\
\hline \multicolumn{7}{|c|}{$A c / A c A c$ (urine) } \\
\hline Single value & 5.5 & $0-16$ & 8.0 & $1.5-16$ & 10.0 & $0-16$ \\
\hline Mean & 6.5 & $0.6-15.3$ & 10.1 & $6-16$ & 7.4 & $6-9$ \\
\hline
\end{tabular}

$\mathrm{BHB}=$ beta-hydroxybutyrate; $\mathrm{Ac}=$ Acetone; $\mathrm{AcAc}=$ Acetoacetic acid.

\section{Discussion}

The main purpose of this study was to assess the long-term follow-up efficacy and tolerability of the KD as an add-on therapy in children with refractory epilepsy, focussing on seizure clusters and seizure severity.

The highest responder rate (i.e., 22.9\%) using an ITT analysis was present at 6 and 9 months of treatment. A substantial proportion of patients had a $>50 \%$ reduction in seizure clusters not reflected in the total responder rate. The majority of patients reported a relevant reduction in seizure severity during treatment with the KD. Twenty-three percent discontinued the diet within the first 3 months after initiation. After 1 and 2 years, 33\% and 23\% of patients, respectively, were still on the KD.

Compared to a recent Cochrane review, our results are less convincing. ${ }^{3}$ There may be numerous reasons for the difference in outcome.

Firstly, focal seizures have been associated with decreased likelihood of a favorable response to KD compared to other seizure types. ${ }^{11}$

Focal seizures with impaired consciousness were present in $58 \%$ of the patients in our study.

Besides this, there is some evidence that people with symptomatic generalized epilepsy may respond better to dietary treatment than those with idiopathic generalized epilepsy and partial epilepsy. ${ }^{12}$

Over $50 \%$ of patients in this study were classified as having localization-related epilepsy. In syndrome-specific cohort studies, patients with West syndrome, LennoxGastaut syndrome, Doose syndrome, and Dravet syndrome had the highest responder rates. ${ }^{13}$ A minority of children in our cohort were diagnosed with these syndromes.

Secondly, Freeman, as well as Coppola, described that patients under 8 years of age tend to have a better response to the ketogenic diet. ${ }^{14,15}$ In our study, almost $40 \%$ of children were over 10 years of age.

Dressler found that responders started with the KD after a significantly shorter duration of epilepsy than non responders. ${ }^{13}$ In our population, mean duration of epilepsy before treatment with the KD was 6.2 years (1.2-16.3, SD 4.8) which means a 
relatively long delay. We found a negative correlation with duration of epilepsy at 3 months; however, we did not find this correlation at other time points.

Thirdly, for patients with incomplete seizure diaries, qualitative analysis was performed. More patients in this subgroup discontinued the KD earlier. This subgroup exerts a negative impact on the efficacy of the total group.

Furthermore, in two patients, the dose of one AED increased during baseline. There is a possibility that this dose change resulted in baseline seizure decrease in these two children who were nonresponders.

Fourthly, mean ketone levels were within the accepted range, but for some patients, the values were lower. We need to consider the possibility that compliance was not always optimal.

Finally, the use of seizure diaries is accepted for clinical research and practice but has disadvantages. Subjects may pay more attention to seizures after changing treatment or may record 'false positive' events that are not seizures. ${ }^{16-18}$

The high dropout ratio before 3 months is in line with the results of Neal et al., where 25/125 patients (20\%) stopped before 3 months. At 12 months, 54\% were still using the diet compared with $33 \%$ in this study. ${ }^{2}$

Independent of the less convincing results on seizure reduction, there was a reduction in seizure clusters and a reduction in seizure severity in a substantial proportion of the patients. Previous literature about efficacy of the KD does not specify the effect on seizure clusters.

In this study, 64 to $86 \%$ of the patients with seizure clusters during baseline experienced $\geq 50 \%$ cluster reduction. Fifty to seventy-eight percent were not responders for the overall seizure count during the first year of treatment. Seizure severity is not often used as outcome measure of the KD. Hallböök et al. described a statistically significant decrease in seizure severity after 3 months of KD compared with the baseline measured as the mean of the NHS3 value. ${ }^{19}$

Gastrointestinal symptoms (abdominal pain, vomiting, and diarrhea) were the major adverse effects during the first 3 months of treatment, especially during the first 6 weeks of treatment. Nevertheless, reports of adverse effects during the 3-month baseline in the presented study revealed a high percentage of constipation and a few children suffering from vomiting and diarrhea before the start of dietary treatment.

Constipation is often reported in children using AEDs, especially when combined with low mobility.

Growth deceleration was present in $30 \%$ of the children; all were on the KD for at least 6 months. Relevant weight reduction was present in 15\% (4/27). In particular, decline in growth rate is of clinical importance, although there may be an overestimation due to difficulties in accuracy of measurement because of the existence of spasticity and contractures in many of these children. Nevertheless, our findings are in line with the previous literature. ${ }^{20-22}$

In 3 patients, there was a relevant increase in both total and LDL cholesterol values and in triglycerides in two during the KD. Previous studies showed an increase of $30 \%$ 
in both total cholesterol and TG during treatment with the KD. Sixty to eighty-five percent of children will have abnormal lipid levels at least temporarily. ${ }^{6}$ The use of medium-chain-TG as the source of fat in the KD appears to produce less dyslipidemia than the classical KD. ${ }^{6}$

Prolongation of the QT interval while using the KD is described in the literature. ${ }^{23,24}$ In this study, no ECG abnormalities, especially no prolonged QTc-time, were observed which is in line with more recent literature. ${ }^{25,26}$

In the literature, the prevalence of kidney stones ranges from $3 \%$ to $10 \%$ in children treated with the $\mathrm{KD}^{27}$ In this study, no patient developed kidney stones during treatment with the KD. Possible explanations for the different findings in our cohort are the consequent stimulation of adequate fluid intake. Furthermore, renal ultrasound was only performed in the case of clinical symptoms or persisting occult hematuria; asymptomatic renal calculi could be missed.

There was a significant correlation between being a responder and the single value of $\mathrm{BHB}$ in the capillary blood measured at the 12-, 15-, and 18-month visits. No significant correlation was found for the mean of $\mathrm{BHB}$ or the mean of the urinary ketosis measured at home, nor for the single value of ketones in urine measured at each outpatient visit.

It is remarkable that no significant correlation was present for the visits before 12 months of treatment in this study. The relationship between seizure control and ketosis is, however, still unclear. ${ }^{11}$

The added value of this study is the prospective design, with a long-term follow-up period of 2 years, including information on seizure frequency and seizure severity and paying special attention to seizure clusters on the one hand, and information about side effects on the other.

Limitations of the study include the nonrandomized design, uncontrolled selection of patients, and its limited sample size.

In conclusion, the KD is an effective therapy for children with therapy-resistant epilepsy. Effectiveness is reflected not only in the reduction of seizure frequency, but also in the reduction of seizure clustering and seizure severity. After 6 months of treatment, it is obvious which patients are having significant seizure reduction and tolerate the treatment well. Patients who do not experience any positive effect can be advised to taper off the KD. Most responders will continue to benefit from the dietary treatment for a longer period.

The most invalidating side effects, especially in the first 3 months of treatment, are gastrointestinal symptoms which can usually be reduced by fine-tuning the diet. 


\section{References}

1. Mohanraj R, Brodie MJ. Early predictors of outcome in newly diagnosed epilepsy. Seizure 2013;22:333-44.

2. Neal EG, Chaffe H, Schwartz RH, Lawson MS, Edwards N, Fitzsimmons G, et al. A randomized trial of classical and medium-chain triglyceride ketogenic diets in the treatment of childhood epilepsy. Epilepsia 2009;50:1109-17.

3. Levy RG, Cooper PN, Giri P. Ketogenic diet and other dietary treatments for epilepsy. Cochrane Database Syst Rev 2012;3:CD001903.

4. Sills MA, Forsythe WI, Haidukewych $D$, MacDonald A, Robinson M. The medium chain triglyceride diet and intractable epilepsy. Arch Dis Child 1986;61:1168-72.

5. Hemingway C, Freeman JM, Pillas DJ, Pyzik PL. The ketogenic diet: a 3- to 6-year follow-up of 150 children enrolled prospectively. Pediatrics 2001;108:898-905.

6. Kwiterovich Jr PO, Vining EP, Pyzik P, Skolasky Jr R, Freeman JM. Effect of a high-fat ketogenic diet on plasma levels of lipids, lipoproteins, and apolipoproteins in children. JAMA 2003;290:912-20.

7. Kim JT, Kang HC, Song JE, Lee MJ, Lee YJ, Lee EJ, et al. Catch-up growth after longterm implementation and weaning from ketogenic diet in pediatric epileptic patients.

Clin Nutr 2012;32:98-103.

8. Kossoff EH, Zupec-Kania BA, Amark PE, Ballaban-Gil KR, Christina Bergqvist AG, Blackford R, et al. Optimal clinical management of children receiving the ketogenic diet: recommendations of the International Ketogenic Diet Study Group. Epilepsia 2009;50:304-17.

9. van den Hurk TAM, van der Louw EJTM. Dieetbehandelingsrichtlijn ketogeen dieet voor kinderen (018 jaar) met refractaire epilepsie. Evidence-based handleiding voor een multidisciplinaire behandeling. Utrecht: UMC Utrecht; 2007.

10. O'Donoghue MF, Duncan JS, Sander JW. The National Hospital Seizure Severity Scale: a further development of the Chalfont Seizure Severity Scale. Epilepsia 1996;37:563-71.

11. Schoeler NE, Cross JH, Sander JW, Sisodiya SM. Can we predict a favourable response to ketogenic diet therapies for drug-resistant epilepsy? Epilepsy Res 2013;106:1-16.

12. Payne NE, Cross JH, Sander JW, Sisodiya SM. The ketogenic and related diets in adolescents and adults - a review. Epilepsia 2011;52:1941-8.

13. Dressler A, Stocklin B, Reithofer E, Benninger F, Freilinger M, Hauser E, et al. Longterm outcome and tolerability of the ketogenic diet in drug-resistant childhood epilepsy-the Austrian experience. Seizure 2010;19:404-8.

14. Freeman JM, Vining EP, Pillas DJ, Pyzik PL, Casey JC, Kelly LM. The efficacy of the ketogenic diet1998: a prospective evaluation of intervention in 150 children. Pediatrics 1998;102:1358-63.

15. Coppola G, Veggiotti P, Cusmai R, Bertoli S, Cardinali S, Dionisi-Vici C, et al. The ketogenic diet in children, adolescents and young adults with refractory epilepsy: an Italian multicentric experience. Epilepsy Res 2002;48:221-7.

16. Neal EG, Chaffe H, Schwartz RH, Lawson MS, Edwards N, Fitzsimmons G, et al. The ketogenic diet for the treatment of childhood epilepsy: a randomised controlled trial. Lancet Neurol 2008;7:500-6.

17. Fisher RS, Blum DE, DiVentura B, Vannest J, Hixson JD, Moss R, et al. Seizure diaries for clinical research and practice: limitations and future prospects. Epilepsy Behav 2012;24:304-10.

18. Sharma S, Sankhyan N, Gulati S, Agarwala A. Use of the modified Atkins diet for treatment of refractory childhood epilepsy: a randomized controlled trial. Epilepsia 2013;54:481-6.

19. Hallbook T, Lundgren J, Rosen I. Ketogenic diet improves sleep quality in children with therapyresistant epilepsy. Epilepsia 2007;48:59-65.

20. Neal EG, Chaffe HM, Edwards N, Lawson MS, Schwartz RH, Cross JH. Growth of children on classical and medium-chain triglyceride ketogenic diets. Pediatrics 2008;122:e334-40.

21. Spulber G, Spulber S, Hagenas L, Amark P, Dahlin M. Growth dependence on insulin-like growth factor-1 during the ketogenic diet. Epilepsia 2009;50:297-303.

22. Kim JT, Kang HC, Song JE, Lee MJ, Lee YJ, Lee EJ, et al. Catch-up growth after long-term implementation and weaning from ketogenic diet in pediatric epileptic patients.

Clin Nutr 2013;32:98-103. 
23. Best TH, Franz DN, Gilbert DL, Nelson DP, Epstein MR. Cardiac complications in pediatric patients on the ketogenic diet. Neurology 2000;54:2328-30.

24. Bank IM, Shemie SD, Rosenblatt B, Bernard C, Mackie AS. Sudden cardiac death in association with the ketogenic diet. Pediatr Neurol 2008;39:429-31.

25. Sharma S, Gulati S. The ketogenic diet and the QT interval. J Clin Neurosci 2012;19:181-2.

26. Doksoz O, Guzel O, Yilmaz U, Isguder R, Celegen K, Mese T. Dispersion durations of P-wave and QT interval in children treated with a ketogenic diet. Pediatr Neurol 2014;50:343-6.

27. Sampath A, Kossoff EH, Furth SL, Pyzik PL, Vining EP. Kidney stones and the ketogenic diet: risk factors and prevention. J Child Neurol 2007;22:375-8. 


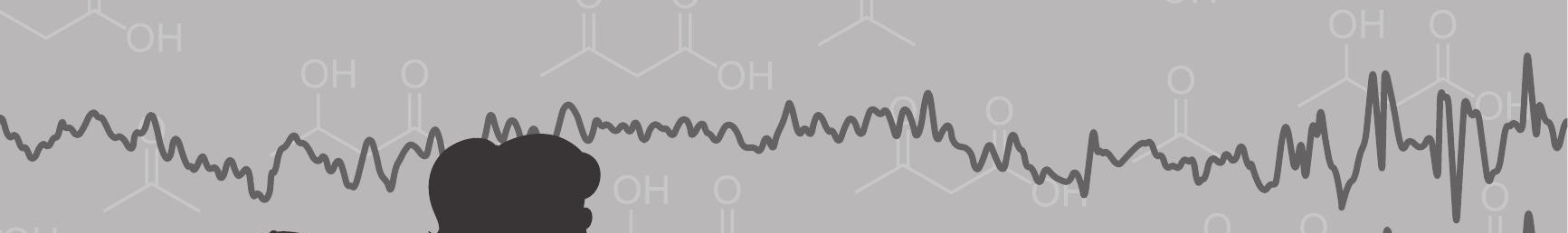

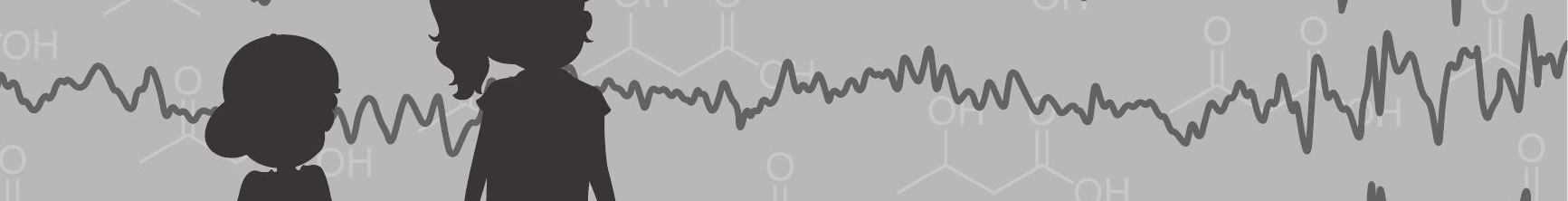

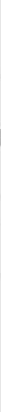




\section{Chapter 3}

\section{Ketogenic diet effects on cognition, mood, and psychosocial adjustment in children}

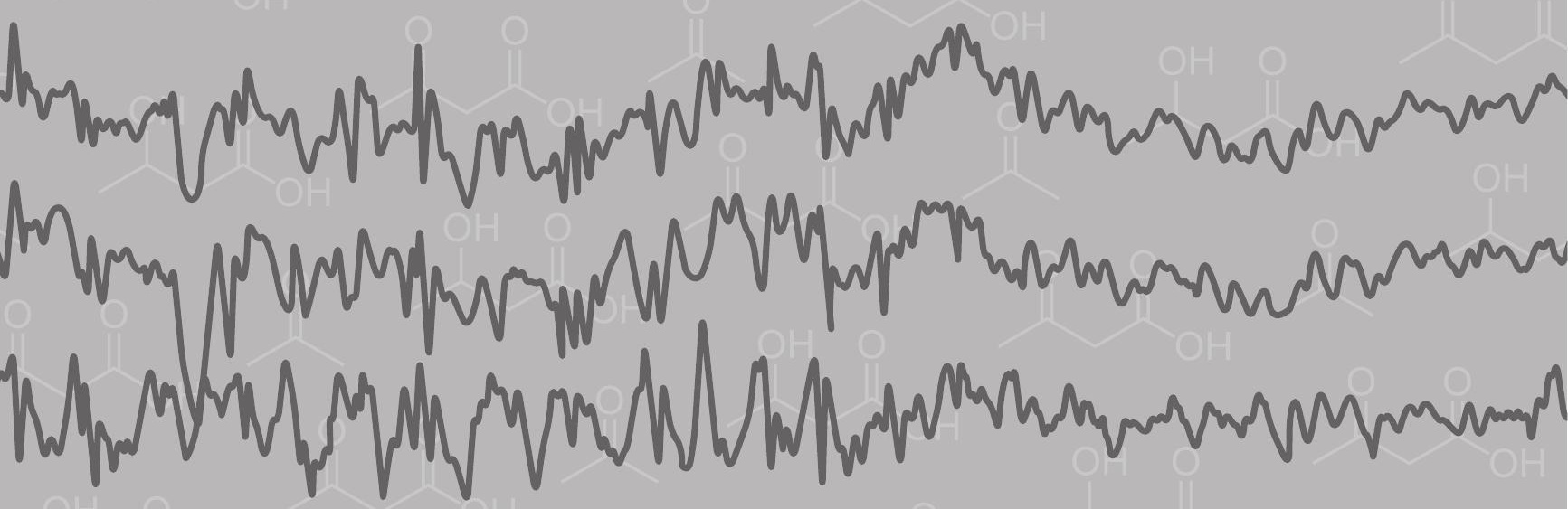

D.A.J.E. Lambrechts, M.J.M. Bovens, N.M. de la Parra, J.G.M. Hendriksen, A.P. Aldenkamp, H.J.M. Majoie 


\section{Abstract}

\section{Introduction}

The ketogenic diet (KD) is increasingly used for the treatment of refractory epilepsy. The aim of this study was to evaluate possible adverse effects of the diet on cognition, behavior, psychosocial adjustment, and quality of life in school-aged children and adolescents.

\section{Methods}

Fifteen subjects were assessed before diet initiation. After approximately 6 months, on diet treatment 11 patients (73\%) were reassessed. We used a combination of individually administered psychological tests for the children and parent report questionnaires.

\section{Results}

Five of 15 patients had a seizure reduction of more than $50 \%$. Cognition showed a small trend toward improvement in most patients. Psychosocial adjustment, on the other hand, showed small trends toward worsening. For mood, two areas showed a larger change, revealing more mood problems although this was not on a statistically significant level.

\section{Conclusion}

In this small group of children, there is no indication that the KD has a negative impact on cognition or social adaptation at short term. There is a tendency toward an increase in mood problems. 


\section{Introduction}

A broad spectrum of cognitive deficits and behavioral abnormalities are associated with epilepsy. ${ }^{1}$ There are several factors which can account for these problems, but one major cause are the side effects of the treatment. ${ }^{1}$

The ketogenic diet (KD) is used as an alternative to anti-epileptic drugs (AED) treatment in subjects with difficult-to-control epilepsy. It is a high-fat, lowcarbohydrate, low-protein diet that was developed in the 1920s and 'rediscovered' in the 1990s. ${ }^{2}$

Efficacy of the diet in children in terms of seizure reduction has been demonstrated in several studies. ${ }^{3-5}$ A recent randomized controlled trial showed that mean seizure frequency was reduced by $38 \%$ in children on the KD and increased by $37 \%$ in the control group. ${ }^{6}$

Besides seizure control, it is important to avoid tolerability problems especially in patients with refractory epilepsy. ${ }^{1}$ One of the common areas that represents tolerability problems is changes in cognition, behavior, mood, and alertness. ${ }^{7}$ Two prospective studies assessing changes in alertness and attention in children on KD treatment have been published, ${ }^{8,9}$ both of which found a significant improvement in attention. This improvement seemed to be independent of reduction in seizures or discontinuation of concomitant AEDs. However, both studies used parent-rated questionnaires and did not use cognitive tests performed by children themselves to assess attention. Besides, according to Neal et al., ${ }^{6}$ one of the most frequently reported side effects of KD treatment is lack of energy, which correlates with lack of attention. Overall behavior was not found to worsen or improve significantly in these studies, whereas behavioral outbursts are reported in children on KD treatment. Hallböök et al. ${ }^{9}$ found a significant improvement in quality of life (QOL) in children on KD treatment, confirmed by findings of Weber et al., ${ }^{10}$ who prospectively assessed changes in QOL. They reported improved QOL exclusively in 'responders' to the Atkins version of the KD. Besides these prospective studies, there have been several studies which provide retrospective or subjective information on cognitive, behavioral, and QOL-related effects of the KD. These indicated better cognitive functioning and, to a lesser extent, improved behavior in various numbers of children on KD treatment. ${ }^{7,11-17}$ Although these reports, taken together, suggest a satisfactory tolerability profile of KD, the lack of objective measurements does not exclude the possibility that KD treatment may negatively affect specific cognitive and behavioral functions.

The main purpose of this study is to objectively assess the adverse effects of the KD on cognition, mood, and social behavior in children with refractory epilepsy. For this, we used a combination of cognitive-oriented psychological tests performed by children themselves and by proxy questionnaires, assessing psychosocial adjustment and behavioral functioning. 


\section{Methods}

\section{Subjects}

Patients with refractory epilepsy aged 6 till 18 years were included between January 2005 and January 2009. Refractory was defined as suboptimal seizure control despite treatment with at least two adequately dosed AED and without option of surgical treatment. Patients remained on stable AED treatment for at least 3 months prior to starting the KD and during the first 6 months of treatment.

\section{Diet}

All patients were admitted to the clinic of the epilepsy center for a period of 2 weeks for initiation of the diet. Each patient embarked on the KD without prior fasting, according to the Dutch Guideline for the KD. ${ }^{18}$ For all subjects, the diet was calculated on an individual basis by the same dietician. In most patients, the median chain triglycerides (MCT) version of the diet was introduced, starting with a high content of long-chain triglycerides (LCT) and gradually replacing this by MCT-fat. After 3 weeks, the diet is built up to 70 En\% fat, 60 En\% MCT-fat, 10 En\% LCT-fat, 10 En\% protein, and $20 \mathrm{En} \%$ carbohydrates. Depending on the tolerance of the MCT-fat, the diet was adjusted to a classic version (3:1 or 4:1 ratio, fat:protein and carbohydrates) or a mixture of both types. In three children, the diet was administered via a gastric tube, using a classic version of the diet. Diets were fully supplemented with vitamins and minerals.

\section{Design}

During the 3 months prior to KD initiation and during the treatment phase, the parents kept a diary of seizure frequency and type. Effectiveness was defined as a $50 \%$ or more reduction in seizure frequency as compared to baseline. Different psychological tasks were administered to provide information on different cognitive outcomes: the Dutch version of the Peabody Picture Vocabulary test (global cognitive level), the FePsy neuropsychological test battery (attention), and Beery's Visual-Motor Integration (VMI) test (psychomotor functions). ${ }^{19-21}$ Parental report measurements were used to evaluate social behavior and mood with the Profile of Mood States (POMS) and the Personal Adjustment and Role Skills Scale, 3rd edition. ${ }^{22-25}$ Follow-up assessments on cognition and behavior were performed approximately 6 months after initiation of the KD. 


\section{Measures}

\section{Cognition}

An overall measurement of global cognitive level was performed with the Peabody Picture Test (Dutch version; PPVT-III-NL). This test assesses receptive vocabulary. ${ }^{9,26}$ The child has to point to one of four pictures corresponding to the examiner's stimulus word. Scores can be converted to age-equivalent standard scores. Good internal consistency is described. ${ }^{19}$ This test is considered to provide a good estimation of verbal intelligence. Additionally, the FePsy neuropsychological test battery has been conducted. It consists of several tasks. ${ }^{20}$ Simple reaction time measurements on either visual (a white square) or auditory stimuli assess speed information processing system and alertness. In the binary choice reaction test, the subject has to react in a different way to two dissimilar stimuli (a red square presented on the left side of the screen and a green square presented on the right side). This assesses the speed of central information processing. The Beery Developmental Test of VMI is a widely used paper-and-pencil test that assesses the extent to which individuals can integrate their visual and motor abilities. ${ }^{21}$ Subjects are asked to copy a developmental sequence of 24 geometric forms.

Mood

To assess mood, the by-proxy questionnaire POMS was used. It is a 65-item questionnaire that measures six transient, fluctuating affective mood states: depression/dejection, tension/anxiety, anger/hostility, vigor/activity, fatigue/inertia, and confusion/bewilderment. ${ }^{24}$ The respondent rates each item describing an emotional state on a 5-point scale ranging from 'not at all' to 'extremely'. The POMS was parent-rated in our study.

\section{Social behavior}

The Personal Adjustment and Role Skills Scale, 3rd edition (PARS-III), is a parent report questionnaire, developed to assess psychosocial adjustment in children with chronic physical illness. ${ }^{25}$ The 28 items relate to six psychosocial subscales: peer relations, dependency, hostility, productivity, anxiety/depression, and withdrawal, using a 4-point interval rating scale ranging from 'never or rarely' to 'always or almost always'. A higher score indicates better adjustment. ${ }^{27}$ Both reliability and validity proved to be adequate in children with a variety of chronic illnesses. ${ }^{27,28}$

\section{Statistical analysis}

Wilcoxon matched-pairs signed rank test was conducted to demonstrate changes in cognition, behavior and mood compared to baseline evaluations ( $p$-values were 
2 -sided). The level of significance was set at $p<0.05$. Data analysis was performed using SPSS (SPSS version 16.0; IBM, NY, USA).

\section{Results}

Fifteen school-aged children and adolescents were eligible for this study. Four (27\%) discontinued the diet before re-evaluation. Reasons for dropout were insufficient effectiveness in seizure control in three patients and increased seizure frequency with behavioral deterioration in one patient. Eleven subjects were re-evaluated after several months of the KD. The average age of the participants was $12 \pm 3.6$ years (range, 6-18), and the average age at onset of the epilepsy was $3.2 \pm 3.2$ (range, $0-11$ ). Patients were being treated with one to five AED at the time of starting with the KD treatment.

Demographic and clinical characteristics of the study population are shown in Table 3.1.

Five of 15 patients were responders with a reduction in seizure frequency of at least $50 \%$ after 6 months (intention to treat analysis).

The reasons for six patients remaining on the diet despite a decrease in seizure frequency of $<50 \%$ were improved alertness and some improvement in seizure control.

In most cases, AED treatment was not modified in the first 6 months of KD treatment. In one patient, it was possible to withdraw one AED.

Table 3.2 shows the results of the test procedure.

None of the cognitive tests showed a statistically significant change from baseline to endpoint. All tests but one showed a change in the positive direction, revealing small improvements in the magnitude of $1.6-8.7 \%$. One tests showed a decline, again in the modest range of $7.7 \%$.

For mood in general, differences between baseline performance and endpoint were larger and mostly (for five of the six subscales) in the negative direction revealing mood changes. None of the subscales, however, showed changes on a statistically significant level. The largest changes in the negative direction were for anger/hostility (negative change of $29 \%$ ) and on the other hand fatigue/inertia (negative change of $35 \%)$. In line with this also, vigor/activity showed a larger decline (11.8\%).

For social adaptation, the total score shows a decline of $3.1 \%$. In line with this, only one subscale shows a small improvement and one scale shows no change. The other scales show modest negative changes in the range of 1.9-7.5\%. 


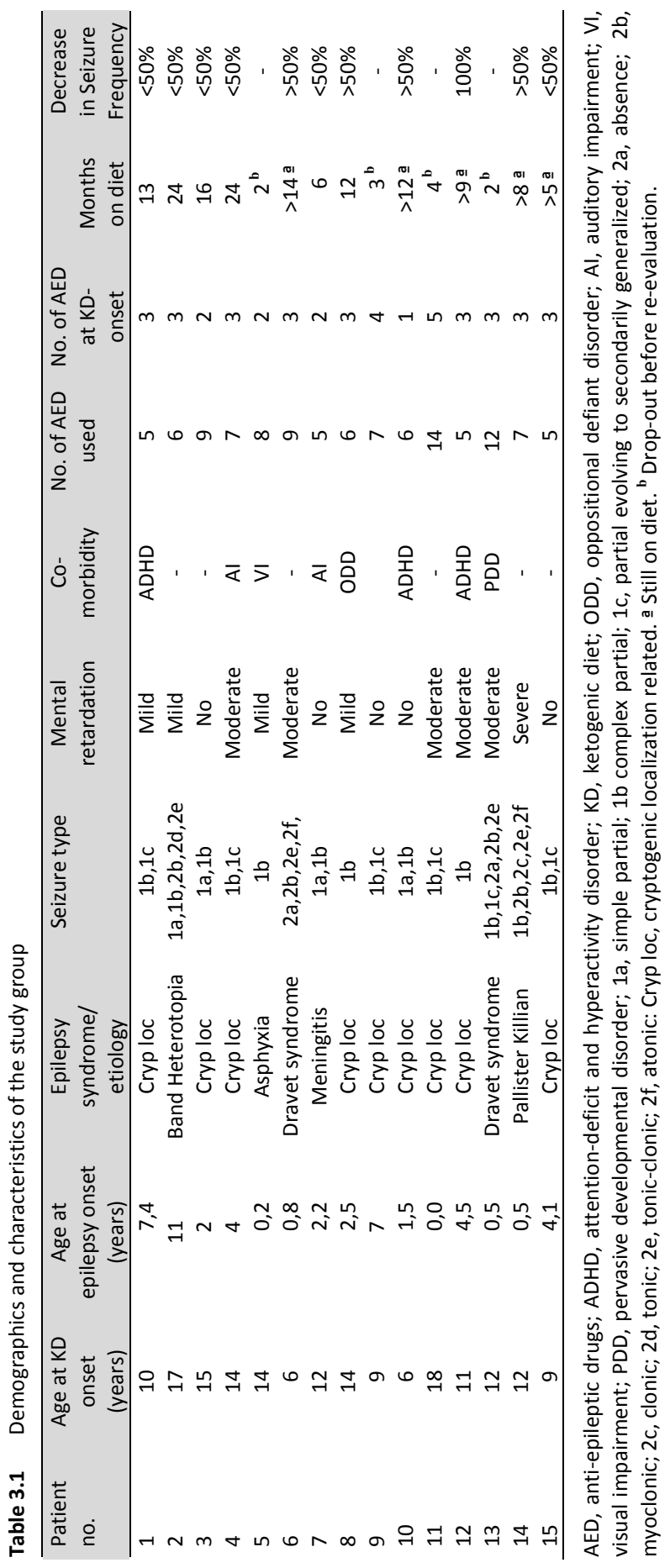




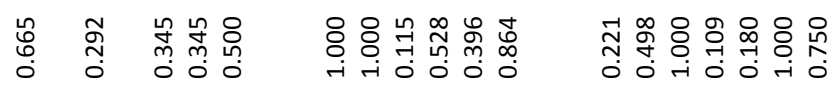

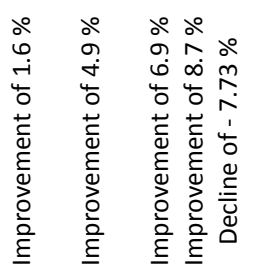

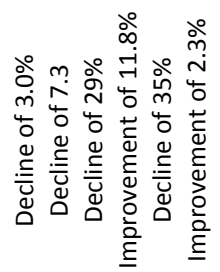

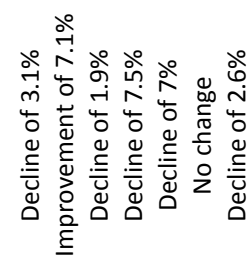

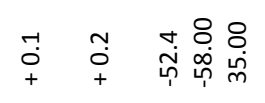

g용유 듐

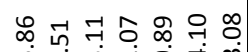

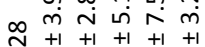

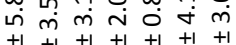

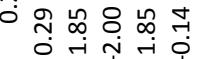

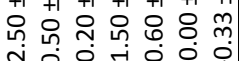

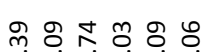

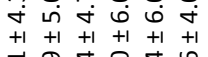

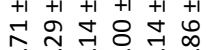

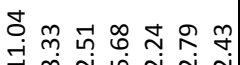

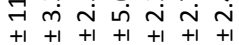

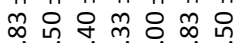

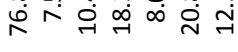

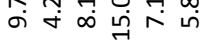

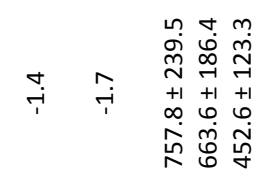

ๆ $\hat{m} \mathcal{F} \hat{m}$

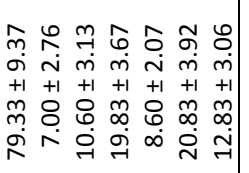

年

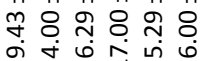

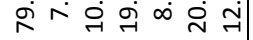




\section{Discussion}

The efficacy of the diet in terms of reduction in seizure frequency in our group (five of 15 patients) was consistent with previous findings (38\%). ${ }^{6}$

Our study is the first to use a comprehensive battery of cognitive tests, administered to children themselves to assess the effects of the KD after 6 months. Because of the unknown effects of KD on cognition and behavior, it was decided to use a global but extended assessment battery. Therefore, in addition to global cognition, assessment includes also specific attention functions, locomotion, mood, behavior, and psychosocial adjustment.

Recently, Nikanorova et al. ${ }^{29}$ reported an increase in IQ in one of five children with continuous spike-waves during sleep (CSWS) after 12 and 24 months. In our study, the score on the PPVT, as a measure of global cognitive level, only showed a small but non-significant change during KD treatment. A possible detrimental effect of the KD on cognitive function described in animal studies was not found in any of our subjects. ${ }^{30}$ In general, the majority of the cognitive tests that we used, assessing global cognitive function, attention, and $\mathrm{VMI}$, improved in every patient assessed. On average, the positive changes were too small to achieve statistical significance; furthermore, we found a slight improvement in reaction times.

The improvement of attention is a well-known effect of the KD. ${ }^{8,9,14,16}$ In individual patients, this was associated with positive changes in behavior, which is in accordance with the previous reports of improved behavior in a subset of children. ${ }^{13,16}$

In contrast, the POMS showed mood changes in the negative direction. Some changes were in the large range (up to $35 \%$ negative change) and would yield statistical significance in a larger sample. If we nonetheless interpret the negative changes, then the pattern showed an impairment of 'energy' (fatigue, less vigor) and more anger and hostility.

In non-epileptic mice, the KD seems to have mood-stabilizing effects. ${ }^{31}$ Previously, ElMallakh and Paskitti ${ }^{32}$ proposed that the KD might also have mood-stabilizing effects in bipolar disorder. We were not able to confirm this hypothesis, probably because our subjects were not suffering from major affective symptoms at baseline.

Overall, we found only a slight decrease in psychosocial adjustment. This is in contrast to previous findings of an improvement in social functioning in children on KD treatment. ${ }^{8}$ A probable factor in this discrepancy is that patients' mean age was significantly higher in our study (12 years compared to 5 years in the study by Pulsifer et al.), possibly resulting in more problems adapting to the diet.

Our study has several shortcomings. Because of the complex and refractory nature of the subjects' epilepsy, we were unable to define a suitable control group. We therefore decided to apply a pretest/posttest design in which each patient is his or her own control. Although we used neuropsychological tests with a low capability of practice effects, we cannot exclude some influence. Nevertheless, a retest period of 6 months is reasonable especially when compared with retest periods of 12 weeks 
used in drug trials. Furthermore, there is evidence from the literature that there is a lack of traditional practice effects in patients with chronic temporal lobe epilepsy. ${ }^{33}$ In addition, only a small number of patients were included. Consequently, most results did not reach the level of statistical significance. However, despite such limitations, we think that our results do indicate interesting trends such as the absence of a negative impact on cognition or social adaptation and a tendency toward an increase in mood problems. The latter effects require adequate management in clinical practice. 


\section{References}

1. Deonna T, Roulet Perez E, Aicardi J. Cognitive and behavioural disorders of epileptic origin in children. London: Mac Keith Press, 2005.

2. Freeman JM, Kossoff EH, Hartman AL. The ketogenic diet: one decade later. Pediatrics 2007;119: 535-43.

3. Henderson CB, Filloux FM, Alder SC, Lyon JL, Caplin DA. Efficacy of the ketogenic diet as a treatment option for epilepsy: meta-analysis. J Child Neurol 2006;21:193-8.

4. Keene DL. A systematic review of the use of the ketogenic diet in childhood epilepsy. Pediatr Neurol 2006;35:1-5.

5. Freeman JM, Vining EP, Pillas DJ, Pyzik PL, Casey JC, Kelly LM. The efficacy of the ketogenic diet-1998: a prospective evaluation of intervention in 150 children. Pediatrics 1998;102:1358-63.

6. Neal EG, Chaffe $\mathrm{H}$, Schwatz RH et al. The ketogenic diet for the treatment of childhood epilepsy: a randomised controlled trial. Lancet Neurol 2008;7:500-6.

7. Farasat S, Kossoff EH, Pillas DJ, Rubenstein JE, Vining EP, Freeman JM. The importance of parental expectations of cognitive improvement for their children with epilepsy prior to starting the ketogenic diet. Epilepsy Behav 2006;8:406-10.

8. Pulsifer MB, Gordon JM, Brandt J, Vining EP, Freeman JM. Effects of ketogenic diet on development and behavior: preliminary report of a prospective study. Dev Med Child Neurol 2001;43:301-6.

9. Hallböök T, Lundgren J, Rosen I. Ketogenic diet improves sleep quality in children with therapyresistant epilepsy. Epilepsia 2007;48:59-65.

10. Weber S, Molgaard C, Taudorf K, Uldall P. Modified Atkins diet to children and adolescents with medical intractable epilepsy. Seizure 2009;18:237-40.

11. Seidel WT, Davis K, Lin M, Mitchell WG, Chen LS. Ketognic diet: seizure outcome and parental reports. Epilepsia 1997;38 (Suppl. 8):196-7.

12. Bergqvist AG, Chee CM, Lutchka LM, Brooks-Kayal AR. Treatment of acquired epileptic aphasia with the ketogenic diet. J Child Neurol 1999;14:696-701.

13. Nigro N, Ventimiglia J, Selcen D, Beierwaltes P. Seizure frequency, behavioral, and performance effects of the ketogenic diet. Ann Neurol 1995;38:549-50.

14. Kossoff EH, Pyzik PL, Mcgrogan JR, Rubenstein JE. The impact of early versus late anticonvulsant reduction after ketogenic diet initiation. Epilepsy Behav 2004;5:499-502.

15. Haas RH, Rice MA, Trauner DA, Merritt TA. Therapeutic effects of a ketogenic diet in Rett syndrome. Am J Med Genet Suppl 1986;1:225-46.

16. Kinsman SL, Vinning EP, Quaskey SA, Mellits D, Freeman JM. Efficacy of the ketogenic diet for intractable seizure disorders: review of 58 cases. Epilepsia 1992;33:1132-6.

17. Mak SC, Chi CS, Wan CJ. Clinical experience of ketogenic diet on children with refractory epilepsy. Acta Paediatr Taiwan 1999;40:97-100.

18. Hurk van den TAM, Louw van der EJTM. Dieetbehandelingsrichtlijn ketogeen dieet voor kinderen met refractaire epilepsie. Utrecht: UMC Utrecht, 2007.

19. Schlichting L. Peabody PictureVocabulary Test-III-NL. Amsterdam: Harcourt Test Publisher, 2005.

20. Alpherts WC, Aldenkamp AP. Computerized neuropsychological assessment of cognitive functioning in children with epilepsy. Epilepsia 1990;31(Suppl. 4):S35-40.

21. Beery KE, Beery NA. The Beery-Buktenica developmental test of visual-motor integration: administration, scoring, and teaching manual. Minneapolis, MN: NCS Pearson, 2004.

22. Scholte EM, Pploeg vd JD. Handleiding ADHD-vragenlijst (AVL). Houten: Bohn Stafleu Van Loghum, 2005.

23. Kraijer DW. AVZ-R: Autisme- en verwante stoornissenschaal-Z-revisie: handleiding, 3rd edn. Lisse: Swets Test Publishers (STP), 1999.

24. Mcnair PM, Lorr M, Droppleman LF. POMS manual: profile of mood states, educational and industrial testing service. San Diego, DA: Educational and Industrial Testing Service, 1992.

25. Stein REK, Jessop DJ. Manual for Personal Adjustment and Role Skills Scale III (PARS III). Bronx, New York: Albert Einstein College of Medicine, 1990.

26. Dunn LM, Dunn LM. Peabody picture vocabulary test, 3rd edn. Circle Pines, MN: American Guidance Service Inc, 1997. 
27. Walker DK, Stein RE, Perrin EC, Jessop DJ. Assessing psychosocial adjustment of children with chronic illnesses: a review of the technical properties of PARS III. J Dev Behav Pediatr 1990;11:116-21.

28. Hendriksen JG, Poysky JT, Schrans DG, Schouten EG, Aldenkamp AP, Vles JS. Psychosocial adjustment in males with Duchenne muscular dystrophy: psychometric properties and clinical utility of a parentreport questionnaire. J Pediatr Psychol 2009;34:69-78.

29. Nikanorova M, Miranda MJ, Atkins M, Sahlholdt L. Ketogenic diet in the treatment of refractory continuous spikes and waves during slow sleep. Epilepsia 2009;50:1127-31.

30. Zhao Q, Stafstrom CE, Fu DD, HU Y, HOLMES GL. Detrimental effects of the ketogenic diet on cognitive function in rats. Pediatr Res 2004;55:498-506.

31. Murphy P, Likhodii S, Nylen K, Burnham WM. The antidepressant properties of the ketogenic diet. Biol Psychiatry 2004;56:981-3.

32. El-Mallakh RS, Paskitti ME. The ketogenic diet may have mood-stabilizing properties. Med Hypotheses 2001;57:724-6.

33. Hermann BP, Seidenberg M, Dow C et al. Cognitive prognosis in chronic temporal lobe epilepsy. Ann Neurol 2006;60:80-7. 


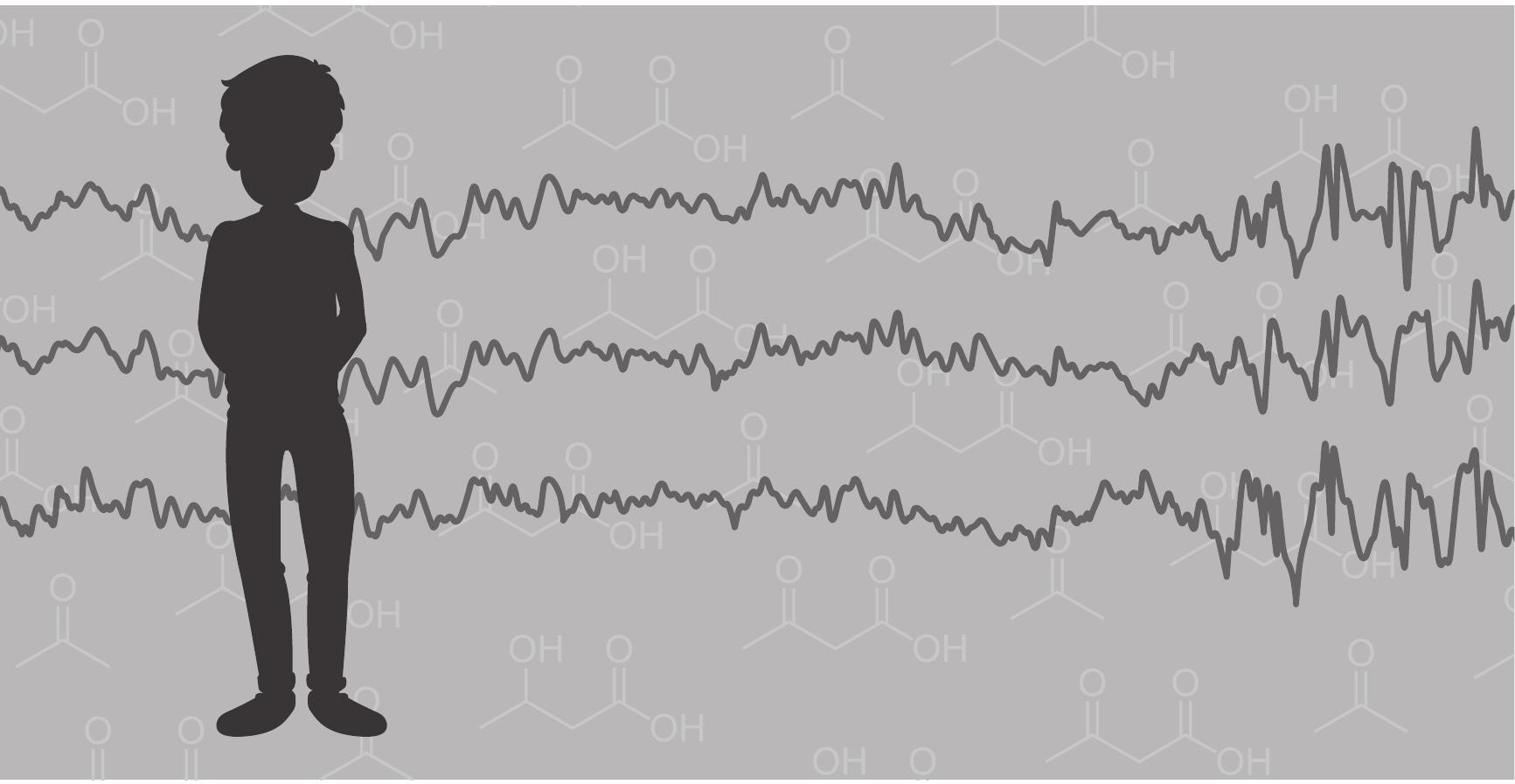




\section{Chapter 4}

\section{The ketogenic diet as a treatment option in adults with chronic refractory epilepsy: efficacy and tolerability in clinical practice}

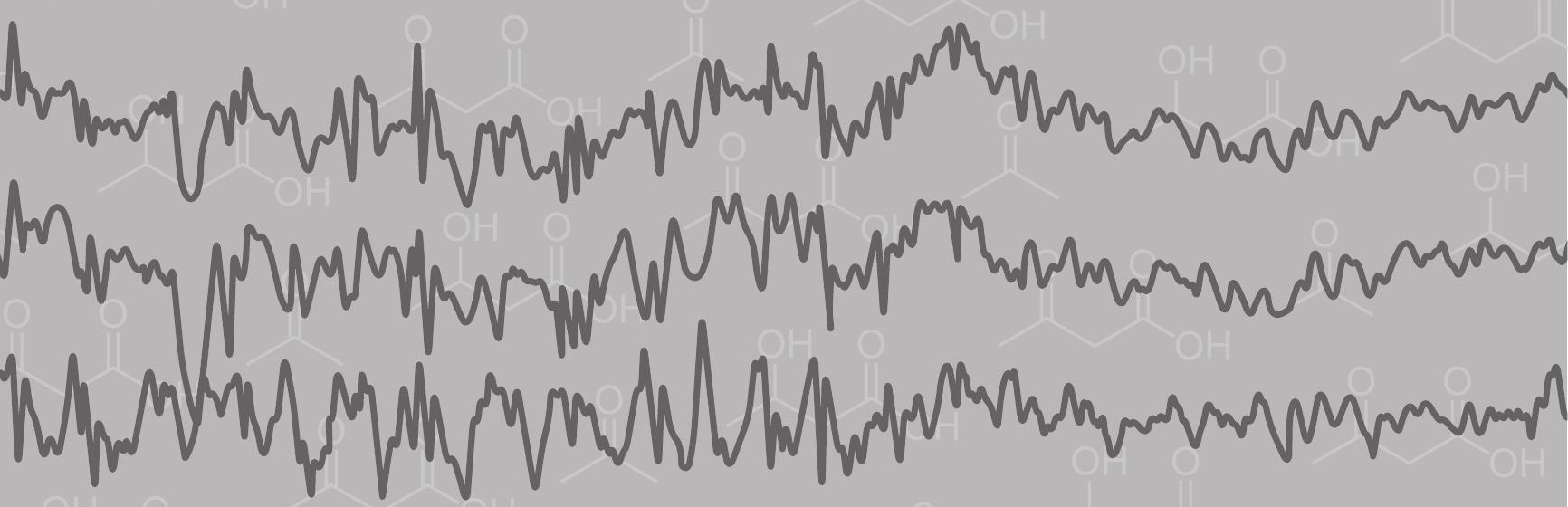

D.A.J.E. Lambrechts, L.H.P. Wielders, A.P. Aldenkamp, F.G.H. Kessels, R.J.A. de Kinderen, H.J.M. Majoie 


\section{Abstract}

The ketogenic diet (KD) is a high-fat, low-protein, low-carbohydrate diet that is used as a treatment for patients with difficult-to-control epilepsy. The present study assesses the efficacy and tolerability of the KD as an add-on therapy in adults with chronic refractory epilepsy. 15 adults were treated with the classical diet or MCT-diet. During a follow-up period of 1 year we assessed seizure frequency, seizure severity, tolerability, cognitive performance, mood and quality of life (QOL). We found a significant reduction in seizures among the patients who followed the diet at least 1 year $(n=5)$. Of these 5 patients, 2 had a reduction between 50 and $90 \%$. Analyzing the study months separately, we found a seizure reduction of $\geq 50 \%$ in $26.6 \%$ of the patients during at least 1 month of treatment. Common side-effects were gastrointestinal disorders, loss of weight and fatigue. There was a considerable, non-significant improvement found in mood and QOL scores.

Improvements were independent of reduction in seizure frequency, indicating that the effects of the KD reach further than seizure control. 


\section{Introduction}

Epilepsy is a common neurologic disease, usually treated with anti-epileptic drugs (AEDs). However, approximately $30 \%$ of patients cannot be adequately controlled by AEDs. Other treatment options, including the ketogenic diet (KD), have therefore been introduced.

Fasting has been practiced as a treatment for epileptic seizures since biblical times. The first description of the ketogenic diet in literature dates from the 1920s. Wilder suggested the ketogenic diet as a treatment for epilepsy, since it would have similar effects as fasting. ${ }^{1}$ The ketogenic diet is a high-fat, low-protein, low-carbohydrate diet that is used as a treatment option for patients with AED-resistant epilepsy. The exact mechanisms underlying the ketogenic diet are unknown. ${ }^{2}$

There are various forms by which the KD is used as a treatment for epilepsy. The classical KD consists of long-chain triglycerides (LCTs), most often applied in a KD-ratio of 4:1 for [fat]:[proteins and carbohydrates]. Another well known form of the KD is the MCT-diet, which consists mainly of medium-chain triglycerides (MCTs). Due to a more efficient oxidation of these medium chain fatty acids, less fat is needed to maintain sufficient ketosis. As a result, this diet is less restrictive in comparison to the classical diet. ${ }^{3}$ A recent randomized trial of Neal et al. ${ }^{4}$ showed the classical diet did not have any advantage over the MCT-diet in terms of efficacy. The efficacy of the KD has been studied almost exclusively in children. During treatment, 3-33\% of children became seizure free, $7-56 \%$ had a seizure reduction of $\geq 90 \%$ and $19-38 \%$ had a reduction of 50-90\%. ${ }^{3,5-9}$ A recent randomized trial of Neal et al. ${ }^{3}$ demonstrated a significant reduction in seizure frequency in the treatment group in comparison to the control group at 3 months. Mean seizure frequency decreased by $38 \%$ in the treatment group and increased by $37 \%$ in the control group.

Despite its success in children, modest evidence is available about the efficacy of the KD in adults with refractory epilepsy. The largest study dates from 1930, when Barborka et al. studied 100 adults who used the classical KD as a monotherapy. $12 \%$ of these patients became seizure free during treatment, $44 \%$ improved and $44 \%$ remained unchanged. ${ }^{10}$ In the more recent studies, $22-55 \%$ of the patients had a seizure reduction of $\geq 50 \%$ at various follow-up time points ranging from 12 weeks to 8 months. ${ }^{11-15}$ Even though seizure control is an important measure of efficacy, it is important to observe the effects of the KD on several psychological functions. In our opinion these aspects are important determinants of the tolerability of the diet. Previous studies showed improved cognition and arousal to correlate better with the retention on the diet than do seizure control or reduction of AEDs. ${ }^{7,12}$ Fatigue is a reported side-effect of the $\mathrm{KD} .^{3,10,16}$ On the other hand, improved attention is mentioned as a positive effect in $13-63 \% .^{3,15,18}$ In addition one can assume that the restrictions that follow KD influence mood and quality of life (QOL) in a negative way, especially in adults. Overall QOL has been investigated in adults with refractory epilepsy, but no significant changes have been found during the KD. ${ }^{13,14}$ 
In the present study we assessed the efficacy and tolerability of the KD as an add-on therapy in adults with chronic refractory epilepsy.

\section{Methods}

This prospective observational clinical study was accomplished at the Epilepsy Center Kempenhaeghe, Heeze, The Netherlands. Patients were included in the period January 2005 until September 2010. The study was approved by the Medical Ethics Committee and all subjects signed informed consent.

\section{Patients}

Patients were referred to Kempenhaeghe by neurologists from hospitals all over the country. Men and women, aged $\geq 18$ years, with refractory epilepsy were recruited for the study. Refractory epilepsy was defined as seizures not adequately controlled by optimal treatment with $\geq 3$ AEDs. All types of seizures and syndromes were accepted and both were classified according to the revised terminology presented by Berg et al. ${ }^{17}$ Patients were not eligible for epilepsy surgery. Exclusion criteria were metabolic diseases (e.g. DM, fatty acid metabolism disease), malignancies, progressive cerebral lesions, degenerative diseases, hyperlipidemia, hypercholesterolemia, long QTsyndrome, myocardial infarction, TIA, CVA, peripheral vascular disease, malnutrition, pregnancy and severe behavioral problems or insufficient motivation, In contrast with other studies treatment with topiramate or acetazolamide combined with a positive family history of kidney stones or metabolic acidosis was an exclusion criterion.

\section{Baseline evaluation}

Patients were evaluated by a team consisting of an epileptologist, an internist, a nurse practitioner and a nutritionist. Patients met separately with each team member before initiation of the diet. The nutritionist calculated sample recipes, according to the patient's needs and preferences.

Blood and urine samples were taken. Blood samples assessed electrolytes, $\mathrm{pH}$, lipid levels, liver function, kidney function, albumin, amylase and hematology. Serum levels of current AEDs were checked. Blood samples were examined to gather further information regarding possible metabolic diseases. Urine samples were checked for specific gravity (S.G.), pH, protein, glucose, ketone bodies, erythrocytes and nitrite. A pregnancy test was performed in all female subjects. An ECG and in some cases an EEG were performed.

Quality of life (QOL) was evaluated at baseline, using the Quality of Life in Epilepsy Inventory (QOLIE-89). ${ }^{19}$ Raven's Progressive Matrices were accomplished to assess 
global cognitive performance. ${ }^{20}$ Profile of Mood States (POMS) was used to assess mood. $^{21}$

\section{Introduction of the diet}

The KD was introduced during a 2-week hospitalization at the Epilepsy Center Kempenhaeghe. Usually the MCT-diet was applied, but if insurance did not refund the fat-emulsions of the MCT-diet or on subjects' request, the classical diet was used. When tube feeding was given, a liquid form of the classical KD was used. Changes were made to the MCT-diet in some patients, producing a mixed form of MCT-diet and the classical diet.

The diet was introduced according to the Dutch guideline for KD. ${ }^{22}$ This guideline does not include a fasting period before introduction. In the classical diet the fat intake was gradually built up in 4-5 days. In the MCT-diet it takes 2-3 weeks to build up the fat to the required ratio: starting with LCT-fat and only $10 \mathrm{~g}$ of MCT-fat to $100 \%$ of the calculated fat amount. Then MCT-fat is slowly increased and LCT-fat is withdrawn.

Vitamin supplements were administered to avoid deficiencies. The start of the diet was defined as the first change made to the daily nutrition of the patient.

\section{Evaluation}

Evaluation took place at 7 weeks after initiation of the KD and every 3 months later. Seizure frequency, seizure severity, adverse effects and compliance were evaluated at each visit. Time of discontinuation of the diet and reasons for discontinuation were recorded.

Daily seizure calendars were completed during a 3-month baseline period and during treatment. Reduction in seizure frequency was calculated by comparing the amount of seizures during every month after initiation of the KD to the mean baseline frequency. Responders were defined as patients with $\geq 50 \%$ reduction in seizure frequency. Seizure severity was assessed using the National Hospital Seizure Severity Scale (NHS3). ${ }^{23}$

Overall benefits of the KD in clinical practice were evaluated using retention rates. Retention rates express the willingness of a patient to continue the diet. It is considered to be a composite of diet efficacy and safety and is therefore an important clinical parameter. ${ }^{24}$

During baseline and during the first 6 months on the diet extra medication was only given if medically necessary. In case of adequate efficacy, the opportunity of reducing AEDs was given after 6 months.

Blood samples were taken from all patients at each visit. Laboratory evaluations included the same examinations as baseline, plus levels of $\beta$-hydroxybutyrate (BHB). Previous studies found BHB to correlate better with seizure reduction than do urinary ketones. ${ }^{25}$ Nevertheless, urine ketone levels were checked every day at similar moments using Ketostix. Levels of ketosis were recorded in the seizure calendar. 
Neuropsychological assessment was carried out at approximately 8 months after initiation of the diet and was similar as the assessment at baseline.

\section{Statistics}

Statistical analysis was performed using SPSS 18.0 for Windows. Data were analyzed using Wilcoxon's signed-rank test for nonparametric data (two-tailed). Significance level was set at $p<0.05$. Retention rates were calculated using Kaplan-Meier survival analysis.

\section{Results}

Fifteen adults were included. Table 4.1 summarizes the patients' demographics, disease characteristics and treatment at baseline. Seven male and eight female subjects with a mean age of 28 years (range 18-41) were included. Monthly seizure frequency at baseline ranged from 4 to 128 seizures/month (mean 27.6) and most patients suffered from complex partial and/or secondary generalized seizures. Most patients were diagnosed with localization-related epilepsies $(n=11)$. A mean of 8.4 (range 5-14) AEDs was used before initiation of the diet, 2 patients were treated with vagus nerve stimulation (VNS). At initiation of the diet a mean of 2.7 AEDs (range $0-6)$ was used.

Eleven patients (74\%) started the MCT-diet, 2 patients (13\%) started the classical diet and 2 patients (13\%) were treated with a combination of both diets. In these 2 patients transition from the MCT to the classical diet was made because of gastrointestinal complaints.

\section{Seizure control}

Four patients (26.6\%) had a seizure reduction of $\geq 50 \%$ during at least 1 month of treatment. At 4 months $(n=9)$ and 6 months $(n=6), 1$ patient had a seizure reduction of $50-90 \%$. At 12 months ( $n=5), 2$ patients had a seizure reduction of 50-90\% (Table 4.2). Figure 4.1 shows that some patients had an increased seizure frequency at 4 and/or 6 months of treatment. Of these patients, 36\% stopped treatment early. In other patients a reduction in seizure frequency was found later. We found no significant reduction of seizure frequency during the treatment period ( $p>0.05$ ), based on an intention-to-treat analysis, using the last observation carried forward method. However, looking only at the patients $(n=5)$ who were still following the diet at 12 months, we found a significant reduction in seizures compared with baseline. Of these patients, 2 had a seizure reduction of $50-90 \%$ and 3 patients had a reduction of less than $50 \%$.

During treatment there were no significant changes in seizure severity, according to the NHS3. 

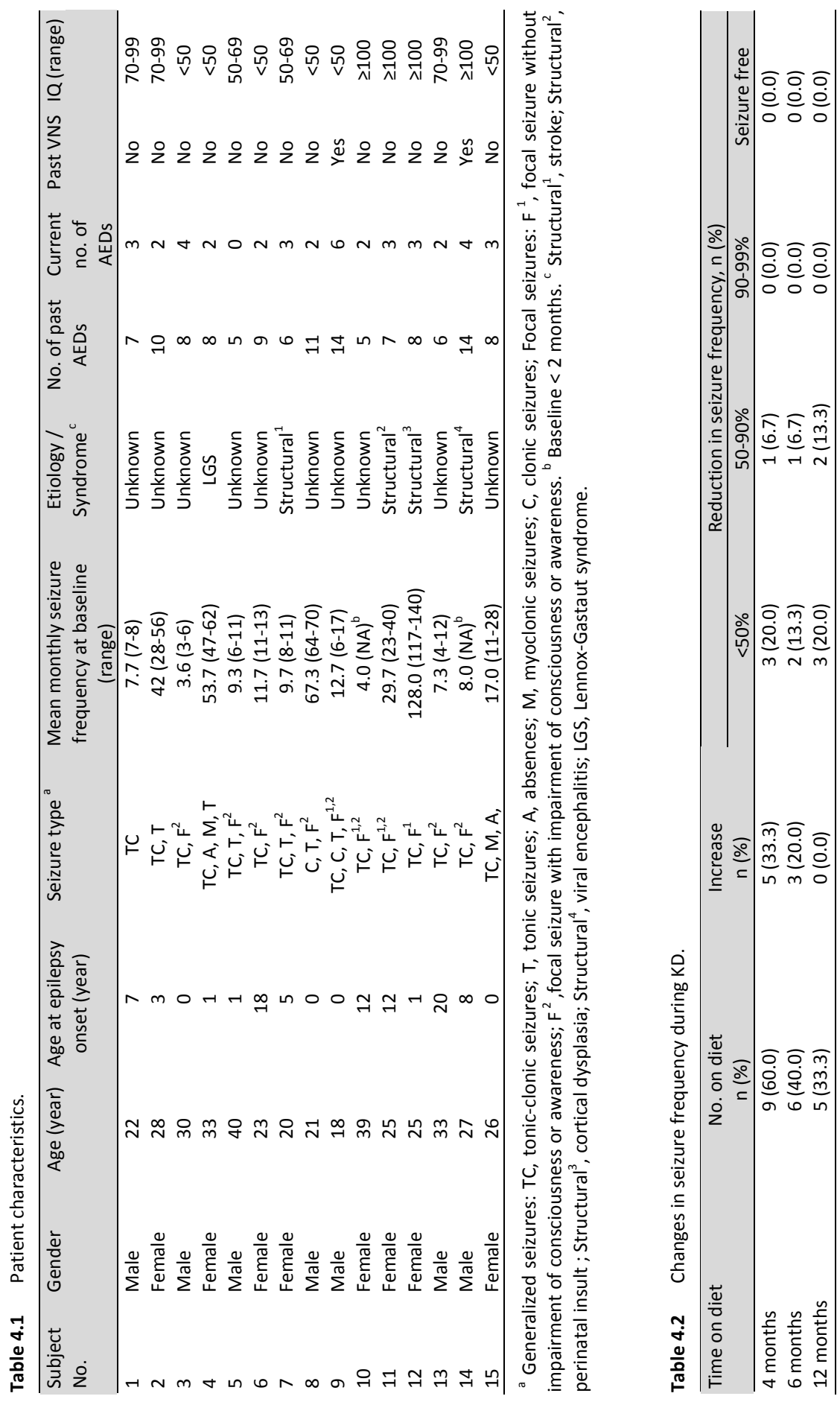


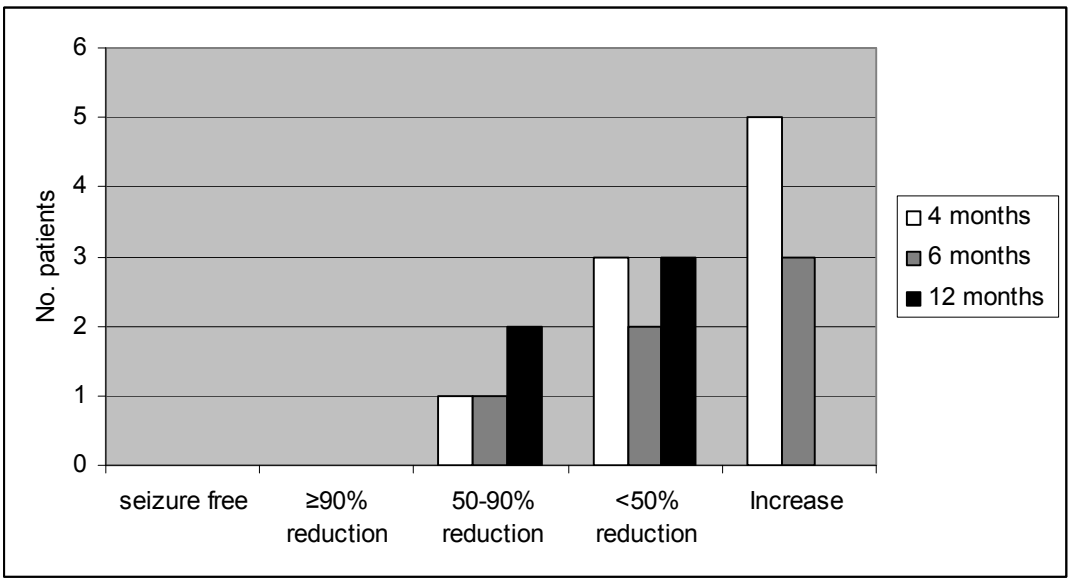

Figure 4.1 Changes in seizure frequency during the KD.

\section{Psychological outcome}

Nine patients (60\%) reported an improved arousal during treatment. There was no significant change in global cognitive performance, according to Raven's Progressive Matrices. With regard to mood we found an improvement of depressed mood of $24 \%$, an improvement of tension and anxiety of $15.7 \%$, a decrease of fatigue of $21.1 \%$ and an improvement of activity of $12.6 \%$. Despite the noticeable results, none of these changes were significant, probably as a result of the small sample size in the study. We found a non-significant increase in QOL from a mean of 60.7 at baseline to 69 at follow-up.

\section{Tolerability}

Table 4.3 shows which side events were reported during the KD. Two out of every 3 patients reported gastrointestinal disorders, including vomiting, diarrhea and constipation. $47 \%$ of patients reported extreme fatigue during their treatment and $60 \%$ lost $\geq 2 \mathrm{~kg}$ of weight. Two patients developed lipid disorders. No female patient reported menstrual irregularities. One patient developed nephrolithiasis after $>1$ year on treatment and had to discontinue for this reason.

Figure 4.2 shows the retention rate for this population. Ten patients discontinued their diet within 1 year after initiation, at a mean of 0.35 years (range $0.17-0.75$ years). The other patients $(n=5)$ continued their diet for over a year (mean duration=1.6 years), of which 2 patients are still ongoing. As can be seen in Table 4.3, most patients stopped the diet because of inefficacy. 


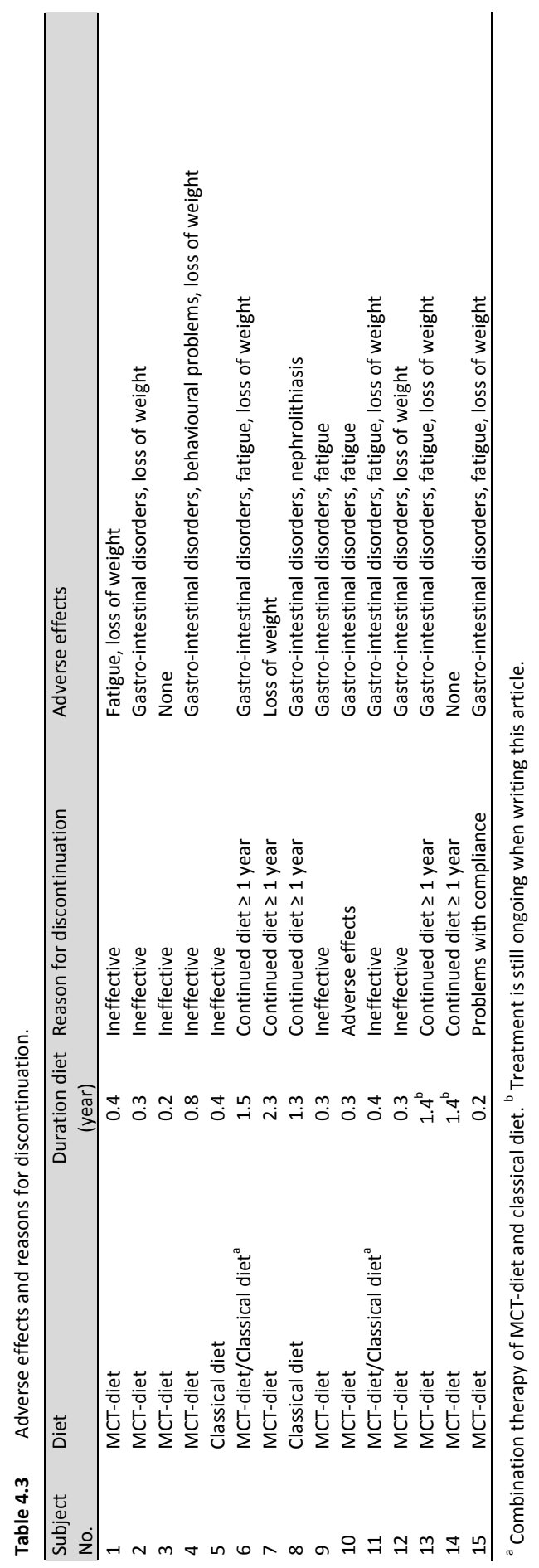




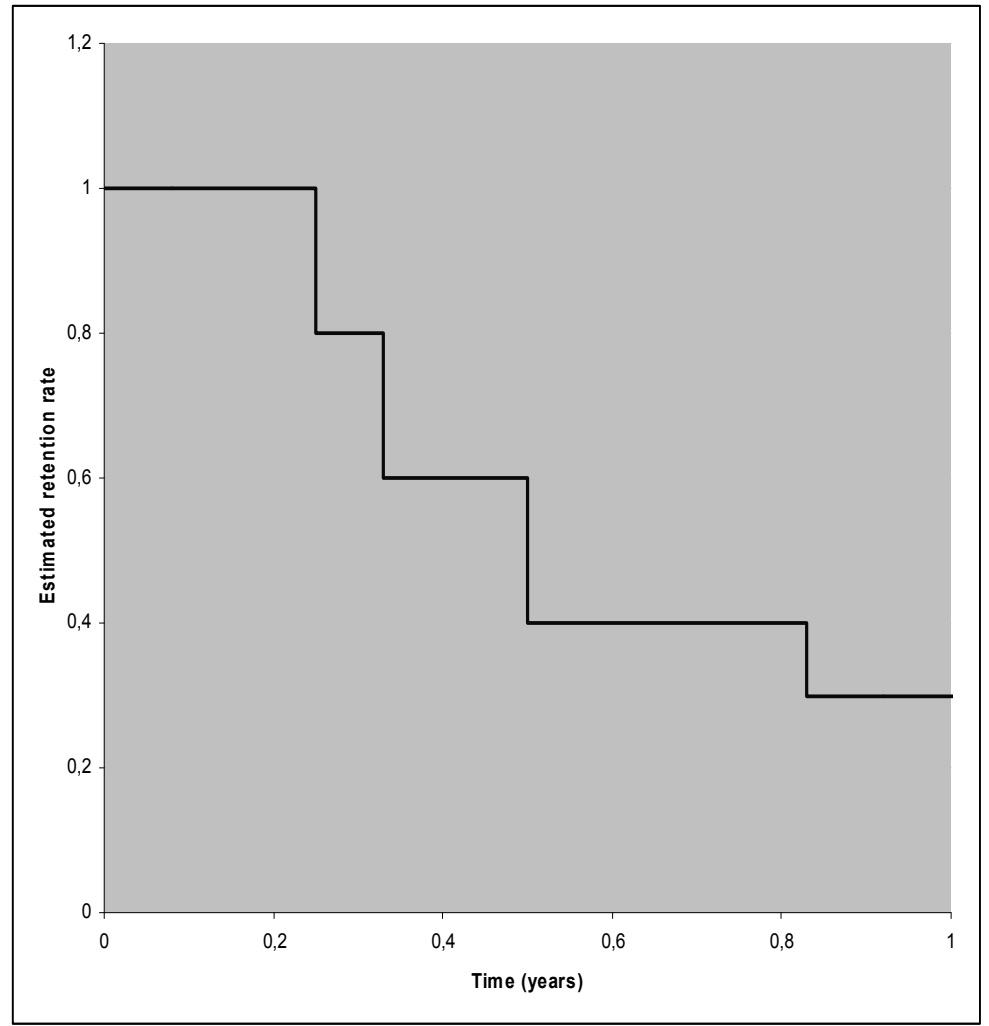

Figure 4.2 Retention rate for patients on KD.

\section{Ketosis}

We used BHB in serum as an indicator of the level of ketosis in patients on the diet. Mean $\mathrm{BHB}$ at 4,6 and 12 months was $0.8,0.6$ and $1.8 \mathrm{mmol} / \mathrm{l}$ respectively. Two patients showed an elevated BHB ( $\geq 2 \mathrm{mmol} / \mathrm{l})$ during treatment, but none of them showed an elevated $\mathrm{BHB}$ during the whole treatment.

Urinary ketosis was elevated ( $\geq 8 \mathrm{mmol} / \mathrm{l}$ ) in $42 \%, 50 \%$ and $40 \%$ of the patients at 4,6 and 12 months respectively.

\section{AEDs}

Patients used a mean of 2.73 AEDs at initiation of the KD. In 3 patients, AEDs were changed during baseline. In 3 patients, changes in AEDs were made during the first 6 months following the initiation of the diet. Changes in AEDs concerned dose reductions or termination of an AED. 
In case of adequate efficacy of the KD, the opportunity of reducing AEDs was given after 6 months. At 1 year after initiation of the diet, patients used a mean of 2.2 (range 0-5) AEDs. Patients who stopped the diet early used a mean of 2.8 AEDs (range 0-6) at discontinuation of the diet.

\section{Discussion}

The main purpose of this study was to assess the efficacy and tolerability of the KD as an add-on therapy in adults with chronic refractory epilepsy. Analyzing the study months separately, we found a seizure reduction of $\geq 50 \%$ in $26.6 \%$ of the patients during at least 1 month of treatment. This is in line with previously reported results. ${ }^{11-15}$ Moreover, we found a substantial improvement in mood and an increase of QOL in adults on the KD.

The advantage of this study is that it has a long follow-up period of 1 year. Moreover, most previous studies evaluated the efficacy of the KD at one defined moment, whereas this study evaluates the changes in seizure frequency at 4, 6 and 12 months. Overall tolerability of the diet is evaluated using retention rates. Furthermore, this study provides a good insight into the effects of the KD on psychological function. Some previous studies mentioned the effects of the KD on QOL or mood in adults. ${ }^{13,14}$ However, to our knowledge this is the first study which assesses the effects of the KD on overall psychological functioning (cognition, mood and QOL) in adults with chronic refractory epilepsy.

Limitations of the study include its small sample size, uncontrolled selection of patients and the nonrandomized design. In addition, AEDs were changed in 3 patients during baseline and in 3 patients during the first 6 months after initiation of the KD. Even though we were aiming to preserve the baseline medication, it was not always possible due to the complexity of the cases in this study.

$26.6 \%$ of the patients had a seizure reduction of $\geq 50 \%$ during at least 1 month of their treatment. However, when assessed at specific moments during the treatment, the effect of the KD on seizure frequency was considerably less promising. At 4 and 6 months, only 1 patient (6.7\%) had a seizure reduction of $\geq 50 \%$. Taking only the patients $(n=5)$ who were still following the diet at 12 months into account, we found a significant reduction in seizures compared with baseline. Of these patients, 2 had a seizure reduction of $50-90 \%$ and 3 patients had a reduction of less than $50 \%$.

In general the KD and its variants may lead to similar reductions in seizure frequency in adolescents and adults as seen in children, although studies are limited and of poor quality. Few studies have reported use of the KD exclusively in adults. In three studies the classical KD was used, in another three studies the modified Atkins diet was used. It is unlikely that our patients suffer from more severe refractory epilepsy. On average they were treated with 8.4 different AEDs before initiation of the diet. There is at least some evidence from the literature that people with symptomatic generalized epilepsy 
may respond better to dietary treatment than those with idiopathic generalized epilepsy and partial epilepsy. Also people with multiple seizure types might respond better. However, most of our patients were classified with partial epilepsy with only a few different seizure types. Payne et al. indicate that noncompliance may be higher in adolescents and adults than in children. ${ }^{26}$

There were 5 patients (33\%) showing an increased seizure frequency during the KD. This has been reported previously. The study of Barborka et al. ${ }^{10}$ showed an increase in seizure frequency in $6 \%$ of the patients. The same was true for $8 \%$ of the patients in the study of Klein et al. ${ }^{14}$ Nevertheless, our study found an increase in seizure frequency in $33 \%$ of patients, which is considerably higher than mentioned in these previous studies. Several contributing factors were found to explain this difference. Two patients, both of whom were mentally retarded, had poor compliance. One of these 2 patients showed an increased seizure frequency prior to introduction of the diet. It is imaginable that the increased seizure frequency during treatment reflects the natural course of his disease. One patient reported extreme abdominal pains and diarrhea, due to which the diet could not be fully introduced. One patient did not report auras during baseline and started to report these seizures some weeks after initiation of the diet. In 1 patient no plausible reason for the increased seizure frequency was found.

A major problem in our study was the low serum BHB of $<2 \mathrm{mmol} / \mathrm{l}$ in most of the patients.

At 3 and 6 months, van Delft et al. found a significant correlation for BHB to seizure reduction. $^{25}$

Nevertheless our current understanding of the importance of ketosis to seizure control is inadequate. Ketone measurements are useful in assessing patient dietary compliance but their usefulness in predicting seizure outcome is unclear. ${ }^{27}$ We need to consider the possibility that compliance was not always optimal.

The overall benefits of the KD in clinical practice were evaluated using retention rates. $A$ retention rate expresses the willingness of patients to continue their diet and is considered to be a composite of diet efficacy and tolerability (including psychological benefits and disadvantages). Therefore it is considered as a clinically relevant parameter. Patients discontinued their diet at a mean of 0.8 years, which is in line with the results of a meta-analysis by Henderson et al. ${ }^{28}$ In the present study $33.3 \%$ of the patients continued the diet for at least 1 year. This is below the estimated retention rate at 1 year for new anti-epileptic drugs, which is approximately $52-75 \%{ }^{24}$ This is probably a result of the severe restrictions and frequently reported side-events during the KD.

Major reasons for discontinuation of the diet in previous studies were either ineffectiveness or problems with compliance. $6,7,28,29$

In the present study, nearly all patients who discontinued the diet did so because of inefficacy. Nevertheless, it is obvious that inefficacy, adverse events and problems with compliance are all interconnected. Patients with a substantial reduction in 
seizure frequency are more likely to accept some adverse events and stick to the diet, in comparison to patients in which the diet has no effect.

In the present study, 9 patients (60\%) reported an improved arousal during treatment. This compares well with previous studies, in which $13-68 \%$ of the patients reported an improvement. ${ }^{3,15,18}$ To examine psychological functions in a more objective way, we performed psychological assessments during follow-up. According to the Profile of Mood States, there was a non-significant improvement of mood. Scores of depression, tension, fatigue and activity improved substantially on the KD. Although seizures were not adequately controlled in many patients, mood improved. This could indicate that the effects of the KD reach further than seizure control alone.

As mentioned in the introduction, we expected a decreased quality of life during the $K D$, due to the restrictions and frequently reported side-effects during treatment. Regardless of our hypothesis, QOL showed a non-significant increase. This is presumably due to an improved arousal and improved mood.

Previous studies showed improved arousal and cognition to correlate better with the retention on the diet than do seizure control or reduction of AEDs. ${ }^{8,13}$ A plausible explanation for this is an increased $\mathrm{QOL}$ and improved psychological function during the KD. 


\section{References}

1. Levy R, Cooper P. Ketogenic diet for epilepsy. Cochrane Database Syst Rev 2003(3):CD001903.

2. Freeman J, Veggiotti P, Lanzi G, Tagliabue A, Perucca E. The ketogenic diet: from molecular mechanisms to clinical effects. Epilepsy Res 2006;68:145-80.

3. Neal EG, Chaffe H, Schwartz RH, et al. The ketogenic diet for the treatment of childhood epilepsy: a randomised controlled trial. Lancet Neurol 2008;7:500-6.

4. Neal EG, Chaffe $\mathrm{H}$, Schwartz $\mathrm{RH}$, et al. A randomized trial of classical and mediumchain triglyceride ketogenic diets in the treatment of childhood epilepsy. Epilepsia 2009;50:1109-17.

5. Hemingway C, Freeman JM, Pillas DJ, Pyzik PL. The ketogenic diet: a 3- to 6-year follow-up of 150 children enrolled prospectively. Pediatrics 2001;108:898-905.

6. Freeman JM, Vining EP, Pillas DJ, Pyzik PL, Casey JC, Kelly LM. The efficacy of the ketogenic diet-1998: a prospective evaluation of intervention in 150 children. Pediatrics 1998;102:1358-63.

7. Farasat S, Kossoff EH, Pillas DJ, Rubenstein JE, Vining EP, Freeman JM. The importance of parental expectations of cognitive improvement for their children with epilepsy prior to starting the ketogenic diet. Epilepsy Behav 2006;8:406-10.

8. Lefevre F, Aronson N. Ketogenic diet for the treatment of refractory epilepsy in children: a systematic review of efficacy. Pediatrics 2000;105:E46.

9. Keene DL. A systematic review of the use of the ketogenic diet in childhood epilepsy. Pediatr Neurol 2006;35:1-5.

10. Barborka CJ. Epilepsy in adults. Arch Neurol Psychiatry 1930;23:904-14.

11. Nei SM, Liporace J, Sirven K. Ketogenic diet in adults: response by epilepsy type. Epilepsia 2003;44(Suppl. 9):282.

12. Sirven J, Whedon B, Caplan D, et al. The ketogenic diet for intractable epilepsy in adults: preliminary results. Epilepsia 1999;40:1721-6.

13. Mosek A, Natour H, Neufeld MY, Shiff $Y$, Vaisman N. Ketogenic diet treatment in adults with refractory epilepsy: a prospective pilot study. Seizure 2009;18:30-3.

14. Klein P, Janousek J, Barber A, Weissberger R. Ketogenic diet treatment in adults with refractory epilepsy. Epilepsy Behav 2010;19:575-9.

15. Coppola G, Veggiotti P, Cusmai R, et al. The ketogenic diet in children, adolescents and young adults with refractory epilepsy: an Italian multicentric experience. Epilepsy Res 2002;48:221-7.

16. Lee PR, Kossoff EH. Dietary treatments for epilepsy: management guidelines for the general practitioner. Epilepsy Behav 2011;21:115-21.

17. Berg AT, Berkovic SF, Brodie MJ, et al. Revised terminology and concepts for organization of seizures and epilepsies: report of the ILAE Commission on Classification and Terminology, 2005-2009. Epilepsia 2010;51:676-85.

18. Nordli Jr DR, Kuroda MM, Carroll J, et al. Experience with the ketogenic diet in infants. Pediatrics 2001;108:129-33.

19. Devinsky O, Vickrey BG, Cramer J, et al. Development of the quality of life in epilepsy inventory. Epilepsia 1995;36:1089-104.

20. Raven J, Raven JC, Court JH. Manual for Raven's Progressive Matrices and Vocabulary Scales. Section 1: General Overview. San Antonio; 2003.

21. McNair DM, Lorr M, Droppleman LF. Manual for the Profile of Mood States. San Diego; 1971.

22. van den Hurk ThAM, van der Louw EJTM. Dieetbehandelingsrichtlijn ketogeen dieet voor kinderen (0-18 jaar) met refractaire epilepsie. Evidence-based handleiding voor een multidisciplinaire behandeling. Utrecht: UMC Utrecht: 2007.

23. O'Donoghue MF, Duncan JS, Sander JW. The National Hospital Seizure Severity Scale: a further development of the Chalfont Seizure Severity Scale. Epilepsia 1996;37:563-71.

24. Bootsma HP, Ricker $L$, Hekster YA, et al. The impact of side effects on longterm retention in three new antiepileptic drugs. Seizure 2009;18:327-31.

25. van Delft R, Lambrechts D, Verschuure P, Hulsman J, Majoie M. Blood betahydroxybutyrate correlates better with seizure reduction due to ketogenic diet than do ketones in the urine. Seizure 2010;19: 36-9. 
26. Payne NE, Cross JH, Sander JW, Sisodiya SM. The ketogenic and related diets in adolescents and adults - a review. Epilepsia 2011;52:1941-8.

27. Stafstrom CE, Rho JM. Epilepsy and the ketogenic diet. Totowa, New Jersey: Humana Press; 2004: 133.

28. Henderson CB, Filloux FM, Alder SC, Lyon JL, Caplin DA. Efficacy of the ketogenic diet as a treatment option for epilepsy: meta-analysis. J Child Neurol 2006;21:193-8.

29. Pulsifer MB, Gordon JM, Brandt J, Vining EP, Freeman JM. Effects of ketogenic diet on development and behavior: preliminary report of a prospective study. Dev Med Child Neurol 2001;43:301-6. 


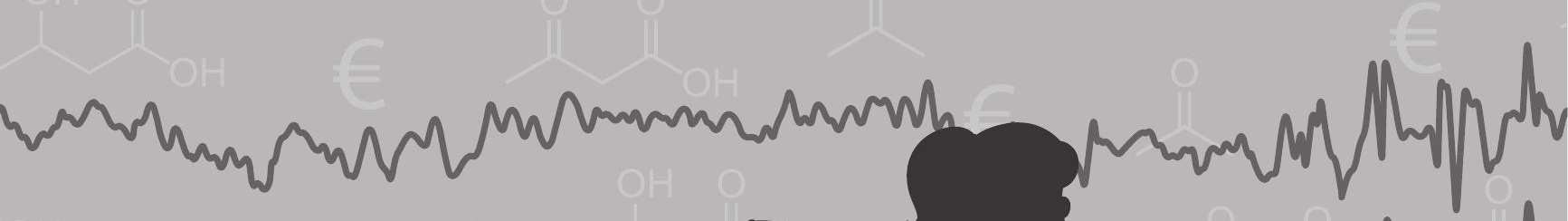

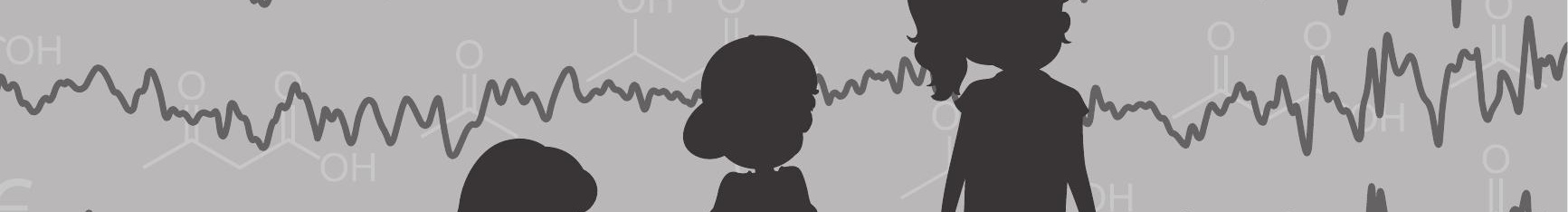




\section{Chapter 5}

Research into the (Cost-)effectiveness of the ketogenic diet among children and adolescents with intractable epilepsy: design of a randomized controlled trial

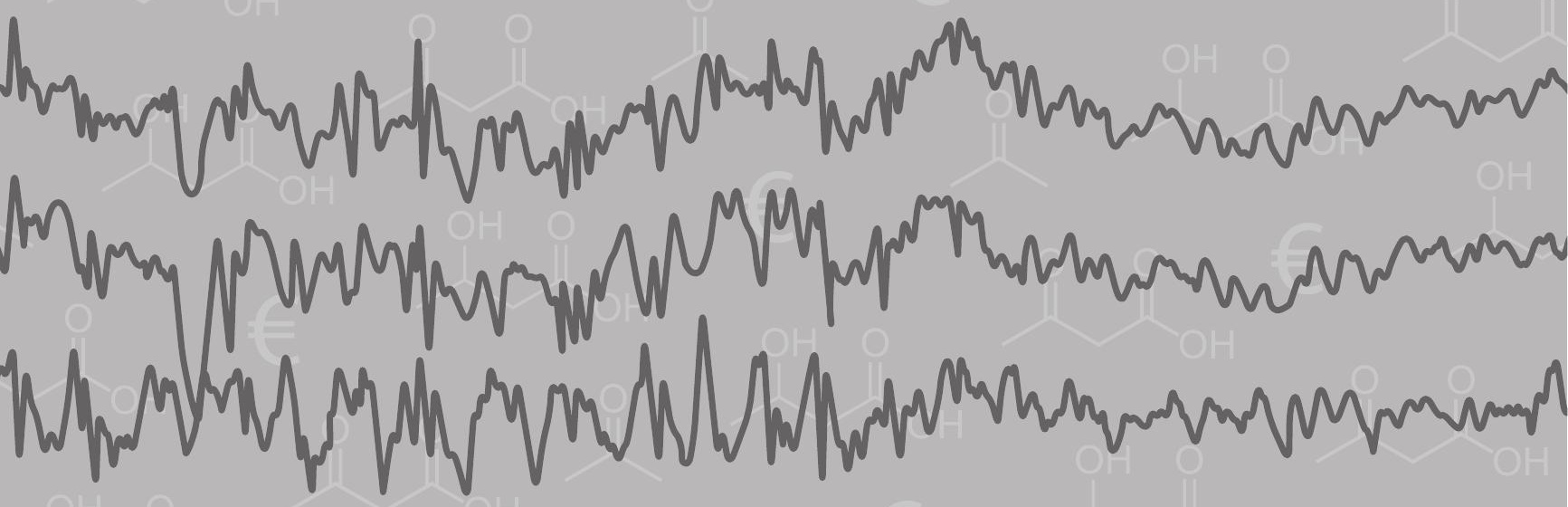

R.J.A. de Kinderen, D.A.J.E. Lambrechts, D. Postulart, A.G.H. Kessels, J.G.M. Hendriksen, A.P. Aldenkamp, S.M.A.A. Evers, H.J.M. Majoie 


\section{Abstract}

\section{Background}

Epilepsy is a neurological disorder, characterized by recurrent unprovoked seizures which have a high impact on the individual as well as on society as a whole. In addition to the economic burden, epilepsy imposes a substantial burden on the patients and their surroundings. Patients with uncontrolled epilepsy depend heavily on informal care and on health care professionals. About $30 \%$ of patients suffer from drug-resistant epilepsy. The ketogenic diet can be a treatment of last resort, especially for children. The beneficial effect of the ketogenic diet has been proven, but information is lacking about its cost-effectiveness. In the current study we will evaluate the (cost-)effectiveness of the ketogenic diet in children and adolescents with intractable epilepsy.

\section{Methods/Design}

In a RCT we will compare the ketogenic diet with usual care. Embedded in this RCT will be a trial-based and model-based economic evaluation, looking from a societal perspective at the cost-effectiveness and cost-utility of the ketogenic diet versus usual care. Fifty children and adolescents (aged 1-18) with intractable epilepsy will be screened for eligibility before randomization into the intervention or the usual care group. The primary outcome measure is the proportion of children with a $50 \%$ or more reduction in seizure frequency. Secondary outcomes include seizure severity, side-effects/complaints, neurocognitive, socio-emotional functioning, and quality of life. Costs and productivity losses will be assessed continuously by a prospective diary and a retrospective questionnaire. Measurements will take place during consults at baseline, at 6 weeks and at 4 months after the baseline period, and 3, 6, 9 and 12 months follow-up after the 4 months consult.

\section{Discussion}

The proposed research project will be the first study to provide data about the costeffectiveness of the ketogenic diet for children and adolescents with intractable epilepsy, in comparison with usual care. It is anticipated that positive results in (cost-)effectiveness of the proposed intervention will contribute to the improvement of treatment for epilepsy in children and adolescents and will lead to a smaller burden to society. 


\section{Introduction}

Epilepsy is a brain disorder characterized predominantly by recurrent and unpredictable interruptions of normal brain function. Seizures are transient occurrences of signs and/or symptoms due to such abnormal excessive or synchronous neuronal activities in the brain. ${ }^{1}$ Different parts of the brain can be the site of such discharges. The clinical manifestations of seizures will therefore vary and depend on where in the brain the disturbance first starts and how far it spreads. Transient symptoms can occur, such as loss of awareness or consciousness and disturbances of movement, sensation, mood or mental function. ${ }^{1}$ Furthermore, epilepsy is a major cost-intensive and worldwide health problem. ${ }^{2}$

In 2000, the aggregate burden due to epilepsy was $0.5 \%$ of the total burden of diseases in the world. ${ }^{3}$ In Europe, about 3.4 million people suffer from epilepsy. ${ }^{4}$ The annual total costs of epilepsy in Europe are $€ 15.5$ billion. ${ }^{5}$ In the Netherlands, direct medical costs were $€ 221$ million in $2005 .^{6}$ In addition to the economic burden, epilepsy imposes a substantial burden on the patients themselves and their surroundings.

In the Netherlands, the point prevalence of epilepsy in children and adolescents between $0-18$ years is 4.77 boys, and 4.55 girls per 1,000 persons. The yearly incidence of epilepsy in the Netherlands for children and adolescents between $0-18$ years is 0.58 per 1,000 boys and 0.53 per 1,000 girls. ${ }^{7}$ Although epilepsy is treatable with anti-epileptic drugs in the majority of cases, about $30 \%$ of patients suffer from drug-resistant epilepsy. ${ }^{8}$ Patients with uncontrolled epilepsy heavily depend on informal care (family and friends) and on health care professionals (neurologists, social workers, psychologists etc.). Complications due to intractable epilepsy result in frequent hospitalizations and many of these patients are institutionalized. Patients with drug-resistant epilepsy can potentially benefit from a ketogenic diet. The ketogenic diet is a high-fat, low carbohydrate, normocaloric diet that mimics the metabolic state of fasting. During a prolonged fast, body energy requirements are met by lipolysis and ß-oxidation of fatty acids rather than by the breakdown of carbohydrates. The ketogenic diet maintains an anabolic nutritional state in a metabolic situation of fasting. Ketone bodies may produce an anticonvulsant effect, presumably due to changes in cerebral energy metabolism, in cell properties decreasing excitability, in neurotransmitter function, in circulating factors acting as neuromodulators and in the brain's extracellular milieu. ${ }^{9}$ The ketogenic diet is generally used for a period of up to 3 years. Seizure control benefits are typically seen within 1-3 months of starting the diet. The international study group reports that the diet should be utilized for at least $31 / 2$ months before deciding to discontinue it. ${ }^{10}$ The diet can be discontinued earlier if seizures worsen beyond expectations or if adverse effects cannot be corrected. ${ }^{11}$ Medications are tapered once the efficacy of the diet has been established (usually within 3-6 months of diet initiation). The diet is gradually tapered off by lowering its fat content and increasing the carbohydrate and 
protein portion of the diet until ketosis is eliminated. Tapering starts after 2 years of treatment, or earlier in case of intolerable side-effects, or in case of ineffectiveness. Currently the scientific and clinical attention paid to the role of the ketogenic diet is negligible. This means that a ketogenic diet is often overlooked and underutilized as a treatment option for children with intractable epilepsy. An important reason for this is that relatively few children and their parents can comply with the stringent diet. Therefore, physicians are often reluctant to initiate the diet. To offer more children the opportunity to benefit from a ketogenic diet, we suggest that the diet should be initiated and monitored under strictly controlled circumstances in order to maximize compliance.

The beneficial effect of a ketogenic diet has been studied in multiple observational studies, ${ }^{12-20}$ reviews ${ }^{21-24}$ and in one randomized controlled trial. ${ }^{25}$ Nevertheless, there is a lack of information about the cost-effectiveness of the ketogenic diet, and consequently health authorities have not been convinced of its usefulness.

The aims of our prospective study are to provide more evidence about the effectiveness and to investigate the cost-effectiveness of treatment with a ketogenic diet KD in comparison with usual care in children and adolescents with drug-resistant epilepsy who are not eligible for epilepsy surgery.

\section{Methods/Design}

The design and methods of a randomized controlled trial (RCT) evaluating the (cost-)effectiveness of a ketogenic diet in comparison with usual care in children and adolescents with intractable epilepsy are described in this paper. This study has been approved by the ethics committee of the Academic Medical Center Utrecht, the Netherlands.

\section{Research question}

We defined the following research questions:

\section{Effect evaluation}

What are the effects of the ketogenic diet in comparison with usual care with respect to changes in seizure frequency and seizure severity, side-effects/complaints, neurocognitive, socio-emotional functioning, and quality of life?

\section{Economic Evaluation}

The economic evaluation will consist of a trial-based and a model-based economic evaluation study. 
Ila) Trial-based economic evaluation

From a social perspective, is the ketogenic diet, in comparison with usual care, preferable in terms of costs, effects and utilities?

What is the incremental cost-effectiveness ratio (ICER) of the ketogenic diet in comparison with usual care?

IIb) Model-based economic evaluation

In comparison with usual care, is the ketogenic diet preferable in terms of costs and utilities during the remaining life expectancy of the study population?

\section{Design}

The proposed study is a prospective RCT. The subjects will be randomized to either the ketogenic diet or to usual care. The trial flow of the proposed subject enrolment and randomization procedures are shown graphically in Figure 5.1. Embedded in this RCT will be a short-term trial-based economic evaluation and a long-term modelling study to investigate the long-term cost-effectiveness and cost-utility of the ketogenic diet versus usual care.

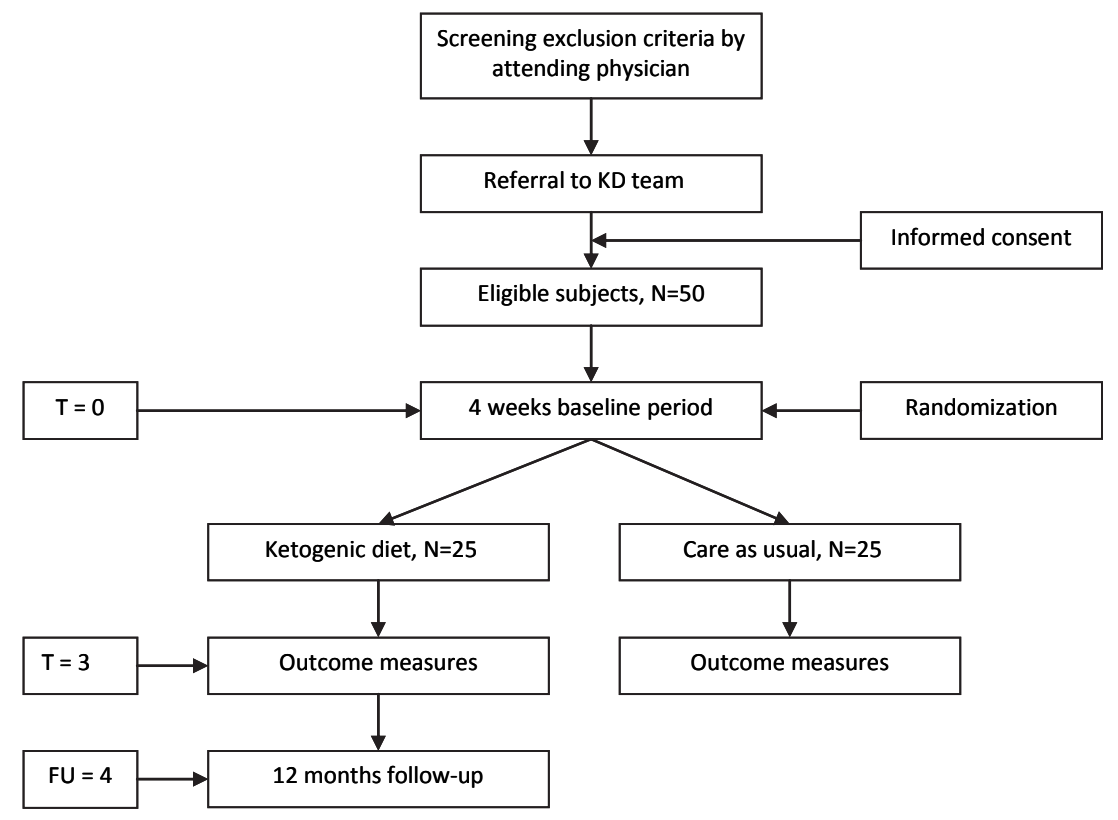

Figure 5.1 Flowchart of the study. 


\section{Participants}

The patient population consists of children and adolescents with drug-resistant epilepsy. Patients are eligible to participate if they meet the following criteria: age between 1 and 18 years old; having uncontrolled seizures; not eligible for epilepsy surgery; no fatty acid oxidation disorders and related diseases; no diabetes and hyperinsulinism; no prolonged QT-time syndrome; no hypercholesterolemia or hypertriglyceridemia; no severe liver, kidney or pancreas diseases; no renal tubular acidosis; no severe behavioral disorder; no malnutrition; no treatment with topiramate or acetazolamide and no positive family history or other risk factors for kidney stones or acidosis.

\section{Recruitment}

Potential participants are screened for exclusion criteria by their own attending pediatrician, neurologist or pediatric neurologist. Children who are eligible to be treated with a ketogenic diet are subsequently referred to the multidisciplinary ketogenic diet team at the epilepsy center Kempenhaeghe in the Netherlands. Eligible children and their parents will first receive information by telephone before getting an invitation for an information visit. During the information visit eligibility is checked by the multidisciplinary team. If they are interested, parents, adolescents and/or children can find information about the study on the website http://www.ketogeenmenu.nl. Parents and potential candidates who are considering participating in the study will have at least one week to decide, before signing informed consent.

\section{Sample size}

A $50 \%$ reduction in seizure frequency is considered clinically relevant and is defined as success. Assuming a minimum detectable difference in success rate of $35 \%$ between the ketogenic diet group and usual care, and assuming that alpha $=5 \%$ and power $=80 \%$, we need 22 children for each group. Taking dropouts into account, we need 50 children in total. A drop-out is defined as a child who drops out of the study before the first consultation with the neurologist; this is scheduled 6 weeks after initiating the KD or 6 weeks after randomization to usual care.

\section{Randomization and procedure}

Randomization will occur halfway through the 4 week baseline period. We will use a software package (ALEA) to support the online patient registration and randomization which will be based on the minimization method. Patients will be stratified according to age (1-6 years, 7-12 years, 13-18 years), having a PEG/ tube or not, and whether the child is living at a residential center or attends the epilepsy center as an outpatient 
while she/he lives at home. In view of the nature of the treatments, blinding of the patients and researchers is not possible.

\section{Intervention}

\section{Ketogenic diet}

Patients assigned to the ketogenic diet group will be hospitalized in the tertiary epilepsy center for one week. During this week the ketogenic diet is introduced by a dietician. The anti-epileptic drugs the children and adolescents use at the time of inclusion in the study will be continued without changes during the study (except when medically indicated). The initial calorie prescription for the ketogenic diet is based on an average between the pre-diet intake and the recommendations for energy requirements, taking into account current and previous weight and height, recommended caloric requirements and levels of physical activity. The dietician will decide together with the parents whether the classical diet or the medium-chain triglyceride (MCT) diet is introduced. Sometimes the MCT diet is not possible and the classical ketogenic diet (3:1 or 4:1 ratio) is advised. Children with a PEG/tube feed are also treated with the ketogenic diet. The diet is then adjusted to a fluid version. It is possible to decrease the titration of anti-epileptic drugs in responders from six months after the initiation of the ketogenic diet.

\section{Usual care}

Patients randomized to usual care will continue to take their anti-epileptic drugs and no changes will be made to the anti-epileptic drugs treatment. Since a ketogenic diet is a treatment of last resort, the children in the usual care group will also receive a ketogenic diet after a delay of four months. The controls will be treated and monitored according to good clinical practice; however, this is not part of our proposed study.

\section{Timeline of the study}

The timeline of the study is shown in Figure 5.2. Patients in the intervention group will visit the multidisciplinary team (nurse practitioner, dietician, pediatrician and neurologist) at time points T0 (at baseline), T1 (during admission), T2 (6 weeks after admission), T3 (4 months after admission), FU1 (3 months follow-up), FU2 (6 months follow-up), FU3 (9 months follow-up), and FU4 (12 months follow-up). At T0, T3, and FU4 a neurocognitive assessment will take place. An overview of measurements per time point is shown in Table 5.1. Patients in the intervention group will be asked to follow the diet during the complete study period and during follow-up, 16 months in total. 
Patients in the usual care group will visit the multidisciplinary team (nurse practitioner, pediatrician and neurologist) at time points T0, T2, and T3 and will have a neurocognitive assessment at T0 and T3. In case of drop-out, a visit with the multidisciplinary team and a neurocognitive assessment will take place.

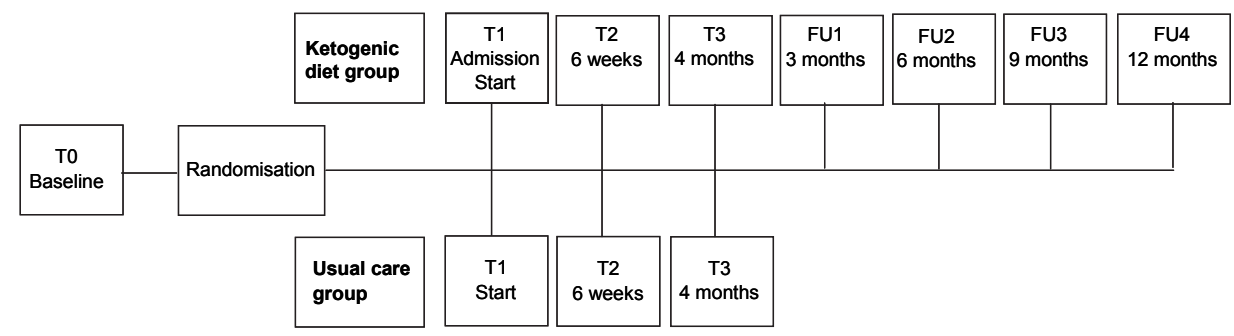

Figure 5.2 Timeline of the study.

T0: baseline; T1: admission; T2: 6 weeks after baseline period; T3: 4 months after baseline period; FU1: 3 months follow-up; FU2: 6 months follow-up; FU3: 9 months follow-up; FU12: 12 months follow-up.

\section{Effect evaluation}

Several instruments will be used to assess the effects of the ketogenic diet on the primary outcome (seizure frequency) and on secondary outcomes (seizure severity, side-effects, neurocognitive assessment and quality of life).

\section{Primary outcome}

Seizure frequency

A seizure calendar is used to record seizures. Types of seizures are described and labelled in accordance with ILAE classification. The number and type of seizures are recorded on the dates they occur.

\section{Secondary outcome}

Seizure severity

The National Hospital Seizure severity scale (NHS3) ${ }^{26}$ is a structured interview in which the clinician rater assigns a score to seizure severity based on interference with patient function. Information is gathered from the patient and witnesses, if available. Eight questions are asked about: tonic-clonic movements, falling, injury, incontinence, altered consciousness, postictal impairment, and disruptive automatisms.

Side-effects

Subjective complaints are assessed using the SIDAED (Side-effects of Anti-epileptic Drug), a list of 46 items with possible anti-epileptic drug-related complaints. The items included in the SIDAED form 10 categories: general Central Nervous System (CNS), 
behavior (increased irritability), depressive symptoms, changes in cognitive function, motor problems and coordination, visual complaints, headache, cosmetic and dermatological complaints, gastrointestinal complaints, and sexuality and menses. For each item the parents rate the severity of the complaint on a four-point Likert scale (no problem, mild, moderate, or serious problem). In addition, the duration of the complaints is scored (for a few weeks, for months or for a half year or longer).

Table 5.1 Overview of measurements per time point.

\begin{tabular}{|c|c|c|c|c|c|c|c|c|}
\hline Measurement & TO & T1* & T2 & T3 & FU1* & FU2* & FU3* & FU4* \\
\hline Demographic characteristics & $x$ & & & & & & & \\
\hline Clinical measurement & $x$ & $x$ & $x$ & $\mathrm{x}$ & $x$ & $x$ & $x$ & $x$ \\
\hline National Hospital Seizure severity scale & $x$ & $x$ & $x$ & $x$ & $x$ & $x$ & $x$ & $x$ \\
\hline Side-effects of Anti-epileptic Drug & $x$ & $x$ & $x$ & $x$ & $x$ & $x$ & $x$ & $x$ \\
\hline Credibility And Expectancy Questionnaire & $\mathrm{x}$ & & & & & & & \\
\hline Peabody Picture Vocabulary test & $x$ & & & $x$ & & & & $x$ \\
\hline Beery Developmental Visual-Motor Integration test & $x$ & & & $x$ & & & & $x$ \\
\hline FEPSY computerized test & $\mathrm{x}$ & & & $x$ & & & & $x$ \\
\hline Kaufman Assessment Battery & $x$ & & & $x$ & & & & $x$ \\
\hline Actigraphy & $x$ & & & $x$ & & & & $x$ \\
\hline SEV Questionnaire & $\mathrm{x}$ & & & $x$ & & & & $x$ \\
\hline SDQ Questionnaire & $x$ & & & $x$ & & & & $x$ \\
\hline POMS Questionnaire & $x$ & & & $x$ & & & & $x$ \\
\hline The Personal Adjustment and Role Skills Scale & $x$ & & & $x$ & & & & $x$ \\
\hline The Hague Restrictions in Childhood Epilepsy Scales & $\mathrm{x}$ & & & $x$ & & & & $x$ \\
\hline EuroQol & $x$ & & & $x$ & & & & $x$ \\
\hline TAPQOL or TACQOL & $x$ & & & $x$ & & & & $x$ \\
\hline Blood & $x$ & & $x$ & $x$ & $x$ & $x$ & $x$ & $x$ \\
\hline Urine & $x$ & & $x$ & $x$ & $x$ & $x$ & $x$ & $x$ \\
\hline Keton bodies measurement & $x$ & $x$ & $x$ & $x$ & $x$ & $x$ & $x$ & $x$ \\
\hline ECG & $x$ & & & $x$ & $x$ & $x$ & $x$ & $x$ \\
\hline
\end{tabular}

T0: baseline; T1: admission; T2: 6 weeks after baseline period; T3: 4 months after baseline period; FU1: 3 months follow-up; FU2: 6 months follow-up; FU3: 9 months follow-up; FU12: 12 months follow-up;

* intervention group only.

\section{Neurocognitive assessment}

\section{Peabody Picture Vocabulary test}

The Peabody Picture Vocabulary test (PPVT-III-NL: Dutch version) is an individually administered measure of receptive vocabulary. ${ }^{27}$ The test can be administered beginning at the age of $21 / 2$ years. The child listens to the examiner's stimulus word and must choose the picture that best describes the word from a 4-picture multiple choice array. The score on this test can be interpreted as an achievement test of the child's vocabulary that does not require a verbal response and as a screening measure of verbal ability. There is supportive data for the validity of the PPVT-III as a measure for global cognitive intelligence. 
Beery Developmental Visual-Motor Integration test

The Beery Developmental test of Visual-Motor Integration (Beery-VMI) is a widely used assessment of psychomotor development. It measures problems with visual perception, motor coordination, and visual motor integration. The 30-item test can be administered from the age of 2 years. The Beery VMI was standardized on a national US sample of 1,737 individuals age 2 to 18 years (2010) and 1,021 adults ages 19-100 (2006), and has proven reliability and validity. ${ }^{28}$

FEPSY computerized test

The FEPSY is a neurocognitive computerized test battery consisting of different subtests. For this study we use the simple auditory and visual reaction times, in which the stimulus exposure endures until a push-button (space-bar) response is given. ${ }^{29}$ Simple reaction tests give information on alertness functions and on the speed at which the information processing system is activated. We also use the binary choice reaction task in which a combination of accuracy and speed of responses are measured. This test measures the speed of central information processing. Finally, we use the finger tapping task in which motor activation and fluency is measured. In general, these tests can be administered from the age of 6 .

\section{Kaufman Assessment Battery}

The Kaufman Assessment Battery for Children II (K-ABC II) is used to investigate the level of information processing capacities of children beginning at the age of $21 / 2$ years. ${ }^{30}$ Two subtests will be administered: (1) Number recall, which is a measure of sequential processing and short term memory. The examiner says a series of numbers and the child repeats them. Digit span forward is considered a measure of efficiency of attention or "freedom from distractibility" and (2) conceptual thinking, which is a measure of nonverbal reasoning skills. The child looks at pictures and determines the one that does not belong.

\section{Actigraphy}

During the administration of the PPVT-III and the K-ABCII test, an actigraphic device (Actiwatch ${ }^{\circledR}$ model AW4: Cambridge Neurotechnology Ltd., United Kingdom) will be used to assess motor activity as a measure of alertness. As some of the children in the design are not testable with the described cognitive tests we decided to use an assessment of motor activity in a standardized situation. A wrist watch will be used that translates activity into movement of the watch hand. Registration takes place in a standardized way during the first 10 minutes of the Peabody Picture Vocabulary test and the first 5 minutes of the K-ABC-II test. When patients are not eligible to perform the neurocognitive assessment, the wrist watch will be used for 15 minutes during the visit at the nurse practitioner's office while watching a DVD with songs and pictures. 
SEV Questionnaire

The SEV questionnaire (Sociaal Emotionele Vragenlijst: Social Emotional Questionnaire) is a DSM-IV oriented questionnaire which is used to assess four domains of behavioral and social emotional dysfunction. These domains are: Attention Deficit Hyperactivity Disorders, Oppositional Defiant behavior and Conduct Disorders, Anxiety and Depression, and Autism spectrum disorders. The questionnaire consists of 72 items describing problem behaviour. ${ }^{31}$

SDQ Questionnaire

The Strengths and Difficulties Questionnaire (SDQ) is a brief behavioral screening questionnaire that provides balanced coverage of children and young people's behaviors, emotions, and relationships. ${ }^{32}$ The SDQ is applicable to children beginning at the age of 4 and asks about 25 attributes that are divided into five relevant dimensions: namely conduct problems, emotional symptoms, hyperactivity, peer relationships, and pro-social behavior.

\section{POMS Questionnaire}

The Profile of Mood States (POMS) has been developed to identify and assess seven transient, fluctuating affective mood states: Tension-Anxiety, Depression-Dejection, Anger-Hostility, Vigor-Activity, Fatigue-Inertia, Friendliness, and ConfusionBewilderment. ${ }^{33}$ The questionnaire contains 65 items. All items describe an emotional state which can be rated on a 5-point scale ranging from "not at all" to "extremely".

The Personal Adjustment and Role Skills Scale (PARS)

The Personal Adjustment and Role Skills Scale, 3rd edition (PARS-III) was specifically developed to measure psychosocial adjustment in children with chronic physical illness. ${ }^{34}$ This instrument is a brief parent-completed index of youth psychosocial adjustment. The instrument yields six factor-derived psychosocial subscales: namely peer relations, dependency, hostility, productivity, anxiety/depression, and withdrawal. The PARS-III is a reliable and valid index of youth psychosocial adjustment and can be used for both clinical screening and research purposes. ${ }^{35}$

The Hague Restrictions in Childhood Epilepsy Scales (HARCES)

The HARCES is a questionnaire consisting of 11 items used to assess impairments in daily functioning that are related to epilepsy, e.g. how much influence does the epilepsy have on your child's freedom of functioning in the house? Items are answered on a 4-point scale ranging from "no impairment" to "very much impairment". ${ }^{36}$

Credibility And Expectancy questionnaire (CEQ)

The Credibility and Expectancy Questionnaire (CEQ) ${ }^{37}$ is a multidimensional selfreported instrument of 6 items, rated on a 9- or 10-point Likert-scale, meant to 
measure the expectancy and credibility a person has about the therapy he or she will receive. Its sound psychometric properties have been proven. We will measure the credibility and expectancy for the treatment of both the children and their parents.

\section{Generic quality of life}

\section{EuroQol}

Living with uncontrolled seizures has a negative impact on the quality of life of the child and his/her parents. Therefore, we will measure generic quality of life of both the children and their parents. For this purpose we will use the EuroQol instrument for children of 12 years and older developed by the EuroQol group. ${ }^{38}$ For younger children, we will use a version of the EuroQol developed for children (EuroQol-Youth) or proxies. In accordance with the regular EuroQol, the EuroQol-Youth is comprised of the following 5 dimensions: mobility, self-care, usual activities, pain/discomfort and anxiety/depression. Each dimension has 3 levels: no problems, some problems and severe problems, thus defining $243\left(3^{5}\right)$ possible health states. 'Unconscious' and 'dead' have been added to these states, resulting in a total of 245 states. Furthermore, the EQ-5D consists of a visual analogue scale (VAS) ranging from zero (worst imaginable health state) to 100 (best imaginable health state).

\section{TAPQOL or TACQOL}

QOL will also be assessed using the TAPQOL (TNO-AZL Preschool Children's Quality of Life) for children aged between 1-5 years ${ }^{39}$ and the TACQOL (TNO-AZL Children's Quality of life) for children aged between 6-16 years. ${ }^{40}$ There are 12 dimensions in the TAPQOL: lungs, stomach, skin, sleep, appetite, aggressive behavior, positive emotions, fear, vitality, social behavior, motor functioning and communication. All dimensions are scored on a scale from $0-100$. The TACQOL is comprised of 7 dimensions: bodily complaints, independence, motor functioning, cognition, social functioning, positive and negative emotions. The scores of the first 5 dimensions vary from 0-32 and the scores of the last two dimensions from 0-16.

\section{Economic evaluation}

The economic evaluation will be performed from the societal perspective, which implies that all relevant costs and effects will be taken into account. The economic evaluation compares costs and effects of the ketogenic diet in comparison with usual care. A Cost-Effectiveness Analysis (CEA) and a Cost-Utility Analysis (CUA) will be performed. Outcomes of interest for the CEA and the CUA will be the reduction of seizures and improvement in the Quality Adjusted Life Years (QALYs) respectively. The QALY is a measure of disease burden, including both the quality (utilities) and the quantity of life lived. Total societal costs will be calculated based on the Dutch guidelines for cost calculations in health care. We distinguish four cost categories: intervention costs, health care sector costs, costs for the patient and family, and 
productivity costs. For this study, three instruments are used to measure the costs and utilities.

\section{Prospective cost diary}

The diary is used to identify all relevant cost aspects with respect to health care sector costs and patient and family costs. ${ }^{41}$ Each diary covers a period of four weeks and will be filled out during the 4 week baseline period and during the 4 month study period. The intervention group will also fill out the diary during the 12 months of follow-up. Total costs will be estimated using a bottom-up approach, where information on each element of service used will be multiplied by an appropriate standardized unit cost and be summed to provide an overall total cost. For the cost valuation, standardized cost prices from the Dutch manual for cost analysis in health care research will be used. $^{42}$

\section{Productivity losses}

A retrospective questionnaire and the patient modules of the PROductivity and DISease Questionnaire (PRODISQ) ${ }^{43}$ will be used to measure production losses of the patient's parents. Productivity costs will be calculated by means of the friction cost method, based on a mean added value of the Dutch working population. This method takes into account production losses confined to the period needed to replace a sick employee.

\section{Utilities (EQ-5D)}

Both generic quality of life, as well as utilities, will be derived by means of the EuroQol, as mentioned before. Utility values will be calculated for all health states of the EuroQol, using preferences from the UK general population value set which provides an algorithm as a series of decrements from 1 , the value of full health. Values of the UK population for the scoring function of the health states described by the EQ5D were measured with the Time Trade Off (TTO) technique (see Torrance, 1976) on a random sample of approximately 3000 members of the adult population of the UK. ${ }^{44}$ The utility values derived from the Dolan algorithm will be used to compute Quality Adjusted Life Years (QALYS). However, to overcome the tariff's differences between countries, the Dutch tariff is also used to calculate utilities in the sensitivity analysis. Lamers, ${ }^{45}$ calculated by means of the TTO method a Dutch scoring function for the health states described by the EQ-5D, based on a Dutch population sample of 300 people. 


\section{Analyses}

\section{I) Effect evaluation}

Our primary (base-case) analyses will be performed according to the intention-totreat principle, including data from all participants regardless of whether they received the intervention or not. For the analyses we will use SPSS statistical software. Respondents for whom at least $75 \%$ of the data per measurement instrument are available will be included in the analysis. Missing data on an item level will be handled using SPSS missing value regression analysis. Completely missing measurements will be handled using multiple imputation. Between-group differences in proportions (dichotomous variables) will be tested using the Chi-square test and between-group differences in means (continuous variables) will be tested using Student's t-test for independent samples. In addition, a multivariate regression analysis will be performed with the covariates of sex, age, severity of disease, duration of disease etc. The accuracy of the findings will be expressed in terms of $95 \%$ confidence intervals.

\section{Ila) Trial-based economic evaluation}

A baseline analysis will be performed to examine the comparability of groups at baseline for both costs and outcomes. If necessary, methods will be applied to control for differences in baseline. ${ }^{46}$ A Kolmogorov-Smirnov test will be performed to investigate whether data are distributed normally. Despite the usual skewness in the distribution of costs, the arithmetic means will generally be considered the most appropriate measures for describing cost data. ${ }^{47,48}$ Therefore, arithmetic means (and standard deviations) will be presented. In case the cost data are skewed, nonparametric bootstrapping will be used to test for statistical differences in costs between the intervention and usual care. The bootstrap replications will be used to calculate $95 \%$ confidence intervals around the costs $(95 \% \mathrm{Cl})$, based on the 2.5 th and 97.5th percentiles. If cost data are distributed normally, t-tests will be used. The incremental cost effectiveness ratio (ICER) will be determined on the basis of the incremental costs and effects of the ketogenic diet in comparison with a waiting-list group. The cost-effectiveness ratio will be stated in terms of costs per outcome rate (decrease in seizure frequency and severity), and the cost-utility ratio will focus on the net cost per utility gained.

The robustness of the ICER will be checked by non-parametric bootstrapping. Bootstrap simulations will also be conducted in order to quantify the uncertainty around the ICER, yielding information about the joint distribution of cost and effect differences. The bootstrapped cost-effectiveness ratios will be plotted subsequently in a cost effectiveness plane. The choice of treatment depends on the maximum amount of money that society is prepared to pay for a gain in effectiveness, which is called the ceiling ratio. Therefore, the bootstrapped ICERs will also be depicted in a costeffectiveness acceptability curve showing the probability that the ketogenic diet is 
cost-effective using a range of ceiling ratios. In addition, to demonstrate the robustness of our base-case findings, multi-way sensitivity analyses will be performed. $^{49}$

\section{IIb) Model-based economic evaluation}

The time horizon of the Cost Utility Analysis (CUA) will be extrapolated towards the remaining life expectancy of the study population. The CUA is of major importance since the impact of a ketogenic diet on seizure reduction, costs, and QOL reaches beyond the 4-month study period of the clinical study. A Markov Monte Carlo decision analytic model will be developed to calculate lifetime incremental costs and incremental QALYs of treatment with a ketogenic diet in comparison with treatment with antie-pileptic drugs only. Markov models assume that a patient is always in one of a finite number of health states. All events are represented as transitions from one state to another. A first-order Monte Carlo evaluation of a Markov model determines the prognoses of a large number of individual patients. The time horizon of the analysis is divided into cycles. Each patient begins in an initial state. During each cycle, the patient may make a transition from one state to another according to the laws of chance, as dictated by the transition probabilities. After the first patient has completed the simulation, another patient begins in the initial state and a new simulation is performed. This process is repeated a very large number of times, and each simulation generates a quality adjusted survival time and costs. Monte Carlo analysis, as opposed to a Markov cohort model without memory, offers the possibility to flag subjects in order to track their characteristics and disease histories; this is a very flexible approach to modelling variability within a population. The model will combine the results of the clinical study and data from the medical literature. In the modelling study we will also perform probabilistic sensitivity analysis to test parameter uncertainty and to construct cost-effectiveness acceptability curves. Future costs and effects will be discounted according to the Dutch guidelines for cost calculations in health care. $^{42}$

\section{Discussion}

Our design is aimed at assessing the effectiveness and cost-effectiveness of a ketogenic diet among children and adolescents with intractable epilepsy. It is a prospective RCT comparing the ketogenic diet with usual care.

The ketogenic diet can be a treatment of last resort for patients with intractable epilepsy. This alternative therapy is used only in the minority of children who could potentially benefit from it. In order to optimize therapy for children with uncontrolled seizures, the ketogenic diet should be prescribed for more children with intractable epilepsy. 


\section{Limitations and complications}

Compliance with a ketogenic diet is difficult due to its restrictive nature. Unfortunately, non-compliance limits the intended effect and increases the costs to society, resulting in a less favorable cost-effectiveness ratio. In order to overcome this problem, the children and adolescents with uncontrolled epilepsy who are on the ketogenic diet will be monitored according to a strict standardized protocol. Therefore it is likely that the efficiency of patient care will improve. However, a formal assessment of the diet's cost-effectiveness has not yet been performed and is the focus of the present proposal.

\section{Conclusion}

Growing up with seizures affects the child's personality and cognitive development and interferes with many aspects of everyday life, including learning at school, leisure and occupational activities. Epilepsy has been shown to have a high impact on quality of life when children have intractable seizures and additional disabilities.

This study will provide information about the cost-effectiveness of the ketogenic diet, its effects on clinical outcomes, on neurocognitive functioning and on quality of life. Our study is the first study assessing the cost-effectiveness of a ketogenic diet. 


\section{References}

1. Fisher RS, van Emde Boas W, Blume W, Elger C, Genton P, Lee P, Engel J Jr. Epileptic seizures and epilepsy: definitions proposed by the International League Against Epilepsy (ILAE) and the International Bureau for Epilepsy (IBE). Epilepsia. 2005;46:470-2.

2. Strzelczyk A1, Reese JP, Dodel R, Hamer HM. Cost of epilepsy: a systematic review. Pharmacoeconomics. 2008;26:463-76.

3. Leonardi M, Ustun TB. The global burden of epilepsy. Epilepsia. 2002;43(Suppl 6):21-5.

4. Forsgren L, Beghi E, Oun A, Sillanpää M. The epidemiology of epilepsy in Europe - a systematic review. Eur J Neurol. 2005;12:245-53.

5. Pugliatti M, Beghi E, Forsgren L, Ekman M, Sobocki P. Estimating the cost of epilepsy in Europe: a review with economic modeling. Epilepsia. 2007;48:2224-33.

6. Poos MJJC, Smit JM, Groen J, Kommer GJ, SLobbe LCJ: Kosten van ziekten in Nederland 2005. RIVM 2008.

7. Hoeymans N, Melse JM, Schoenmaker CG: Gezondheid en determinanten. Deelrapport van de Volksgezondheid Toekomst Verkenning 2010 Van gezond naar beter. RIVM-rapport nr. 270061006 2010.

8. Kossoff EH, Zupec-Kania BA, Amark PE, Ballaban-Gil KR, Christina Bergqvist AG, Blackford R, Buchhalter JR, Caraballo RH, Helen Cross J, Dahlin MG, Donner EJ, Klepper J, Jehle RS, Kim HD, Christiana Liu YM, Nation J, Nordli DR Jr, Pfeifer HH, Rho JM, Stafstrom CE, Thiele EA, Turner Z, Wirrell EC, Wheless JW, Veggiotti P, Vining EP; Charlie Foundation, Practice Committee of the Child Neurology Society; Practice Committee of the Child Neurology Society; International Ketogenic Diet Study Group. Optimal clinical management of children receiving the ketogenic diet: recommendations of the International Ketogenic Diet Study Group. Epilepsia. 2009;50:304-17.

9. Freeman J, Veggiotti P, Lanzi G, Tagliabue A, Perucca E; Institute of Neurology IRCCS C. Mondino Foundation. The ketogenic diet: from molecular mechanisms to clinical effects. Epilepsy Res. 2006;68:145-80.

10. Kossoff EH, McGrogan JR, Bluml RM, Pillas DJ, Rubenstein JE, Vining EP. A modified Atkins diet is effective for the treatment of intractable pediatric epilepsy. Epilepsia. 2006;47:421-4.

11. Zupec-Kania BA, Spellman E. An overview of the ketogenic diet for pediatric epilepsy. Nutr Clin Pract. 2008;23:589-96.

12. Caraballo RH, Cersósimo RO, Sakr D, Cresta A, Escobal N, Fejerman N. Ketogenic diet in patients with Dravet syndrome. Epilepsia. 2005;46:1539-44.

13. Coppola G, Veggiotti P, Cusmai R, Bertoli S, Cardinali S, Dionisi-Vici C, Elia M, Lispi ML, Sarnelli C, Tagliabue A, Toraldo C, Pascotto A. The ketogenic diet in children, adolescents and young adults with refractory epilepsy: an Italian multicentric experience. Epilepsy Res. 2002;48:221-7.

14. Freeman JM, Vining EP, Pillas DJ, Pyzik PL, Casey JC, Kelly LM. The efficacy of the ketogenic diet-1998: a prospective evaluation of intervention in 150 children. Pediatrics. 1998;102:1358-63.

15. Kang HC, Kim YJ, Kim DW, Kim HD. Efficacy and safety of the ketogenic diet for intractable childhood epilepsy: Korean multicentric experience. Epilepsia. 2005;46:272-9.

16. Kankirawatana $\mathrm{P}$, Jirapinyo $\mathrm{P}$, Kankirawatana $\mathrm{S}$, Wongarn $\mathrm{R}$, Thamanasiri N. Ketogenic diet: an alternative treatment for refractory epilepsy in children. J Med Assoc Thai. 2001;84:1027-32.

17. Kossoff EH, Pyzik PL, McGrogan JR, Vining EP, Freeman JM. Efficacy of the ketogenic diet for infantile spasms. Pediatrics. 2002;109:780-3.

18. Kossoff EH, Thiele EA, Pfeifer HH, McGrogan JR, Freeman JM. Tuberous sclerosis complex and the ketogenic diet. Epilepsia. 2005;46:1684-6.

19. Maydell BV, Wyllie E, Akhtar N, Kotagal P, Powaski K, Cook K, Weinstock A, Rothner AD. Efficacy of the ketogenic diet in focal versus generalized seizures. Pediatr Neurol. 2001;25:208-12.

20. Vining EP, Freeman JM, Ballaban-Gil K, Camfield CS, Camfield PR, Holmes GL, Shinnar S, Shuman R, Trevathan E, Wheless JW. A multicenter study of the efficacy of the ketogenic diet. Arch Neurol. 1998;55:1433-7.

21. Keene DL. A systematic review of the use of the ketogenic diet in childhood epilepsy. Pediatr Neurol. 2006;35:1-5. 
22. Lefevre F, Aronson N. Ketogenic diet for the treatment of refractory epilepsy in children: A systematic review of efficacy. Pediatrics. 2000;105:E46.

23. Levy R, Cooper P. Ketogenic diet for epilepsy. Cochrane Database Syst Rev 2003;3: CD001903.

24. Sinha SR, Kossoff EH. The ketogenic diet. Neurologist. 2005;11:161-70.

25. Neal EG, Chaffe H, Schwartz RH, Lawson MS, Edwards N, Fitzsimmons G, Whitney A, Cross JH. The ketogenic diet for the treatment of childhood epilepsy: a randomised controlled trial. Lancet Neurol. 2008;7:500-6.

26. O'Donoghue MF, Duncan JS, Sander JW. The National Hospital Seizure Severity Scale: a further development of the Chalfont Seizure Severity Scale. Epilepsia. 1996;37:563-71.

27. Dunn LM, Dunn LM. Peabody Picture Vocabulary Test-III-NL, Nederlandse versie door Liesbeth Schlichting. Harcourt Assessment B.V., Amsterdam; 2005.

28. Beery K. The Beery-Buktenica Developmental Test of Visual-Motor Integration: VMI with Supplemental Developmental Test of Visual Perception and Motor Coordination: Administration, Scoring and Teaching Manual. Parsippany, NJ: Modern Curriculum Press; 1997.

29. Alpherts WC, Aldenkamp AP. Computerized neuropsychological assessment of cognitive functioning in children with epilepsy. Epilepsia. 1990;31(Suppl 4):S35-40.

30. Kaufman AS, Kaufman NL. Kaufman Assessment Battery for Children (K-ABC). Publishing, Circle Pines; 1983.

31. Scholte EM, van der Ploeg JD. Handleiding Sociaal-Emotionele Vragenlijst (SEV). Houten: Bohn Stafleu van Loghum; 2007.

32. Berkel A, et al. Handleiding voor het gebruik van de SDQ binnen de jeugdgezondheidszorg. Vragenlijst voor het signaleren van psychosociale problemen bij kinderen van 7-12 jaar. Zutphen: Markant congressen 2006.

33. MC Nair DM, Lorr M, Droppleman Lf. Profile of Mood States: Manual San Diego, CA: Educational and Industrial Testing Service; 1971.

34. Walker DK, Stein RE, Perrin EC, Jessop DJ. Assessing psychosocial adjustment of children with chronic illnesses: a review of the technical properties of PARS III. J Dev Behav Pediatr. 1990;11:116-21.

35. Hendriksen JG, Poysky JT, Schrans DG, Schouten EG, Aldenkamp AP, Vles JS. Psychosocial adjustment in males with Duchenne muscular dystrophy: psychometric properties and clinical utility of a parentreport questionnaire. J Pediatr Psychol. 2009;34:69-78.

36. Carpay HA, Vermeulen J, Stroink H, Brouwer OF, Peters AC, van Donselaar CA, Aldenkamp AP, Arts WF. Disability due to restrictions in childhood epilepsy. Dev Med Child Neurol. 1997;39:521-6.

37. Devilly GJ, Borkovec TD. Psychometric properties of the credibility/ expectancy questionnaire. J Behav Ther Exp Psychiatry. 2000;31:73-86.

38. EuroQol-a new facility for the measurement of health-related quality of life. The EuroQol Group. Health Policy. 1990;16:199-208.

39. Fekkes M, Theunissen NC, Brugman E, Veen S, Verrips EG, Koopman HM, Vogels T, Wit JM, VerlooveVanhorick SP. Development and psychometric evaluation of the TAPQOL: a health-related quality of life instrument for 1-5-year-old children. Qual Life Res. 2000;9:961-72.

40. Vogels T, Verrips GH, Koopman HM. TACQOL manual. Parent Form and Child Form Leiden: Leiden Center for Child Health and Pediatrics LUMC-TNO; 2000.

41. Goossens ME, Rutten-van Mölken MP, Vlaeyen JW, van der Linden SM. The cost diary: a method to measure direct and indirect costs in cost-effectiveness research. J Clin Epidemiol. 2000;53:688-95.

42. Oostenbrink JB, et al. Handleiding voor kostenonderzoek, methoden en standaard kostprijzen voor economische evaluaties in de gezondheidszorg. College voor zorgverzekeringen 2004.

43. Koopmanschap MA. PRODISQ: a modular questionnaire on productivity and disease for economic evaluation studies. Expert Rev Pharmacoecon Outcomes Res 2005, 5(1):23-8.

44. Dolan P, Gudex C, Kind P, Williams A. The time trade-off method: results from a general population study. Health Econ. 1996;5:141-54.

45. Lamers LM, Stalmeier PF, McDonnell J, Krabbe PF, van Busschbach JJ. Measuring the quality of life in economic evaluations: the Dutch EQ-5D tariff. Ned Tijdschr Geneeskd. 2005;149:1574-8.

46. Manca A, Hawkins N, Sculpher MJ. Estimating mean QALYs in trial-based cost-effectiveness analysis: the importance of controlling for baseline utility. Health Econ. 2005;14:487-96.

47. Barber JA, Thompson SG. Analysis of cost data in randomized trials: an application of the nonparametric bootstrap. Stat Med. 2000;19:3219-36. 
48. Ramsey S, Willke R, Briggs A, Brown R, Buxton M, Chawla A, Cook J, Glick H, Liljas B, Petitti D, Reed S. Good research practices for cost-effectiveness analysis alongside clinical trials: the ISPOR RCT-CEA Task Force report. Value Health. 2005;8:521-33.

49. Briggs $A H$, Wonderling DE, Mooney CZ. Pulling cost-effectiveness analysis up by its bootstraps: a nonparametric approach to confidence interval estimation. Health Econ. 1997;6:327-40. 


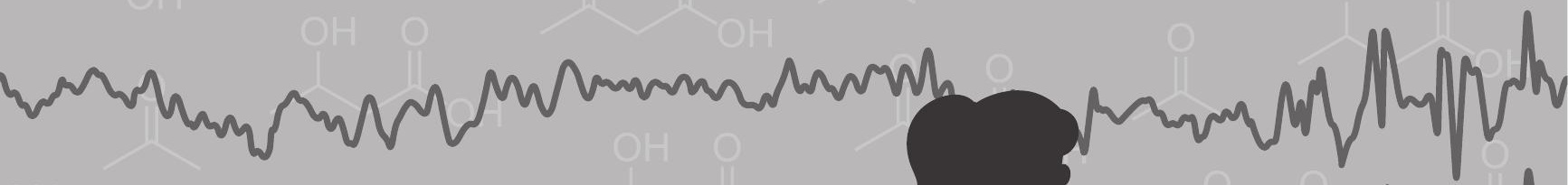

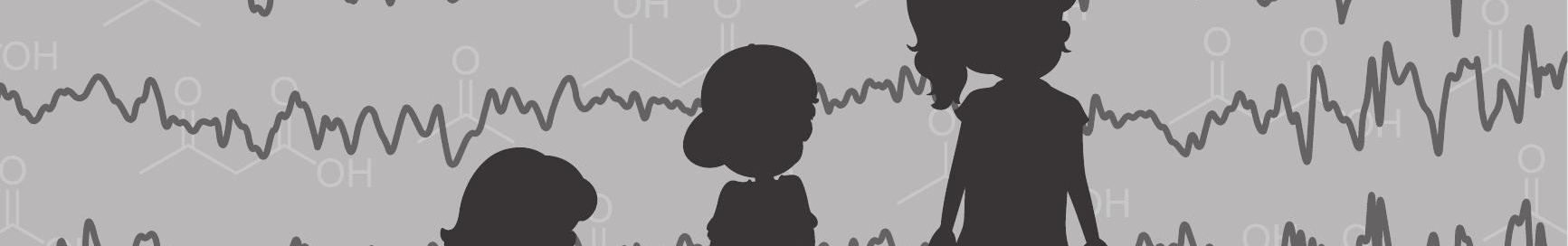




\section{Chapter 6}

\section{A randomized controlled trial of the ketogenic diet in refractory childhood epilepsy}




\section{Abstract}

\section{Objective}

To evaluate the efficacy and tolerability of the ketogenic diet (KD) during the first 4 months of a Randomized Controlled Trial (RCT) in refractory epilepsy patients aged 1-18 years.

\section{Methods}

Children and adolescents with refractory epilepsy, not eligible for epilepsy surgery, were included. Following one month at baseline, patients were randomized to either the KD or to care as usual (CAU).

Primary outcome is the proportion of patients with at least $50 \%$ reduction in seizure frequency at four months. Secondary outcomes are mean percentage of baseline seizures, seizure severity and side-effects.

\section{Results}

57 Patients were randomized; nine dropped out, leaving 48 for analysis (i.e. 26 KD, 22 CAU). In an intention-to-treat (ITT) analysis, 13 patients (50\%) treated with the KD and four patients (18.2\%) of the CAU group were responders.

Mean seizure frequency at 4 months compared to baseline, after removal of 2 outliers in the KD group, was significantly lower $(p=0.024)$ in the KD group $56 \%(95 \% \mathrm{Cl}: 36-76)$ than in the CAU group 99\% (95\% Cl: 65-133\%).

Twice as many patients in the KD group had a relevant decrease in seizure severity score $(p=0.070)$.

Patients treated with the KD had a significantly higher score for gastrointestinal symptoms $(p=0.021)$ without an increase in the total score of side-effects.

\section{Conclusions}

This trial provides class I evidence that the KD is an effective therapy in children and adolescents with refractory epilepsy compared with CAU. Most often reported side-effects are gastro-intestinal symptoms. 


\section{Introduction}

In refractory epilepsy patients in whom resective epilepsy surgery is not feasible, nonpharmacological treatment options, including the ketogenic diet (KD), can be considered. ${ }^{1}$

The KD is a high-fat, low-carbohydrate diet of which there are various forms.

The classical KD consists of long-chain triglycerides (LCTs), usually applied in a KD-ratio of $4: 1$ or 3:1 for [fat]:[proteins and carbohydrates]. Another well-known form of the KD is the MCT-diet, consisting mainly of medium-chain triglycerides (MCTs). ${ }^{2}$

In a recent Cochrane review, authors concluded that, despite heterogeneity, all trials showed that at least $38 \%$ of the patients on the KD had a $50 \%$ reduction in seizures compared to controls at 3 months, and that this response was maintained for up to a year. The main reasons for drop-outs were gastrointestinal side-effects (30\%) and dislike of the diet. ${ }^{3}$ Two RCTs on the efficacy of the KD have been published: Neal et al. on the classical and MCT KD, and Sharma et al. on the modified Atkins diet (MAD). ${ }^{2,4,5}$ The RCT by Neal et al. showed that the classical diet did not have any advantage over MCT-diet in terms of efficacy and tolerability. ${ }^{2}$ Seizure severity was not measured in any of the RCTs.

To gain more insight into the effectiveness of the KD and evaluate its influence on seizure severity and side-effects, the current RCT was performed in children and adolescents with refractory epilepsy. This article describes the results of a 4-month study period.

\section{Methods}

This study was carried out within a tertiary referral center for epilepsy (Kempenhaeghe, Heeze, The Netherlands). Patients were included during the period, July 2010 until August 2014. The study was approved by the Medical Ethics Committee according to Dutch Governmental Guidelines. Parents and, if appropriate, children also gave written informed consent.

\section{Study design}

The timeline of the study is presented graphically in Figure 6.1. A comprehensive overview of the trial design can be found elsewhere. ${ }^{6}$ Briefly, patients were randomized to either the KD or to CAU after a one-month baseline period. A software package (ALEA) was used to support the randomization which was based on the minimization method.

CAU is defined as the child continuing to take his or her anti-epileptic drugs (AEDs). Patients randomized into the KD group were studied during a 4-month period and followed-up for a further 12 months. Patients randomized into the CAU group were 
treated with the KD according to good clinical practice after a delay of 4 months. Primary outcome is the proportion of patients with a seizure frequency reduction of at least $50 \%$. Secondary outcome measures are mean number of seizures as a percentage of the number of seizures during the baseline period, seizure severity and side-effects.

Based on a minimum detectable difference in success rate of $35 \%$ between the KD group and $\mathrm{CAU}$, and assuming that alpha $=5 \%$ and power $=80 \%$, we needed 22 children for each group. ${ }^{6}$ A drop-out was defined as a child who drops out of the study before the first consultation with the neurologist which was scheduled six weeks after either initiating the KD or after randomization to CAU. Drop-outs identified by this definition were replaced by other eligible participants.

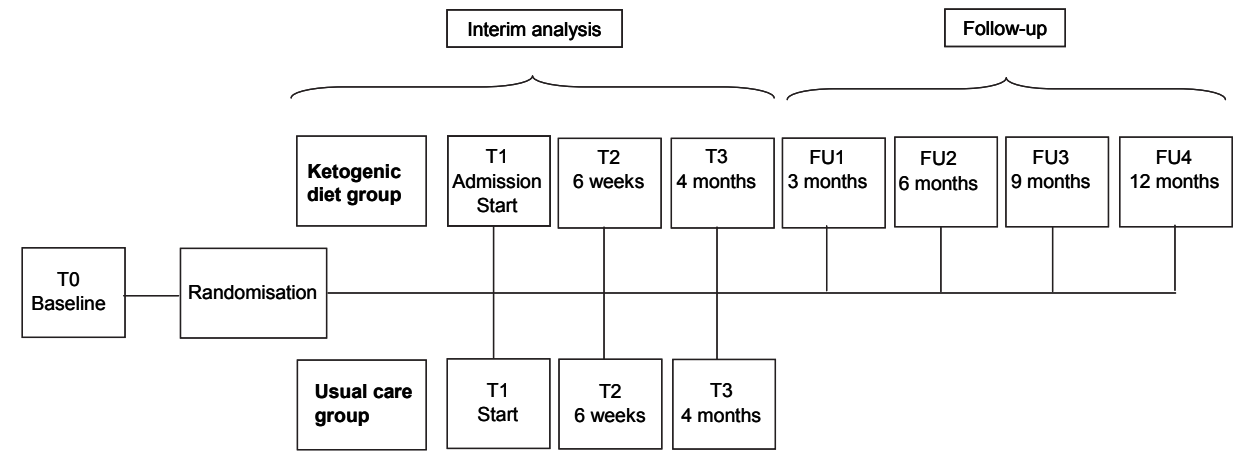

Figure 6.1 Flowchart study design.

\section{Patients}

Children and adolescents, aged between 1 and 18 years, with refractory epilepsy not eligible for epilepsy surgery, were included. Refractory epilepsy was defined as seizures not adequately controlled by optimal treatment with $\geq 2$ AEDs. ${ }^{7}$ Patients were excluded if there were medical contra-indications or the expectation that compliance with the diet was not possible because of severe behavioral or motivational problems. ${ }^{6}$

For patients of both groups, changes in AED regime were only allowed if medically necessary.

\section{Ketogenic diet}

The KD was introduced according to the Dutch guideline ${ }^{8}$ during a 5-day hospitalization at the epilepsy center. The start of the diet was defined as the first change made to the patient's daily nutrition and the end of the diet as the first step in down-titration to a regular diet. 
The form applied most frequently was the MCT-diet. When only tube feeding was given, a liquid form of the classical KD was used.

\section{Outcome measures}

Out-patient assessments of seizure frequency, seizure severity, side-effects, blood and urine samples were carried out at baseline (T0), 6 weeks (T2) and 4 months (T3). An ECG was performed at TO and T3. Reasons for discontinuing the KD or trial were recorded.

\section{Seizure frequency}

Patients and/or caregivers were asked to record seizures on a seizure calendar during a 1-month baseline and the 4-month study period. A seizure cluster ( $\geq 5$ seizures in 15 minutes) was calculated as 1 seizure. Seizure frequency is expressed as a percentage change in the seizure frequency compared to 30-day baseline value. Mean seizure frequency at 6 weeks and for each of the 4 study months $(1,2,3$ and 4$)$ is expressed as a 30-day mean seizure frequency to be comparable with the baseline frequency. The patient was defined as a responder when the seizure frequency was reduced by $\geq 50 \%$ compared with the seizure frequency at baseline.

An intention-to-treat (ITT) analysis was performed. Missing data was handled by the last value carried forward principle.

\section{Seizure severity}

Seizure severity was assessed with the National Hospital Seizure Severity Scale (NHS3), ${ }^{9}$ a structured interview in which the clinician rater assigns a score to seizure severity based on interference with patient function. After defining most severe seizure type for each patient, scores were calculated for this and for all seizure types together. Values at T2 and T3 were compared with those at baseline. A difference of 2 points was estimated to be a clinically relevant change.

\section{Side-effects}

\section{SIDAED}

Side-effects were assessed using the Side-Effects of Anti-Epileptic Drugs (SIDAED) questionnaire, ${ }^{10}$ originally designed as a self-reporting questionnaire for adults with epilepsy. After obtaining permission from the authors, the questionnaire was adapted to a parent-reported child version; items on sexuality were excluded. Nine domains of side-effects are evaluated: general central nervous system, behavior/irritability, depressive symptoms, cognitive functions, motor problems/coordination, vision, headache, cosmetic and dermatological problems, and gastrointestinal function. The 
latter includes questions on weight changes, appetite, nausea and stomach trouble, diarrhea and obstipation. For each of the 43 items, the parents rate the severity of the complaint on a four-point Likert scale (no problem, mild, moderate, or serious problem).

\section{Anthropometry}

At each outpatient visit, growth and height were measured and processed using the growth analyser, version 3.5.197. Height-for-age and body mass index (BMI) were calculated and interpreted by the pediatrician of the KD team. A difference in standard deviation of $\geq 0.5$ for height-for-age and of $\geq 0.75$ for BMI were assumed clinically relevant.

\section{Lipid profile}

Fasting levels of total cholesterol, LDL cholesterol and triglycerides were measured in blood.

\section{Ketosis}

At time points T0, T2 and T3, beta-hydroxybutyrate (BHB) was measured in blood obtained by finger-prick. Urine samples were checked for ketone bodies.

At home, urine ketone levels were checked daily at the same time using a dipstick. If it proved impossible to obtain urine samples, BHB was measured three times a week. Levels of ketosis were recorded on the seizure calendar.

\section{Statistics}

Statistical analysis was performed using SPSS 21.0 for Windows.

Seizure reduction data were analyzed using Wilcoxon's signed-rank test for nonparametric data (two-tailed). Correlations between seizure reduction and blood $\mathrm{BHB}$ and urinary ketones were investigated using Spearman's rank correlation test. Between-group differences in proportions (i.e. responders vs. non-responders) were tested using the Chi-square test and between-group differences in means (i.e. percentage change in seizure frequency) were tested using Student's t-test for independent samples. Significance level was set at $p<0.05$. 


\section{Results}

A total of 58 patients were included in the study. Figure 6.2 graphically presents the flow of the patients. Table 6.1 summarizes the main demographic and clinical characteristics. Five of the seven patients treated with the classical KD, received this in fluid form via a percutaneous gastrostomy tube.

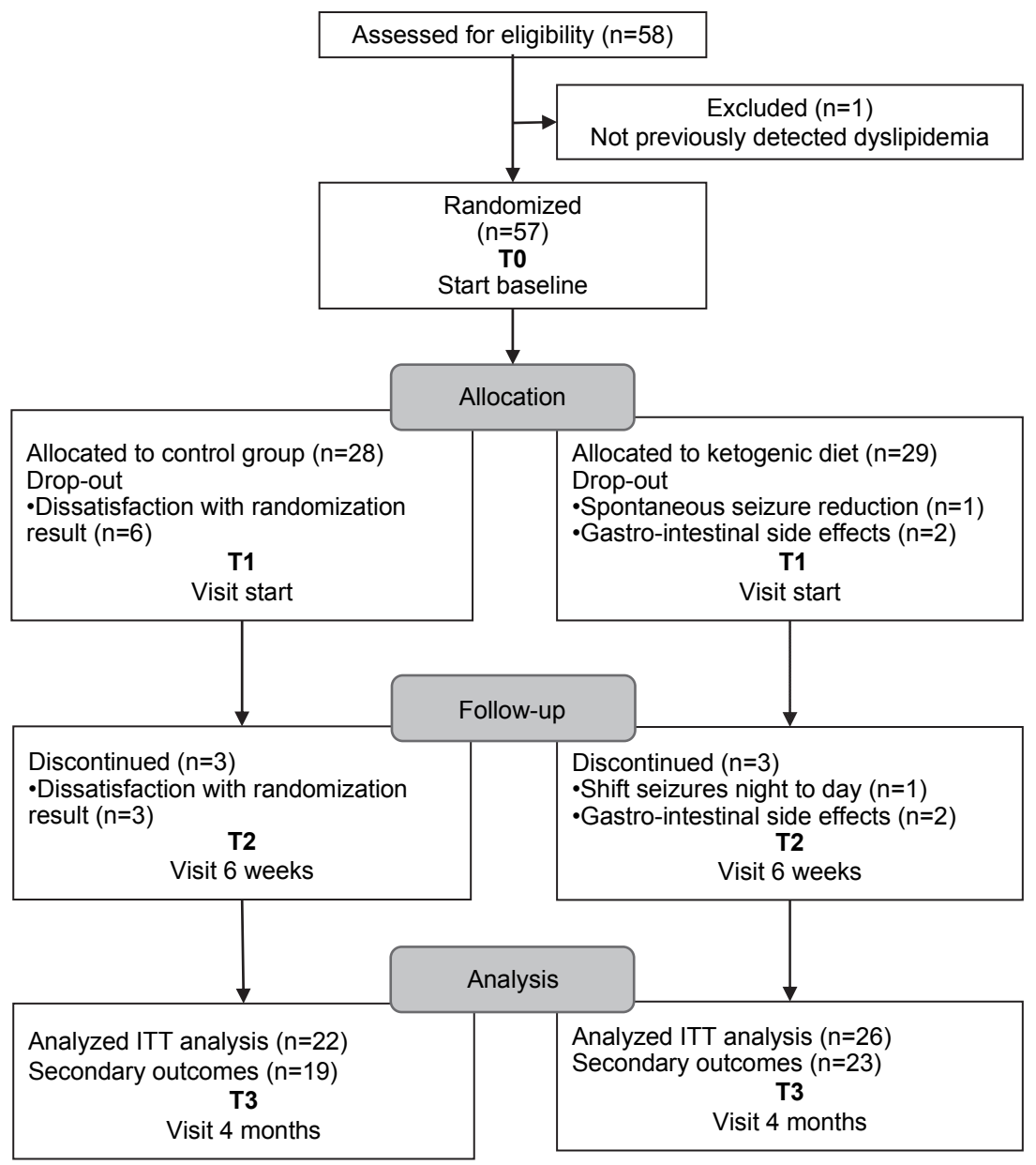

Figure 6.2 Flow of the patients. 
Table 6.1 Demography and clinical characteristics.

\begin{tabular}{|c|c|c|c|c|c|c|}
\hline & KD N=26 & \multicolumn{5}{|c|}{ CAU N=22 } \\
\hline & $\mathrm{N} \quad(\%)$ & Mean & (Min-max) & $\mathbf{N}(\%)$ & Mean & (Min-max) \\
\hline \multicolumn{7}{|l|}{ Gender } \\
\hline Male & $18(69.2)$ & & & 9 (40.9) & & \\
\hline Female & $8(30.8)$ & & & $13(59.1)$ & & \\
\hline \multicolumn{7}{|l|}{ Age (years;months) } \\
\hline at seizure onset & & $2 ; 4$ & $(0-8)$ & & $1 ; 9$ & $(0-10)$ \\
\hline at KD initiation or initiation trial & & $7 ; 8$ & $(2 ; 1-16 ; 5)$ & & $8 ; 1$ & $(1 ; 1-15 ; 7)$ \\
\hline$\leq 5$ years & $7(26.9)$ & & & $5(22.7)$ & & \\
\hline$>5-\leq 10$ years & $13(50.0)$ & & & $10(45.5)$ & & \\
\hline$>10-\leq 15$ years & $4(15.4)$ & & & $5(22.7)$ & & \\
\hline$>15-\leq 18$ years & $2(7.7)$ & & & $2(9.1)$ & & \\
\hline Duration of epilepsy (years;months) & & $5 ; 4$ & $(0 ; 9-16 ; 0)$ & & $6 ; 2$ & $(0 ; 7-15 ; 5)$ \\
\hline \multicolumn{7}{|l|}{ Total IQ } \\
\hline$<50$ & $10(38.5)$ & & & $11(50.0)$ & & \\
\hline $50-69$ & $3(11.5)$ & & & $4(18.2)$ & & \\
\hline $70-99$ & $11(42.3)$ & & & $5(22.7)$ & & \\
\hline$\geq 100$ & $2(7.7)$ & & & $2(9.1)$ & & \\
\hline \multicolumn{7}{|l|}{ AEDs } \\
\hline Before KD & & 5.5 & $(3-9)$ & & 5.6 & $(3-9)$ \\
\hline At KD initiation & & 2.4 & $(1-4)$ & & 2.4 & $(0-5)$ \\
\hline \multicolumn{7}{|l|}{ VNS treatment } \\
\hline On & $0(0.0)$ & & & $0(0.0)$ & & \\
\hline Off & $1(3.8)$ & & & $1(4.5)$ & & \\
\hline Epilepsy surgery (callosotomy) & $1(3.8)$ & & & $0(0.0)$ & & \\
\hline \multicolumn{7}{|l|}{ Seizure Frequency at KD initiation } \\
\hline Daily seizures & $10(38.6)$ & & & $3(13.6)$ & & \\
\hline Almost daily seizures & $5(19.2)$ & & & $10(45.5)$ & & \\
\hline$\geq 1$ seizure a week & $7(26.9)$ & & & $6(27.3)$ & & \\
\hline$\geq 1$ seizure a month & $3(11.5)$ & & & $3(13.6)$ & & \\
\hline$<1$ seizure a month & $1(3.8)$ & & & $0(0.0)$ & & \\
\hline Seizure types & & 2.31 & $(1-5)$ & & 2.05 & $(1-4)$ \\
\hline \multicolumn{7}{|l|}{ No changes in AED dose } \\
\hline During baseline & $23(88.5)$ & & & $18(81.8)$ & & \\
\hline At 4 months & $20(87)$ & & & $14(73.7)$ & & \\
\hline \multicolumn{7}{|l|}{ Syndrome classification } \\
\hline West syndrome & $3(11.5)$ & & & $2(9.1)$ & & \\
\hline Lennox-Gastaut syndrome & $1(3.8)$ & & & $0(0.0)$ & & \\
\hline Doose syndrome & $3(11.5)$ & & & $2(9.1)$ & & \\
\hline Dravet syndrome & $1(3.8)$ & & & $0(0.0)$ & & \\
\hline Childhood absence epilepsy & $1(3.8)$ & & & $0(0.0)$ & & \\
\hline Epilepsy with myoclonic absences & $1(3.8)$ & & & $0(0.0)$ & & \\
\hline Generalized epilepsies & $4(15.4)$ & & & $6(27.2)$ & & \\
\hline Localization-related epilepsies & $12(46.4)$ & & & $12(54.6)$ & & \\
\hline \multicolumn{7}{|l|}{ Etiology } \\
\hline Genetic $^{1}$ & $9(34.6)$ & & & $1(4.5)$ & & \\
\hline Structural $^{2}$ & $2(7.7)$ & & & $10(45.5)$ & & \\
\hline Unknown & $15(57.7)$ & & & $11(50.0)$ & & \\
\hline
\end{tabular}


Table 6.1 (continued)

\begin{tabular}{|c|c|c|c|c|c|c|}
\hline & \multicolumn{3}{|l|}{ KD N=26 } & \multicolumn{3}{|c|}{ CAU N=22 } \\
\hline & N (\%) & Mean & (Min-max) & N (\%) & Mean & (Min-max) \\
\hline \multicolumn{7}{|l|}{ Diet type } \\
\hline $\mathrm{MCT}$ & $18(69.2)$ & & & / & & \\
\hline Classical & 7 (26.9) & & & / & & \\
\hline Mix & $1(3.9)$ & & & / & & \\
\hline PGT & $6(23.1)$ & & & $5(22.7)$ & & \\
\hline
\end{tabular}

$\mathrm{N}$, number; Min-Max, minimum-maximum; KD, ketogenic diet; CAU, care as usual, VNS, vagus nerve stimulator; AED, antiepileptic drug; PGT, percutaneous gastrostomy tube. ${ }^{1}$ Genetic: KD: Dravet $(N=1)$, trisomia $13(\mathrm{~N}=1), 1 \mathrm{p} 36$ microdeletion $(\mathrm{N}=1), 16 \mathrm{p} 13.11$ deletion $(\mathrm{N}=1)$, duplication 9q34.11 (N=1), KCNT1 gen mutation ( $\mathrm{N}=1)$, CDKL5 $(\mathrm{N}=1)$, CASK gen mutation $(\mathrm{N}=2)$, CAU: translocation chrom 1 and $11(\mathrm{~N}=1)$. ${ }^{2}$ Structural: KD: severe perinatal asfyxia with $M R I$ abnormalities $(N=1)$, perinatal intracranial bleeding $(N=1)$ CAU: severe perinatal asfyxia with MRI abnormalities $(\mathrm{N}=3)$, severe postnatal hypoglycemia $(\mathrm{N}=1)$ cerebral infarction $(\mathrm{N}=1)$, pneumococcal meningitis $(\mathrm{N}=1)$, hemiatrofia cerebri $(\mathrm{N}=1)$, lissencephaly $(\mathrm{N}=1)$, delayed myelinisation $(N=1)$, tuberous sclerosis complex $(N=1)$.

\section{Seizure frequency}

Seizure frequency reduction for the KD and the CAU group is presented in Table 6.2.

Values at 6 weeks were available for all patients. At 4 months, values for three patients in the CAU group and three in the KD group were missing.

Table 6.2 Comparison of seizure frequency reduction in an intention-to-treat analysis.

\begin{tabular}{|c|c|c|c|c|c|c|}
\hline & & $\begin{array}{l}1 \text { Month } \\
\mathrm{N}(\%)\end{array}$ & $\begin{array}{l}6 \text { Weeks } \\
\mathrm{N}(\%)\end{array}$ & $\begin{array}{l}2 \text { Months } \\
\mathrm{N}(\%)\end{array}$ & $\begin{array}{l}3 \text { Months } \\
\mathrm{N}(\%)\end{array}$ & $\begin{array}{l}4 \text { Months } \\
\mathrm{N}(\%)\end{array}$ \\
\hline \multirow[t]{5}{*}{ KD } & ITT & 26 & 26 & 26 & 26 & 26 \\
\hline & Responders & $9(34.6)$ & 7 (26.9) & $10(38.5)$ & $13(50.0)$ & $13(50.0)$ \\
\hline & Seizure-free & $2(7.7)$ & $2(7.7)$ & $2(7.7)$ & $1(3.8)$ & $3(11.5)$ \\
\hline & $>90 \%$ & $0(0.0)$ & $0(0.0)$ & $2(7.7)$ & $4(15.4)$ & $3(11.5)$ \\
\hline & $>50 \%$ & 7 (26.9) & $5(19.2)$ & $6(23.1)$ & $8(30.8)$ & $7(27.0)$ \\
\hline \multirow[t]{5}{*}{ CAU } & ITT & 22 & 22 & 22 & 22 & 22 \\
\hline & Responders & $5(22.7)$ & $4(18.2)$ & $5(22.7)$ & $5(22.7)$ & $4(18.2)$ \\
\hline & Seizure-free & $1(4.5)$ & $0(0.0)$ & $2(9.1)$ & $1(4.5)$ & $2(9.2)$ \\
\hline & $>90 \%$ & $1(4.5)$ & $0(0.0)$ & $0(0.0)$ & $0(0.0)$ & $1(4.5)$ \\
\hline & $>50 \%$ & $3(13.7)$ & $4(18.2)$ & $3(13.6)$ & $4(18.2)$ & $1(4.5)$ \\
\hline
\end{tabular}

ITT, intention-to-treat; $\mathrm{N}$, number; KD, ketogenic diet; CAU: care as usual.

In an ITT analysis, 13 patients (50\%) treated with the KD for 4 months were responders, three of whom were seizure-free and another three patients had $>90 \%$ seizure reduction. Four patients (18.2\%) of the CAU group were responders; two of them were seizure-free and one had $>90 \%$ seizure reduction.

The percentage of seizures after 4 months, compared to baseline, is presented in Table 6.3. Two extreme outliers in the KD group, who had an increase in seizure frequency exceeding $1000 \%$ due to the increase in number of minor seizures, were removed. Nevertheless, there was a statistically significant difference between the 
proportion of responders versus non-responders, even without removing these extreme outliers $(p=0.022)$.

In both groups, patients were using a mean of 2.4 AEDs at baseline, at the 6-week visit and at the 4-month end-point.

Table 6.3 Comparison of seizures as a percentage of baseline after 4 months.

\begin{tabular}{lccc}
\hline & $\mathrm{KD}$ & $\mathrm{CAU}$ & $\mathrm{p}$-value \\
& $\mathrm{N}=26$ & $\mathrm{~N}=22$ & \\
\hline Mean percentage of baseline & $56 \%$ & $99 \%$ & 0,024 \\
seizures after 4 months (95\% Cl) & $(36-76 \%)$ & $(65-133 \%)$ & \\
Median percentage of baseline & $47 \%$ & $87 \%$ & 0,039 \\
seizures after 4 months (SD, IQR) & $(47,13-74 \%)$ & $(77,58-127 \%)$ & \\
\hline
\end{tabular}

$\mathrm{IQR}$, interquartile range; KD, ketogenic diet; CAU, care as usual.

\section{Seizure severity}

Table 6.4 presents the number of patients with and without an improvement in the NHS3 score compared to baseline.

At 6 weeks, 3 times as many patients using the KD had a relevant decrease in seizure severity score of all seizure types combined, compared with the patients in the CAU group. At 4 months' treatment, there were twice as many patients in the KD group reporting a relevant decrease in seizure severity score. Differences between the two groups were statistically significant at 6 weeks ( $p=0.006$ ); at 4 months, a trend could be detected $(p=0.070)$. Changes in seizure severity were, except for one child of the KD group, related to the most severe seizure type.

Table 6.4 NHS3 score changes on total seizures at six weeks and four months.

\begin{tabular}{lllllllr}
\hline & & \multicolumn{2}{c}{6 weeks } & \multicolumn{3}{c}{4 months } \\
& NHS3 score & KD & CAU & & \multicolumn{1}{c}{ KD } & \multicolumn{1}{l}{ CAU } \\
\hline Total & & N (\%) & N (\%) & p-value & N (\%) & N (\%) & p-value \\
seizures & & & & & & & \\
Score & Improvement & $15(57.7)$ & $4(18.2)$ & 0.006 & $15(65.2)$ & $7(36.8)$ & 0.070 \\
& No improvement & $11(42.3)$ & $18(81.8)$ & 0.006 & $8(34.8)$ & $12(63.2)$ & 0.070 \\
\hline
\end{tabular}

$\mathrm{N}$, number; KD, ketogenic diet; $\mathrm{CAU}$, care as usual.

\section{Side-effects}

\section{SIDAED}

At baseline, there was no statistically significant difference between the patients treated with the KD and the patients of the CAU group regarding each of the nine different domains of side-effects and the total SIDEAD scores.

Total scores showed no statistically significant differences between groups at 6 weeks nor at 4 months. There was, however, a statistically significant difference in gastrointestinal symptoms, where patients treated with the KD having a higher score at 
6 weeks $(p=0.015)$ and at 4 months $(p=0.021)$. The mean value of gastro-intestinal symptoms in the KD group increased from 3.08 at baseline, to 4.08 after 6 weeks and declined to 3.14 after 4 months of treatment.

\section{Kidney stones}

In one child treated with the KD for 6 weeks, abdominal echography was performed because of asymptomatic microscopic hematuria but revealed no abnormalities.

\section{ECG}

No ECG abnormalities appeared, in particular no prolonged QT interval (QTC) time was present.

\section{Anthropometry}

Anthropometric values of 21 children in the intervention group and 17 children in the control group were available.

One child treated with the KD showed a clinically relevant decrease in height, and in another child, there was relevant weight reduction after 4 months on the KD. In the control group, one child had clinically relevant weight loss.

\section{Lipid profile}

Fasting values for total cholesterol, LDL cholesterol and triglycerides were available for $22 \mathrm{KD}$ patients and $17 \mathrm{CAU}$ patients at baseline, and for 16 and 11 patients, respectively, at both 6-week and 4-month visits.

At group level, only the mean value for total cholesterol at 6 weeks' treatment with the KD (5.01 mmol/l (SD 1.15)) was statistically significantly higher $(p=0.03$, mean difference $=0.7$ ) compared to the value of the children in the CAU group (4.31 (SD 0.27)).

\section{Ketosis}

In the KD group, there was a correlation between the mean value of BHB during the first 6 weeks of treatment $(2.2 \mathrm{mmol}$; min-max: 0.5-6.22) and the percentage seizure change at 6 weeks $(p=0.006)$. No other correlations were found.

\section{Discussion}

The goal of our study was to assess the tolerability of the KD and its efficacy on seizure frequency and severity in children and adolescents with refractory epilepsy during a 4-month study period. 
Significantly more patients treated with the KD had a seizure reduction of at least $50 \%$ and a relevant reduction in seizure severity.

Our results are in line with the RCT of Neal et al. and Sharma et al. ${ }^{4,5}$ although the responder rates in the RCT of Neal are somewhat lower (38\% KD vs 6\% CAU) than in our study (50\% KD and $18 \%$ CAU).

Our higher response ratio can be explained by our study design which allowed patients who did not attend the first visit at 6 weeks to be replaced. The response rates in the RCT of Sharma et al. using the MAD are comparable (52\% vs. $11.5 \%$ ). As far as we are aware no RCT comparing MAD and MCT or classical KD had previously been carried out. Miranda et al. compared MAD with a historical cohort treated with the classical KD and found it to be similarly effective. ${ }^{11}$ In our study, two patients in the CAU group spontaneously became seizure-free, compared with none in the previous RCTs. We do not have an explanation other than the natural course of the epilepsy. Furthermore, in our study, two patients in the KD group had a dramatic increase in seizure frequency due to the increase in minor seizures.

The difference between the proportion of responders versus non-responders was statistically significant. The mean seizure frequency at 4 months expressed as a percentage of the baseline was, however, only statistically significantly lower in the KD group compared with CAU after removal of these two extreme outliers. In the other RCTs, the mean seizure frequency at 3 months was significantly less in the diet group; in Neal et al. $62.0 \%$ in the KD group versus $136.9 \%$ in the controls $(p<0.0001)$, and $112.9 \%$ after removal of three extreme outliers in the control group and in Sharma et al. $59.0 \%$ in the KD group versus $95.5 \%$ in the controls $(p<0.003)$.

It is not common to use changes in seizure severity as an outcome parameter in trials. Hallböök et al. described a statistically significant decrease of seizure severity after 3 months KD compared with baseline measured as the mean of the NHS3 value. ${ }^{12}$ In our study, the proportion of patients with a relevant decrease of the seizure severity score was three-fold higher in the KD group at 6 weeks and two-fold higher at 4 months. It is an interesting finding that despite an extreme increase in seizure frequency, patients continue the KD. Decrease of seizure severity is apparently a major factor.

Side-effects using SIDAED showed only a statistically significantly higher score in the KD group in the domain of gastro-intestinal symptoms. The mean value of gastrointestinal symptoms in the KD group at 4 months almost approached the baseline level, although this value was obviously increased at 6 weeks. Fine-tuning the diet can reduce patients' symptoms. The Cochrane review summarized that all studies recorded a range of side-effects, the most prevalent being gastro-intestinal effects in $30 \%$ of patients. ${ }^{3}$

Our results on height and weight are in line with previous literature. In Neal et al., height $z$ scores showed no change at 3 months' treatment but decreased significantly by 6 and 12 months. Weight z scores decreased significantly between baseline and 3, 
6 and 12 months' treatment. ${ }^{13}$ Nordli et al. found adequate height and weight in $96.4 \%$ of infants after 3 months KD. ${ }^{14}$

More clinically severe side-effects reported in the literature are kidney stones. ${ }^{15-17}$ In contrast, no patient in our study developed kidney stones.

Best et al. published the first 3 patients with prolonged QTc while using the KD. ${ }^{18}$

In our study, no abnormalities were found, which is in line with more recent literature. $^{19,20}$

Lipid profile showed only a significant increase of total cholesterol at 6 weeks.

Nizamuddin described an improvement of hypercholesterolemia in approximately half of the patients even without interventions. ${ }^{21}$

There was a statistically significant correlation between being a responder at 6 weeks and the mean value of BHB measured in blood during the first 6 weeks of treatment.

Neal et al. described a significant correlation between serum BHB (and serum acetoacetate) measured at clinical appointments and seizure control at 3 months but not at 6 and 12 months. $^{2}$

This finding is in line with our previous report describing a statistically significant correlation between the single value of BHB at 3 and 6 months' treatment and seizure reduction, but no correlation with ketones in urine. ${ }^{22}$ It seems better to monitor the KD with $\mathrm{BHB}$ measurements than with urinary ketoses. The relationship between seizure control and ketosis is, however, still unclear. ${ }^{4}$

One of the limitations of this open label study is the short study period. Furthermore, we aimed to reach a stable AED dose; this was not always possible due to the complexity of the patients. The percentage of patients with no changes in AED was, however, comparable in both groups. Kverneland et al. described recently a reduction by $35 \%$ of the average serum concentration of AEDs in four adult patients after 12 weeks on $\mathrm{MAD}^{23}$.

The use of seizure diaries in clinical research and practice is accepted and as yet no alternative is available, especially for long term follow-up. The act of self-recording may, however, in itself influence the observation, by causing the subject to be more vigilant about seizures after changing treatment. Subjects may be non-compliant with diary maintenance or may record 'false positive' events that are not seizures. ${ }^{24}$ The use of parental or carer seizure records may well miss some nocturnal or subtle seizures such as myoclonic or absence seizures. ${ }^{4,5}$

In conclusion, this trial provides class I evidence that the KD is an effective therapy compared with CAU, both with regard to seizure frequency and severity, in children and adolescents with refractory epilepsy. Most frequently reported side-effect are gastro-intestinal symptoms that can largely be reduced by fine-tuning the diet. 


\section{References}

1. Kossoff EH, Zupec-Kania BA, Amark PE, et al. Optimal clinical management of children receiving the ketogenic diet: recommendations of the International Ketogenic Diet Study Group. Epilepsia 2009;50:304-17.

2. Neal EG, Chaffe H, Schwartz $\mathrm{RH}$, et al. A randomized trial of classical and medium-chain triglyceride ketogenic diets in the treatment of childhood epilepsy. Epilepsia 2009;50:1109-17.

3. Levy RG, Cooper PN, Giri P. Ketogenic diet and other dietary treatments for epilepsy. Cochrane Database Syst Rev 2012;3:CD001903.

4. Neal EG, Chaffe H, Schwartz RH, et al. The ketogenic diet for the treatment of childhood epilepsy: a randomised controlled trial. Lancet Neurol 2008;7:500-6.

5. Sharma S, Sankhyan N, Gulati S, Agarwala A. Use of the modified Atkins diet for treatment of refractory childhood epilepsy: a randomized controlled trial. Epilepsia 2013;54:481-6.

6. de Kinderen RJ, Lambrechts DA, Postulart D, et al. Research into the (Cost-) effectiveness of the ketogenic diet among children and adolescents with intractable epilepsy: design of a randomized controlled trial. BMC Neurol 2011;11:10.

7. Kwan P, Brodie MJ. Early identification of refractory epilepsy. N Engl J Med 2000;342:314-9.

8. van den Hurk TAM, van der Louw EJTM. Dieetbehandelingsrichtlijn ketogeen dieet voor kinderen (018 jaar) met refractaire epilepsie. Evidence-based handleiding voor een multidisciplinaire behandeling. Utrecht: UMC Utrecht, 2008.

9. O'Donoghue MF, Duncan JS, Sander JW. The National Hospital Seizure Severity Scale: a further development of the Chalfont Seizure Severity Scale. Epilepsia 1996;37:563-71.

10. Uijl SG, Uiterwaal CS, Aldenkamp AP, et al. A cross-sectional study of subjective complaints in patients with epilepsy who seem to be well-controlled with anti-epileptic drugs. Seizure 2006;15:242-8.

11. Miranda MJ, Mortensen M, Povlsen JH, Nielsen H, Beniczky S. Danish study of a modified Atkins diet for medically intractable epilepsy in children: can we achieve the same results as with the classical ketogenic diet? Seizure 2011;20:151-5.

12. Hallbook T, Lundgren J, Rosen I. Ketogenic diet improves sleep quality in children with therapyresistant epilepsy. Epilepsia 2007;48:59-65.

13. Neal EG, Chaffe HM, Edwards N, Lawson MS, Schwartz RH, Cross JH. Growth of children on classical and medium-chain triglyceride ketogenic diets. Pediatrics 2008;122:e334-40.

14. Nordli DR, Jr., Kuroda MM, Carroll J, et al. Experience with the ketogenic diet in infants. Pediatrics 2001;108:129-33.

15. Sampath A, Kossoff EH, Furth SL, Pyzik PL, Vining EP. Kidney stones and the ketogenic diet: risk factors and prevention. J Child Neurol 2007;22:375-8.

16. Paul E, Conant KD, Dunne IE, et al. Urolithiasis on the ketogenic diet with concurrent topiramate or zonisamide therapy. Epilepsy Res 2010;90:151-6.

17. McNally MA, Pyzik PL, Rubenstein JE, Hamdy RF, Kossoff EH. Empiric use of potassium citrate reduces kidney-stone incidence with the ketogenic diet. Pediatrics 2009;124:e300-4.

18. Best TH, Franz DN, Gilbert DL, Nelson DP, Epstein MR. Cardiac complications in pediatric patients on the ketogenic diet. Neurology 2000;54:2328-30.

19. Doksoz O, Guzel O, Yilmaz U, Isguder R, Celegen K, Mese T. Dispersion durations of P-wave and QT interval in children treated with a ketogenic diet. Pediatr Neurol 2014;50:343-6.

20. Sharma S, Gulati S. The ketogenic diet and the QT interval. J Clin Neurosci 2012;19:181-2.

21. Nizamuddin J, Turner Z, Rubenstein JE, Pyzik PL, Kossoff EH. Management and risk factors for dyslipidemia with the ketogenic diet. J Child Neurol 2008;23:758-61.

22. van Delft R, Lambrechts D, Verschuure P, Hulsman J, Majoie M. Blood beta-hydroxybutyrate correlates better with seizure reduction due to ketogenic diet than do ketones in the urine. Seizure 2010;19:36-9.

23. Kverneland M, Tauboll M, Selmer KK, Iversen PO, Nakke KO. Modified Atkins diet may reduce serum concentrations of antiepileptic drugs. Acta Neurol Scand 2015:131:187-90.

24. Fisher RS, Blum DE, DiVentura B, et al. Seizure diaries for clinical research and practice: limitations and future prospects. Epilepsy Behav 2012;24:304-10. 


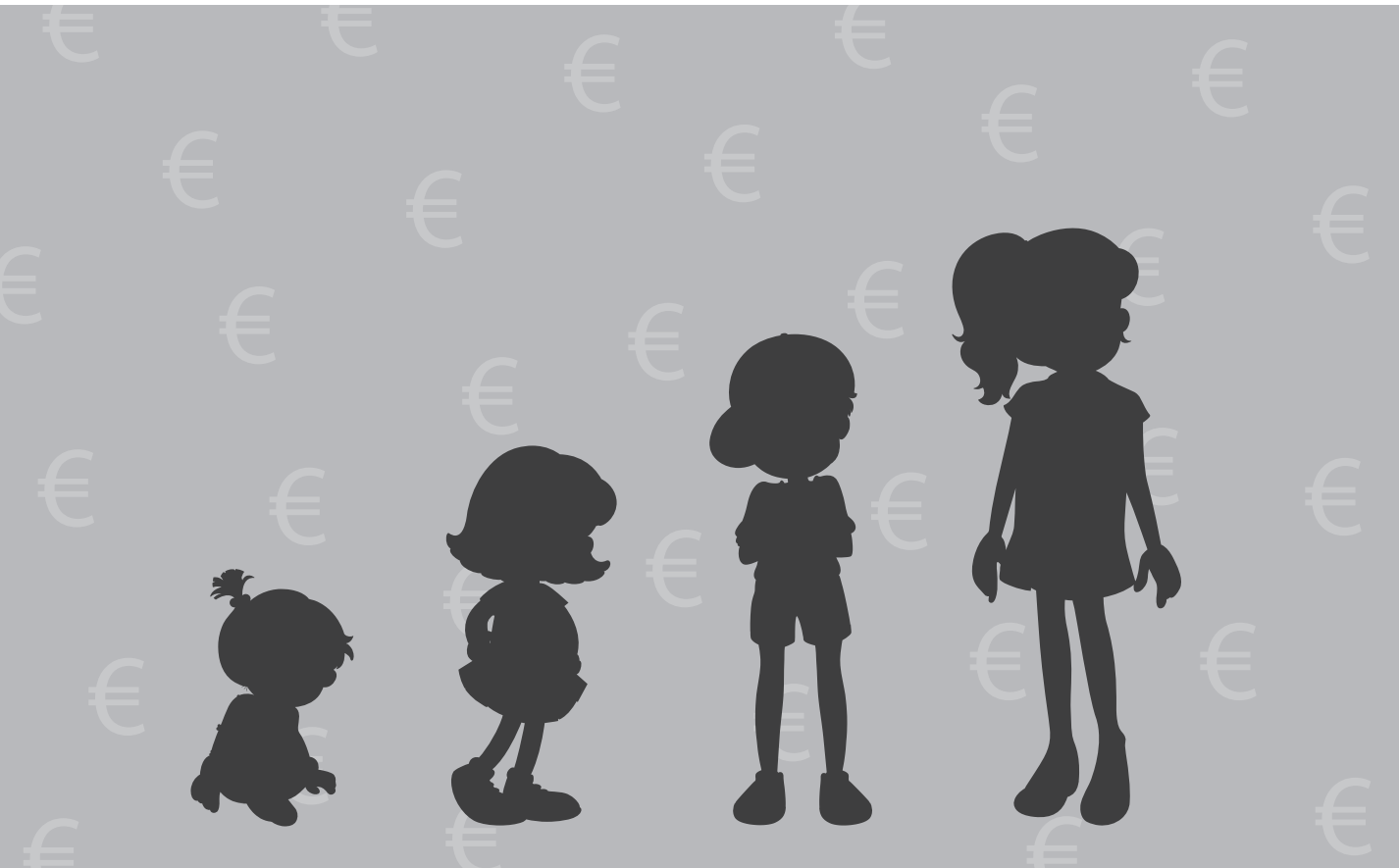




\section{Chapter 7}

An economic evaluation of the ketogenic diet versus care as usual in children and adolescents with intractable epilepsy: an interim analysis 


\section{Abstract}

\section{Objectives}

To gain insight in the cost-effectiveness of the ketogenic (KD) diet compared with care as usual (CAU) in children and adolescents with intractable epilepsy, we conducted an economic evaluation from a societal perspective, alongside a randomized controlled trial.

\section{Methods}

Participants from a tertiary epilepsy center were randomized into KD (intervention) group or CAU (control) group. Seizure frequency, quality adjusted life years (QALYs), health care costs, production losses of parents and patient, and family costs were assessed at baseline and during a 4-month study period and compared between the intervention and control group. The incremental cost-effectiveness ratios (ICERs) (i.e. cost per QALY and cost per responder), and cost-effectiveness acceptability curves (CEACs) were calculated and presented.

\section{Results}

In total, 48 children were included in the analyses of this study (26 KD group). At 4 months, $50 \%$ of the participants in the KD group had a seizure reduction $\geq 50 \%$ from baseline, compared with 18.2 of the participants in the CAU group. The mean costs per patient in the CAU group were $€ 15,245$ compared to $€ 20,986$ per patient in the KD group, resulting in an ICER of $€ 18,044$ per responder. We failed, however, to measure any benefits in terms of QALYS and therefore, the cost per QALY rise high above any acceptable ceiling ratio. It might be that the quality of life instruments used in this study were not sufficiently sensitive to detect changes, or it might be that being a clinical responder is not sufficient to improve a patient's quality of life. Univariate and multivariate sensitivity analyses and nonparametric bootstrapping were performed, and demonstrated the robustness of our results.

\section{Significance}

The results show that the KD reduces seizure frequency. The study did not find any improvements in quality of life and, therefore, unfavorable cost per QALY ratio's resulted. 


\section{Introduction}

Epilepsy is a major, cost-intensive and global health problem. ${ }^{1}$ In the Netherlands, the prevalence of epilepsy is estimated to be five per 1,000 of the population; at any one time, about 80,000 individuals have epilepsy. ${ }^{2}$ In 2004, the total costs of epilepsy in Europe were $€ 15.5$ billion, indirect costs being the single most dominant cost category (€8.6 billion). ${ }^{3}$ In 2011, in the Netherlands, the direct medical costs were $€ 248$ million, which is $0.3 \%$ of the health care budget. ${ }^{4}$ Although in the majority of patients epilepsy is treatable with antiepileptic drugs (AEDs), about $30 \%$ of patients have intractable epilepsy. ${ }^{5}$ Patients are diagnosed as having intractable epilepsy when their seizures are not controlled after taking two or more different AEDs. Patients with uncontrolled epilepsy depend heavily on informal care (family and friends), and on health care professionals (neurologists, social workers, psychologists, and so on). Complications due to intractable epilepsy result in frequent hospitalizations and many of these patients are institutionalized. Living with uncontrolled seizures has a negative impact on the Quality of Life (QOL) of the child and his or her surroundings.

Patients with intractable epilepsy after failure of AEDs and/or resective surgery can potentially benefit from a ketogenic diet (KD). The KD is a high-fat, low-carbohydrate diet that imitates the metabolic state of fasting while maintaining a normal number of calories. Body energy requirements while on a KD are met by lipolysis and ß-oxidation of fatty acids rather than by the breakdown of carbohydrates. The exact anticonvulsant mechanism of action of a KD has not yet been elucidated. ${ }^{6}$ Currently, the scientific and clinical attention directed toward the role of the KD is negligible. ${ }^{7}$ This means that the KD is often overlooked and underutilized as a treatment option for children with intractable epilepsy. An important reason for this is that relatively few children and their parents can comply with the stringent diet. Physicians are, therefore, often reluctant to initiate the diet. The beneficial effect of a KD has been studied in multiple observational studies, ${ }^{8-15}$ reviews, ${ }^{16-19}$ and in two randomized controlled trials. ${ }^{20,21}$ However, none of these studies has researched the costeffectiveness of the KD. Because resources are scarce, in current health care decisionmaking, we need to prove that the effects of the KD are worth the extra costs, to allow decisions to be evidence-based. ${ }^{22-23}$ Economic evaluation is a method for examining this trade-off between costs and effects of comparative treatments. Full economic evaluations are necessary to inform the decision makers. Therefore, this trial-based economic evaluation researches the cost-utility and cost-effectiveness of the KD compared with care as usual (CAU) in children and adolescents with intractable epilepsy from a societal perspective. 


\section{Methods}

The economic evaluation was executed alongside a randomized controlled trial (RCT); details of the trial design can be found elsewhere. ${ }^{24}$ In short, between July 2010 and September 2014, patients were randomized to either the KD or to CAU after a 1-month baseline period. Patients randomized into the KD group were followed during the 4-month comparative study period and a follow-up period of 12 months. Patients randomized into the CAU group were followed only during the 4-month study period. The timeline of the study is graphically presented in Figure 7.1. This interim analysis reports the cost-effectiveness of the comparative period of the trial, that is, the 4-month study period where direct comparison between KD and CAU is possible. This study has been approved by the ethics committee of the Academic Medical Center Utrecht, the Netherlands.

The economic evaluation was performed from a societal perspective and consisted of a cost-utility analysis with quality adjusted life years (QALYs) as primary outcome and a cost-effectiveness analysis with treatment responder ( $\geq 50 \%$ seizure reduction) as the primary outcome.

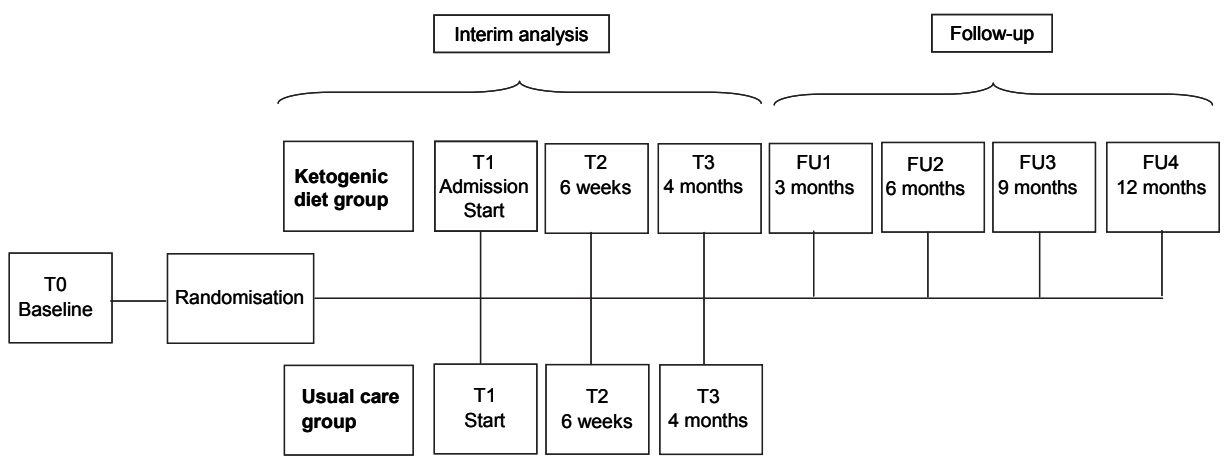

Figure 7.1 Timeline of the study.

\section{Study population and sample size}

Patient were children and adolescents with intractable epilepsy. Patients were eligible to participate if they met the following criteria: aged between 1 and 18 years; having uncontrolled seizures after trying two or more AEDs; not eligible for epilepsy surgery; no fatty acid oxidation disorders and related diseases; no diabetes and hyperinsulinism; no long QT-time syndrome; no hypercholesterolemia or hypertriglyceridemia; no severe liver, kidney or pancreas diseases; no renal tubular acidosis; no severe behavioral disorder; no malnutrition; no treatment with topiramate or acetazolamide and no positive family history or other risk factors for kidney stones or 
acidosis. Eligible candidates and their parents were referred by their attending physician to the multidisciplinary KD team at epilepsy center Kempenhaeghe in the Netherlands, where they received detailed information about the study before giving their consent.

This study was performed alongside a RCT where seizure frequency was the primary outcome. Based on a minimum detectable difference in success rate of $35 \%$ between the $\mathrm{KD}$ group and $\mathrm{CAU}$, and assuming that alpha $=5 \%$ and power $=80 \%$, we needed 22 children for each group. ${ }^{24}$

\section{Interventions}

\section{Ketogenic diet}

Patients assigned to the KD group were admitted to the tertiary epilepsy center for 5 days, while KD was introduced by a dietician. The neurologist, pediatrician and epilepsy nurse visited the child during the 5-day admission and ketosis was assessed daily in urine (if toilet-trained) and three times in blood by means of a finger puncture. The AEDs used by the children and adolescents at the time of inclusion in the study were continued without changes during the study period (except when medically indicated). The dietician decided together with the parents whether the classical diet, the medium-chain triglyceride (MCT) diet, or a mixture of both diets was introduced.

Children who were tube-fed were also treated with KD. The diet was then adjusted to a fluid version.

After the 5-day admission, weekly telephone meetings were planned between parents and the epilepsy nurse, and between parents and the dietician. Ketosis was checked daily by the parents in urine or three times a week in blood via a finger puncture. Furthermore, time points T2 and T3 consisted of a visit to the neurologist, pediatrician, dietician and epilepsy nurse. At T3, blood and urine samples were taken from the patients in the intervention group. No other protocol-driven care was delivered.

\section{Care as usual}

Patients randomized to CAU continued to take their AEDs. The controls were treated and monitored according to good clinical practice. Patients randomized into CAU were seen at time points T2 and T3 by a neurologist, pediatrician and epilepsy nurse. Weekly telephone meetings were scheduled between parents and the epilepsy nurse. No other protocol-driven care was delivered. 


\section{Outcome measures}

Seizure frequency

A daily seizure calendar was used to record seizures continuously. Types of seizures were described and labeled in accordance with the International League Against Epilepsy (ILAE) classification. A patient was labeled as a responder when he or she had a mean reduction in seizures of $50 \%$ or more during the last month of the study period (month 4) compared with baseline. Based on previous literature, a cut-off point of $\geq 50 \%$ seizure reduction was considered to be a realistic clinical target for patients with intractable epilepsy.

\section{Utilities}

QOL of the children and their parents was measured using the EuroQol-Youth (EQ-5D$\mathrm{Y})$ version for children aged 8 years and older, and the EuroQol (EQ-5D), respectively. The EQ-5D system comprises the following five dimensions: mobility, self-care, usual activities, pain/discomfort and anxiety/depression. Each dimension has three levels: no problems, some problems, and extreme problems. ${ }^{25,26}$ The EQ-5D health states were converted to a single summary index between 0 and 1 (utility) by applying the Dutch utility-tariff. ${ }^{27}$ In addition, QOL was assessed by the TAPQOL (TNO-AZL Preschool Children's Quality of Life) for children aged between 1 and 5 years (parent proxy) and the TACQOL (TNO-AZL Children's Quality of Life) for children aged between 6 and 16 years (parent proxy). ${ }^{28,29}$ The TAPQOL has nine domains, all ranging between 0 and 100. The TACQOL has seven domains, two of which have scores ranging between 0 and 32 and two domains between 0 and 16. All domains were given equal weight; a mean score was calculated and transformed into a QOL-score between 0 and 1. Utility scores from the EQ-5D, EQ-5D-Y, and QOL-scores from the TAPQOL and TACQOL were measured at time points T0 and T3.

QALYs were calculated by means of the "under the curve method", in which the time in a certain health state was multiplied by the utility of this health state. ${ }^{22}$

\section{Costs}

Costs were calculated from a societal perspective; therefore, protocol-driven intervention costs, other health care costs, patient and family costs, and productivity losses of the parent(s) were measured. Costs were expressed in Euros and were converted to the price year 2013 based on the relevant consumer price index. Due to the time horizon of 4 months, no discounting was performed.

Other (epilepsy-related) health care costs and patient and family costs were measured by a monthly cost diary, completed by the patient's parent(s). ${ }^{30}$ Because there are no institutional records tracking information on the type, frequency and magnitude of resource use in the cost sectors "other health care costs" and "patient and family costs", data must be obtained through a self-report "resource use measure", such as 
questionnaires and diaries. In comparison with questionnaires, diaries have been reported to provide information prospectively over a period of time, resulting in minimum recall bias. ${ }^{30}$ Additional costs consisted of parents' productivity losses, based on the hours absent from work due to their child's epilepsy or their own healthrelated problems. Parents' productivity costs were taken into account because parents are (sometimes) forced to be absent from work to provide care for their child or because of their own health problems that may be indirectly caused by their child's disease (e.g., due to increased stress levels).

Costs were estimated using a bottom-up approach, in which information on each element of service used was multiplied by an appropriate standardized unit cost and summed to provide an overall total cost. ${ }^{22}$ Where possible, unit costs were based on standardized costs from the Dutch guidelines for cost research. ${ }^{31}$ Real tariffs or costs were used when no standardized unit costs were available. Cost of medication was calculated using guideline prices. ${ }^{31}$ An addition for prescription charges for prescribed medication was taken into account. When data on medication were diverse, lowest cost prices for the specific medication were used. The cost of informal care was calculated using shadow pricing. For this, the general hourly minimum wages were applied. ${ }^{31}$ Productivity costs were calculated on the basis of the friction cost method (FCM), following the Dutch guidelines. ${ }^{31}$ The FCM is based on the assumption that an organization needs a certain time span (friction period) to replace the absent worker by another worker. The definitive number of days absent from work is then limited to the duration of the friction period, which was determined in the Netherlands to be 23 weeks in $2010 .^{31}$ Unit prices are presented in Table 7.1.

\section{Data analyses}

The economic analyses were carried out on an intention to treat (ITT) protocol. Mean imputation was used to impute missing Quality Adjusted Life Years (QALYs; N=5) and cost data within the treatment group $(\mathrm{N}=1)$. For incomplete cases last observation was carried forward $(n=4)$. Due to the relative low proportion of missing values, less sophisticated methods were used to handle missing data.

Data were analyzed using IBM SPSS Statistics version 22. Baseline differences in costs and utilities between groups were checked with non-parametric bootstrapping, based on 1,000 bootstrap replications, as the data did not comply with the underlying assumptions of parametric tests.

Furthermore, the arithmetic mean and 95\% non-parametric confidence intervals were calculated (1,000 bootstrap replications) for differential costs and QALYs during the study period. 
Table 7.1 Unit prices.

\begin{tabular}{|c|c|}
\hline & $€(2013)$ \\
\hline \multicolumn{2}{|l|}{ Intervention costs (4 months) } \\
\hline KD group & $4,311.81^{a}$ \\
\hline CAU group & $1,548.60^{\mathrm{a}}$ \\
\hline \multicolumn{2}{|l|}{ Diet costs (4 months) } \\
\hline Classical diet & 0 \\
\hline MCT diet & $2,491.20^{\mathrm{a}}$ \\
\hline Tube diet & $3,476.40^{a}$ \\
\hline Mixture & $1,246.60^{a}$ \\
\hline \multicolumn{2}{|l|}{ Ketosis check } \\
\hline Finger puncture device & $21.73^{\mathrm{a}}$ \\
\hline In blood (finger puncture) & $2.02^{\mathrm{a}}$ \\
\hline In urine & $0.14^{\mathrm{a}}$ \\
\hline \multicolumn{2}{|l|}{ Health care costs } \\
\hline GP visit & $30.48^{b}$ \\
\hline Specialist visit & $140.42^{b}$ \\
\hline Epilepsy nurse visit & $109.65^{a}$ \\
\hline Blood sample & $24.77^{\mathrm{a}}$ \\
\hline Physiotherapist visit & $39.19^{b}$ \\
\hline Speech therapist visit & $35.92^{b}$ \\
\hline Dietician visit & $29.39^{b}$ \\
\hline Other paramedic visit & $23.95^{b}$ \\
\hline Psychologist visits & $87.08^{b}$ \\
\hline Psychiatrist visit & $112.12^{b}$ \\
\hline Routine EEG & $273.55^{a}$ \\
\hline 24-hours EEG & $779.65^{\mathrm{a}}$ \\
\hline MRI & $179.84^{\mathrm{a}}$ \\
\hline General hospital (day) & $473.50^{b}$ \\
\hline Academic hospital (day) & $625.89^{b}$ \\
\hline Epilepsy center (day) & $208.25^{a}$ \\
\hline Inpatient disabled care (day) & $208.25^{a}$ \\
\hline Day care & $273.21^{b}$ \\
\hline Home care (hour) & $70.75^{b}$ \\
\hline Social services (hour) & $70.75^{b}$ \\
\hline Prescribed medication & Variable $^{c}$ \\
\hline \multicolumn{2}{|l|}{ Patient and family costs } \\
\hline Informal care (hour) & $13.61^{b}$ \\
\hline Child care & Variable $^{a}$ \\
\hline Housekeeping (hour) & $13.61^{b}$ \\
\hline Medication (ОTC) & Variable $^{c}$ \\
\hline Activities & Variable $^{a}$ \\
\hline Transport (per kilometer) & $0.20^{\mathrm{b}}$ \\
\hline \multicolumn{2}{|l|}{ Other costs } \\
\hline Production losses (hour) & $32.68^{b}$ \\
\hline
\end{tabular}

${ }^{a}$ (Calculated from) respective providers or professional organizations. ${ }^{b}$ Dutch guidelines for costing studies.

${ }^{c}$ Dutch Pharmaceutical Therapeutic Compass.

In addition, bootstrapping with Microsoft Excel 2010 was used to quantify the uncertainty around the incremental cost effectiveness Ratio (ICER) (5,000 bootstrap replications). The ICERs were calculated by dividing the incremental costs by the 
incremental QALYs. The ICER represents the cost of an additional QALY gained and was used to estimate the cost-utility of KD as opposed to CAU. The ICERs were presented on a cost-effectiveness (CE) plane, which is divided into four quadrants. The south-east quadrant represents the instances where the intervention is more effective and less costly than the comparators (dominates), with the opposite situation in the north-west quadrant, representing interventions that are more costly and less effective than comparators (dominated). The north-east quadrant represents interventions that are more costly yet more effective, whereas the south-west quadrant represents less effective and less costly interventions. ${ }^{22} \mathrm{~A}$ treatment is deemed cost-effective when it is dominant or when the costs per QALY are below a specified ceiling ratio. In the Netherlands, an informal ceiling ratio exists between approximately $€ 20,000$ and $€ 80,000$ per QALY, depending on the burden of disease. ${ }^{32}$ The burden of disease can be expressed in disability weights, ranging from 0 (implying no loss of health) to 1 (implying a health loss equivalent to death). The disability weight of severe epilepsy is estimated to be $0.657^{33}$, this corresponds to a threshold of approximately $€ 50,000$ per $Q A L Y,{ }^{32}$ which was taken as a ceiling ratio in this study. To show the probability that a KD is cost-effective, given different ceiling ratios, a cost-effectiveness acceptability curve (CEAC) was constructed. ${ }^{22}$ Furthermore, the number of responding patients ( $\geq 50 \%$ seizure reduction) and the incremental cost per responding patient were calculated.

Univariate and multivariate sensitivity analyses were performed to explore how robust the results were to changes in the assumptions upon which they were based. First, the scenario was calculated in which the economic evaluation was performed from the health care perspective in order to comply with the guidelines of other policy bodies. $^{34}$ Second, we explored the impact on the cost-effectiveness of the KD by varying the cost of the intervention and diet, as these costs are based mainly on the specific protocol of epilepsy center Kempenhaeghe. Other centers initiate the KD during outpatient visits and/or offer only the classical diet, possibly due to reimbursement issues. We therefore included this realistic and policy-relevant sensitivity analysis to provide the reader with information about the costeffectiveness if another protocol, policy or organization of care is followed.

\section{Results}

Between August 2010 and September 2014, a total of 58 patients were included in the study. One patient dropped out of the study before and nine patients dropped out after randomization: six from the control group, due to dissatisfaction regarding the randomization result, and three patients from the KD group, due to spontaneous seizure reduction (one patient) and gastro-intestinal side effects (two patients), leaving 48 patients in the ITT analysis. 
There were no statistical differences between the KD and CAU group at baseline in utilities (both TACQOL/TAPQOL and EQ-5D) or in total health care, patient, and family and productivity losses. Therefore, baseline correction was assumed to be unnecessary. Demographic data and baseline utilities are presented in Table 7.2.

Table 7.2 Demographic characteristics and baseline utilities.

\begin{tabular}{|c|c|c|}
\hline & $\begin{array}{c}\mathrm{KD} N=26 \\
\mathrm{~N}(\%)\end{array}$ & $\begin{array}{c}\text { CAU N=22 } \\
N(\%)\end{array}$ \\
\hline Female & $8(30.8)$ & $13(59.1)$ \\
\hline Age mean (range) & $7.8(2.1-16.5)$ & $8.1(1.1-15.7)$ \\
\hline Age seizure onset (range) & $2.4(0-8)$ & $1.9(0-10)$ \\
\hline Genetic & $9(34.6)$ & $1(4.5)$ \\
\hline Structural & $2(7.7)$ & $10(45.5)$ \\
\hline Unknown & $15(57.7)$ & $11(50.0)$ \\
\hline \multicolumn{3}{|l|}{ Full scale IQ } \\
\hline$<50$ & $10(38.5)$ & $11(50.0)$ \\
\hline $50-69$ & $3(11.5)$ & $4(18.2)$ \\
\hline 70-99 & $11(42.3)$ & $5(22.7)$ \\
\hline$\geq 100$ & $2(7.7)$ & $2(9.1)$ \\
\hline \multicolumn{3}{|c|}{ Behavioral/Cognitive problems } \\
\hline ADHD & $0(0.0)$ & $1(4.5)$ \\
\hline ADD & $1(3.8)$ & $1(4.5)$ \\
\hline Autism & $3(11.5)$ & $5(22.7)$ \\
\hline \multicolumn{3}{|l|}{ Number of AEDs at start study } \\
\hline None & $0(0.0)$ & $1(4.5)$ \\
\hline One & $3(11.5)$ & $4(18.2)$ \\
\hline Two & $12(46.2)$ & $8(36.4)$ \\
\hline Three & $9(34.6)$ & $5(22.7)$ \\
\hline Four & $2(7.7)$ & $2(9.1)$ \\
\hline Five & $0(0.0)$ & $2(9.1)$ \\
\hline \multicolumn{3}{|l|}{ Diet type } \\
\hline $\mathrm{MCT}$ & $18(69.2)$ & - \\
\hline Classical & 7 (26.9) & - \\
\hline Mixture & $1(3.9)$ & - \\
\hline PGT & $6(23.1)$ & $5(22.7)$ \\
\hline \multicolumn{3}{|l|}{ Mean quality of life } \\
\hline TACQOL or TAPQOL & 0.77 & 0.75 \\
\hline EQ-5D-youth ${ }^{\mathrm{a}}$ & 0.53 & 0.64 \\
\hline \multicolumn{3}{|l|}{ Number AEDs tried in the past } \\
\hline Three & $3(11.5)$ & $4(18.2)$ \\
\hline Four & $4(15.4)$ & $5(22.7)$ \\
\hline Five & $9(34.6)$ & $3(13.6)$ \\
\hline $\operatorname{Six}$ & $3(11.5)$ & $3(13.6)$ \\
\hline Seven & $2(7.7)$ & $1(4.5)$ \\
\hline Eight & $4(15.4)$ & $2(9.1)$ \\
\hline Nine & $1(3.8)$ & $4(18.2)$ \\
\hline VNS in the past & $1(3.8)$ & $1(4.5)$ \\
\hline Epilepsy surgery in the past & $1(3.8)$ & $0(0.0)$ \\
\hline
\end{tabular}

KD: Ketogenic diet, CAU: Care as usual, N: Number, AEDs: antiepileptic drugs, ADHD: attention deficit hyperactivity disorder, ADD: attention deficit disorder, VNS: vagus nerve stimulator, MCT: medium-chain triglycerides, PGT: percutaneous gastrostomy tube. ${ }^{a}$ Based on $\mathrm{N}=10 \mathrm{KD}$ group and $\mathrm{N}=11 \mathrm{CAU}$ group. 


\section{Outcomes}

In total, 13 patients from the KD group (50\%) and 4 patients from the CAU group $(18.2 \%)$ were responders ( $\geq 50 \%$ seizure reduction). Measuring utilities with the EQ$5 \mathrm{D}-\mathrm{Y}$ seemed only possible in a minority of patients in our sample. We, therefore, used the QOL-scores of the TAPQOL and TACQOL (age-dependent) to calculate QALYS for the patients. The QALYs of the parents were based on utilities of the EQ-5D. No significant differences were found in patients' QALYs or in parents' QALYs between the two groups (Table 7.3). Total health care costs differed significantly between the two groups due to significantly higher intervention and diet costs in the KD group. No other significant cost differences were found.

\section{ICERs}

The CE plane for the cost-utility analysis from a societal perspective, representing the uncertainty surrounding the cost per QALY ratio, is shown in Figure 7.2A. The KD is more expensive than CAU (resp. $€ 20,986$ and $€ 15,245$ ) and (almost) equally effective in terms of QALYs (resp. 0.253 and 0.250). As a result, most bootstrapped ICERs lie in the northern part of the CE plane ( $40 \%$ north-west and $56 \%$ north-east). Based on the cost-utility analysis, the $K D$ is not a cost-effective treatment option. Figure 7.2B presents the CEAC based on the cost per QALY, showing the probability that the KD is cost-effective at different ceiling ratios. At the ceiling ratio of $€ 50,000$, there is only a $5 \%$ probability of the KD being cost-effective.

The incremental costs (KD: $€ 20,986, C A U: € 15,245)$ divided by the incremental number of responders, taking the difference in group sizes into account (KD: 13/26, CAU: $4 / 22$ ), resulted in an ICER of $€ 18,044$ per responder. The uncertainty analysis of this ICER is presented in the CE plane in Figure 7.3. Most ICERs lie in the north-east quadrant, indicating that the KD is more expensive but also more effective than CAU in children and adolescents with intractable epilepsy.

\section{Sensitivity analyses}

First, performing the analyses from a health care perspective and at a ceiling ratio of $€ 50,000$, results showed that the KD has only $3 \%$ probability of being cost-effective when looking at the cost per QALY (CE planes and CEACs not presented). From a health care perspective, the mean costs per patient in the KD and CAU group are resp. $€ 12,139$ and $€ 7,589$ (see Table 7.3), resulting in an ICER of $€ 14,300$ per responder.

Second, lowering the cost of the intervention by initiating the KD without 5-day admission to the epilepsy center, ignoring the telephone meetings with dietician and/or nurse (in both groups), and simultaneously lowering the cost of the KD by offering solely the classical variant of the diet, the KD has a $32 \%$ probability of being cost-effective at a ceiling ratio of $€ 50,000$ per QALY from a societal perspective (CE planes and CEACs not presented). In this situation, the mean costs per patient in the KD group are $€ 16,241$ compared to $€ 14,478$ in the CAU group, resulting in an ICER of the costs per responder of $€ 5,544$. 


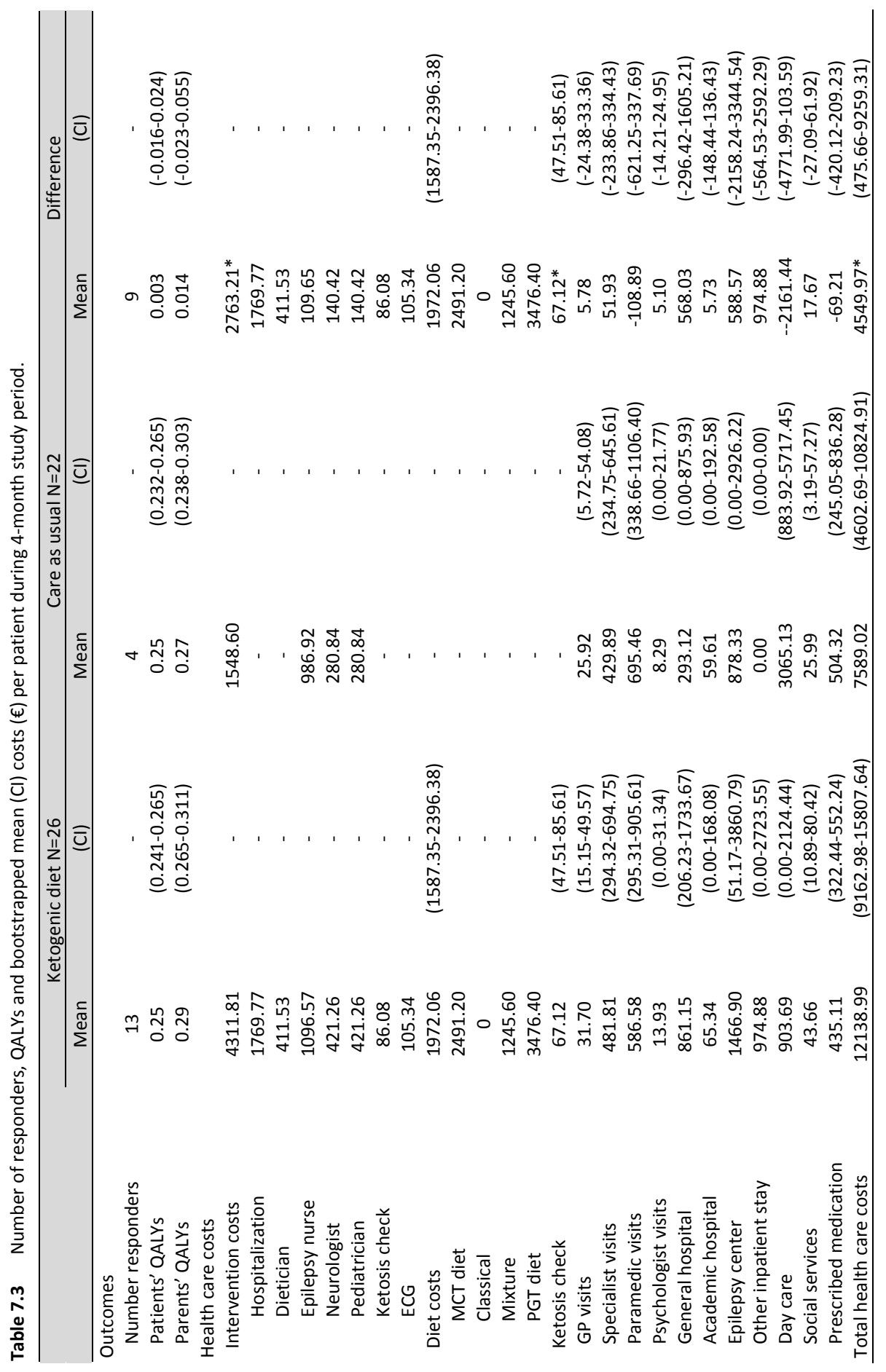




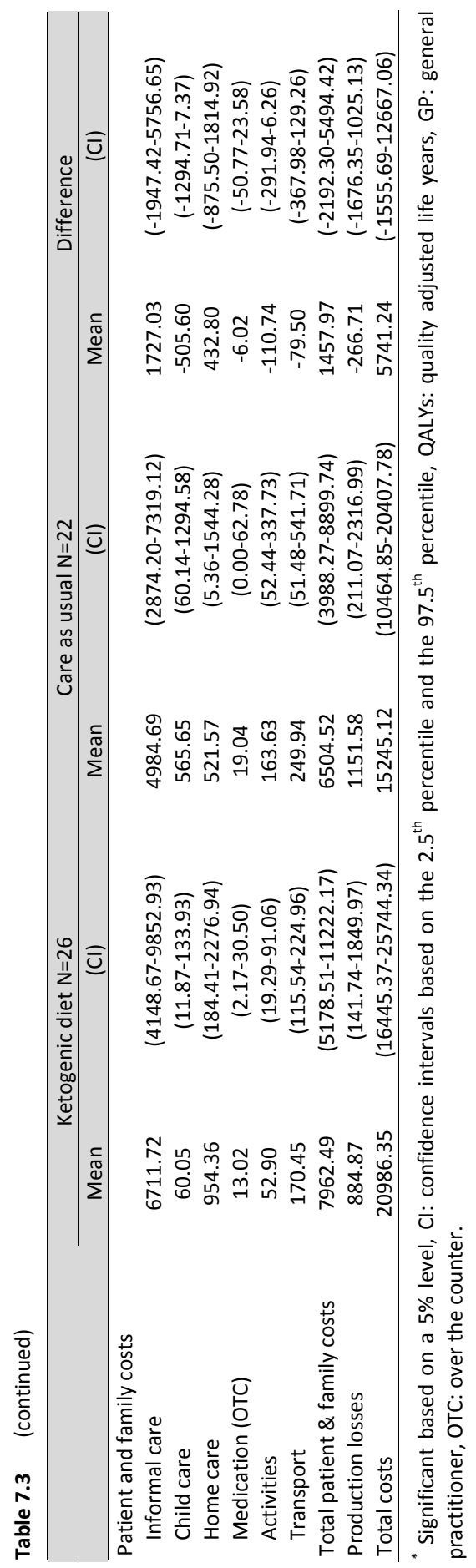



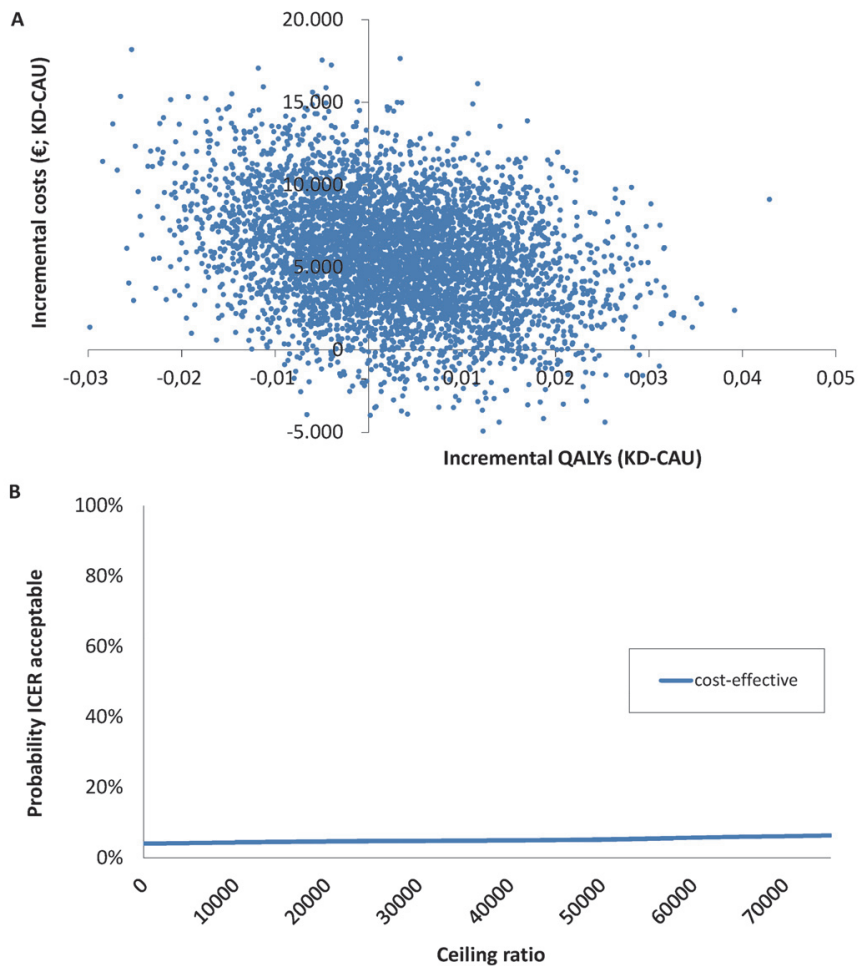

Figure 7.2 (A) Cost-effectiveness plane, cost per QALY; (B) Cost-effectiveness acceptability curve, cost per QALY.

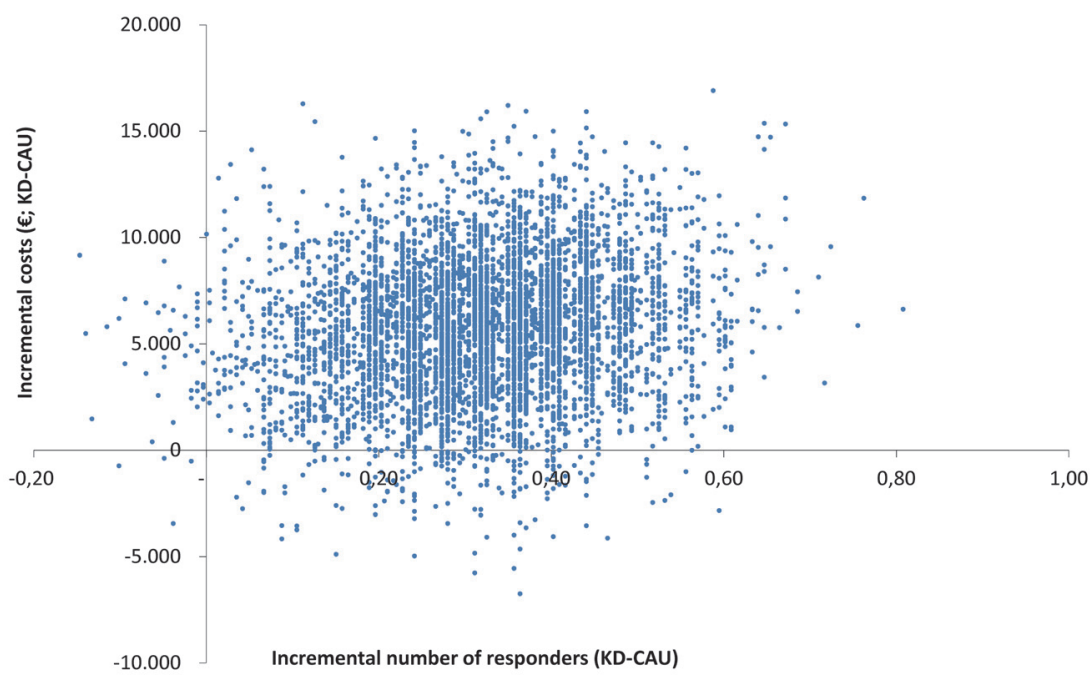

Figure 7.3 Cost-effectiveness plane, cost per responder. 


\section{Discussion}

To our knowledge, this is the first study reporting an economic evaluation comparing KD with CAU in children and adolescents with intractable epilepsy alongside a randomized controlled trial. The economic evaluation was conducted from the societal perspective.

The main results of this study are the following: 1 ) the KD is more effective than CAU in terms of seizure reduction: resp. $50 \%$ and $18.2 \% ; 2$ ) the mean costs per participant were $€ 20,986$ for the KD compared to $€ 15,245$ for CAU, resulting in an ICER of the costs per responder of $€ 18,044$; and 3 ) we did not find any benefits in terms of QALYs and therefore, the cost per QALY rise high above any acceptable ceiling ratio. Hence, this 4-month analysis shows that the KD is not cost-effective when including the QALY as a primary outcome parameter. This apparent lack of effectiveness of the KD in terms of QOL could point towards the KD being an ineffective way of treating patients with epilepsy. This conclusion, however, is too short-sighted and needs some nuance. On the one hand, the question arises whether the QOL instruments used in our study were sufficiently sensitive to measure clinical changes (e.g. being a responder). First, it is likely that a generic instrument such as the EQ-5D-Y is not able to detect patients' clinical improvements on broadly defined (health) domains. Second, half of the patients in our study were children and adolescents with combinations of severe intellectual and physical disabilities which makes measuring QOL a huge challenge. ${ }^{35}$ It is possible that proxies have difficulties taking into account changes in mood and anxiety levels, especially in patients having troubles expressing themselves. On the other hand, being a clinical responder (i.e. seizure reduction of $50 \%$ or more) may not be sufficient to have an impact on a patient's QOL as patients are left with disabling seizures. Total seizure freedom has, however, been reported to be one of the most important factors determining QOL in patients with epilepsy. ${ }^{36}$ Although the KD is effective in reducing seizure frequency, in our patient population with severe intractable epilepsy, it is very unlikely to reach seizure freedom in many patients and hence to produce a major change in QOL. Seizure reduction may not lead directly to improved QOL: however, it is an important outcome measure for comparability purposes, as it is required by European and other health authorities and it has been used as a primary outcome measure in many other trials including KD and VNS. Furthermore, QOL is a comprehensive concept consisting of multiple dimensions. Other factors will influence QOL in patients with intractable epilepsy such as stigma, anxiety, depression and the unpredictability of seizures. This complexity might be one of the reasons why it is so hard to reach a QOL improvement. For future studies, one should keep in mind that perhaps other preference-based QOL instruments are more suitable and/or sensitive in children with epilepsy. For example, the Child Health Utility-9D (CHU-9D) ${ }^{37}$ or Assessment of Quality Of Life-6D (AQOL-6D) ${ }^{38}$, which both became available after the start of our trial, might be a welcome addition in studying QOL in children with epilepsy. 
Our results are sensitive to change, as can been seen in the sensitivity analyses, where we hypothetically decreased the intervention costs, and simultaneously increased the classical diet users from $27 \%$ to $100 \%$. This resulted in an increased probability (32\%) for the KD to be cost-effective at a ceiling ratio of $€ 50,000$ per QALY.

This study has some limitations. The first limitation concerns the utility measurement. We were aiming at calculating the cost per QALY, as this is essential information for policy makers. However, at the start of the study, there was no suitable preferencebased generic QOL instrument available for utility measurements in children that covered the full developmental range between 0 and 18 years. To calculate QALYs, a QOL-score between 0 and 1 was used, which we adapted from the scores of the agedependent TAPQOL and TACQOL. The QOL-scores we used to calculate QALYs, are, therefore, not preference-based utilities and subsequently not comparable with other "real" utilities. In addition, because the TAPQOL and TACQOL are age-dependent measures, our QOL-scores consist of a combination of both measures, limiting the comparability of utility measurement among different age groups. Furthermore, the utilities derived from the EQ-5D-Y measurement are calculated with an adult valuation tariff $^{27}$, which is not recommended by the EuroQol Group.

The second limitation is the small sample size. As is often the case when conducting trial-based economic evaluations, the sample size for this study was based on a clinical outcome measure (i.e., seizure frequency) and not on QOL or costs as the primary outcome. Hence, there is a probability that the sample size was too small to detect changes in patients' QOL between both study arms.

The third limitation is the short time horizon of the study, which can be the reason for our inability to measure change in QALYs or costs. This analysis compares the KD group with the CAU group over a period of 4 months. Although a longer comparative period would be preferable, this was not possible due to ethical considerations. The significant differences in costs were fully related to the intervention and diet costs in the KD group. We did not find any reduction in health care costs, patient and family costs or in productivity losses. Other inter-sectoral costs, such as educational costs, were not taken into account, as the Dutch guidelines do not include guidance regarding this relevant issue. ${ }^{31}$ Furthermore, in practice, clinicians are frequently faced with the decision of offering KD or another (new) AED. The effectiveness of a new AED is often similar to the effectiveness of KD and is (most often) established at lower treatment costs. In that case, it is very unlikely that positive cost-effectiveness results for KD will be reached. However, previous studies have shown that the efficacy of a new AED decreases rapidly after the failure of two to six other AEDs. Future research should focus on identifying patients (age, type and duration of the epilepsy etc.) who benefit the most from KD. Such information is crucial in allocating scarce resources in the most efficient way.

The final limitation relates to potential transferability problems of our results to other centers within and outside the Netherlands, as volumes and costs reported are (partly) related to the protocol of the tertiary epilepsy center Kempenhaeghe. 
Until now, two other published RCTs compared the KD with CAU. ${ }^{20,21}$ We found a higher percentage of responders $(50 \%)$ in the KD group compared to Neal et al. ${ }^{20}$ (38\%) and a slightly lower percentage than Sharma et al. ${ }^{21}(52 \%)$. We had more responders in the CAU group (18.2\%) than Neal et al. (6\%) and Sharma et al. (11.5\%). Our cost-utility results are in line with a recently published model-based economic evaluation that compared the KD to vagus nerve stimulation (VNS) and CAU. ${ }^{39}$ The model showed that neither KD nor VNS is a cost-effective options compared to CAU (€346,899 and €641,068 per QALY, respectively). Furthermore, Mackay et al. ${ }^{40}$ estimated the costs of 3 and 12 months of KD to be AUS\$3,879 and AUS\$7,275, respectively.

Despite a number of limitations, this study presents evidence of an economic evaluation executed alongside a RCT. We have shown that the KD is effective in terms of the number of responders. The study failed, however, to show improvements in $\mathrm{QOL}$ and, therefore, unfavorable cost-utility ratios resulted. 


\section{References}

1. Strzelczyk A, Reese JP, Dodel R, et al. Cost of epilepsy: a systematic review Pharmacoeconomics 2008;26:463-76.

2. Wallace H, Shorvon S, Tallis R. Age-specific incidence and prevalence rates of treated epilepsy in an unselected population of 2,052,922 and age-specific fertility rates of women with epilepsy Lancet 1998;352:1970-3.

3. Pugliatti M, Beghi E, Forsgren L, et al. Estimating the cost of epilepsy in Europe: a review with economic modeling Epilepsia 2007;48:2224-33.

4. Slobbe LCJ, Smit JM, Groen J, et al. Cost of Illness in the Netherlands 2007: Trends in healthcare expenditure 1999-2010 Bilthoven: RIVM; 2011.

5. Sillanpaa M, Schmidt D. Natural history of treated childhood-onset epilepsy: prospective, long-term population-based study Brain 2006;129:617-24.

6. Freeman J, Veggiotti $P$, Lanzi G, et al. The ketogenic diet: from molecular mechanisms to clinical effects Epilepsy Res 2006;68:145-80.

7. MatthewsFriends. 4th Global symposium for Dietary Thearpies for Epilepsy and other Neurological Disorders for Health Care Professionals Liverpool; 2014.

8. Caraballo RH, Cersosimo RO, Sakr D, et al. Ketogenic diet in patients with Dravet syndrome Epilepsia 2005;46:1539-44.

9. Coppola G, Veggiotti P, Cusmai R, et al. The ketogenic diet in children, adolescents and young adults with refractory epilepsy: an Italian multicentric experience Epilepsy Res 2002;48:221-7.

10. Freeman JM, Vining EP, Pillas DJ, et al. The efficacy of the ketogenic diet-1998: a prospective evaluation of intervention in 150 children Pediatrics 1998;102:1358-63.

11. Kang HC, Kim YJ, Kim DW, et al. Efficacy and safety of the ketogenic diet for intractable childhood epilepsy: Korean multicentric experience Epilepsia 2005;46:272-9.

12. Kankirawatana $P$, Jirapinyo $P$, Kankirawatana $S$, et al. Ketogenic diet: an alternative treatment for refractory epilepsy in children J Med Assoc Thai 2001;84:1027-32.

13. Kossoff EH, Pyzik PL, McGrogan JR, et al. Efficacy of the ketogenic diet for infantile spasms Pediatrics 2002;109:780-3.

14. Maydell BV, Wyllie E, Akhtar N, et al. Efficacy of the ketogenic diet in focal versus generalized seizures Pediatr Neurol 2001;25:208-12.

15. Vining EP. Clinical efficacy of the ketogenic diet Epilepsy Res 1999;37:181-90.

16. Keene DL. A systematic review of the use of the ketogenic diet in childhood epilepsy Pediatr Neurol 2006;35:1-5.

17. Lefevre F, Aronson N. Ketogenic diet for the treatment of refractory epilepsy in children: A systematic review of efficacy Pediatrics 2000;105:E46.

18. Sinha SR, Kossoff EH. The ketogenic diet Neurologist 2005;11:161-70.

19. Levy RG, Cooper PN, Giri P. Ketogenic diet and other dietary treatments for epilepsy The Cochrane Database of Syst Rev 2012;3:CD001903.

20. Neal EG, Chaffe H, Schwartz RH, et al. The ketogenic diet for the treatment of childhood epilepsy: a randomised controlled trial Lancet Neurol 2008;7:500-6.

21. Sharma S, Sankhyan N, Gulati S, et al. Use of the modified Atkins diet for treatment of refractory childhood epilepsy: a randomized controlled trial Epilepsia 2013;54:481-6.

22. Drummond MF, Sculpher MJ, Torrance GW, et al. Methods for the economic evaluation of health care programs Oxford: Oxford University Press; 2005.

23. Ligtenberg G, Staal PC, Goettsch WG, et al. Cost-effectiveness in health care Diemen: National Health Care Institute; 2013.

24. de Kinderen RJ, Lambrechts DA, Postulart D, et al. Research into the (cost-) effectiveness of the ketogenic diet among children and adolescents with intractable epilepsy: design of a randomized controlled trial BMC Neurol 2011;11:10.

25. EuroQol Group. EuroQol--a new facility for the measurement of health-related quality of life Health Policy 1990;16:199-208.

26. Wille N, Badia X, Bonsel G, et al. Development of the EQ-5D-Y: a child-friendly version of the EQ-5D Qual Life Res 2010;19:875-86. 
27. Lamers LM, McDonnell J, Stalmeier PF, et al. The Dutch tariff: results and arguments for an effective design for national EQ-5D valuation studies Health Econ 2006;15:1121-32.

28. Fekkes M, Theunissen NC, Brugman E, et al. Development and psychometric evaluation of the TAPQOL: a health-related quality of life instrument for 1-5-year-old children Qual Life Res 2000;9:96172.

29. Vogels T, Verrips GH, Koopman HM. TACQOL manual. Parent Form and Child Form Leiden: Leiden Center for Child Health and Pediatrics LUMC-TNO; 2000.

30. Goossens ME, Rutten-van Molken MP, Vlaeyen JW, et al. The cost diary: a method to measure direct and indirect costs in cost-effectiveness research J Clin Epidemiol 2000;53:688-95.

31. van Hakkaart - van Roijen L, Tan SS, Bouwmans CAM. Guideline for costing studies (in Dutch) Amstelveen: CVZ; 2010.

32. Council for Public Health and Health Care. Sensible and sustainable care (in Dutch) Zoetermeer: Council for Public Health and Health Care; 2006.

33. Salomon JA, Vos T, Hogan DR, et al. Common values in assessing health outcomes from disease and injury: disability weights measurement study for the Global Burden of Disease Study 2010 Lancet 2012;380:2129-43.

34. NICE. The guidelines manual. Process and methods guides London: National Institute for Health and Care Excellence; 2012.

35. Campo SF, Sharpton WR, Thompson B, et al. Measurement characteristics of the Quality of Life Index when used with adults who have severe mental retardation Am J Ment Retard 1996;100:546-50.

36. Birbeck GL, Hays RD, Cui X, et al. Seizure reduction and quality of life improvements in people with epilepsy Epilepsia 2002;43:535-8.

37. Stevens K, Ratcliffe J. Measuring and valuing health benefits for economic evaluation in adolescence: an assessment of the practicality and validity of the child health utility 9D in the Australian adolescent population Value Health 2012;15:1092-9.

38. Richardson JR, Peacock SJ, Hawthorne G, et al. Construction of the descriptive system for the Assessment of Quality of Life AQoL-6D utility instrument Health Qual Life Outcomes 2012;10:38.

39. de Kinderen RJ, Postulart D, Aldenkamp AP, et al. Cost-effectiveness of the ketogenic diet and vagus nerve stimulation for the treatment of children with intractable epilepsy Epilepsy Res 2015;110: 119-31.

40. Mackay MT, Bicknell-Royle J, Nation J, et al. The ketogenic diet in refractory childhood epilepsy J Paediatr Child Health 2005;41:353-7. 

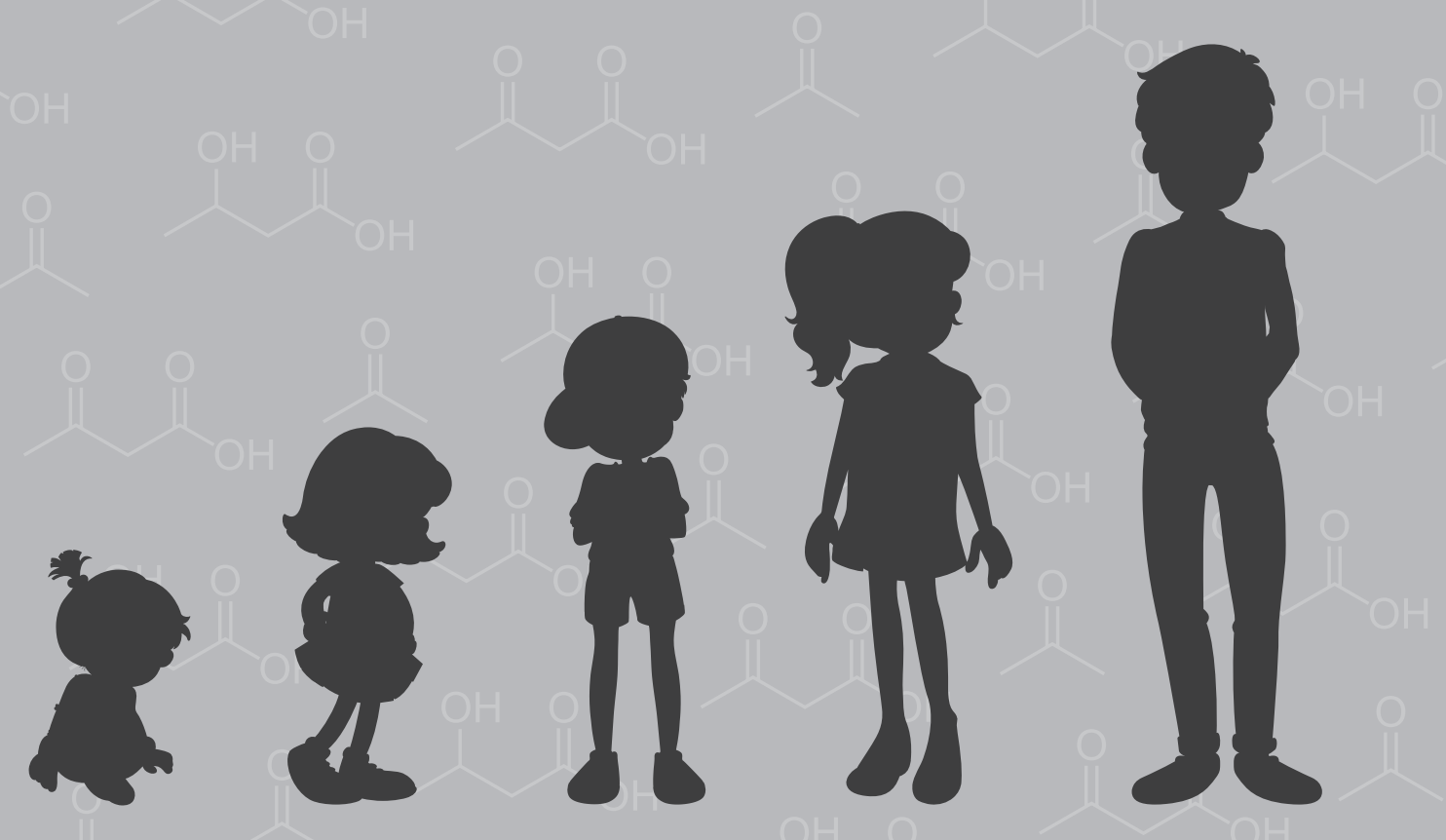


\section{Chapter 8}

\section{Blood beta-hydroxybutyrate correlates}

better with seizure reduction due to ketogenic diet than do ketones in the urine 


\section{Abstract}

\section{Purpose}

To investigate whether it is better to use blood beta-hydroxybutyrate (BHB) or urinary ketones to monitor ketogenic diet (KD).

\section{Method}

In 33 patients on KD we measured ketosis in two different ways. At the 3-monthly visits to the clinic we measured BHB in capillary blood obtained by finger-prick and the level of ketones in the urine using a urine dipstick. If the patient was able to collect urine, the urinary ketones were also measured every day at home. We compared the degree of ketosis with seizure reduction.

\section{Results}

BHB measured during the 3-monthly visits correlated with seizure reduction at 3 and 6 months ( $p=0.037$ and 0.019 , respectively). Urinary ketones measured the same time did not correlate at any visit. The averaged values of the daily measured ketones in the urine did not correlate either.

\section{Conclusions}

$\mathrm{BHB}$ correlates better with seizure reduction than do ketones in urine. It is, therefore, better to use $\mathrm{BHB}$ to monitor $\mathrm{KD}$ even if $\mathrm{BHB}$ is measured less frequently than urinary ketones. 


\section{Introduction}

It is a bizarre fact that the intake of large amounts of fat can, under certain circumstances, improve health. The ketogenic diet (KD) is an example of a healthimproving, high-fat diet applied in cases of epilepsy. Close monitoring is essential. Several monitoring methods exist, each with its own short-comings.

The KD is used in children with refractory epilepsy; the application in adults is still experimental. The diet consists mainly of fats, a sufficient quantity of protein and a small amount of carbohydrate. From former studies, it is known that the diet is effective in a third to a half of the patients. ${ }^{1-3}$

The goal of KD is to reduce seizure frequency and seizure severity. For a long time it was thought that this effect was primarily due to a rise in the level of ketones which are formed by the breakdown of the large amount of fat in the diet. Fat is consumed in the form of triglycerides which are broken down to fatty acids. In the mitochondria, these fatty acids are degraded by beta-oxidation to acetyl-CoA. Normally acetyl-CoA is used in the Krebs cycle to produce energy (ATP) which requires the presence of oxaloacetate. During KD the amount of acetyl-CoA exceeds the supply of oxaloacetate, and the resulting acetyl-CoA excess is diverted to an alternative pathway in the mitochondria which forms acetoacetic acid (AcAc), BHB and acetone, known collectively as ketone bodies. These can be used by the brain as a form of energy instead of glucose. See Figure 8.1. ${ }^{4}$

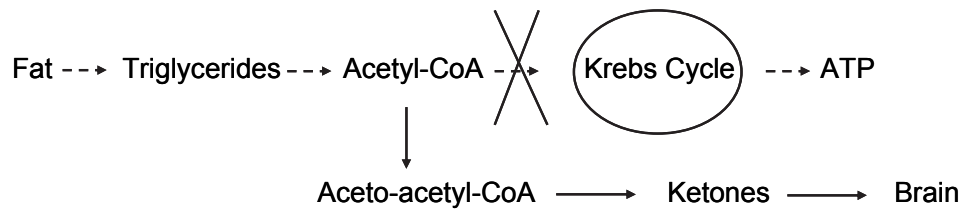

Figure 8.1 Ketone formation. Ketones are formed when acetyl-CoA is not able to enter the Krebs Cycle.

In clinical practise, ketosis is measured in two different ways: BHB is measured in blood obtained by finger-prick and the level of ketones (mainly AcAc and a small amount of acetone) can be assessed in the urine. BHB in blood seems to correlate better with the amount of ketones in the brain. ${ }^{15}$ Clinically, ketosis in the urine is usually used to monitor the diet, ${ }^{16-18}$ probably as this is less invasive.

The current view is that ketosis achieves seizure reduction. Whether this is due to a direct inhibiting action of ketones is not clear. Acetone and acetoacetate have been found to inhibit seizures in animal studies. ${ }^{5,6}$ On the other hand, beta-hydroxybutyrate (BHB) does not seem to have an anti-convulsive capability ${ }^{5}$ and none of the ketones have an influence on synaptic transmission. ${ }^{7}$ In relating BHB to seizure reduction, some researchers found that a certain amount of BHB was needed for efficacy, ${ }^{8-11}$ but others found no relationship. ${ }^{12-14}$ 
We investigated whether ketosis correlates with seizure reduction in our population. In order to establish the preferred method of monitoring KD, we measured ketosis in blood and in urine.

\section{Methods}

\section{Patients}

This study was carried out in Kempenhaeghe, a tertiary epilepsy center in the Netherlands. Since 2005, 33 patients with therapy-resistant epilepsy, aged between 1 and 40 years, have been included. Patients were excluded if there were medical contraindications (e.g. fatty acid oxidation disorders, diabetes, prolonged QT syndrome, hypercholesterolemia, liver, kidney or pancreas disease) or the expectation that compliance with the diet was not possible because of behavioral or motivational problems. Patients were excluded if they quit the diet within 6 weeks or when the seizure diaries, required to calculate efficacy, were not maintained.

\section{Study design}

Patients were admitted to the hospital for a period of 2 weeks to introduce the diet which was administered as described in the Dutch guideline for the ketogenic diet. ${ }^{19}$ This guideline does not include a fasting period prior to introduction. Usually the MCTdiet was given. If the health insurance did not refund the cost of diet products or if tube feeding was administered, the classic diet was initiated or, in adults only, the Atkins diet. In some patients changes were made in the MCT-diet, because of sideeffects and other problems encountered during its introduction. Such changes included mixing the MCT and the classic diet. All patients were put on the diet by the same dietician according to the protocol.

Seizure frequency was obtained from the diaries. A 3-month baseline was established before embarking on the diet. Reduction was calculated by comparing the number of seizures before and after starting the diet, the starting point being defined as the first change made in the normal diet. In cases with more than $50 \%$ reduction, the diet was considered effective. We did not distinguish between the various types of seizures.

\section{Ketosis}

At every 3-month visit, ketosis was measured in an early morning urine sample and in a blood sample taken between 8.00 and 12.00 . Ketosis in urine was measured using a urine dipstick, which reacts mainly with AcAc and partially with the volatile acetone. It does not cross-react with BHB. The dipstick measures ketosis semi-quantitatively, giving values of 0 to $4+$ which are related to an amount of 0 to $>16 \mathrm{mmol} / \mathrm{l}$. BHB was measured at every visit in capillary blood obtained by a finger-prick using a MediSense 
Precision Xtra. The sample is applied to the electrochemical test strip producing an electronic current, which can be measured. It has a range of $0-8 \mathrm{mmol} / \mathrm{I}$. We tried to achieve a ketosis of $3+$ to $4+(8-16 \mathrm{mmol} / \mathrm{l})$ in urine and a BHB concentration of 2-4 $\mathrm{mmol} / \mathrm{l}$ in blood.

At home, ketosis was measured on a daily basis by urine dipstick. Values were recorded and brought along to the outpatient visits every 3 months.

\section{Statistics}

Applying the SPSS programme, we related blood BHB and urinary ketones to seizure reduction using the Spearman rank correlation test. We also correlated the semiquantitative average of ketones in the urine to the seizure reduction when more than 10 samples in 3 months were available. Based on a pilot study of 6 measurements, we estimated a correlation between ketone body assessment in the urine and BHB in blood of 0.7 . To exclude values of Pearson's correlation coefficient of $<0.4$ with a power of $80 \%$ and a significance level of 0.05 one-sided, 33 patients were needed. Correlations with $\mathrm{p}<0.05$ were considered significant.

\section{Results}

The present study included 33 patients whose characteristics can be found in Table 8.1 .

Table 8.1 Characteristics of the study group.

\begin{tabular}{ll}
\hline $\mathrm{N}$ & 33 \\
\hline Age at start of diet (years), mean (min - max value) & $16.3(1.3-40.7)$ \\
$<18$ years, $\mathrm{n}(\%)$ & $19(58)$ \\
Male, $\mathrm{n}(\%)$ & $18(55)$ \\
Debut epilepsy (years), mean (min - max value) & $3.7(0.0-18.0)$ \\
Generalised seizures, $\mathrm{n}(\%)$ & $17(52)$ \\
Duration (years), mean (min - max value) & $12.5(1.3-39.3)$ \\
Amount of AED used before start of KD, mean (min - max value) & $7.9(4-14)$ \\
IQ & No retardation: 9 \\
& Mild retardation: 5 \\
Diet type & Moderate retardation: 8 \\
& Severe retardation: 11 \\
& MCT: 21 \\
\hline
\end{tabular}

Three months after the start of the study, all patients were still on the diet. At 6, 9 and 12 months there were respectively 22, 11 and 10 patients on the diet. At 3 and 6 months, respectively 5 and 6 patients showed a seizure reduction of more than $50 \%$. 
Of the 33 patients included, 8 achieved a seizure reduction of more than $50 \%$ (at 3 or 6 months) which means that in this study KD was effective in $24 \%$ of the patients. Once the diet became effective no relapse was seen in any of the patients.

When we correlated the ketosis measured at the visits with seizure reduction, we found a significant correlation for BHB in the capillary blood at 3 and 6 months ( $p=0.02$ and 0.01 ), see Table 8.2, Figures 8.2 and 8.3. Interestingly, the one patient in this study who reached complete seizure reduction on KD had a BHB comparable to those without seizure improvements. There was no correlation found with ketones measured in the urine during these visits $(p=0.44$ at 3 months and $p=0.37$ at 6 months). At 9 and 12 months, we did not find any significance for either test (data not shown).

Table 8.2 Seizure reduction related to BHB concentration.

\begin{tabular}{lcc}
\hline Reduction & $\mathrm{BHB}^{\mathrm{a}} 3$ months (SD) & $\mathrm{BHB}^{\mathrm{a}} 6$ months (SD) \\
\hline No efficacy & $\mathrm{n}$ & $0.85(0.99)$ \\
& $1.10(1.19)$ & 10 \\
$<50 \%$ reduction & 17 & $1.75(2.44)$ \\
& $1.05(0.65)$ & 5 \\
& 11 & $3.5(2.14)$ \\
Seizure free & $3.0(0.65)$ & 5 \\
& 5 & 0.3 \\
Spearman rank & 0 & 1 \\
\hline
\end{tabular}

At 6 months, 1 BHB measurement is missing. ${ }^{a}$ BHB in mmol/l.

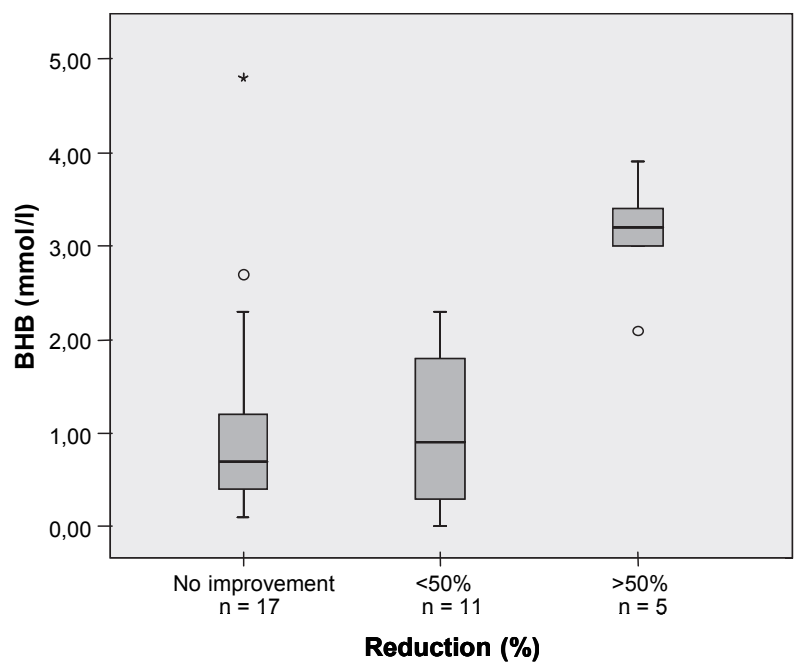

Figure 8.2 $\quad B H B$ concentration related to reduction at 3 months. 


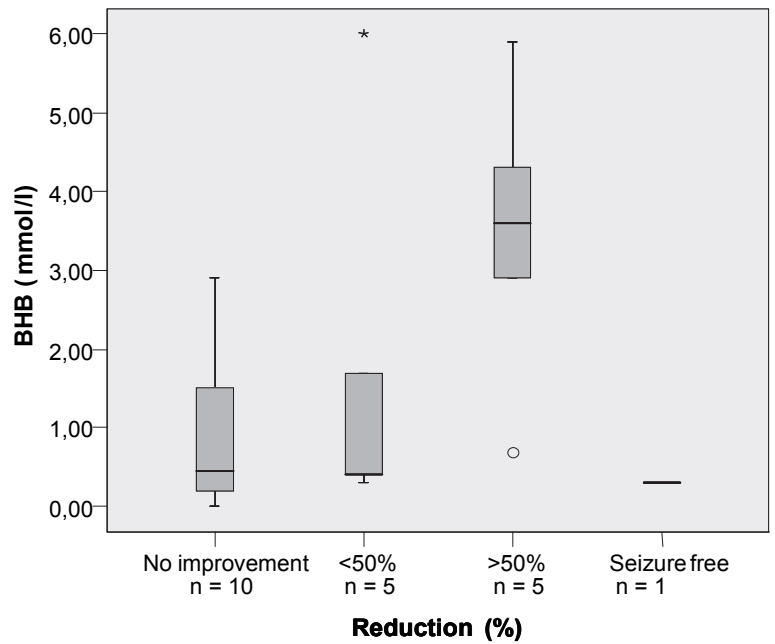

Figure 8.3 BHB concentration related to reduction at 6 months.

Normally the diet is not monitored on the basis of only one sample of urine ketosis; the ketosis measured at home is also taken into account. We, therefore, correlated the seizure reduction with the average of all the values measured by the patients at home. 25 patients at 3 months and 17 patients at 6 months had measured the ketosis frequently. But the average of several measurements of ketones in the urine did not correlate with the reduction at any time, Figures 8.4 and 8.5 .

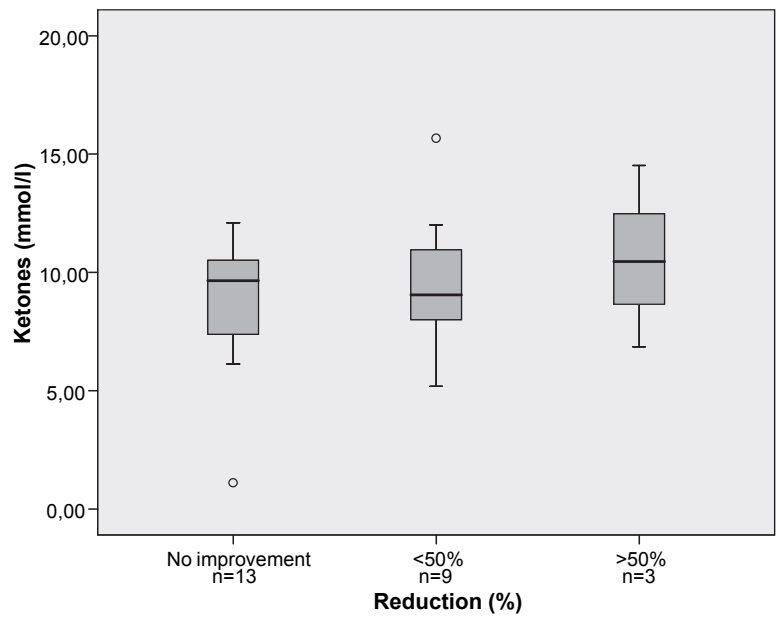

Figure 8.4 Ketones in urine (average) related to reduction at 3 months. Spearman rank: $R=0.10, p=0.32$. 


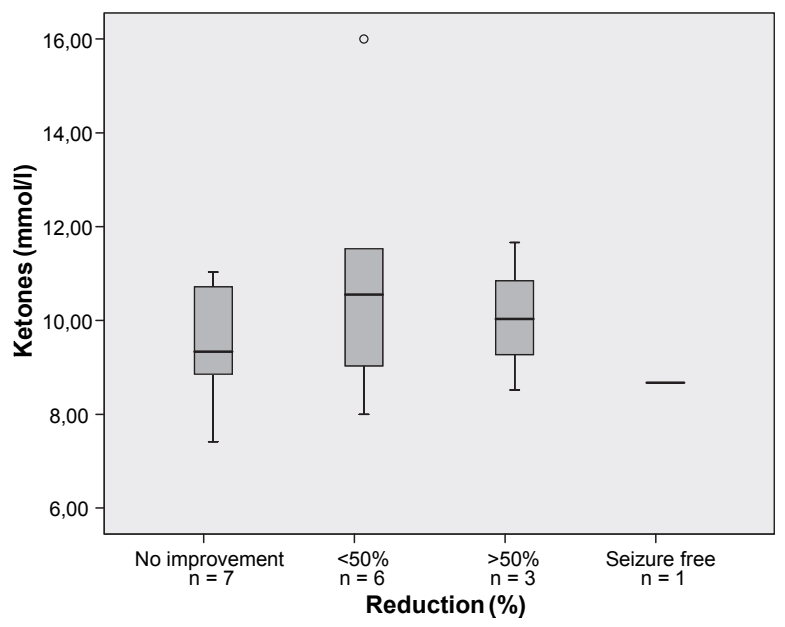

Figure 8.5 Ketones in urine (average) related to reduction at 6 months. Spearman rank: $R=0.09, p=0.36$.

\section{Discussion}

In this study we show that BHB correlates better with seizure reduction than urinary ketones. Even the average ketosis of several urine samples, measured at home, did not correlate with seizure reduction. BHB is, therefore, more useful in patients on KD. Gilbert et al. came to the same conclusion as did Likhodii et al. when studying KD in rats. ${ }^{8,11}$ Clinically, the urine dipstick is used more often to monitor KD, but our study tends to support the use of BHB rather than urine. A ketosis found in the urine only indicates compliance but it is not a value to aim for in terms of efficacy. Furthermore, fewer measurements of BHB might be required to evaluate the degree of ketosis, as only one value of $\mathrm{BHB}$ in 3 months already correlates with seizure reduction. We were not able to collect BHB and urine samples in all 33 patients. Some patients were not able to measure ketosis in the urine every day because of incontinence. There is no reason to consider the ketosis would be different in the excluded patients. Nor were we able to measure $\mathrm{BHB}$ at a fixed time due to the fact that most patients using the $K D$ have complex epileptic disorders and are often mentally retarded. All measurements were, however, carried out in the morning. Since the amount of BHB varies during the morning, this might have influenced the level, but as this is the case in both non-responders and in responders, bias may be less likely to have occurred.

The correlation with BHB was not found at 9 and 12 months, possibly due to the small number of patients in the study at that time, but Gilbert et al. came to the same conclusion. $^{8}$ We can only speculate that this indicates that $\mathrm{BHB}$ is not the ultimate parameter for monitoring the KD. Also, the only patient who became seizure free had a low level of BHB. But there is as yet no better parameter available. 
The efficacy in this study was $24 \%$, which is low compared to other KD studies. ${ }^{1-3}$ Bias may be present because caregivers may have been more eager to complete the seizure diary after the start of the KD as compared to the preceding period (baseline period). In addition, adults were also included in this study, and efficacy might be lower in adults compared to children.

We did not stratify for the form of KD (e.g. MCT, classical, Atkins), therefore, we do not know whether ketosis is less well correlated to seizure reduction in one diet compared to another. However, since ketosis was measured and the results were used in the same way in all KD, conclusions drawn in this paper are applicable to all KD.

We did not stratify for the different age groups either and therefore do not know whether ketosis in adults relates differently to seizure control compared to ketosis in children. This is due to the limited number of patients included in this study and the wide age range.

Clinicians do, however, try to reach the same level of ketosis in adults as they do in children and therefore this difference is probably not that important for the aim of the study.

In the present study we showed that infrequent BHB assessment can be used instead of daily ketone assessments in urine to monitor KD. Under certain circumstances, it shows even better correlation with seizure reduction. 


\section{References}

1. Freeman JM, Vining EP, Pillas DJ, Pyzik PL, Casey JC, Kelly LM. The efficacy of the ketogenic diet-1998: a prospective evaluation of intervention in 150 children. Pediatrics 1998;102:1358-63.

2. Neal EG, Chaffe H, Schwartz RH, Lawson MS, Edwards N, Fitzsimmons G, et al. The ketogenic diet for the treatment of childhood epilepsy: a randomised controlled trial. Lancet Neurol 2008;7:500-6.

3. Vining EP, Freeman JM, Ballaban Gil K, Camfield CS, Camfield PR, Holmes GL, et al. A multicenter study of the efficacy of the ketogenic diet. Arch Neurol 1998;55:1433-7.

4. Kim do Y, Rho JM. The ketogenic diet and epilepsy. Curr Opin Clin Nutr Metab Care 2008;11:113-20.

5. Rho JM, Anderson GD, Donevan SD, White HS. Acetoacetate, acetone, and dibenzylamine (a contaminant in I-(+)-beta-hydroxybutyrate) exhibit direct anticonvulsant actions in vivo. Epilepsia 2002;43:358-61.

6. Likhodii SS, Serbanescu I, Cortez MA, Murphy P, Snead OC3rd. Anticonvulsant properties of acetone, a brain ketone elevated by the ketogenic diet. Ann Neurol 2003;54:219-26.

7. Thio LL, Wong M, Yamada KA. Ketone bodies do not directly alter excitatory or inhibitory hippocampal synaptic transmission. Neurology 2000;54:325-31.

8. Gilbert DL, Pyzik PL, Freeman JM. The ketogenic diet: seizure control correlates better with serum beta-hydroxybutyrate than with urine ketones. J Child Neurol 2000;15:787-90.

9. Huttenlocher PR. Ketonemia and seizures: metabolic and anticonvulsant effects of two ketogenic diets in childhood epilepsy. Pediatr Res 1976;10:536-40.

10. Bough KJ, Valiyil R, Han FT, Eagles DA. Seizure resistance is dependent upon age and calorie restriction in rats fed a ketogenic diet. Epilepsy Res 1999;35:21-8.

11. Likhodii SS, Musa K, Mendonca A, Dell C, Burnham WM, Cunnane SC. Dietary fat, ketosis, and seizure resistance in rats on the ketogenic diet. Epilepsia 2000;41:1400-10.

12. Fraser DD, Whiting S, Andrew RD, Macdonald EA, Musa Veloso K, Cunnane SC. Elevated polyunsaturated fatty acids in blood serum obtained from children on the ketogenic diet. Neurology 2003;60:1026-9.

13. Todorova MT, Tandon P, Madore RA, Stafstrom CE, Seyfried TN. The ketogenic diet inhibits epileptogenesis in EL mice: a genetic model for idiopathic epilepsy. Epilepsia 2000;41:933-40.

14. Neal EG, Chaffe H, Schwartz RH, Lawson MS, Edwards N, Fitzsimmons G, et al. A randomized trial of classical and medium-chain triglyceride ketogenic diets in the treatment of childhood epilepsy. Epilepsia 2008;19:19.

15. Morris AA. Cerebral ketone body metabolism. J Inherit Metab Dis 2005;28:109-21.

16. Hartman AL, Vining EP. Clinical aspects of the ketogenic diet. Epilepsia 2007;48:31-42.

17. Kossoff EH, Zupec Kania BA,Amark PE, BallabanGil KR, Bergqvist AG, Blackford R, et al., Optimal clinical management of children receiving the ketogenic diet: Recommendations of the International Ketogenic Diet Study Group. Epilepsia 2009;50:304-17.

18. Musa-Veloso K, Cunnane SC. Measuring and interpreting ketosis and fatty acid profiles in patients on a high-fat ketogenic diet. In: Stafstrom CE, Rho JM, editors. Epilepsy and the ketogenic diet. Totowa: Humana Press; 2004:129-41.

19. Hurk van den TAM, Louw van der EJTM. Dieetbehandelingsrichtlijn ketogeen dieet voor kinderen met refractaire epilepsie. Utrecht: UMC Utrecht; 2007. 


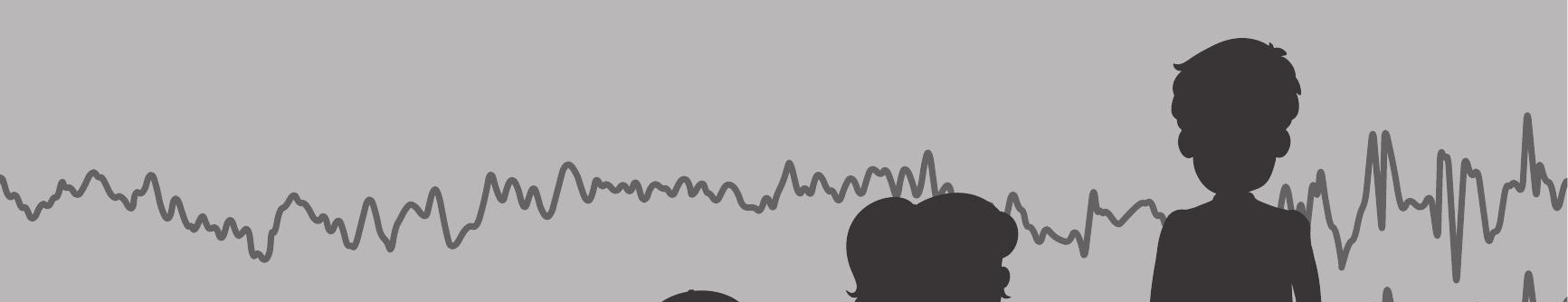

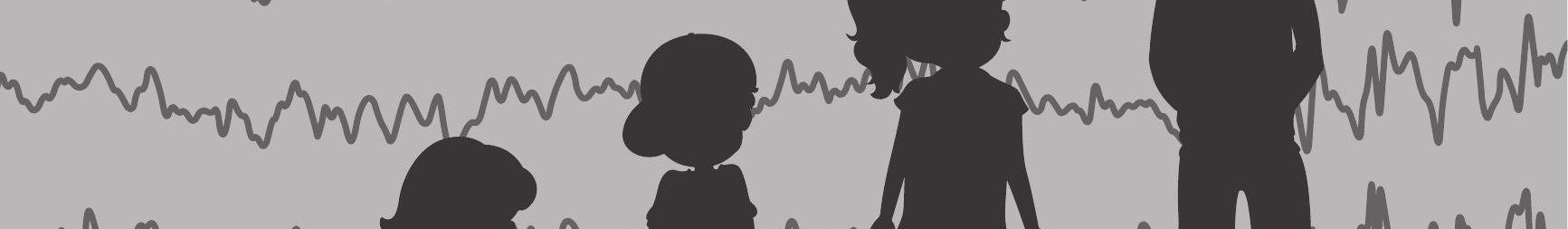

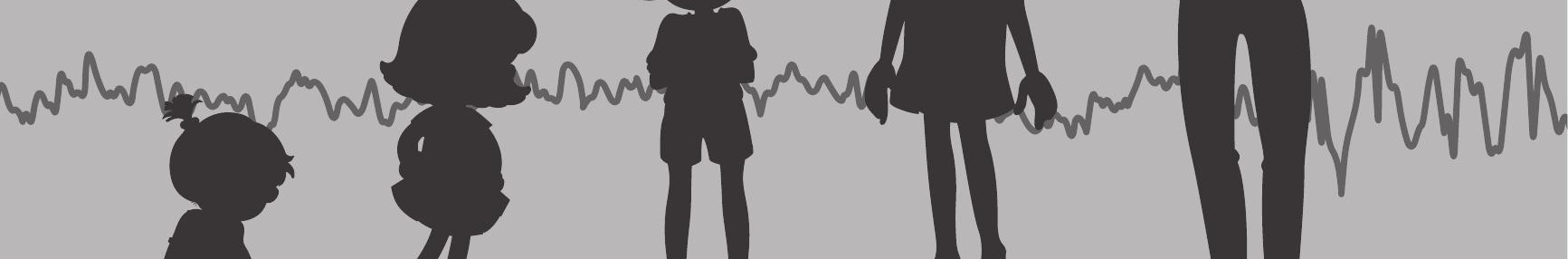




\section{Chapter 9}

Can an early 24-hour EEG predict the response to the ketogenic diet? A prospective study in $\mathbf{3 4}$ children and adults with refractory epilepsy treated with the ketogenic diet

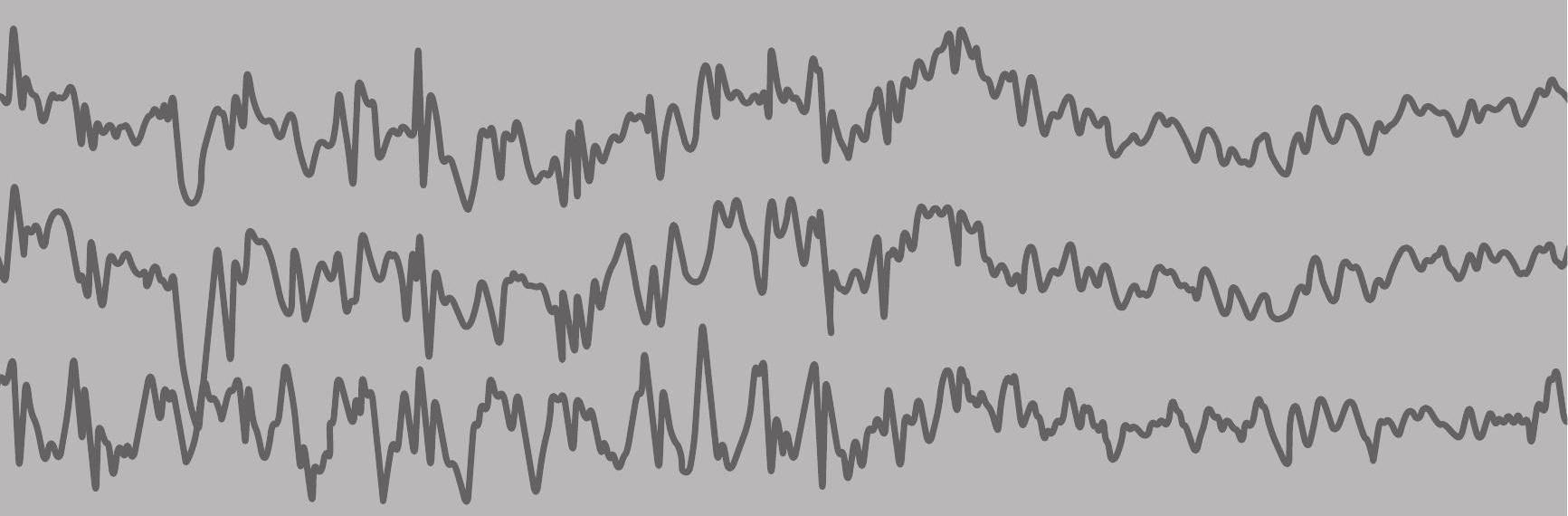

S.C.M. Ebus, D.A.J.E. Lambrechts, I.J.T. Herraets, H.J.M. Majoie, A.J.A. de Louw, P.A.J.M. Boon, A.P. Aldenkamp, J.B.A.M. Arends 


\section{Abstract}

\section{Purpose}

We examined whether early EEG changes in a 24-h EEG at 6 weeks of treatment were related to the later clinical response to the ketogenic diet (KD) in a 6-month period of treatment.

\section{Methods}

We examined 34 patients with heterogeneous epilepsy syndromes ( 21 children, 13 adults) and found 9 clinical responders ( $\geq 50 \%$ seizure reduction); this is a responder rate of $26 \%$. We visually counted the interictal epileptic discharge index (IED index) in \% during $2 \mathrm{~h}$ of wakefulness and in the first hour of sleep (method 1), and also globally reviewed EEG changes (method 2), while blinded to the effect of the KD.

\section{Results}

At group level we saw a correlation between nocturnal reduction of IED index at 6 weeks and seizure reduction in the follow-up period. A proportional reduction in IED index of $30 \%$ from baseline in the sleep EEG, was associated with being a responder to the diet (Pearson Chi-square $\mathrm{p}=0.04$ ). EEG scoring method 2 observed a significantly larger proportion of patients with EEG-improvement in sleep in KD responders than in non-responders $(p=0.03)$. At individual level, however, EEG changes did not correlate very strongly to the response to the diet, as IED reduction in sleep was also seen in $15 \%$ (method 1 ) to $26 \%$ (method 2 ) of the non-responders.

\section{Conclusion}

Nocturnal reduction of IEDs is related to the response to the KD, however in daily clinical practice, an early EEG to predict seizure reduction should not be advised for individual patients. 


\section{Introduction}

The KD is a high-fat, low-protein, low-carbohydrate diet used to treat patients with refractory epilepsy. The proportion of patients with $\geq 50 \%$ seizure reduction varies from $38-62 \%$ at 3 months after initiation of the KD to $27-58 \%$ at 6 months. ${ }^{1-6} A$ randomized, controlled trial showed that 28 patients of the KD group had greater than $50 \%$ seizure reduction (38\%), compared to four controls (6\%) and five of the KD group had greater than $90 \%$ seizure reduction (7\%), compared to none of the controls. ${ }^{7}$ There were no statistically significant differences in outcome between patients treated with the MCT or classical KD. ${ }^{7-9}$ The results of 30 studies with the MAD, of which one RCT demonstrated similar efficacy to the KD. ${ }^{7-9}$ Although it is traditionally children who are treated with the KD, there is also evidence of its efficacy in adults. ${ }^{6,10}$ The exact mechanisms underlying the effectiveness of the KD have not been elucidated. Because of the dietary changes, the KD is often experienced as a burden. If the response to the KD could be predicted in individual patients before or soon after its initiation, this could be helpful in selecting those patients who would potentially benefit from the KD and who should be encouraged to continue the diet.

The frequency of interictal epileptic discharges (IEDs) is a potential predictor for response to treatments. The occurrence of IEDs is correlated to seizure recurrence. $^{11,12}$ IED frequency is correlated with seizure-frequency, especially in patients with severe epilepsies. ${ }^{13}$ In a recent study Kessler et al. ${ }^{14}$ found that a response to the diet was 6 times more likely in children with a proportional IED index decline in wakefulness of $\geq 10 \%$ from baseline at one month of KD treatment.

In our center, adults as well as children are treated with the KD. We examined whether early EEG changes during wakefulness or sleep 6 weeks after treatment initiation could be used to predict the response to the diet in subsequent months. We hypothesized that if a reduction of IEDs is seen soon after the initiation of the KD, this is related to a reduction in seizure frequency in subsequent months. Secondly, we examined whether baseline characteristics could predict the response to the KD.

\section{Methods}

\section{Study design}

We compared the IED frequency in a 24-hour ambulatory EEG after 6 weeks of treatment with the KD, to the IED frequency in a baseline 24-hour EEG one week before treatment (Figure 9.1). A period of 4 weeks treatment is necessary to obtain stable ketosis. We registered at 6 weeks in order to be early in the treatment phase together with a high chance of all patients being in a steady ketosis at that moment. A change in IED frequency was compared with the clinical outcome as defined by the mean seizure frequency during a follow-up period ranging from at least 2 to a 
maximum of 6 months compared to 2 months baseline. Seizure counts in the first month of KD introduction were not used to define clinical outcome, because this period was necessary to obtain ketosis.

Anti-epileptic drugs (AED) were continued unchanged during baseline and the first six months of treatment with the KD. The only allowed exception was an urgent medical reason.

The study was approved by the Medical Ethics Committee according to Dutch Governmental Guidelines.

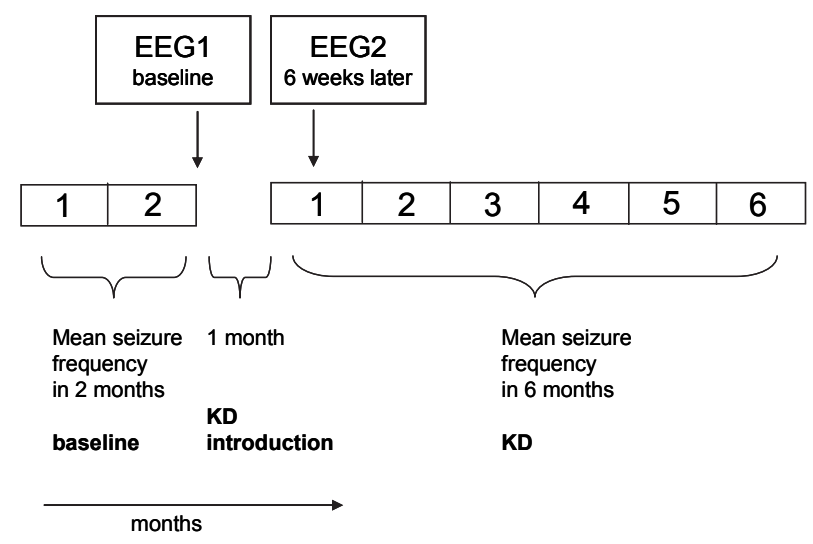

Figure 9.1 Study design.

\section{Ketogenic diet}

The KD was introduced during a 2-week hospitalization according to the Dutch guideline for KD. ${ }^{15}$ This guideline does not include a fasting period. Usually the MCT (medium chain triglyceride)-diet was applied but if the insurance company did not refund the diet products, or at the subject's request, the classical diet or modified Atkins diet (MAD) was used. Changes could be made to the MCT-diet because of sideeffects and other problems during the introduction phase. When tube feeding was given, a liquid form of the classical KD was used. The start of the diet was defined as the first change made to the daily nutrition. During the KD, ketosis was frequently measured. During the second 24-hour EEG, the ketosis was measured in urine and blood or only in blood in cases of incontinence.

\section{Clinical outcome}

Patients and/or caregivers were asked to register seizures in a diary starting two months prior to the introduction of the KD. 
The mean seizure frequency during the follow-up period after the 4 weeks of KD introduction was calculated and compared to the baseline mean seizure frequency. When the mean seizure frequency was reduced by $\geq 50 \%$, the patient was defined as being a clinical responder. If seizure diaries were incomplete, the treating physician was asked to define the patient as a responder or non-responder, using patient's and/or caregiver's information.

\section{EEG analysis}

EEGs were recorded with the localizations Fp2, F4, C4, P4, O2, F8, T4, T6, Fp1, F3, C3, $\mathrm{P} 3, \mathrm{O} 1, \mathrm{~F} 7, \mathrm{T3}, \mathrm{T} 5, \mathrm{Fp0}, \mathrm{F0}, \mathrm{CO}$ and P0. For the comparison of the IED frequency before and after KD, two EEG scoring methods were used.

\section{EEG scoring method 1}

The number of IEDs was visually counted during 2 hours of wakefulness ( 1 hour during the day and the first hour after awakening). If possible, the selection was taken from the same hours of the day for EEG 1 and EEG 2, to minimize the effects of circadian rhythm or drug level fluctuations on the IED index. The mean number of IEDs in these two hours was used to calculate a \% of time of IEDs: (number of seconds containing IEDs / total number of seconds) $* 100$ (the IED index). The IED indices from 2 EEGs (baseline and EEG at 6 weeks) were combined to calculate the proportional reduction in \% from the baseline index: (baseline index minus index at 6 weeks / baseline index) * 100. The IED index was also calculated for the first hour of sleep. The beginning of sleep was defined as the disappearance of the occipital rhythm during the previous 5 minutes. When patients did not have an occipital rhythm, the beginning of sleep was defined as the disappearance of eye-blinks during the previous 5 minutes. No IEDs were counted in the period of 30 minutes after any major seizure (tonic-clonic seizure, complex-partial seizure, or tonic seizure) to avoid possible post-ictal changes. Two EEGs from the same patient were scored directly after each other. The reviewer was blinded for the clinical outcomes.

\section{EEG scoring method 2}

A second EEG reviewer randomly scanned several parts throughout the EEG in the awake state and during the first hour of sleep. He concluded whether the EEG had the same, a lower, or a higher number of IEDs compared to the other EEG of the same patient. The reviewer was blinded for the clinical outcome.

We assumed that observed changes would be more reliable when the results of EEG scoring method 1 and EEG scoring method 2 would be congruent. Furthermore, EEG scoring method 2 resembles the daily practice of patient care. 


\section{Background analysis}

Because of the ambulatory registration, it was not possible to obtain artifact-free EEG fragments in the awake state with eyes closed for a spectral analysis. We only applied 3 global categories by visual inspection to describe the baseline EEG. These were: normal background, moderate background abnormalities (increased amount of diffuse slow activity with a preserved occipital rhythm with normal or decreased frequency), and severe background abnormalities (diffuse slowing of the EEG and absence of occipital rhythms).

\section{Patient selection}

We analyzed 34 patients, aged 1-45 years, included in the prospective study between January 2005 and September 2010.

Initially, 45 patients with refractory epilepsy who were candidates for the KD were included. In two patients an EEG could not be performed because of a safety helmet in one and behavioral problems in the other patient. One patient died of epilepsyrelated complications before the second EEG. In 7 patients, the second EEG was not registered because the diet was discontinued within 6 weeks after the baseline EEG, as a result of side-effects and/or the burden of the diet. In three patients, the second EEG was recorded; however the diet was discontinued before sufficient clinical followup had been established as was required in our study design, i.e. within two months after the 4-week introduction of the diet. Of these three, one patient had unacceptable gastro-intestinal side-effects. In the other two patients, severe seizure aggravation, not expected from earlier seizure observations (occurrence of at least twice as many seizures in both patients and additional frequent non-convulsive epileptic status in one of them), led to early discontinuation. We decided to analyze these two patients as non-responders.

\section{Statistics}

We used Mann-Whitney $U$ test (MWU) for calculating differences between groups, Spearman's correlations for correlations between 2 variables, and Pearson Chi-square test (2-sided) to test differences in proportions. Results were considered significant if alpha was $\leq 0.05$.

\section{Results}

\section{Baseline data}

The data of the 34 patients are shown in Tables 9.1a-9.1c. 
Table 9.1a Demography.

\begin{tabular}{lc}
\hline Patients, N & 34 \\
\hline Male/female, N (\%) & $17 / 17(50 \% / 50 \%)$ \\
Mean age at KD initiation in years;months (min-max value) & $16 ; 0(1 ; 1-45 ; 5)$ \\
Number of children (age $<18$ years) at KD initiation, N (\%) & $21(62 \%)$ \\
Mean age at seizure onset in years; months (min-max value) & $5 ; 0(0 ; 0-31 ; 6)$ \\
Mean duration of epilepsy in years; months (min-max value) & $11 ; 0(1 ; 3-44 ; 11)$ \\
Number of AEDs ${ }^{1}$ at KD initiation & $2.4(0-5)$ \\
Number of used AED before KD initiation & $7.3(4.0-14.0)$ \\
Number of patients with VNS treatment in the past & $3(9 \%)$ \\
Seizure frequency at KD initiation & \\
Daily seizures & $19(56 \%)$ \\
$\geq 1$ seizure a week & $10(29 \%)$ \\
$\geq 1$ seizure a month & $5(15 \%)$ \\
Diet type & \\
Classic, N (\%) & $8(24 \%)$ \\
MCT, N (\%) & $19(56 \%)$ \\
Atkins, N (\%) & $2(6 \%)$ \\
Mixed, N (\%) & $5(14 \%)$ \\
\hline
\end{tabular}

${ }^{1} \mathrm{AED}=$ anti-epileptic drug.

Table 9.1b Syndromal diagnosis.

\begin{tabular}{|c|c|c|c|c|}
\hline Syndromal diagnosis & Subtype / etiology & $\begin{array}{l}\text { Number of } \\
\text { patients }\end{array}$ & $\begin{array}{l}\text { Number of } \\
\text { patients } \\
\text { (total of } \\
\text { subgroup) }\end{array}$ & Percentage \\
\hline $\begin{array}{l}\text { Cryptogenic localization- } \\
\text { related epilepsy }\end{array}$ & & 17 & 17 & $50 \%$ \\
\hline \multirow{10}{*}{$\begin{array}{l}\text { Symptomatic localization- } \\
\text { related epilepsy }\end{array}$} & Perinatal asphyxia & 3 & 14 & $41 \%$ \\
\hline & Bandheterotopia & 1 & & \\
\hline & Bacterial meningo-encephalitis & 3 & & \\
\hline & Trauma & 1 & & \\
\hline & Partial trisomy 14 and 8 & 1 & & \\
\hline & Sturge Weber syndrome & 1 & & \\
\hline & CDKL-5 mutation & 1 & & \\
\hline & Perinatal intracerebral haemorrhage & 1 & & \\
\hline & Down syndrome & 1 & & \\
\hline & $1 p$-syndrome & 1 & & \\
\hline $\begin{array}{l}\text { Symptomatic generalized } \\
\text { epilepsy }\end{array}$ & $\begin{array}{l}\text { Myoclonic astatic epilepsy (Doose } \\
\text { syndrome) }\end{array}$ & 2 & 2 & $6 \%$ \\
\hline $\begin{array}{l}\text { Undetermined epilepsies } \\
\text { with both generalized and } \\
\text { focal seizures }\end{array}$ & $\begin{array}{l}\text { Severe myoclonic epilepsy (Dravet } \\
\text { syndrome) }\end{array}$ & 1 & 1 & $3 \%$ \\
\hline Total & & 34 & 34 & $100 \%$ \\
\hline
\end{tabular}


Table 9.1c EEG baseline characteristics.

\begin{tabular}{|c|c|c|c|}
\hline & Categories & $\mathrm{Nr}$ of patients & Mean IED index ${ }^{1}$ \\
\hline \multirow[t]{4}{*}{ IEDs in wakefulness } & No IEDs in wakefulness & $4(12 \%)$ & $0 \%$ \\
\hline & IEDs in wakefulness & $30(88 \%)$ & $6.2 \%$ (SD $18.6 \%)$ \\
\hline & Sporadic IEDs ( $\leq 1 \%)$ & 17 (50\%) & $0.15 \%$ (SD $0.18 \%$ ) \\
\hline & Non-sporadic IEDs (>1\%) & $13(38 \%)$ & $16.1 \%$ (SD $28.0 \%$ ) \\
\hline \multirow[t]{5}{*}{ IEDs in sleep } & No IEDs in sleep & $7(21 \%)$ & $0 \%$ \\
\hline & IEDs in sleep & $26(76 \%)$ & $15.1 \%$ (SD $25.8 \%$ ) \\
\hline & Sporadic IEDs ( $\leq 1 \%)$ & $6(17 \%)$ & $0.50 \%$ (SD $0.28 \%$ ) \\
\hline & Non-sporadic IEDs (>1\%) & 20 (59\%) & $24.7 \%$ (SD $21.5 \%$ ) \\
\hline & Missing data & $1(3 \%)$ & \\
\hline \multirow[t]{3}{*}{ Background abnormality } & No & $8(24 \%)$ & \\
\hline & Moderate & $18(53 \%)$ & \\
\hline & Severe & $8(23 \%)$ & \\
\hline
\end{tabular}

${ }^{1}$ Obtained by EEG scoring method 1.

$62 \%$ of the patients were children. $75 \%$ had at least several seizures a week, and about half of the patients had one or more daily seizures.

24 Patients were on the KD for at least 6 months, 8 patients discontinued the diet between 2 and 6 months following the 4 weeks of KD introduction, and 2 patients discontinued the diet between the end of the 4 weeks of KD introduction and 2 months follow-up. Seizure diaries were complete for 25 patients at baseline and 23 patients at follow-up. In 11 patients the clinical response to the diet had to be defined by the treating physician because of incomplete diaries.

$88 \%$ of the patients had IEDs in the baseline awake EEG and $76 \%$ of the patients had IEDs in the baseline sleep EEG. 21 Patients had no or only sporadic IEDs ( $\leq 1 \%)$ at baseline in the awake state.

There was a trend towards correlation between baseline seizure frequency and IED index (wakefulness Spearman's $R=0.37 p=0.07$; sleep Spearman's $R=0.36 p=0.09$ ). 76\% of the EEGs showed a moderate to severe background abnormality. The severity of background abnormality was correlated to baseline seizure frequency (Spearman's $R=0.45 p=0.02$ ), and to baseline IED index in wakefulness (Spearman's $R=0.36 p=0.04$ ) and in sleep (Spearman's $R=0.35 \mathrm{p}=0.05$ ).

\section{Results after KD introduction}

\section{Seizure outcome}

$9 / 34$ patients ( $26 \%$ ) were clinical responders ( $\geq 50 \%$ seizure reduction), of whom 7 were children. In 7 of the clinical responders the response was diary-defined, in 2 physician-defined because of an incomplete diary. They all had had a follow-up period of 6 months. The 7 responders with diaries showed a clear seizure reduction from the second month of treatment which lasted until 6 months of follow-up, resulting in a 
mean seizure reduction of $\geq 50 \%$ during 6 months, compared to the 2 months baseline period.

The mean baseline monthly seizure frequency in these 7 responders with diaries was 88.6 (SD 78.3) and in 16 non-responders with diaries 60.4 (SD 84.0). Mean seizure frequency in the follow-up period in 7 responders was 12.4 (SD 23.4) and in 16 nonresponders with diaries 68.9 (SD 85.2). Mean age of clinical responders was 10.9 (SD 12.1) and of non-responders 18.3 (SD 11.2). Mean duration of epilepsy in clinical responders was 6.6 years (SD 5.4) and in non-responders 13.0 years (SD 11.0).

There was no correlation between the clinical response to the KD and baseline seizure frequency. There was a correlation between the clinical response to the KD and younger age $(R=0.34 p=0.05)$ and a trend towards correlation between the clinical response and shorter duration of epilepsy $(R=0.31 p=0.08)$.

\section{EEG analysis at group level}

A summary of the main findings is presented in Table 9.2.

Table 9.2 Summary of findings.

\begin{tabular}{|c|c|c|}
\hline & Negative findings & Positive findings \\
\hline \multirow[t]{2}{*}{ Group level } & $\begin{array}{l}\text { No correlation between baseline IED }{ }^{1} \\
\text { index (wakefulness/sleep) or EEG } \\
\text { background disturbance and effect of } \\
\text { KD }\end{array}$ & $\begin{array}{l}\text { Seizure reduction in follow-up period } \\
\text { correlated with IED reduction in sleep } \\
\text { EEG at } 6 \text { weeks KD }(R=0.56 p=0.04)\end{array}$ \\
\hline & $\begin{array}{l}\text { No significant change in mean awake or } \\
\text { sleep IED index after } 6 \text { weeks KD }\end{array}$ & \\
\hline Individual level & See results section & $\begin{array}{l}\text { EEG scoring }^{3} \text { method } 1 \\
\text { Proportional reduction }^{2} \text { of } \geq 30 \% \text { of IED } \\
\text { index in sleep correlated to effect of KD } \\
(p=0.04) \\
\text { EEG scoring }{ }^{4} \text { method } 2 \\
\text { IED reduction in sleep correlated to effect } \\
\text { of } K D(p=0.04)\end{array}$ \\
\hline
\end{tabular}

${ }^{1} \mathrm{IED}=$ interictal epileptic discharges; ${ }^{2}$ proportional reduction $=$ (baseline IED index minus index at 6 weeks/baseline index) ${ }^{*} 100 ;{ }^{3}$ IEDs counted in first hour of sleep; ${ }^{4}$ Global review (are IEDs reduced or not ?).

31 patients were in a ketotic state on the day of the EEG. In 2 patients ketosis was measured within the two days of the EEG and in 1 patient one week after the EEG. Since these values confirmed a ketotic state we assumed that this was also the case on the day of the EEG.

AED daily doses were unchanged between the two EEG's except for one patient with a minimal dose reduction of one AED. 
We did not find correlations between the severity of EEG background slowing at baseline or the IED index at baseline and response to the KD. There was no correlation between the baseline IED index and the \% of seizure reduction.

We found a positive correlation between the \% of IED reduction in sleep at 6 weeks and the $\%$ of seizure reduction in the follow-up period (Spearman's $R=0.56 p=0.04$ ) (see Figure 9.2) but not for IED reduction in wakefulness (Spearman's $R=0.2 p=0.4$ ).

We did not find a change in mean IED index after 6 weeks KD, in either wakefulness or in sleep ( $n=34$ patients MWU wakefulness $p=0.67, n=33$ patients MWU sleep $p=0.78$ ). Mean awake IED index at follow-up was $6.3 \%$ (SD $17.0 \%$ ) vs. baseline index $6.3 \%$ (SD $18.6 \%$ ) and mean sleep IED index at follow-up was $14.8 \%$ (SD 24.3\%) vs. baseline index 15.1\% (SD 25.8\%).

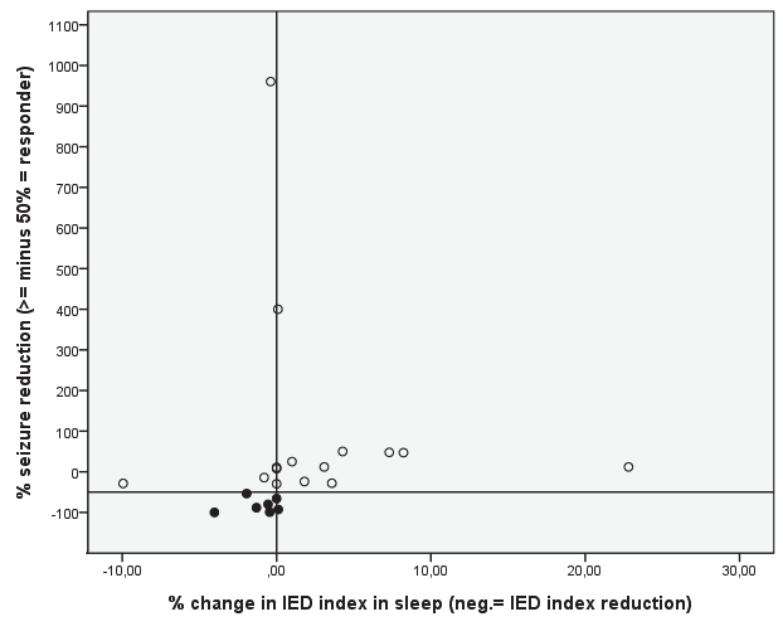

Figure 9.2 Seizure reduction versus reduction in IED index, sleep.

All (bold) dots on or below the horizontal line are patients with $\geq 50 \%$ seizure reduction (clinical responders). All dots left of the vertical line are patients with IED reduction (according to EEG scoring method 1 ). Spearman's $R=0.56 \mathrm{p}=0.04$.

\section{EEG analysis at individual level}

In 4/34 patients, no IEDs were seen during the 24-hour EEG at baseline or at follow-up according to EEG scoring method 1 (2 responders, 2 non-responders). In the remaining 30 patients with IEDs in wakefulness ( 7 clinical responders, 23 clinical nonresponders), and in the 26 patients with IEDs during sleep (7 clinical responders, 19 non-responders), we analyzed which proportions of responders vs. non-responders had changes in IED index at follow-up. 
Individual analysis of the EEG in wakefulness

Disappearance of IEDs in wakefulness according to EEG scoring method 1 was observed in 6 patients ( 2 responders, 4 non-responders). They had very low baseline IED indices between 0.01 and $1.22 \%$. In these patients with low baseline index, there was a discrepancy in confirming lack of IEDs at baseline or follow-up between EEG scoring methods 1 and 2. EEG scoring method 2 confirmed the lack of IEDs at followup in 2 of the 6 patients identified by scoring method 1. In these 2 patients, however, method 2 had not observed IEDs in the baseline EEG either.

Twelve out of 30 patients (2/7 responders, 10/23 non-responders) had a reduction (not disappearance) of IEDs according to method 1 . This did not distinguish responders from non-responders $(p=0.48)$. EEG scoring method 2 confirmed the reduction of IEDs in 6 of these patients with proportional reductions in excess of $20 \%$. EEG scoring method 2 did not distinguish between responders and non-responders (2/7 responders, $4 / 23$ non-responders $(p=0.52)$.

Disappearance of IEDs or IED-reduction according to method 1 was observed in 4/7 responders with IEDs at baseline and in $14 / 23$ non-responders (not significant $p=0.86$ ). In order to compare our findings with the findings in the study by Kessler et al., ${ }^{14}$ we examined which patients had a proportional reduction of at least $10 \%$ in wakefulness according to method 1 . This did not distinguish the responders (4/7) from nonresponders $(13 / 23)(p=0.98)$. Nor could we distinguish between responders and nonresponders when higher proportional reductions were required.

Individual analysis of the EEG in sleep

Among the 26 patients with IEDs in sleep at baseline, there were no patients in whom IEDs disappeared. We observed a reduction of IED index according to EEG scoring method 1 in 12/26 patients (5/7 responders and $7 / 19$ non-responders $(p=0.12)$. The 7 non-responders had a higher mean baseline index (32.3\% SD 24.8) than the 5 responders (4.1\% SD 4.3\%), which was the attribution of 4 non-responders with high baseline index in sleep between 18.9 and $87.9 \%$. The other 3 non-responders were comparable to the responders with a mean baseline index of $3.4 \%$ (SD 3.2\%). Although these data seem to point to a better result of the KD in patients with low IED index in sleep, this was not proved (see above "EEG analysis at group level").

EEG scoring method 2 found a reduction of IEDs in 5/7 responders and in 5/19 nonresponders, which was a significant difference $(p=0.04)$.

EEG scoring method 2 confirmed the reduction of IEDs found by method 1 in 9 of the 12 patients (4 responders, 5 non-responders). In the 3 patients in whom EEG method 2 did not confirm the IED reduction, this was explained by a very low baseline sleep index $<1 \%$ in 2 patients ( 1 responder, 1 non-responder), and by a very low IED reduction of $0.81 \%$ (proportional reduction $11.9 \%$ ) in 1 patient with baseline index $6.9 \%$. 
We finally examined whether a certain proportional reduction of the IED index during sleep could identify responders. We found a significant difference between responders and non-responders when a proportional reduction of at least $30 \%$ was required, which was observed in $4 / 7$ responders vs. $3 / 19$ non-responders $(p=0.04)$. All these 7 patients also had proportional reductions in wakefulness exceeding $20 \%$. The syndromal diagnoses of the 4 clinical responders with at least $30 \%$ proportional reduction in nocturnal IED index were: cryptogenic localisation related epilepsy $n=2$, symptomatic epilepsy after cerebral trauma $n=1$, symptomatic epilepsy after asphyxia $\mathrm{n}=1$. They were 3 children and 1 adult. They all had focal interictal epileptiform activity.

\section{Patients with increase in IED index}

We examined whether an increase in IED index would be observed more frequently in non-responders than in responders. In wakefulness this was observed in $3 / 7$ responders and in 9/23 non-responders according to EEG scoring method $1(p=0.86)$. EEG scoring method 2 identified $2 / 7$ responders with increase in IEDs in wakefulness vs. $8 / 23$ non-responders $(p=0.76)$. Increase in IED index in sleep according to EEG scoring method 1 was observed in 2/7 responders and 12/23 non-responders $(p=0.27)$. EEG scoring method 2 observed 11 patients (1 responder, 10 nonresponders) with increase in IEDs ( $p=0.16)$.

\section{Discussion}

Nine of 34 patients ( 7 children and 2 adults) were clinical responders, which represents a responder rate of $26 \%$ (33\% in the children, $15 \%$ in adults). There were no baseline EEG features that characterized future clinical responders. At a group level we identified a correlation between a nocturnal reduction of IEDs at 6 weeks and a seizure reduction in the follow-up period. More relevant for clinical practice are changes in an early EEG at the individual level. At six weeks, EEG scoring method 1 (IED counts) found that a $30 \%$ proportional reduction of the IED index in sleep at 6 weeks, was seen more often in responders than in non-responders. EEG scoring method 2 (global reviewing) also found a significantly larger proportion of patients showing IED reduction in sleep within the group of clinical responders. The fact that the two different types of EEG scoring methods found a significant correlation between IED reduction in sleep and clinical response, adds to the reliability of finding this correlation. We observed that global reviewing is less sensitive for the detection of changes. It detected changes when spike-counts had resulted in $\geq 20 \%$ IED reduction from baseline. The only clinical correlation we observed was correlated with $\geq 30 \%$ IED reduction from baseline. Therefore, global reviewing (as it is performed in clinical daily practice), may be a good alternative for the time-consuming method of 
counting individual spikes. We found however that for individual patients the observation of reduction of IEDs in this study was not a very powerful predictor, as this was also observed in $16 \%$ (method 1 ) to $26 \%$ (method 2 ) of the non-responders. We conclude that this observation cannot reliably be used to advise patients whether to continue or discontinue the diet.

We included a large proportion of patients with a low baseline IED index in wakefulness. This explains why we found the highest reductions of IEDs during sleep. In patients with a low baseline index in wakefulness, there were large discrepancies in the findings when EEG methods 1 and 2 were compared. This illustrates that outcomes on changes in this group are less reliable and are dependent on which samples of the EEG were assessed. It is not likely that reviewing every page of the awake EEG of patients with a very low IED index, would have produced a better distinction between responders and non-responders. In patients with higher baseline index (>1.22\%) and with higher proportional decreases in IED index (above $20 \%$ ) the two scoring methods had congruent results on detecting changes.

A weakness in studies with the EEG as outcome parameter is that there is no information on fluctuations of the IED index outside the chosen duration of the EEG registration. We performed a long-term EEG, but we were not informed on day-to-day fluctuations when treatment would not be changed. We cannot exclude that a proportional reduction of at least $30 \%$ can be a result of spontaneous fluctuations.

In 6 other studies, ${ }^{4,14,16-19}$ IED frequency changes after KD were analyzed and compared to a baseline. Only one study analyzed the IED index during sleep (at group level) and found a significant reduction of IEDs at 3 months, as well as a positive correlation between IED frequency reduction and seizures. This last observation corresponds to our findings. All patients in the 6 previously published studies were children. Three studies examined individual EEG changes in responders vs. nonresponders during wakefulness. Remahl et al. ${ }^{17}$ described an IED reduction in 8/16 responders vs. $5 / 7$ non-responders (not significant). Dressler et al. ${ }^{18}$ found disappearance of IEDs in $9 / 25$ responders vs. $1 / 24$ non-responders $(p=0.009)$. Kessler et al. ${ }^{14}$ found an individual IED proportional index decline of $\geq 10 \%$ of the baseline index in 12/26 responders and in 2/11 non-responders (with a baseline median spike index of 7.7). The timing and analysis of the EEG in this study correspond fairly well to our method. We could not, however, reproduce the same results for the EEG in wakefulness. Our patient group had a much lower baseline median spike index of $0.2 \%$ (mean $6.2 \%$ ) compared to the patient group of Kessler. In fact most cited studies had included more patients with generalized, highly frequent IEDs in wakefulness. Especially in patients with generalized epilepsy syndromes such as Lennox-Gastaut syndrome, myoclonic astatic epilepsy and West syndrome, highly frequent IEDs in the awake state are usually observed. We only included $2 / 34(6 \%)$ of such patients, Kessler $^{14}$ 6/37 (16\%), Remahl ${ }^{17} 5 / 23$ (22\%) and Dressler ${ }^{18}$ 15/50 (30\%). Furthermore, Kessler classified a total of $18 / 37$ (49\%) of patients with a "generalized electroclinical syndrome". Dressler classified 46/50 patients with a "generalized epilepsy syndrome". 
Finally, earlier studies included only children, this study also adults. The differences in the epilepsy syndromes, EEG-types and ages probably explain the different findings in the studies.

From the combined results of all studies, it is clear that decreases in IED index can be found in a repeated EEG in individual patients on the KD with some correlation with the effect of the KD. Changes in IED index are best observed in patients with epilepsy syndromes with high baseline IED index. The studies show however that an individual level, the method of performing an early EEG to predict whether the patient will be a responder, is not conclusive. Furthermore, in our study a clear and stable seizure reduction in the clinical responders could already be observed in the second month of KD treatment. Hence, in our patient group, an early EEG at 6 weeks as a predictor of future seizure reduction, was not necessary. Kessler ${ }^{14}$ commented that "the practical utility of performing an early EEG as predictor, may be limited to those patients whose seizure reduction on the KD is not immediate". The number of patients with a delayed reduction of seizures in this study was, however, not mentioned. There are several studies confirming that early seizure reduction (from the $1^{\text {st }}$ month of treatment) is seen far more often than delayed seizure reduction (Coppola et al. ${ }^{1}$ Bergvist et al. ${ }^{20}$, Mosek et al. ${ }^{21}$ ).

In our study, we did not observe complete normalization of the EEG in patients with a baseline IED index exceeding $1.22 \%$. Some studies mention abolition of frequent IEDs after KD, for example, in patients with West syndrome and hypsarrhythmia. ${ }^{22,23}$ Nikanorova et al. ${ }^{24}$ studied 4 children with CSWS (syndrome of continuous spike waves in slow wave sleep) in whom IEDs were recorded during sleep in $\geq 85 \%$ of the time. After 12 months of treatment, the EEG had normalized in one patient, slightly improved in one patient and not improved in 2 patients. The real "EEG-responder" showed a slight increase in IQ scores, disappearance of aggression and an improvement in concentration at that time. When clinical effects are subtle and not (yet) obvious, the normalization of the EEG may encourage such patients to continue the KD. The previously mentioned studies show that effects of the KD on the EEG could be more profound in certain syndromes. In patients with GLUT-1 deficiency (the best known indication for the KD), reduction of IEDs was reported in all 4 patients who showed IEDs at baseline, of whom 3 had $>90 \%$ seizure reduction ( 1 had never experienced seizures). ${ }^{25}$ The baseline frequency of IEDs and the magnitude of the reduction were not mentioned in this study. 


\section{Conclusion}

Although we observed a correlation between seizure reduction and IED reduction during sleep at group level, we found no evidence that early EEG changes predict the efficacy of the KD in individual patients. Only in certain patients, should EEG registration before and after KD still be considered. These are patients with highly frequent interictal epileptic discharges which are a major goal of treatment, such as patients with hypsarrhythmia or ESES (electrical status epilepticus of slow wave sleep). In all other patients, the observation of seizure reduction, which will most often be noted early in the follow-up period, is the best parameter for evaluating the effect of the KD. 


\section{References}

1. Coppola G, Veggiotti P, Cusmai R, Bertoli S, Cardinali S, Dionisi-Vici C, Elia M, Lispi ML, Sarnelli C, Tagliabue A, Toraldo C, Pascotto A. The ketogenic diet in children, adolescents and young adults with refractory epilepsy: an Italian multicentric experience. Epilepsy Res 2002;48:221-7.

2. Vining EP, Freeman JM, Ballaban-Gil K, Camfield CS, Camfield PR, Holmes GL, Shinnar S, Shuman R, Trevathan E, Wheless JW. A multicenter study of the efficacy of the ketogenic diet. Arch Neurol 1998;55:1433-7.

3. Maydell BV, Wyllie E, Akhtar N, Kotagal P, Powaski K, Cook K, Weinstock A, Rothner AD. Efficacy of the ketogenic diet in focal versus generalized seizures. Pediatr Neurol 2001;25:208-12.

4. Kang HC, Kim YJ, Kim DW, Kim HD. Efficacy and safety of the ketogenic diet for intractable childhood epilepsy: Korean multicentric experience. Epilepsia 2005;46:272-9.

5. Freeman JM. Seizures, EEG events, and the ketogenic diet. Epilepsia 2009;50:329-30.

6. Sirven J, Whedon B, Caplan D, Liporace J, Glosser D, O'Dwyer J, Sperling MR. The ketogenic diet for intractable epilepsy in adults: preliminary results. Epilepsia 1999;40:1721-6.

7. Neal EG, Chaffe H, Schwartz RH, Lawson MS, Edwards N, Fitzsimmons G, Whitney A, Cross JH. The ketogenic diet for the treatment of childhood epilepsy: a randomised controlled trial. Lancet Neurol 2008;7:500-6.

8. Sharma S, Sankhyan N, Gulati S, Agarwala A. Use of the modified Atkins diet for treatment of refractory childhood epilepsy: a randomized controlled trial. Epilepsia 2013;54:481-6.

9. Kossoff EH, Cervenka MC, Henry BJ, Haney CA, Turner Z. A decade of the modified Atkins diet (20033013): Results, insights, and future directions. Epilepsy and Behavior 2013;29:437-42.

10. Payne NE, Cross JH, Sander JW, Sisodiya SM. The ketogenic and related diets in adolescents and adults--a review. Epilepsia 2011;52:1941-8.

11. Das CP, Sawhney IM, Lal V, Prabhakar S. Risk of recurrence of seizures following single unprovoked idiopathic seizure. Neurol India 2000;48:357-60.

12. Berg AT, Shinnar S. The risk of seizure recurrence following a first unprovoked seizure: a quantitative review. Neurology 1991;41:965-72.

13. Ebus SC, Majoie HJ, Arends JB, Boon PJ. Can spikes predict seizure frequency? Results of a pilot study in severe childhood epilepsies treated with vagus nerve stimulation. Seizure 2004;13:494-8.

14. Kessler SK, Gallagher PR, Shellhaas RA, Clancy RR, Bergqvist AG. Early EEG improvement after ketogenic diet initiation. Epilepsy Res 2011;94:94-101.

15. Hurk van den TAM, Louw van der EJTM. Dieetbehandelingsrichtlijn ketogeen dieet voor kinderen (018) met refractaire epilepsie. Evidence based handleiding voor een multidisciplinaire behandeling. Utrecht: UMC Utrecht: 2007.

16. Hallböök T, Kohler S, Rosen I, Lundgren J. Effects of ketogenic diet on epileptiform activity in children with therapy resistant epilepsy. Epilepsy Res 2007;77:134-40.

17. Remahl S, Dahlin MG, Amark PE. Influence of the ketogenic diet on 24-hour electroencephalogram in children with epilepsy. Pediatr Neurol 2008;38:38-43.

18. Dressler A, Stöcklin B, Reithofer E, Benninger F, Freilinger M, Hauser E, Reiter-Fink E, Seidl R, TrimmelSchwahofer $\mathrm{P}$, Feucht $\mathrm{M}$. Long-term outcome and tolerability of the ketogenic diet in drug-resistant childhood epilepsy--the Austrian experience. Seizure 2010;19:404-8.

19. Li B, Tong L, Jia G, Sun R. Effects of ketogenic diet on the clinical and electroencephalographic features of children with drug therapy-resistant epilepsy. Exp Ther Med 2013;5:611-15.

20. Bergqvist AG, Schall JI, Gallagher PR, Cnaan A, Stallings VA. Fasting versus gradual initiation of the ketogenic diet: a prospective, randomized clinical trial of efficacy. Epilepsia 2005;46:1810-9.

21. Mosek A, Natour H, Neufeld MY, Shiff Y, Vaisman N. Ketogenic diet treatment in adults with refractory epilepsy: a prospective pilot study. Seizure 2009;18:30-3.

22. Eun SH, Kang HC, Kim DW, Kim HD. Ketogenic diet for treatment of infantile spasms. Brain Dev 2006;28:566-71.

23. Hong AM, Turner Z, Hamdy RF, Kossoff EH. Infantile spasms treated with the ketogenic diet: prospective single-center experience in 104 consecutive infants. Epilepsia 2010;51:1403-7.

24. Nikanorova M, Miranda MJ, Atkins M, Sahlholdt L. Ketogenic diet in the treatment of refractory continuous spikes and waves during slow sleep. Epilepsia 2009;50:1127-31. 
25. Ito $\mathrm{Y}$, Oguni $\mathrm{H}$, Ito $\mathrm{S}$, Oguni M, Osawa M. A modified Atkins diet is promising as a treatment for glucose transporter type 1 deficiency syndrome. Dev Med Child Neurol 2011;53:658-63. 


\section{Supplementary data online published at:}

http://dx.doi.org/10.1016/j.seizure.2014.03.008.

Table Summary of studies using IEDs as outcome parameter in KD.

\begin{tabular}{|c|c|c|c|c|}
\hline $\begin{array}{l}\text { Author, year, } \\
\text { Study type }\end{array}$ & $\begin{array}{l}\text { No. of patients/age/ } \\
\text { No. of patients with } \\
\mathrm{IS}^{1} \text { or } \mathrm{LGS}^{2}\end{array}$ & \begin{tabular}{|l|} 
Definition of \\
responders/ \\
Responder rate \\
\end{tabular} & $\begin{array}{l}\text { Timing and type of } \\
\text { EEG/ } \\
\text { EEG analysis } \\
\end{array}$ & $\begin{array}{l}\text { Reported changes in } \\
\text { IEDs }^{3}\end{array}$ \\
\hline $\begin{array}{l}\text { Kang e.a. } \\
2005^{4} \\
\text { Retrospective } \\
\text { study }\end{array}$ & $\begin{array}{l}199 \text { children } \\
\text { mean age } 4.8 \\
\text { IS } n=39 \\
\text { LGS } n=75\end{array}$ & \begin{tabular}{|l}
$\geq 50 \%$ Sz reduction at \\
$3 \mathrm{~m} ., 6 \mathrm{~m}$ and $12 \mathrm{~m}$ \\
vs. baseline period \\
(duration not \\
mentioned) \\
Responder rate \\
$62 \%$ at $3 \mathrm{~m}$ \\
$58 \%$ at $6 \mathrm{~m}$ \\
$41 \%$ at $12 \mathrm{~m}$
\end{tabular} & $\begin{array}{l}\text { Routine awake EEG } \\
\text { somewhere between } \\
3 \text { and } 6 \mathrm{~m} \text {. in } 116 \\
\text { patients } \\
\text { Global review of } \\
\text { improvement }\end{array}$ & $\begin{array}{l}\text { Pts with frequent } \\
\text { generalized IEDs: } \\
\text { improvement in } \\
41 / 71 \\
\text { Pts with frequent } \\
\text { focal IEDs: } \\
\text { improvement in } \\
15 / 45 \\
\text { Correlation with } \\
\text { seizure frequency } \\
\text { was observed (but } \\
\text { not statistically } \\
\text { analyzed). }\end{array}$ \\
\hline $\begin{array}{l}\text { Hallböök e.a. } \\
2007^{1 \underline{6}} \\
\text { Prospective study }\end{array}$ & $\begin{array}{l}18 \text { children } \\
\text { mean age } 7.5 \\
\text { LGS } n=5\end{array}$ & $\begin{array}{l}\geq 50 \% \text { Sz reduction at } \\
3 \mathrm{~m} . \mathrm{vs} .3 \mathrm{~m} . \\
\text { baseline } \\
\text { Responder rate } 67 \%\end{array}$ & $\begin{array}{l}24 \text { h EEG at } 3 \text { m. } \\
\text { Automatic spike } \\
\text { detection }\end{array}$ & $\begin{array}{l}\text { At group level } \\
\text { significant reduction } \\
\text { of IEDs in sleep. } \\
\text { Correlation between } \\
\text { IED reduction and } \\
\text { clinical seizures } \\
\mathrm{R}=0,6\end{array}$ \\
\hline $\begin{array}{l}\text { Remahl e.a. } \\
2008^{\frac{17}{}} \\
\text { Prospective study }\end{array}$ & $\begin{array}{l}23 \text { children } \\
\text { mean age } 6.5 \\
\text { IS } n=1 \\
\text { LGS } n=4\end{array}$ & $\begin{array}{l}\geq 50 \% \text { Sz reduction at } \\
3 \mathrm{~m} . \mathrm{vs} .1 \mathrm{~m} . \\
\text { baseline } \\
\text { Responder rate } 70 \%\end{array}$ & $\begin{array}{l}24 \text { h EEG at } 3 \text { m. } \\
\text { Semi-quantitative } \\
\text { global review ( } 6 \\
\text { steps scale of } \\
\text { changes in IED } \\
\text { frequency) }\end{array}$ & $\begin{array}{l}\text { IED reduction in } 8 / 16 \\
\text { responders vs. 5/7 } \\
\text { non-responders (not } \\
\text { sign.) } \\
\text { No statements on } \\
\text { sleep EEG vs. awake } \\
\text { EEG }\end{array}$ \\
\hline $\begin{array}{l}\text { Dressler e.a. } \\
2010^{18} \\
\text { Retrospective } \\
\text { study }\end{array}$ & $\begin{array}{l}50 \text { children } \\
\text { mean age } 4.5\end{array}$ & $\begin{array}{l}\geq 50 \% \text { Sz reduction at } \\
6 \mathrm{~m} . \text { vs. } 2 \mathrm{~m} \text { baseline } \\
\text { Responder rate } 50 \%\end{array}$ & $\begin{array}{l}\text { routine awake EEG } \\
\text { between } 3 \text { and } 6 \mathrm{~m} \\
\text { Global review, of } \\
\text { disappearance of } \\
\text { IEDs }\end{array}$ & $\begin{array}{l}9 / 25 \text { responders } \\
\text { disappearance of } \\
\text { IEDs vs. } 1 / 24 \text { non- } \\
\text { responders }(p=0.009)\end{array}$ \\
\hline
\end{tabular}




\begin{tabular}{|c|c|c|c|c|}
\hline $\begin{array}{l}\text { Author, year, } \\
\text { Study type }\end{array}$ & $\begin{array}{l}\text { No. of patients/age/ } \\
\text { No. of patients with } \\
\mathrm{IS}^{1} \text { or } \mathrm{LGS}^{2}\end{array}$ & \begin{tabular}{|l} 
Definition of \\
responders/ \\
Responder rate \\
\end{tabular} & $\begin{array}{l}\text { Timing and type of } \\
\text { EEG/ } \\
\text { EEG analysis }\end{array}$ & $\begin{array}{l}\text { Reported changes in } \\
\text { IEDs }^{3}\end{array}$ \\
\hline \begin{tabular}{|l|} 
Kessler e.a. \\
$2011^{14}$ \\
Prospective study
\end{tabular} & $\begin{array}{l}37 \text { children } \\
\text { mean age } 5.7 \\
\text { LGS } n=2\end{array}$ & $\begin{array}{l}\geq 50 \% \text { Sz reduction at } \\
3 \mathrm{~m} \text {. vs. } 1 \mathrm{~m} \text {. baseline }\end{array}$ & $\begin{array}{l}30 \text { minute awake } \\
\text { EEG } \\
\text { at } 1 \mathrm{~m} \text {. and } 3 \mathrm{~m} . \\
\text { Visual count no. of } \\
\text { seconds with IEDs -> } \\
\text { IED index in \% }\end{array}$ & $\begin{array}{l}\text { individual spike index } \\
\text { decline of } \geq 10 \% \text { at } 1 \\
\mathrm{~m} \text {. in } 12 / 26 \\
\text { responders and in } \\
2 / 11 \text { non responders } \\
\geq 10 \% \text { reduction IED } \\
\text { index }>6 x \text { more } \\
\text { likely to be } \\
\text { responder }\end{array}$ \\
\hline $\begin{array}{l}\text { Li e.a. } \\
2013^{\underline{19}} \\
\text { Prospective } \\
\text { study }\end{array}$ & $\begin{array}{l}31 \text { children } \\
\text { mean age } 2.5 \\
\text { IS } n=16 \\
\text { LGS } n=11\end{array}$ & $\begin{array}{l}\geq 50 \% \text { Sz reduction at } \\
1 \text { and } 3 \mathrm{~m} . \text { vs. } 1 \mathrm{~m} \\
\text { baseline } \\
\text { Responder rate } \\
52 \% \text { at } 1 \mathrm{w} . \\
68 \% \text { at } 1 \mathrm{~m} . \\
71 \% \text { at } 3 \mathrm{~m} .\end{array}$ & $\begin{array}{l}\geq 12 \mathrm{~h} \text { video-EEG at } 1 \\
\text { w., } 1 \mathrm{~m} \text { and } 3 \mathrm{~m} \\
\text { Visual count no. of } \\
\text { seconds containing } \\
\text { IEDs in a selection of } \\
100 \text { seconds awake } \\
\text { EEG }\end{array}$ & $\begin{array}{l}\geq 50 \% \text { reduction of } \\
\text { index in } 5 / 31 \text { at } 1 \mathrm{w}, \\
14 / 31 \text { at } 1 \mathrm{~m} \text { and } \\
16 / 31 \text { at } 3 \mathrm{~m} . \\
\text { Correlation between } \\
\text { reduction of seizures } \\
\text { not mentioned }\end{array}$ \\
\hline
\end{tabular}

${ }^{1}$ IS= infantile spasms, ${ }^{2}$ LGS=Lennox Gastaut Syndrome, ${ }^{3}$ IED=interictal epileptic discharges. 


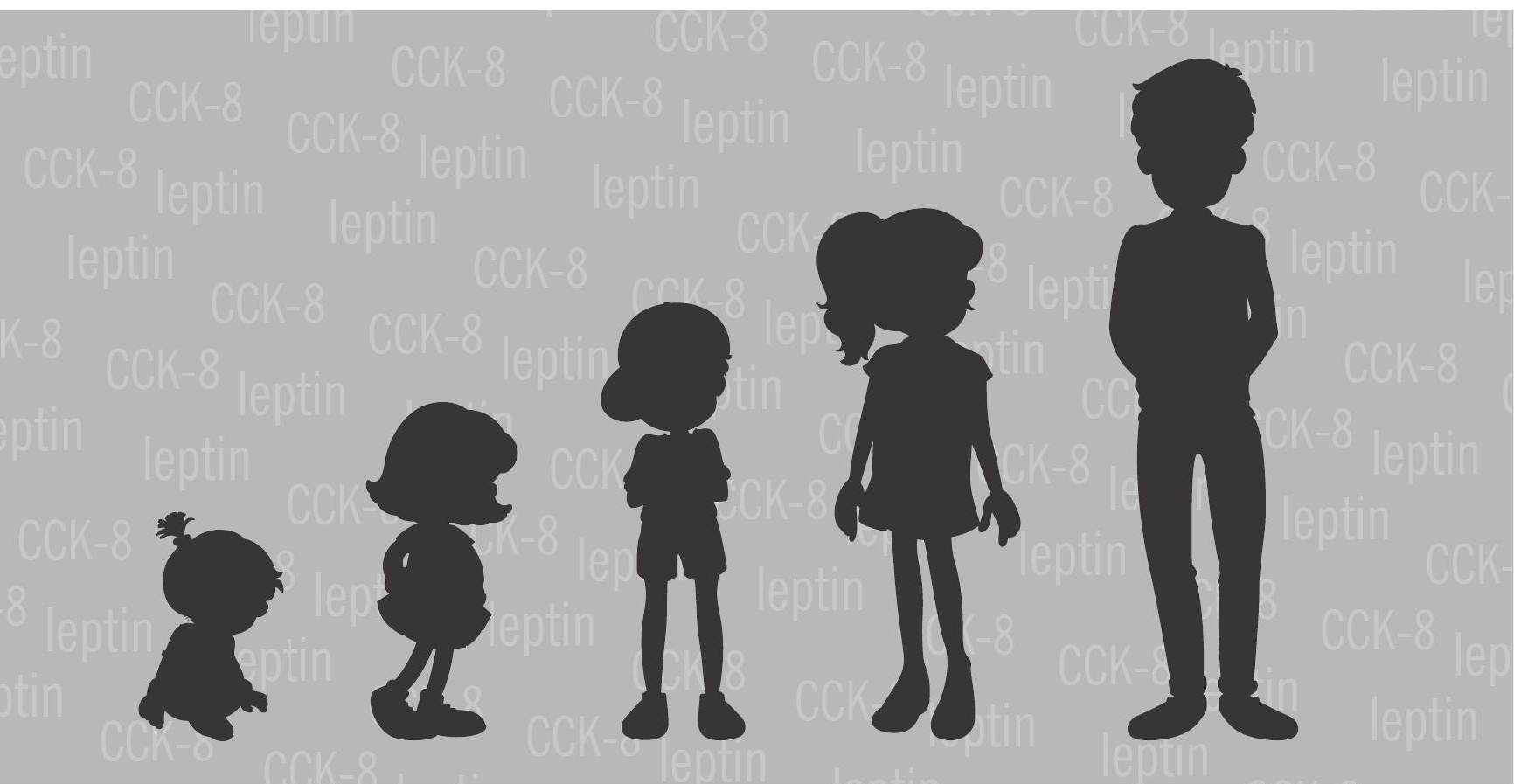




\section{Chapter 10}

A prospective study on changes in blood levels of cholecystokinin-8 and leptin in patients with refractory epilepsy treated with the ketogenic diet 


\section{Abstract}

\section{Objective}

To evaluate the changes in serum CCK-8 and leptin levels in patients with refractory epilepsy treated with the ketogenic diet (KD).

\section{Methods}

In this prospective study, patients aged between one and 40 years with refractory epilepsy were included. CCK- 8 and leptin were measured in serum at baseline and after three and 12 months of treatment with the KD using an enzyme-linked Immunoabsorbant Assay. Seizure frequency and responsiveness were calculated.

\section{Results}

Fifty-four patients were included; 26 patients (48\%) were still on the KD at 12 months. After three and 12 months, respectively, $39 \%$ and $26 \%$ were responders. CCK- 8 values were statistically significantly increased at three months $(p=0.005)$ and 12 months $(p=0.012)$. In responders this increase of CCK- 8 was significant $(p=0.020)$, whereas in the non-responders it was not $(p=0.34)$. Leptin values were decreased at three and 12 months, the decrease at three months being statistically significant in men $(p=0.028)$ and post-pubertal women $(p=0.007)$.

\section{Significance}

In responders to the $K D$, serum $C C K-8$ increased statistically significantly during treatment at three and 12 months. Serum leptin decreased statistically significantly at three months in men and in post-pubertal women. It is plausible that the increase of CCK- 8 and the decrease of leptin contribute to the anti-convulsive effect of the KD. 


\section{Introduction}

The ketogenic diet (KD) was developed in the 1920s to simulate the apparent anticonvulsant effects of the ketotic state induced by fasting. It is an individually calculated, high-fat and low-carbohydrate diet containing a sufficient quantity of proteins. Development of new anticonvulsant drugs resulted in a loss of interest in the KD. However, despite the use of different anti-epileptic drugs (AED), epilepsy remains refractory in approximately 30 percent of the patients. ${ }^{1}$ Hence, in the last few decades, there has been renewed interest in the KD, resulting in the introduction of various types of ketogenic diet alongside the classical KD. Many studies have since proved their efficacy, particularly in children, but also in adolescents and adults of different race, with different types of seizures and epilepsy syndromes. ${ }^{2-5}$

Many different theories have been suggested to explain the anti-epileptic effect of the $\mathrm{KD},{ }^{6,7}$ but the exact mechanisms are still unclear. Currently, the focus is on changes in energy metabolism. ${ }^{7,8}$ It is thought that these changes could have an influence on feeding and satiety centers, particularly in the hypothalamus. ${ }^{7,9}$ A variety of (neuro-) peptides may be altered, such as cholecystokinin-8 (CCK-8) and leptin, ${ }^{8,10}$ and this could have a significant influence on neuronal excitability and play a role in the anticonvulsive effect of the KD.

CCK is a hormone produced by endocrine cells of the small intestine. ${ }^{11,12}$ It is one of the brain-gut peptides found in the small intestine as well as in the central nervous system, existing in different molecular forms which vary in size from four to 83 amino acids. In humans, CCK- 58 is the most frequent variant, followed by CCK- 8 and CCK- 33 . In the brain, the main molecular form is CCK-8. ${ }^{12}$ Besides the gastrointestinal system, CCK-receptors are also present at peripheral nerve terminals of the myenteric and submucosal plexus and afferents of the vagus nerve. CCK is excreted after the consumption of a meal, mainly food rich in fat, especially long chain fatty acids, and proteins $^{11,12}$ and plays a role in satiety mechanisms, partly through stimulation of the afferent parts of the vagus nerve. Studies show that the excitatory response of CCK on the vagus nerve is amplified by the presence of leptin. ${ }^{13}$

In addition, CCK also plays a modulatory role in neuronal excitability ${ }^{14-16}$ and has anticonvulsive effects. ${ }^{17,18}$ Intraventricular or intraperitoneal administration of CCK-8 or a CCK-8 analogue in rodents suppresses seizures in different animal models for epilepsy. $^{19-22}$

Leptin is a hormone that is mainly produced in the white fat mass but also in much smaller amounts in the hypothalamus. ${ }^{23,24}$ It circulates in the blood plasma, the quantity being proportional to the amount of body fat. Leptin regulates energy balance, ${ }^{25}$ probably partly by its influence on the vagal nerve which expresses the leptin receptor and thereby affecting eating behavior and playing a major role in systemic energy regulation.

Leptin exerts modulatory effects on neuronal excitability and suppresses seizures. ${ }^{10,24}$ In different animal models for epilepsy, intracerebral, intranasal or intraperitoneal 
administration of leptin results in shorter and less frequent seizures. ${ }^{26}$ In juvenile rodents placed on a KD, there is a rise in serum leptin. ${ }^{25,27-29}$

Certain of these anti-convulsive effects of leptin and CCK- 8 might be mediated by the vagus nerve. ${ }^{17}$ Previous studies have shown the efficacy of vagal nerve stimulation (VNS) in reducing seizure frequency in adults ${ }^{30}$ and children. ${ }^{31}$

To date, there has been little information on whether the KD induces changes in the levels of CCK-8 and leptin in humans. We hypothesize that introduction of the KD causes a rise in CCK-8 and leptin concentration, an increase which might be related to seizure outcome.

This prospective study evaluates the possible changes in serum CCK-8 and leptin after the introduction of the KD in patients with refractory epilepsy.

\section{Materials and methods}

This clinical study was carried out at the epilepsy center Kempenhaeghe, Heeze, The Netherlands. Patients were included during the period January 2005 to October 2014. The study was approved by the Medical Ethics Committee and all patients and/or caregivers signed informed consent.

\section{Patients}

Patients aged between one and 40 years with refractory epilepsy were included. Refractory epilepsy was defined as seizures not adequately controlled by optimal treatment with $\geq 3$ AEDs. ${ }^{32}$ Patients were not eligible for epilepsy surgery.

Patients were excluded if they did not comply with the KD for at least three months or suffered from fatty acid oxidation disorders, metabolic disorders causing hypoglycemia or metabolic acidosis, hypertriglyceridemia, hypercholesterolemia, cardiovascular disorders, long QT syndrome, malignancies, progressive cerebral lesions, degenerative diseases, malnutrition, treatment with topiramate, acetazolamide or zonisamide combined with a positive family history of kidney stones or metabolic acidosis, or the expectation that compliance with the diet was not possible because of severe behavioral or motivational problems.

Because of the influence of sex and pubertal status on serum leptin, ${ }^{33}$ we subdivided patients into groups. Patients $\leq 11$ years old were considered pre-pubertal and patients $\geq 17$ years old post-pubertal. The treating pediatrician allocated the patients aged between 12 and 16 years old to the correct subgroup.

\section{Ketogenic diet}

The KD was introduced during a hospitalization of approximately one week. Usually the MCT-diet was applied, but on subject's request or if insurance did not refund the 
fat-emulsions of the MCT-diet, the classical diet was used. When only tube feeding was given, a liquid form of the classical KD was used. Changes could be made to the MCT-diet because of side-effects and other problems during the introduction phase.

The diet was introduced according to the Dutch guideline for KD, ${ }^{34}$ which does not include a prior fasting period. In the classical diet, the fat-intake was gradually built up over four to five days. In the MCT-diet, it took two to three weeks to build up the fatintake to the required ratio. The building schedule started with ten grams of MCT- fat, a quantity which was slowly increased to the calculated amount. In addition, the LCTfat was increased in four days to the calculated amount of fat and then decreased in small steps equivalent to the MCT-fat increase. Vitamin and mineral supplements were administered to avoid deficiencies.

Because of the growing amount of evidence of improved tolerability of the modified Atkins diet (MAD), all adult patients who started with the KD after 2010 received the MAD on an outpatient basis. Carbohydrates were restricted to ten $\mathrm{g} /$ day and the intake of fat and protein was actively encouraged. Clear carbohydrate-free fluids and calorie intake were not restricted. After three months, when adequate ketosis was reached or to prevent weight loss, carbohydrates were gradually added to the diet in five gram steps with a maximum of $20 \mathrm{~g} /$ day.

The start of the diet was defined as the first change made to the daily nutrition of the patient. The end of the diet was defined as the first step in down titration to a regular diet. As is usual in everyday practice, the KD was tapered off if there was no positive response after three to six months of treatment. In the case of a positive response, the KD could be continued for two years. ${ }^{32}$

\section{Evaluation}

Patients were treated by a specialized multidisciplinary team. Evaluation was carried out at baseline, six weeks after diet initiation and subsequently every three months, and serum blood levels of leptin and CCK-8 were measured.

Patients and/or caregivers were asked to record seizures continuously in a diary, starting one month before KD introduction. The mean seizure frequency during the follow-up period was calculated each month prior to the laboratory tests and compared to the baseline mean seizure frequency. When the mean seizure frequency was reduced by $\geq 50 \%$, the patient was defined as a responder. An increase was defined as a rise of $\geq 50 \%$. If seizure diaries were incomplete, the treating physician was asked to define the patient as a responder or non-responder, using patient's and/or caregiver's information for qualitative data analysis. A seizure cluster $(\geq 5$ seizures in a short time) was calculated as one seizure for the seizure frequency calculations. Intention to treat (ITT) analysis was performed. 


\section{Laboratory tests}

At baseline, three and 12 months after introduction of the KD, CCK- 8 and leptin were measured in blood serum. Blood samples were collected in the morning after an overnight fast. CCK-8 was measured by enzyme-linked Immunoabsorbant assay (Cloud-Clone Corp., Houston, Texas). This is a competitive assay specific for human CCK-8. Measurements were performed according to manufacturer's instructions, with the exception of the first incubation, which was performed at 37 degrees Celsius for two hours. Leptin was measured in serum using a quantitative sandwich enzyme immunoassay (Quantakine ${ }^{\circledR}$ ELISA, R\&D Systems, Abingdon, UK), according to the manufacturer's instructions. Results were calculated using a leptin standard curve, limit of quantification was $0.1 \mu \mathrm{g} / \mathrm{l}$.

\section{Statistics}

Statistical analysis was performed using SPSS 22.0 for Windows. We used the 'relatedsamples Wilcoxon signed rank test' and the paired $t$-test to compare results within groups, the 'independent Mann-Withney $U$ test' to compare results between groups and 'Pearson correlations' to evaluate correlations between changes of CCK-8 and leptin.

\section{Results}

\section{Patient characteristics}

Table 10.1 summarizes the main demographic and clinical characteristics at baseline. Fifty-four patients with refractory epilepsy started the KD. Four patients had previously been treated with VNS, one patient was treated during the study. The MCTdiet was replaced by a classical diet in one patient and in three patients, the mixed variant of MCT and the classical diet were replaced by the classical KD.

\section{Seizure frequency}

Seizure outcome is reported in Table 10.2. Information on 54 patients was obtained. At 12 months, 26 patients (48\%) were still on the KD.

Data were quantitatively analyzed in 47 patients at three months and in 23 patients at 12 months.

There was a statistically significant decrease in the mean seizure frequency at three and 12 months from 143 to $61(p=0.033)$ and $58(p=0.014)$, respectively. 
Table 10.1 Baseline demographic and clinical characteristics.

\begin{tabular}{|c|c|c|c|c|}
\hline & Mean & Min-max & $\mathrm{N}=54$ & $(\%)$ \\
\hline \multicolumn{5}{|l|}{ Gender } \\
\hline Male & & & 25 & 46 \\
\hline Female & & & 29 & 54 \\
\hline \multicolumn{5}{|l|}{ Age (years) } \\
\hline At seizure onset & 14 & 0.1-39 & & \\
\hline At KD initiation & 4 & $0.9-20$ & & \\
\hline Duration of epilepsy (years) & 10 & $0.9-35$ & & \\
\hline Number of AEDs at KD initiation & 2.7 & $1-6$ & & \\
\hline Poly-therapy at KD initiation & & & 47 & 87 \\
\hline VNS treatment & & & 5 & 9 \\
\hline Seizure types & 3.5 & $1-7$ & & \\
\hline Absences & & & 25 & 46 \\
\hline Atonic & & & 11 & 20 \\
\hline Myoclonic & & & 27 & 50 \\
\hline Clonic & & & 14 & 26 \\
\hline Tonic & & & 30 & 56 \\
\hline Tonic clonic & & & 25 & 46 \\
\hline Epileptic spasm & & & 4 & 7 \\
\hline Focal with impaired consciousness & & & 21 & 39 \\
\hline Focal without impaired consciousness & & & 8 & 15 \\
\hline \multicolumn{5}{|l|}{ Syndrome classification } \\
\hline Benign childhood epilepsy with centro-temporal spike & & & 1 & 2 \\
\hline Eyelid Myoclonia with absences & & & 1 & 2 \\
\hline Childhood absence epilepsy & & & 1 & 2 \\
\hline Epilepsy with myoclonic absences & & & 1 & 2 \\
\hline Juvenile myoclonus epilepsy & & & 1 & 2 \\
\hline West syndrome & & & 4 & 7 \\
\hline Lennox-Gastaut syndrome & & & 3 & 6 \\
\hline Doose syndrome & & & 3 & 6 \\
\hline Dravet syndrome & & & 4 & 7 \\
\hline GLUT-1-deficiency syndrome & & & 1 & 2 \\
\hline Generalized epilepsies & & & 6 & 11 \\
\hline Localisation-related epilepsies & & & 28 & 51 \\
\hline \multicolumn{5}{|l|}{ Diet type at initiation KD } \\
\hline Classical & & & 9 & 17 \\
\hline MCT & & & 28 & 50 \\
\hline Mixed & & & 8 & 16 \\
\hline MAD & & & 9 & 17 \\
\hline \multicolumn{5}{|l|}{ Diet type at 12 months KD ( $\mathrm{N}=26)$} \\
\hline Classical & & & 13 & 50 \\
\hline MCT & & & 8 & 30 \\
\hline Mixed & & & 2 & 8 \\
\hline MAD & & & 3 & 12 \\
\hline
\end{tabular}

$\mathrm{N}$, number; Min-Max, minimum-maximum; KD, ketogenic diet; VNS, vagus nerve stimulator; AED, antiepileptic drug; MAD, modified Atkins diet. 
Table 10.2 Number of responders KD in ITT analysis.

\begin{tabular}{llrlrl}
\hline & & \multicolumn{2}{c}{3 months } & \multicolumn{2}{c}{12 months } \\
& & $\mathrm{N}$ & $(\%)$ & $\mathrm{N}$ & $(\%)$ \\
\hline ITT N=54 & Responders & 21 & $(38.9)$ & 14 & $(25.9)$ \\
& seizure free & 2 & $(3.7)$ & 4 & $(7.4)$ \\
& $>90 \%$ & 3 & $(5.6)$ & 4 & $(7.4)$ \\
& $>50 \%$ & 16 & $(29.6)$ & 6 & $(11.1)$ \\
\hline
\end{tabular}

$\mathrm{ITT}$, intention to treat; $\mathrm{N}$, number; $\mathrm{KD}$, ketogenic diet.

\section{CCK-8}

CCK-8 values of the 26 patients with 12 months' follow-up are presented in Figure 10.1 and Table 10.3.

At three and 12 months, values of one, respectively, three patients were missing due to not fasting or too small serum sample volumes. During the KD there is an increase in serum CCK-8 values which is statistically significant at three months $(p=0.005)$ and at 12 months $(p=0.012)$. The increase between three and 12 months is nearly significant $(p=0.055)$.

In the total patient population ( $N=54)$, the mean CCK-8 value at three months' followup is statistically significantly increased in the responder group $(p=0.020)$ but not in the non-responder group $(p=0.34)$.

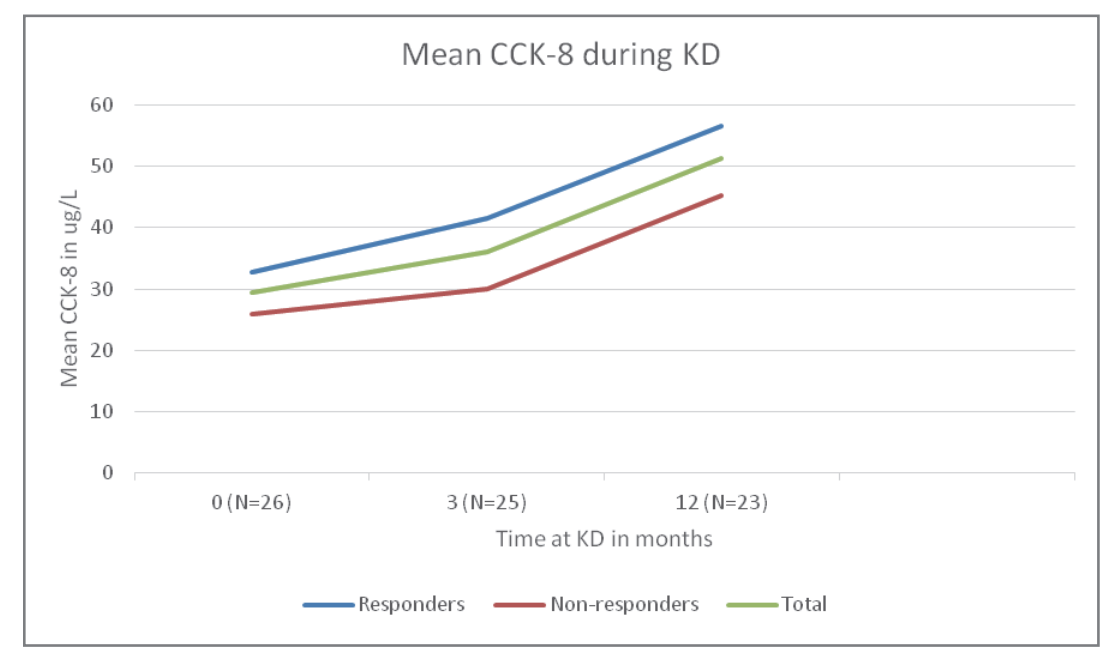

Figure 10.1 Mean CCK-8 values at baseline, three and 12 months on the KD. 
Table 10.3 Mean CCK-8 values at baseline, three and 12 months on the KD.

\begin{tabular}{lccc}
\hline & Baseline N=26 & 3 months N=25 & 12 months N=23 \\
\hline Responders & 32,79 & 41,56 & 56,45 \\
Non-responders & 25,94 & 30,04 & 45,29 \\
Total population with 12 months follow-up & 29,35 & 35,98 & 51,31 \\
\hline
\end{tabular}

CCK-8 in ug/l; $\mathrm{N}$, number.

\section{Leptin}

The course of leptin is evaluated in all patients $(\mathrm{N}=54)$. Patients were subdivided into four groups according to sex and pre- or post-pubertal status. Data are shown in Figure 10.2 and Table 10.4. At 12 months, nine men (six pre-pubertal and three postpubertal) were treated with the KD; in women, the numbers were seven and four, respectively. There is a decrease in serum leptin in all subgroups at three and 12 months' follow-up. This decrease is statistically significant at three months' followup in post-pubertal men $(p=0.028)$ and post-pubertal women $(p=0.007)$. Because of the small sample sizes it was not possible to make a statistically correct evaluation of leptin decrease at 12 months and a comparison of the results of the mean serum leptin in responders and non-responders.

There were no significant correlations between the increase in CCK-8 and the decrease in leptin values.

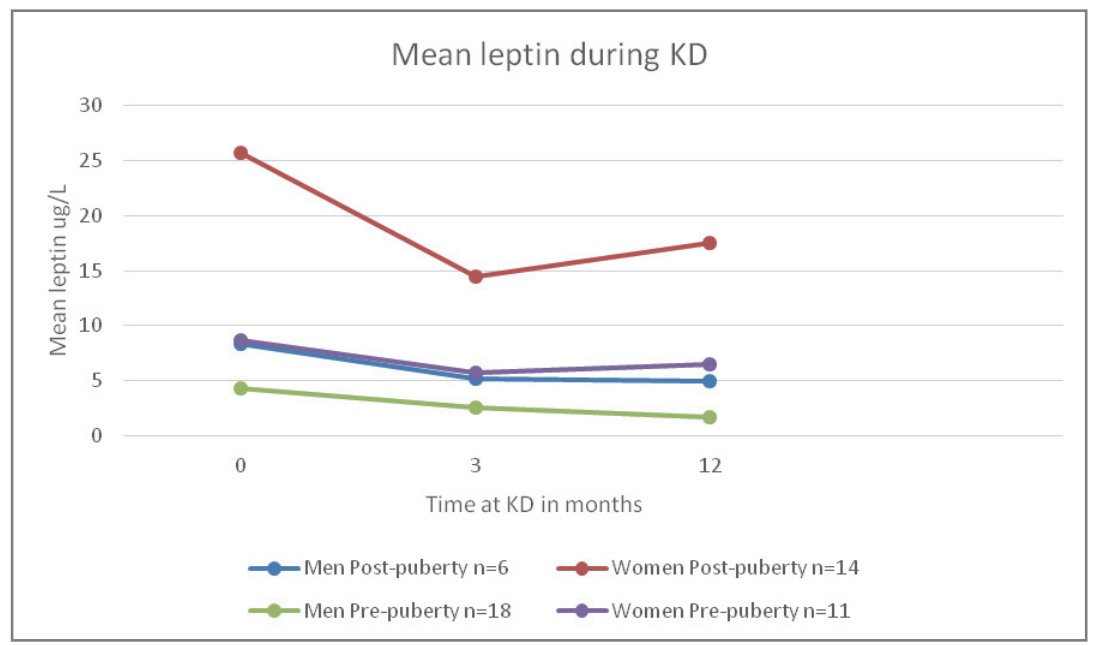

Figure 10.2 Mean leptin levels at baseline, three and 12 months on the KD. 
Table 10.4 Mean leptin levels at baseline, three and 12 months on the KD.

\begin{tabular}{lccc}
\hline & Baseline & 3 months & 12 months \\
\hline Men Pre-puberty $(\mathrm{N}=18)$ & 4,3 & 2,6 & 1,7 \\
Men Post-puberty $(\mathrm{N}=6)$ & 8,4 & 5,2 & 5,0 \\
Women Pre-puberty $(\mathrm{N}=11)$ & 8,7 & 5,7 & 6,5 \\
Women Post-puberty $(\mathrm{N}=14)$ & 25,7 & 14,5 & 17,5 \\
\hline
\end{tabular}

Mean leptin in ug/l; N, Number.

\section{Discussion}

This prospective study evaluates the changes in serum CCK-8 and leptin after the introduction of the KD in patients with refractory epilepsy.

The response rate of the KD was $39 \%$ and $26 \%$ at three and 12 months' follow-up, respectively. This is in line with the literature. In a recent Cochrane review, authors concluded that despite the heterogeneity, all trials showed that at least $38 \%$ of the patients had a $50 \%$ reduction in seizure frequency compared to controls at three months, and this response was maintained for up to a year. ${ }^{5}$

In the present study, CCK- 8 increases during the KD and this increase is statistically significant in responders, whereas it is not in the non-responders. As far as we are aware, there have been no studies on blood CCK- 8 levels in humans while on the KD. In some articles, it is presumed that the serum concentration of CCK- $8^{8}$ and leptin ${ }^{25}$ in people on the KD would be higher compared to those on a normal diet. Since CCK is mainly excreted when food rich in fat is consumed and leptin concentrations increase when fat mass increases due to the KD. It has been demonstrated that consumption of food with a high fat concentration activates the vagal nerve and that this process is mediated by the secretion of CCK. ${ }^{17,35}$ Bray et al. showed that intraperitoneal administration of 3-hydroxy butyrate acid, a product of fatty acid oxidation, reduces food intake in rats, but solely in the case of a normally functioning vagal nerve. ${ }^{36}$ While the patient is on the KD, the vagal nerve may be additionally stimulated because of the large degree of fatty acid oxidation. Peters et al. suggested that the action of CCK and leptin on sensitive vagal nerve afferents may not be limited to controlling food intake alone. ${ }^{13}$ Certain anti-convulsive effects of CCK- 8 and leptin could be mediated by the vagal nerve, ${ }^{17}$ because vagal nerve stimulation reduces seizure frequency. ${ }^{37}$

The anti-convulsive action of CCK has also been proven in various animal studies. Intracerebroventricular administration of CCK-8 in rats and intraperitoneal administration in mice and rats diminished seizures induced by different provoking mechanisms in epilepsy models. ${ }^{19-21,38}$ CCK-8 also amplified the anti-convulsive effect of the AED vigabatrine in a rat model for human partial epilepsy. ${ }^{39}$

In this study, we found that leptin decreased during treatment with the KD, while we had hypothesized an increase. At first, these results seemed surprising because in 
rodents placed on a KD, there was a rise in leptin. ${ }^{27,28}$ Furthermore, some studies describing the possible mechanisms of actions of the KD suggest there would also be an increase in leptin in humans on the KD, and that this, in part, could be responsible for its anti-epileptic effects. ${ }^{10,25,40}$ Stafstrom et al., however, suggested that because of the mimicked starvation and the altered energy metabolism, with signals of shortage of energy due to ketosis and low glucose-levels, there would be a decrease in leptin (and insulin) in humans on the KD. ${ }^{41}$ As a result of this decrease in leptin and insulin, there would be an increase in the peptides stimulating food intake as neuropeptide-Y (NPY). NPY is one of the most abundant neuropeptides in the nervous system. It regulates food intake by increasing meal sizes and diminishing meal latency. NPY is also anti-convulsive and inhibits excessive neuronal excitability. ${ }^{42}$ And, in the absence of leptin, hypothalamic expression of NPY is increased. ${ }^{25}$ Kinzig showed an increase in mRNA expression of NPY in the hypothalamus in rats on the KD. ${ }^{43}$ Weinshenker ${ }^{44}$ suggested the decrease in leptin but didn't find any changes in expression of NPY in mice on the KD. The course of NPY in humans using the KD still remains unclear. The decrease in leptin in humans on the KD has been confirmed in a study of patients with rheumatoid arthritis using the KD. ${ }^{45}$ Also, Giordano ${ }^{8}$ stated that in cases consuming a $K D$, there would be a decrease in leptin with an increase in NPY and CCK.

A limitation of this study is the relatively small sample size which makes it impossible to compare the leptin values between responders and non-responders. Only patients with a minimum of three months' follow-up were included, resulting in a higher responder rate. The purpose of this study was, however, to determine the course of CCK-8 and leptin during treatment with the KD.

The added value of the present study is that it is the first to evaluate the course of CCK-8 and leptin in humans with refractory epilepsy treated with the KD.

\section{Conclusion}

In conclusion, during treatment with the KD, serum CCK-8 increases statistically significantly at three and 12 months, with a statistically significantly higher increase in responders. Serum leptin decreases statistically significantly at three months. Further research is necessary to evaluate the relationship between these changes in neuropeptides and the anti-convulsive effects of the KD. 


\section{References}

1. Sander JW. Some aspects of prognosis in the epilepsies: a review. Epilepsia I1993;34: 1007-16.

2. Freeman JM, Vining EP, Pillas DJ, Pyzik PL, Casey JC, Kelly LM. The efficacy of the ketogenic diet-1998: a prospective evaluation of intervention in 150 children. Pediatrics I1998;102: 1358-63.

3. Neal EG, Chaffe H, Schwartz RH, Lawson MS, Edwards N, Fitzsimmons G, Whitney A, Cross JH. The ketogenic diet for the treatment of childhood epilepsy: a randomised controlled trial. Lancet Neurol 12008;7: 500-6.

4. Lambrechts DA, Wielders LH, Aldenkamp AP, Kessels FG, de Kinderen RJ, Majoie MJ. The ketogenic diet as a treatment option in adults with chronic refractory epilepsy: efficacy and tolerability in clinical practice. Epilepsy Behav 12012;23: 310-4.

5. Levy RG, Cooper PN, Giri P. Ketogenic diet and other dietary treatments for epilepsy. Cochrane Database Syst Rev 12012;3: CD001903.

6. Freeman J, Veggiotti P, Lanzi G, Tagliabue A, Perucca E, Institute of Neurology ICMF. The ketogenic diet: from molecular mechanisms to clinical effects. Epilepsy Res 12006;68: 145-80.

7. Schwartzkroin PA. Mechanisms underlying the anti-epileptic efficacy of the ketogenic diet. Epilepsy Res I1999;37: 171-80.

8. Giordano C, Marchio M, Timofeeva E, Biagini G. Neuroactive peptides as putative mediators of antiepileptic ketogenic diets. Front Neurol I2014;5: 63.

9. Kinzig KP, Hargrave SL, Hyun J, Moran TH. Energy balance and hypothalamic effects of a highprotein/low-carbohydrate diet. Physiol Behav 12007;92: 454-60.

10. Rho JM, Stafstrom CE. The ketogenic diet: what has science taught us? Epilepsy Res I2012;100: 210-7.

11. Liddle RA. Cholecystokinin cells. Annu Rev Physiol I1997;59: 221-42.

12. Crawley JN, Corwin RL. Biological actions of cholecystokinin. Peptides I1994;15: 731-55.

13. Peters JH, Simasko SM, Ritter RC. Modulation of vagal afferent excitation and reduction of food intake by leptin and cholecystokinin. Physiol Behav 12006;89: 477-85.

14. Ghijsen WE, Leenders AG, Wiegant VM. Regulation of cholecystokinin release from central nerve terminals. Peptides 12001;22: 1213-21.

15. Fuxe K, Li XM, Bjelke B, Hedlund PB, Biagini G, Agnati LF. Possible mechanisms for the powerful actions of neuropeptides. Ann N Y Acad Sci 11994;739: 42-59.

16. Gall C. Seizures induce dramatic and distinctly different changes in enkephalin, dynorphin, and CCK immunoreactivities in mouse hippocampal mossy fibers. J Neurosci I1988;8: 1852-62.

17. Nylen K, Likhodii S, Burnham WM. The ketogenic diet: proposed mechanisms of action. Neurotherapeutics 12009;6: 402-5.

18. Xi-Ru W, Zhao-Hui W, Wai-Song S, Wei-Lan L, Bao-Qiang Y, Hong P, John-Bao Z. Molecular neurobiological approach to the pathogenesis of epilepsy: a preliminary study. Brain Dev I1995;17 Suppl: 73-6.

19. Kadar T, Pesti A, Penke B, Telegdy G. Inhibition of seizures induced by picrotoxin and electroshock by cholecystokinin octapeptides and their fragments in rats after intracerebroventricular administration. Neuropharmacology I1984;23: 955-61.

20. Kadar T, Borda L, Zarandi M, Penke B, Kovacs K, Telegdy G. Inhibition of electroshock-induced seizures by cholecystokinin-related peptides in mice. Acta Physiol Hung 11987;69: 43-50.

21. Zhang LX, Zhou Y, Du Y, Han JS. Effect of CCK-8 on audiogenic epileptic seizure in P77PMC rats. Neuropeptides I1993;25: 73-6.

22. Yamamoto $Y$, Itano T, Miyamoto O, Tokuda M, Matsui H, Janjua NA, Suwaki H, Okada $Y$, Negi T, Murakami TH, et al. Effect of ceruletide on epileptogenesis in amygdaloid kindled rats. Brain Res I1993;630: 353-6.

23. Harvey J. Leptin: a multifaceted hormone in the central nervous system. Mol Neurobiol I2003;28: 24558.

24. Harvey J. Leptin: a diverse regulator of neuronal function. J Neurochem I2007;100: 307-13.

25. Thio LL. Hypothalamic hormones and metabolism. Epilepsy Res 12012;100: 245-51.

26. Erbayat-Altay E, Yamada KA, Wong M, Thio LL. Increased severity of pentylenetetrazol induced seizures in leptin deficient ob/ob mice. Neurosci Lett 12008;433: 82-6. 
27. Thio LL, Erbayat-Altay E, Rensing N, Yamada KA. Leptin contributes to slower weight gain in juvenile rodents on a ketogenic diet. Pediatr Res I2006;60: 413-7.

28. Kinzig KP, Honors MA, Hargrave SL, Davenport BM, Strader AD, Wendt D. Sensitivity to the anorectic effects of leptin is retained in rats maintained on a ketogenic diet despite increased adiposity. Neuroendocrinology 12010;92: 100-11.

29. Honors MA, Davenport BM, Kinzig KP. Effects of consuming a high carbohydrate diet after eight weeks of exposure to a ketogenic diet. Nutr Metab (Lond) I2009;6: 46.

30. Handforth A, DeGiorgio CM, Schachter SC, Uthman BM, Naritoku DK, Tecoma ES, Henry TR, Collins SD, Vaughn BV, Gilmartin RC, Labar DR, Morris GL, 3rd, Salinsky MC, Osorio I, Ristanovic RK, Labiner DM, Jones JC, Murphy JV, Ney GC, Wheless JW. Vagus nerve stimulation therapy for partial-onset seizures: a randomized active-control trial. Neurology 11998;51: 48-55.

31. Klinkenberg S, Aalbers MW, Vles JS, Cornips EM, Rijkers K, Leenen L, Kessels FG, Aldenkamp AP, Majoie M. Vagus nerve stimulation in children with intractable epilepsy: a randomized controlled trial. Dev Med Child Neurol I2012;54: 855-61.

32. Kossoff EH, Zupec-Kania BA, Amark PE, Ballaban-Gil KR, Christina Bergqvist AG, Blackford R, Buchhalter JR, Caraballo RH, Helen Cross J, Dahlin MG, Donner EJ, Klepper J, Jehle RS, Kim HD, Christiana Liu YM, Nation J, Nordli DR, Jr., Pfeifer HH, Rho JM, Stafstrom CE, Thiele EA, Turner Z, Wirrell EC, Wheless JW, Veggiotti P, Vining EP, Charlie Foundation PCotCNS, Practice Committee of the Child Neurology S, International Ketogenic Diet Study G. Optimal clinical management of children receiving the ketogenic diet: recommendations of the International Ketogenic Diet Study Group. Epilepsia I2009;50: 304-17.

33. Ganji V, Kafai MR, McCarthy E. Serum leptin concentrations are not related to dietary patterns but are related to sex, age, body mass index, serum triacylglycerol, serum insulin, and plasma glucose in the US population. Nutr Metab (Lond) I2009;6: 3.

34. Van den Hurk TAM, van der Louw EJTM. Dieetbehandelingsrichtlijn ketogeen dieet voor kinderen (018 jaar) met refractaire epilepsie. Evidence-based handleiding voor een multidisciplinaire behandeling. Utrecht: UMC Utrecht; 2007.

35. Luyer MD, Greve JW, Hadfoune M, Jacobs JA, Dejong CH, Buurman WA. Nutritional stimulation of cholecystokinin receptors inhibits inflammation via the vagus nerve. J Exp Med I2005;202: 1023-9.

36. Bray GA. Afferent signals regulating food intake. Proc Nutr Soc 12000;59: 373-84.

37. The Vagus Nerve Stimulation Study Group. A randomized controlled trial of chronic vagus nerve stimulation for treatment of medically intractable seizures. . Neurology 11995;45: 224-30.

38. Kadar T, Pesti A, Penke B, Toth G, Zarandi M, Telegdy G. Structure-activity and dose-effect relationships of the antagonism of picrotoxin-induced seizures by cholecystokinin, fragments and analogues of cholecystokinin in mice. Neuropharmacology I1983;22: 1223-9.

39. Ferraro G, Sardo P. Cholecystokinin-8 sulfate modulates the anticonvulsant efficacy of vigabatrin in an experimental model of partial complex epilepsy in the rat. Epilepsia 12009;50: 721-30.

40. Rho JM, Sankar R. The ketogenic diet in a pill: is this possible? Epilepsia I2008;49 Suppl 8: 127-33.

41. Stafstrom CE. In: Epilepsy and the ketogenic diet. Totowa, New Yersey: Humana Press; 2010, p. 279287.

42. Clynen E, Swijsen A, Raijmakers M, Hoogland G, Rigo JM. Neuropeptides as Targets for the Development of Anticonvulsant Drugs. Mol Neurobiol 12014.

43. Kinzig KP, Scott KA, Hyun J, Bi S, Moran TH. Altered hypothalamic signaling and responses to food deprivation in rats fed a low-carbohydrate diet. Obes Res 12005;13: 1672-82.

44. Weinshenker D. The contribution of norepinephrine and orexigenic neuropeptides to the anticonvulsant effect of the ketogenic diet. Epilepsia 12008;49 Suppl 8: 104-7.

45. Fraser DA, Thoen J, Bondhus S, Haugen M, Reseland JE, Djoseland O, Forre O, Kjeldsen-Kragh J. Reduction in serum leptin and IGF-1 but preserved T-lymphocyte numbers and activation after a ketogenic diet in rheumatoid arthritis patients. Clin Exp Rheumatol I2000;18: 209-14. 


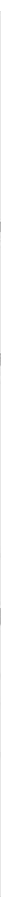




\section{Chapter 11}

\section{General discussion}

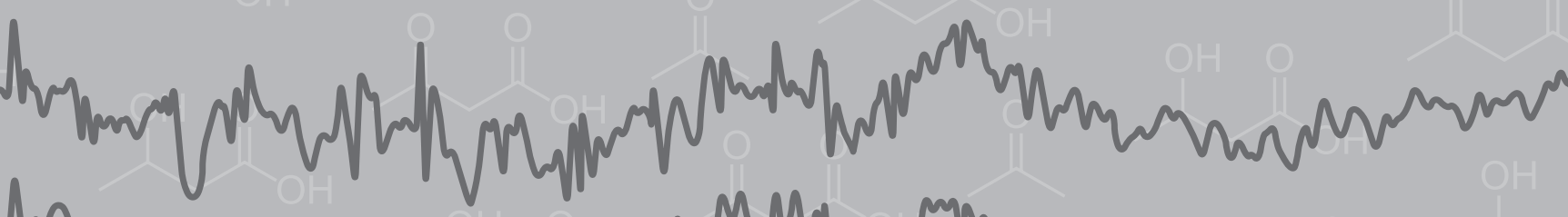

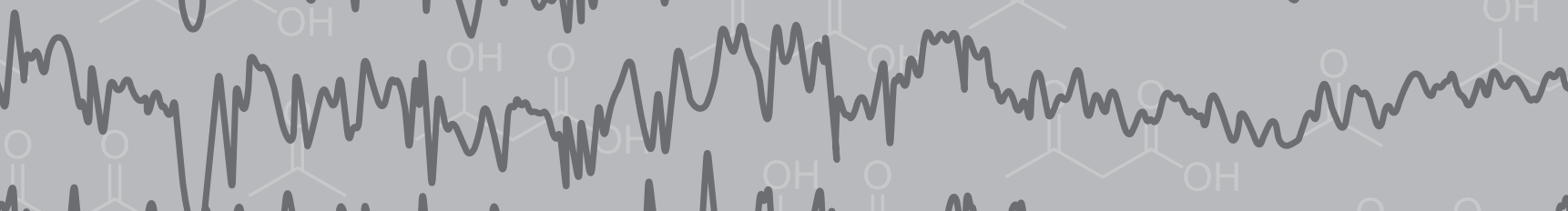

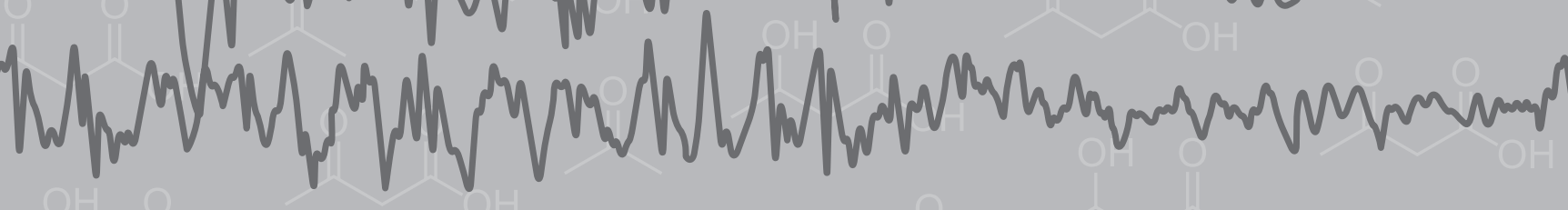


Chapter 11

11 


\section{General discussion}

The studies described in this thesis aimed to answer the following main questions:

1. Is the ketogenic diet (KD), especially the MCT-diet, an effective and well-tolerated treatment for children, adolescents and adults with refractory epilepsy?

2. Are electro-encephalography (EEG) and specific laboratory investigations useful during treatment with the KD?

3. Is the ketogenic diet a cost-effective treatment for children with refractory epilepsy?

The question that emerges is: what is the position of the KD as compared to other treatments for patients with refractory epilepsy?

Finally some recommendations and suggestions for future research are summarized.

\section{Is the ketogenic diet, especially the MCT-diet an effective and well tolerated treatment for children, adolescents and adults with refractory epilepsy?}

\section{Ketogenic diet in children}

The Cochrane review, published in 2003 , concluded that there is no reliable evidence from randomized controlled trials (RCT) to support the use of ketogenic diets for people with epilepsy. There are a number of larger observational studies of ketogenic diets, five of which are prospective. Studies report on KD's efficacy with recognized short-term side-effects, but longer-term effects (longer than 12 months) have not been addressed. Some authors comment on improved cognition and behavior, but, apart from one ongoing study, there has been no formal use of validated quality of life (QOL) or cognitive measures. ${ }^{1}$

The Cochrane review, published in 2012, included four non-blinded randomized studies of the ketogenic diet. Authors concluded that, despite the heterogeneity, all trials showed that at least $38 \%$ of the patients had a $50 \%$ reduction in seizure frequency compared to controls at three months, and this response was maintained for up to a year. Studies suggest that in children, the ketogenic diet results in short- to medium-term benefits in seizure control, the effects of which are comparable to those achieved with modern antiepileptic drugs. Follow-up periods of at least 12 months and preferably longer are advised to assess retention and long-term adverse effects. ${ }^{2}$ The RCT by Neal et al. showed that the classical diet did not have any advantage over MCT-diet in terms of efficacy and tolerability. ${ }^{3}$

Until now, prospective long-term data in children, treated with the ketogenic diet, with a follow-up of at least 2 years, are rare ${ }^{4}$, are mostly about classical KD, or focused on specific side-effects. ${ }^{5-7}$ 
One of the major challenges in clinical trials is to define the most appropriate outcome measures. All conventional outcome measures have limitations. The most striking example, discovered during the cost-effectiveness study, was the story of an adolescent whose major seizures ceased, but the frequency of minor seizure (with only a twitch in the face with unimpaired consciousness) increased dramatically. The patient and his parents were very satisfied with the results of the treatment, and now, over two years later, are still willing to continue the KD. Overall, however, an adverse outcome in responder rate was found, defined as $\geq 50 \%$ seizure reduction as well as in percent seizure change.

The measurement of responder rate, defined as $\geq 50 \%$ seizure reduction, is well established in trials on epilepsy treatment. It was introduced in antiepileptic drug clinical trials as a requirement by European and other health authorities and has similar advantages and disadvantages as percent seizure reduction. $A \geq 50 \%$ reduction may not reflect functional or QOL improvements; some argue that it does not adequately forecast real-world drug performance. Most patients with a $50 \%$ reduction in seizures are still unable to resume their daily activities and, therefore, remain unsatisfied. Trials that incorporate both QOL and responder rate assessments noted QOL improvements with treatment, but correlations between responder rates and QOL were not investigated. ${ }^{8}$

To obtain more data on the long-term efficacy and safety of the KD in children and adolescents, a prospective observational study was conducted. In an attempt to introduce a broader approach to measuring efficacy and tolerability, changes in seizure severity and frequency of seizure clusters were added as outcome parameters to the responder rate defined as $\geq 50 \%$ seizure reduction and percentage seizure reduction. Psychological assessment was added to laboratory measurements, the performance of ECGs and parental interviews to evaluate possible adverse effects of the KD. Overall benefits of the KD in clinical practice were evaluated using retention rates, a measure which expresses the willingness of a patient to continue the diet. It is considered to be a composite of diet efficacy and tolerability and is, therefore, an important clinical parameter. ${ }^{9}$ At the start of our RCT there was only one RCT randomising KD with care as usual and, to our knowledge, there was no information about the cost-effectiveness of the KD. We, therefore, initiated a RCT, designed as a cost-effectiveness trial.

\section{Efficacy}

Forty-eight children with refractory epilepsy were included in the observational KD study. Thirty-eight were treated with the MCT-diet. Highest responder rate, in terms of more than $50 \%$ seizure reduction (i.e. $22.9 \%$ ), established using an intention-totreat (ITT) analysis, was seen at six and nine months of treatment. At other moments it was $16.7 \%$. 
After three months' treatment, nine of the fifteen patients (60\%) with seizure clusters during baseline had $\geq 50 \%$ reduction in seizure clusters. Of these nine responders, seven patients were not responders for the total seizure frequency. From three months of treatment onwards, most of the patients on the KD experienced a relevant decrease in seizure severity, mainly related to the most severe seizure type.

In the RCT, forty-eight children were included in the analyses (twenty-six in the KD group, twenty-two in the care as usual (CAU) group). Based on an ITT analysis at four months, $50 \%$ of the participants in the KD group had a seizure reduction of $\geq 50 \%$ from baseline, compared with $18.2 \%$ of the participants in the CAU group. Mean seizure frequency at four months compared with baseline was, after removal of two outliers in the KD group, significantly lower in the KD group than in the CAU group.

Results on seizure severity were comparable to the outcome in the observational study.

At 6 weeks, 3 times as many patients using the KD had a relevant decrease in seizure severity score, compared with the patients in the CAU group. At 4 months' treatment, there were twice as many patients in the KD group reporting a relevant decrease in seizure severity score. Differences between the two groups were statistically significant at 6 weeks and at 4 months; a trend could be detected. Except for one child of the KD group, changes in seizure severity were related to the most severe seizure type.

The efficacy of our RCT is in line with the RCTs of Neal et al. and Sharma et al. ${ }^{10,11}$ The responder rate ( $\geq 50 \%$ seizure reduction) of the observational study was less convincing than that reported in the recent Cochrane review. ${ }^{2}$ There may be numerous reasons for the difference in outcome as discussed in chapter 2 . Independent of the less convincing results on seizure reduction, there was a reduction in seizure clusters and a reduction in seizure severity in a substantial proportion of the patients. Previous literature about efficacy of the KD does not specify the effect on seizure clusters. Seizure severity is not often used as outcome measure of the KD. Hallböök at al described a statistically significant decrease in seizure severity after 3 months KD compared with baseline measured as the mean of the NHS3 value. ${ }^{12}$

Reduction in seizure clusters and seizure severity are, in our opinion, two valuable additional outcome parameters which can help to objectify seizure-related effects of the treatment, positive effects which can be the reason for patients and/or parents to continue treatment with the KD.

\section{Tolerability}

The most invalidating side-effects in the observational study, especially in the first three months of treatment, were gastro-intestinal symptoms which can usually be 
reduced by fine-tuning the diet. Growth deceleration was present in $30 \%$ of patients and weight reduction in $15 \%$. No patient developed ECG abnormalities or kidney stones and increase in lipid profile was rare. Improved arousal was mentioned in $30 \%$. In the RCT, an adapted version of the SIDAED questionnaire was used. Total scores of the nine different domains of side-effects showed no statistically significant differences between the KD and CAU-groups at either 6 weeks or at 4 months, but patients treated with the KD had a significantly higher score for gastrointestinal symptoms at these two time points. The mean value of gastro-intestinal symptoms in the KD-group increased after 6 weeks' treatment but declined after 4 months of treatment to a value that almost approached the baseline level. We concluded that fine-tuning the diet can reduce patients' symptoms. No patient developed ECG abnormalities or kidney stones. One child treated with the KD for four months showed a clinically relevant decrease in height, and in another child, there was relevant weight reduction. In the lipid profile, only the mean value for total cholesterol at 6 weeks' treatment with the KD was significantly higher compared to the value of the children in the CAU-group.

In both studies, gastro-intestinal problems were the most prominent side-effects. Although fine-tuning of the KD can reduce these symptoms in an essential number of patients, these side-effects need special attention, especially in the first months of treatment. Intensive supervision by the dietician and pediatrician are crucial because gastro-intestinal side-effects are frequently the cause of early discontinuation of the diet. Also, growth deceleration is a frequent side-effect in our studies as well as in the literature but it was never a reason to stop the treatment. Although described in the literature, none of the patients in our studies developed ECG abnormalities or kidney stones. Clinical relevant increase in lipid profile was rare and in most cases mild and correctable with dietary adjustments.

Based on the results of our study and recent literature, we reduced the frequency of the ECGs and blood examinations in daily clinical practice. In our opinion it is important to examine urine at each visit to the outpatient clinic because preventive precautions are indicated in case of urinary debris or an increased calcium/creatinine ratio to avoid the development of kidney stones.

\section{Retention}

After one and two years, one in three (16 patients (33\%)) and one in four (11 patients $(23 \%))$, respectively, were still on the diet. Eleven patients $(23 \%)$ discontinued the diet within the first three months after initiation, four of them before three weeks of treatment. Reasons for discontinuation before three months were side-effects, problems with compliance and increased seizure frequency or severity. Discontinuation after three months of treatment was mainly due to lack of efficacy, although problems with compliance and side-effects were still an issue. After one year 
of treatment, most patients continued the diet for a longer time. The mean time on the KD during the 2-year study period was 10.25 months (range 0.5-24 months).

In the RCT, two children stopped the KD before the first visit at six weeks because of side-effects. Three patients stopped treatment before the endpoint of four months. Two patients, in spite of being responders ( $\geq 50 \%$ seizure reduction), discontinued the KD because of gastro-intestinal side-effects, and another child because of a change in seizure pattern in which seizure occurrence shifted from sleep to daytime.

The high drop-out ratio before three months' treatment is in line with the literature. ${ }^{3}$ In the observational study, the retention rate at 12 months is somewhat lower compared with the study by Neal (54\%). The retention rates at one year are low compared to treatment with AEDs. In three studies in children with refractory epilepsy retention rates at 12 months were $55 \%$ for gabapentin ${ }^{13}, 49 \%$ for levetiracetam ${ }^{14}$ and $51 \%$ for clobazam, $37 \%$ for topiramate and $69 \%$ for lamotrigine. ${ }^{15}$ In case of no efficacy after 4 to 6 months' treatment with the KD the advice is to taper it off. ${ }^{16}$ The impact of a dietary treatment on the daily life of the patient and his family is greater than with medication. In case of no beneficial effect, the patient is not motivated to continue a dietary treatment.

\section{Psychological assessment}

One of the common areas that represent tolerability problems, is the change in cognition, behavior, mood and alertness. ${ }^{17}$

Our observational study was the first to use a comprehensive battery of cognitive tests, to be completed by the children themselves, combined with parent report questionnaires to assess the possible adverse effect of the KD on cognition, behavior and psychosocial adjustment. Fifteen school-aged children and adolescents were examined before the start of the KD and eleven were re-evaluated after approximately six months on the KD. Cognition showed a slight trend towards improvement in most patients. Psychosocial adjustment on the other hand, showed a small, not clinically relevant trend towards worsening. There was a tendency towards an increase in mood problems in two areas, namely fatigue/inertia and anger/hostility.

We concluded that there was no indication that the KD has a negative impact on cognition or social adaptation in the short term. There was a tendency towards an increase in mood problems which require adequate management in clinical practice.

We concluded finally that the KD is an effective therapy in children and adolescents in terms of reduction of seizure frequency and seizure clustering as well as in reduction of seizure severity, and that long-term use is well tolerated. After six months of treatment, it is obvious which patients are experiencing significant seizure reduction 
and tolerating the treatment well. Most of these patients will preserve the beneficial effects of the KD for a longer time.

\section{Ketogenic diet in adult patients}

At the beginning of our study, little was known about the ketogenic diet in adults with refractory epilepsy. Two articles about 4:1 classical KD were published in which slightly more than half of the adult patients were successfully treated. One of these articles dated back to 1930. Results of 4 adults treated with the MCT-KD were included in a report on children treated with the KD. In this article, treatment was classified as unsuccessful. ${ }^{18}$

We investigated whether the KD, especially the MCT-KD, was a good treatment option for adults with refractory epilepsy. We observed a decrease in seizure frequency, but to a lesser extent compared to our results in children. Five patients (33\%), two of whom proved to be responders, continued treatment for at least one year. $26.6 \%$ of all patients experienced at least one month of significant seizure reduction $(\geq 50 \%)$. Using an ITT analysis at twelve months, $13 \%$ of the patients were found to be responders. Most striking findings were firstly an improvement in mood and secondly an improvement in tension and anxiety. Quality of life seemed to be improving (mean value of QOL was increased during treatment). Nine patients reported an improved arousal during treatment. Improvement in mood and QOL was not related to seizure reduction (not associated with changes in seizure frequency). Although gastrointestinal complaints, weight loss and fatigue were reported frequently, only one patient discontinued the diet because of adverse effects. Mean ketone values were low. We must bear in mind that non-compliance may be higher in adults than in children.

Recently, one review and a meta-analysis have been published. ${ }^{19,20}$ Since the resurgence of the KD in the 1970s, there have only been 6 reports on KD treatment in adults with epilepsy (classical KD in 5, MCT in 1). Since the introduction of the MAD in 2006, five studies have evaluated MAD in adults with refractory epilepsy.

Klein et al. concluded that across all studies, 32\% of KD-treated and 29\% of MADtreated patients achieved $\geq 50 \%$ seizure reduction (Class III evidence). Most patients eventually stop the diet because of culinary and social restrictions. The anticonvulsant effect occurs quickly with both diets, within days to weeks. ${ }^{19}$ Potential barriers to large-scale use of the diets in adults include low rate of acceptance of the diet and high rates of diet discontinuation. Refusal to participate (in a trial) is commonly due to restrictiveness and complexity of the diet. In both KD and MAD studies, retention was poor. $^{19}$

Ye et al. performed a meta-analysis involving 12 studies (our study included) containing data from 270 patients. They concluded that a KD is a promising complementary therapy in adult intractable epilepsy, and while a classical ketogenic diet may be more effective, adult patients are likely to be less compliant with it than 
with a MAD. Although there are no direct comparisons of these two KD subtypes in adults, they advise starting with the MAD with a switch to the classical KD if greater seizure control is required. ${ }^{20}$

We concluded that the $K D$ is effective in adults with refractory epilepsy in reducing seizure frequency and well-tolerated although compliance was a concern. There was an improvement in mood and QOI scores independent of reduction in seizure frequency suggesting that the effects of the KD reach further than seizure control.

Based on the results of our study and recent literature, we changed daily clinical practice. Nowadays we offer adults with refractory epilepsy, who are eligible for a ketogenic diet therapy, the opportunity to start treatment with the MAD. For tube-fed adults, the classical KD remains the first choice.

\section{Is it useful to perform electro-encephalography (EEG) and specific laboratory investigations during treatment with the KD?}

\section{Can the interictal epileptiform discharge (IED) frequency be used as an early marker for the outcome of the KD?}

The reason for performing the EEG study was the desire to be able to predict the future therapeutic effect in individual patients at an early stage. The only relationship between IED changes and response to the KD was the decrease in nocturnal IEDs in responders to the KD. At individual level, however, the IED changes could not reliably predict which patients should be encouraged to continue the diet and so it is not informative for clinical practice.

Based on our results, it is not advised to use the EEG to predict the response to the KD.

On the subject of EEG changes after initiation of the KD, a study was published soon after ours which supports our conclusion. It was performed in a retrospective heterogeneous patient group $(n=43)$ with short routine EEGs in wakefulness at undefined periods before the KD initiation and repeated after undefined follow-up. Responders did not differ from non-responders regarding IED changes. ${ }^{21}$

\section{Does blood beta-hydroxybutyrate correlate better with seizure reduction due to the KD than ketones in the urine?}

The seminal feature of the KD is the production of ketone bodies ( $\beta$-hydroxybutyrate $(\mathrm{BHB})$, acetoacetate (AcAc) and acetone) by the liver. $^{22}$ 
In clinical practice, ketosis is measured in two different ways. BHB is measured in blood obtained by finger-prick and the level of ketones (mainly AcAc and a small amount of acetone) can be assessed in the urine using a dipstick. At the beginning of our studies it was common to measure ketosis in the urine to monitor the KD, probably because this was less invasive. BHB in blood seemed to correlate better with the amount of ketones in the brain. ${ }^{23}$ We wanted to know if blood betahydroxybutyrate correlates better with seizure reduction due to the KD than ketones in the urine.

In thirty-three patients (nineteen $<18$ years), the level of ketones was simultaneously measured in the urine and in blood at the three-monthly visits to the hospital. If the patient was able to collect urine, the urinary ketones were also measured at home every day. BHB measured after three and six months' treatment with the KD correlated with seizure reduction in a statistically significant way. The urinary ketones measured at the same time and the averaged values of the daily measured ketones in the urine did not correlate. At 9 and 12 months, we did not find any significance for either test but numbers were small.

In the cohort consisting of forty-eight children and adolescents, there was a significant correlation between being a responder and the single value of BHB in the capillary blood measured at the 12-, 15- and 18-month visits. No significant correlation was found for the mean of BHB or the mean of the urinary ketosis measured at home, nor for the single value of ketones in urine measured at each out-patient visit throughout the treatment.

In the interim analysis of the randomized controlled trial, the so-called KOEK-study involving twenty-six children and adolescents in the KD-group, there was a statistically significant correlation between being a responder at 6 weeks and the mean value of BHB measured in blood during the first six weeks of treatment. No other correlations were found at six weeks and no correlations at all were found at four months' treatment.

The relationship between seizure control and ketosis is, however, still unclear. ${ }^{3}$ Schoeler et al. conducted a literature review to identify factors that may influence response to ketogenic diet therapies (KDT). Ketosis was classified as providing 'weak evidence' of an effect on response to KDT, meaning that such an effect was reported in less than half of the cases investigated.

No correlation was found between seizure control and blood BHB in eleven publications concerning children, or in nine on adolescents or in four on adults. A correlation between improved seizure control and higher blood BHB was described in four publications on children, in two on adolescents and in one on adults. No correlation was found between seizure control and urinary ketosis in children in eight publications, in adolescents in seven publications and in adults in five publications. 
A positive correlation has been found between seizure control and urinary ketone levels in children in five publications, in adolescents in four publications and in adults in one publication. ${ }^{24}$

We concluded that it is better to measure $B H B$, even at a low frequency, than to measure urinary ketosis to monitor the KD. Ketosis measured in urine only indicates compliance and it is not a value to aim for in terms of efficacy. The value of BHB with regard to efficacy remains unclear.

\section{Are changes in serum levels of the neuropeptides cholecystokinin-8 (CCK-8) and leptin, during treatment with the KD, relevant?}

The mechanism underlying the KD's actions have remained elusive. The KD probably works through multiple mechanisms that target fundamental biochemical pathways linked to cellular substrates (e.g., ion channels) and mediators responsible for neuronal hyperexcitability. ${ }^{22}$ Currently, the focus is on changes in energy metabolism. ${ }^{25,26} \mathrm{~A}$ variety of (neuro-)peptides may be altered which could have a significant influence on neuronal excitability. ${ }^{26,27}$ Based on experimental animal studies, we were interested in the changes in serum levels of the neuropeptides, cholecystokinin-8 (CCK-8) and leptin. As far as we are aware, this is the first study on blood CCK-8 levels in humans on the KD. CCK-8 increased and leptin decreased during treatment with the KD. The increase of CCK- 8 was significant in responders after three and twelve months' treatment. Leptin values were decreased at three and twelve months. The decrease at three months was significant in men and post-pubertal women. It is plausible that the increase in CCK- 8 and the decrease in leptin contribute to the anti-convulsive effect of the KD.

It is too early to draw definitive conclusions about the role of CCK-8 and leptin in the mechanism of action of the KD. In our opinion, further research would be worthwhile. One of the pivotal questions is whether the rate of increase of CCK-8 may be an early predictor of effectiveness. We underline the importance of further unraveling the mechanism of action of the KD as a better understanding might be helpful in improving patient selection or in constructing a more easily applicable form of the KD.

\section{Is the ketogenic diet a cost-effective treatment for children with refractory epilepsy?}

Since resources are scarce, in current health care decision-making, we wanted to prove that the effects of the KD are worth the extra cost, to enable decisions to be evidence-based. To our knowledge, no model-based or trial-based study had been carried out on the cost-effectiveness of the KD. We performed an economic 
evaluation from a societal perspective which consisted of a cost-utility analysis with quality-adjusted life-year (QALY) as primary outcome and a cost-effectiveness analysis with treatment responder ( $\geq 50 \%$ seizure reduction) as the primary outcome. Since the study is still ongoing we report the interim analysis of the cost-effectiveness of the comparative period of the trial, i.e. the 4-month study period.

The KD is more expensive than care as usual (CAU) (resp. €20,986 and €15,245) and (almost) equally effective in terms of QALYs (resp. 0.253 and 0.250). The Incremental Cost-Effectiveness Ratio (ICER) represents the cost of an additional QALY gained and was used to estimate the cost-utility of KD as opposed to CAU. The cost per QALY rises high above any acceptable ceiling ratio which lies between $€ 20,000$ and $€ 80,000$ per QALY in The Netherlands, depending on the burden of disease. ${ }^{28}$ The ceiling ratio represents society's willingness to pay for the increase of one QALY. The incremental costs (KD: $€ 20,986$ - CAU: $€ 15,245$ ) divided by the incremental number of responders, taking the difference in group sizes into account (KD: 13/26 - CAU: 4/22), resulted in an ICER of $€ 18,044$ per responder. From a health care perspective, the mean costs per patient in the KD and CAU group are resp. $€ 12,139$ and $€ 7,589$, resulting in an ICER of $€ 14,300$ per responder.

In conclusion, this 4-month analysis shows that the KD is not cost-effective when including the QALY as a primary outcome parameter. The time horizon of the interim analysis is short which can be a reason for our inability to measure changes in QALYS or costs. The significant differences in costs were fully related to the intervention and diet costs in the KD group. Furthermore, the question arises whether the QOL instruments used in our study were sensitive enough to measure clinical changes (e.g. being a responder). In future studies, perhaps other preference-based QOL instruments would be more suitable and/or sensitive in children with epilepsy. For example, the Child Health Utility-9D (CHU-9D) ${ }^{29}$ or Assessment of Quality Of Life-6D $(A Q o L-6 D)^{30}$, both of which became available after the start of our trial, might be a welcome addition in studying QOL in children with epilepsy.

In 2014, our research group constructed a Markov decision analytical model with a 5 -year time horizon from a health care perspective. The objective was to estimate the expected cost-utility and cost-effectiveness of the KD, vagus nerve stimulation (VNS) and CAU. Data were derived from literature and expert meetings. After 12 months, the KD is cost-effective compared to VNS but compared to CAU, neither KD nor VNS are cost-effective options. After 5 years and at a ceiling ratio of $€ 80.000$, VNS had the highest probability (51\%) of being cost-effective followed by CAU (36\%) and KD (14\%). On average the benefits of KD and VNS fail to outweigh the costs of the therapies. ${ }^{31}$

Our results suggest that the benefits of KD fail to outweigh the costs of the therapy but the available information is still limited and the studies conducted have significant limitations. It is too early to draw definite conclusions. There is great need for randomized controlled trials with large patient samples, long-term follow-up periods 
and outcome measures that assess effects beyond seizure frequency (e.g. quality of life).

\section{What is the position of the KD as compared to other treatments for patients with refractory epilepsy?}

The literature contains a considerable amount of evidence that ketogenic diet therapies are the treatment of first choice for glut-1 deficiency syndrome and pyruvate dehydrogenase complex deficiency (PDHD) and there is growing evidence of the KD's efficacy in specific epilepsy syndromes, e.g. West syndrome, Dravet syndrome, Doose syndrome, Lennox-Gastaut syndrome, tuberous sclerosis complex etc. $^{16}$

Despite all the research, it is not yet possible to predict good responders, except for the above-mentioned disorders of brain metabolism. ${ }^{24}$ Recommendations of the International KD Study group are that the KD should be strongly considered in a child, regardless of age or gender, in whom two to three anticonvulsant therapies have failed, and particularly in those with symptomatic generalized epilepsies. In specific epilepsy syndromes - Dravet syndrome, infantile spasms, myoclonic-astatic epilepsy, tuberous sclerosis complex - the KD could be offered earlier. The KD is probably only of limited benefit in children who are candidates for epilepsy surgery.

In a recent publication, Felton summarized that the KD is a broad-spectrum treatment that can be used safely and effectively for most types of epilepsy and in patients of all ages from infancy through (late) adulthood, keeping the absolute contraindications in mind. ${ }^{32}$ In patients, not eligible for epilepsy surgery, in whom the next treatment step can be VNS or the KD, the KD is, in our opinion, the preferred next step. Efficacy of the KD will be clear in a shorter time, probably with lower costs. ${ }^{31}$

The possibilities offered by KD therapies continue to expand, making it feasible to choose the type of KD that best suits the patient and the family. Nowadays four major types of the KD exist, with an option of mixed forms (so-called "tailor made"). The statement that the KD is too restrictive and unpalatable is losing momentum due to new developments (e.g. new (liquid) formulae, new recipes, new methods of preparation). Nevertheless, treatment with a diet requires a change in lifestyle for the patient and families. Support of peers at school or work are valuable.

Advantage can be gained by giving information about the KD early on in the epilepsy treatment and especially as soon as medical therapy resistance becomes obvious. The nurse practitioner has a major role in informing patients and parents about the practical issues concerning the KD before referral to the complete multidisciplinary KD team where the dietician can provide more detailed information.

A multidisciplinary KD team consists of (child)neurologists, pediatricians, dietitians and nurses. ${ }^{33}$ As a consequence of the results of our trial in school-aged children, which show a tendency towards an increase in mood problems, a psychologist is now 
also part of our KD team. We have good experience with psychological counseling prior to and during the start of the KD in children. Advice is requested when there is doubt about the feasibility of the KD in a child or adult, especially with comorbid mental retardation, behavioral problems or psychiatric comorbidity.

The first weeks or even months of the treatment are crucial. Patients and their families are learning about the everyday implementation of the diet. In the case of side-effects, the dietician will fine-tune the diet. As soon as efficacy is evident, one knows that the efforts are worthwhile. In an essential number of patients, positive effects start to appear after two to three weeks of treatment with the KD, although later responses are common. Recommendations are to continue the KD for at least a mean of 3.5 months (SD 2.2 months) before tapering off because of ineffectiveness. In case of efficacy, the KD will be continued for a longer time. In children with $>50 \%$ seizure response, the KD is often discontinued after approximately 2 years. Children with glut-1 and pyruvate dehydrogenase deficiency may require longer KD durations than other conditions. ${ }^{16}$

\section{Which type of KD is preferable for which patient?}

In our opinion, an experienced KD center should be able to offer all types of KD therapies. Nevertheless, the preferences are often historically based and depend on resources. MCT emulsions are, for instance, not refunded in many countries. The specific patient's food preferences, the cooking creativity of the patient or parents, medical aspects like parenteral feeding or a social aspect such as living in an institution, all contribute to the final choice of which type of KD to start with. Our center started in the nineties with the MCT KD. Because of the less convincing results of our KD study in adults and the growing evidence in the literature, we nowadays offer adults the possibility of starting with the MAD. In infants and patients with a percutaneous gastrostomy tube, the liquid form of the classical KD will be used. This may be supplemented by oral feeding, if possible. In children, the MCT KD is most frequently advised since no complicated calculation programs are needed. In case of fine-tuning because of side-effects, an amount of MCT fat will be replaced by LCT fat. Depending on the specific situation, the decision can be made to use the MAD in adolescents or even younger children. Until now, we have only used the LGID if a patient explicitly asks for this form of KD.

Because of the above-mentioned reasons, treatment with the KD requires intensive involvement of the KD team prior to and during the first months of treatment. As shown in the cost-effectiveness analyses, the intervention and the intense supervision of the KD team, especially in the first months, makes the KD an expensive treatment. But the current lack of evidence concerning cost-effectiveness, does not mean that the $K D$ is not a valuable addition to the treatment arsenal. 


\section{Finally some recommendations and suggestions for future research}

The KD is a well-established treatment for patients - from infancy to early adulthood with refractory, non-surgical epilepsy. Successful therapy with KD requires an experienced multidisciplinary team in an academic or epilepsy center and a motivated patient embedded in a motivated family.

KD must be relieved from the stigma that it is the treatment of least choice. When two or three AED are found not to be able to control the epileptic seizures and epilepsy surgery is not possible, KD can be offered as an alternative treatment. It is the treatment of first choice for patients with glut-1 deficiency syndrome and PDHD. There is a need for RCTs with large patient samples, long-term follow-up and outcome measures that assess effects beyond seizure frequency. There is a need for trial-based studies on the cost-effectiveness of the KD. Future research should focus on identifying patients who benefit the most from the KD. Trials in homogenous patients groups, i.e. with the same epilepsy syndrome, in the same age group and with the same type of KD are preferred; meaning there is a need for multi-center or even international trials. Further research into the mechanism of action of the KD is important, as a better understanding of its action might help to improve patient selection or to construct a more easily applicable form of the KD. 


\section{References}

1. Levy R, Cooper P. Ketogenic diet for epilepsy. Cochrane Database Syst Rev 2003:CD001903.

2. Levy RG, Cooper PN, Giri P. Ketogenic diet and other dietary treatments for epilepsy. Cochrane Database Syst Rev 2012;3:CD001903.

3. Neal EG, Chaffe $\mathrm{H}$, Schwartz $\mathrm{RH}$, et al. A randomized trial of classical and medium-chain triglyceride ketogenic diets in the treatment of childhood epilepsy. Epilepsia 2009;50:1109-1117.

4. Sills MA, Forsythe WI, Haidukewych D, MacDonald A, Robinson M. The medium chain triglyceride diet and intractable epilepsy. Arch Dis Child 1986;61:1168-1172.

5. Hemingway C, Freeman JM, Pillas DJ, Pyzik PL. The ketogenic diet: a 3- to 6-year follow-up of 150 children enrolled prospectively. Pediatrics 2001;108:898-905.

6. Kwiterovich PO, Jr., Vining EP, Pyzik P, Skolasky R, Jr., Freeman JM. Effect of a high-fat ketogenic diet on plasma levels of lipids, lipoproteins, and apolipoproteins in children. JAMA 2003;290:912-920.

7. Kim JT, Kang HC, Song JE, et al. Catch-up growth after long-term implementation and weaning from ketogenic diet in pediatric epileptic patients. Clin Nutr 2012.

8. Ben-Menachem E, Sander JW, Privitera M, Gilliam F. Measuring outcomes of treatment with antiepileptic drugs in clinical trials. Epilepsy Behav 2010;18:24-30.

9. Bootsma HP, Ricker L, Hekster YA, et al. The impact of side effects on long-term retention in three new antiepileptic drugs. Seizure 2009;18:327-331.

10. Neal EG, Chaffe H, Schwartz RH, et al. The ketogenic diet for the treatment of childhood epilepsy: a randomised controlled trial. Lancet Neurol 2008;7:500-506.

11. Sharma S, Sankhyan N, Gulati S, Agarwala A. Use of the modified Atkins diet for treatment of refractory childhood epilepsy: a randomized controlled trial. Epilepsia 2013;54:481-486.

12. Hallbook T, Lundgren J, Rosen I. Ketogenic diet improves sleep quality in children with therapyresistant epilepsy. Epilepsia 2007;48:59-65.

13. Mills JK, Ruslan NE, Lewis TG, et al. Retention rate of Gabapentin in children with intractable epilepsies at 1 year. Seizure 2012;21:28-31.

14. Peake D, Mordekar S, Gosalakkal J, et al. Retention rate of levetiracetam in children with intractable epilepsy at 1 year. Seizure 2007;16:185-189.

15. Mills JK, Lewis TG, Mughal K, Ali I, Ugur A, Whitehouse WP. Retention rate of clobazam, topiramate and lamotrigine in children with intractable epilepsies at 1 year. Seizure 2011;20:402-405.

16. Kossoff EH, Zupec-Kania BA, Amark PE, et al. Optimal clinical management of children receiving the ketogenic diet: recommendations of the International Ketogenic Diet Study Group. Epilepsia 2009;50:304-317.

17. Farasat S, Kossoff EH, Pillas DJ, Rubenstein JE, Vining EP, Freeman JM. The importance of parental expectations of cognitive improvement for their children with epilepsy prior to starting the ketogenic diet. Epilepsy Behav 2006;8:406-410.

18. Schwartz RH, Eaton J, Bower BD, Aynsley-Green A. Ketogenic diets in the treatment of epilepsy: shortterm clinical effects. Dev Med Child Neurol 1989;31:145-151.

19. Klein P, Tyrlikova I, Mathews GC. Dietary treatment in adults with refractory epilepsy: A review. Neurology 2014;83:1978-1985.

20. Ye F, Li XJ, Jiang WL, Sun HB, Liu J. Efficacy of and patient compliance with a ketogenic diet in adults with intractable epilepsy: a meta-analysis. J Clin Neurol 2015;11:26-31.

21. Walker I, Said RR. Predictors of Ketogenic Diet Efficacy in Children Based on the Electroencephalogram (EEG). J Child Neurol 2014.

22. Rho JM. How does the ketogenic diet induce anti-seizure effects? Neurosci Lett 2015.

23. Morris AA. Cerebral ketone body metabolism. J Inherit Metab Dis 2005;28:109-121.

24. Schoeler NE, Cross JH, Sander JW, Sisodiya SM. Can we predict a favourable response to Ketogenic Diet Therapies for drug-resistant epilepsy? Epilepsy Res 2013;106:1-16.

25. Schwartzkroin PA. Mechanisms underlying the anti-epileptic efficacy of the ketogenic diet. Epilepsy Res 1999;37:171-180.

26. Giordano C, Marchio M, Timofeeva E, Biagini G. Neuroactive peptides as putative mediators of antiepileptic ketogenic diets. Front Neurol 2014;5:63. 
27. Rho JM, Stafstrom CE. The ketogenic diet: what has science taught us? Epilepsy Res 2012;100: 210-217.

28. Council for Public Health and Healt Care. Sensible and sustainable care (in Dutch). Zoetermeer: Council for Public Health and Health Care, 2006.

29. Stevens K, Ratcliffe J. Measuring and valuing health benefits for economic evaluation in adolescence: an assessment of the practicality and validity of the child health utility $9 \mathrm{D}$ in the Australian adolescent population. Value Health 2012;15:1092-1099.

30. Richardson JR, Peacock SJ, Hawthorne G, lezzi A, Elsworth G, Day NA. Construction of the descriptive system for the Assessment of Quality of Life AQoL-6D utility instrument. Health Qual Life Outcomes 2012;10:38.

31. de Kinderen RJ, Postulart D, Aldenkamp AP, et al. Cost-effectiveness of the ketogenic diet and vagus nerve stimulation for the treatment of children with intractable epilepsy. Epilepsy Res 2015;110: 119-131.

32. Felton EA, Cervenka MC. Dietary therapy is the best option for refractory nonsurgical epilepsy. Epilepsia 2015;56:1325-1329.

33. Lambrechts D, Gerrits-Troost C, Boomsma M. Voeding bij epilepsie. In: Informatorium voor Voeding en Diëtitiek- dieetleer: Bohn Stafleu Van Loghum, Houten, 2005: 1601-1627. 



\section{Chapter 12}

Summary

Samenvatting

Valorisatie

Dankwoord

List of publications

Curriculum Vitae 
Summary 


\section{Summary}

Chapter 1, the introduction, provides background information on refractory epilepsy and ketogenic diet therapies.

Chapter 2 presents the results of a long-term (i.e. twenty-four months), observational study on the ketogenic diet in forty-eight children with refractory epilepsy. Thirtyeight were treated with the MCT-diet. After one and two years, one in three and one in four, respectively, were still on the diet. Highest responder rate, in terms of more than $50 \%$ seizure reduction (i.e. $22.9 \%$ ), was seen at six and nine months of treatment. At other moments it was $16.7 \%$. Of the fifteen patients with seizure clusters during baseline, $60 \%$ responded with cluster reduction after three months and most of them were non-responders with respect to the total seizure frequency. From three months' treatment onwards, most of the patients showed a relevant decrease in seizure severity which was mainly related to the most severe seizure type. The most invalidating side-effects, especially in the first three months of treatment, were gastro-intestinal symptoms which can usually be reduced by fine-tuning the diet. Growth deceleration was present in $30 \%$ of patients and weight reduction in $15 \%$. No patient developed ECG abnormalities or kidney stones and increase in lipid profile was rare. Improved arousal was mentioned in $30 \%$. We concluded that the KD is an effective therapy in terms of reduction of seizure frequency and seizure clustering as well as in reduction of seizure severity, and that long-term use is well tolerated. After six months of treatment, it is obvious which patients are experiencing significant seizure reduction and tolerating the treatment well.

Chapter 3 presents the results of fifteen school-aged children and adolescents on the KD who were examined before the start of the KD and eleven who were re-evaluated after approximately six months on the diet. Possible adverse effect of the KD on cognition, behavior, psychosocial adjustment and quality of life (QOL) were evaluated using psychological tests for the children and parent report questionnaires. Our study was the first to use a comprehensive battery of cognitive tests, to be completed by the children themselves to assess the effect of the KD. Five out of fifteen patients showed a seizure reduction of more than $50 \%$. Six other patients remained on the diet because of improved alertness. Cognition showed a slight trend towards improvement in most patients. Psychosocial adjustment on the other hand, showed a small, clinically not relevant trend towards worsening. There was a tendency towards an increase in mood problems in two areas, namely fatigue/inertia and anger/hostility. We concluded that there was no indication that the KD has a negative impact on cognition or social adaptation in the short term. There was a tendency towards an increase in mood problems which require adequate management in clinical practice. 
Chapter 4 describes the results of fifteen adult patients with refractory epilepsy treated with the ketogenic diet. Overall psychological functioning (cognition, mood and quality of life) was measured.

Thirteen patients started on the MCT diet; two patients on the classical KD.

Five patients, two of whom proved to be responders, continued treatment for at least one year. $26.6 \%$ of all patients experienced at least one month of significant seizure reduction $(\geq 50 \%)$.

Common side-effects were gastrointestinal disorders, loss of weight and fatigue.

Psychological tests showed no significant changes. Nevertheless, nine patients reported an improved arousal during treatment. Most striking findings were firstly an improvement in mood and secondly an improvement in tension and anxiety. Mean value of QOL was increased during treatment. Improvements in mood and QOL were not related to seizure reduction.

Mean beta-hydroxybutyrate (BHB) values were low. We must bear in mind that noncompliance may be higher in adults than in children.

Chapter 5 describes the design of the so-called KOEK-study (KOsten Effectiviteit Ketogeen diet). A randomized controlled trial (RCT) is used to study the costeffectiveness of the KD compared with care as usual (CAU) in children and adolescents with intractable epilepsy. A trial-based and model-based economic evaluation, looking at the cost-effectiveness and cost-utility from a societal perspective, is embedded in this RCT.

Chapter 6 reports on the efficacy and tolerability of the KOEK-study during the first four months of the trial. Forty-eight children were included in the analyses of this study (twenty-six in the KD group, twenty-two in the CAU group). Using an intentionto-treat analysis at four months, 50\% of the participants in the KD group had a seizure reduction $\geq 50 \%$ from baseline, compared with $18.2 \%$ of the participants in the CAU group. Mean seizure frequency at four months compared with baseline was, after removal of two outliers in the KD group, significantly lower in the KD group than in the CAU group. Twice as many patients in the KD group had a relevant decrease in seizure severity score. Patients treated with the KD had a significantly higher score for gastrointestinal symptoms.

Chapter 7 reports on the interim outcomes of the economic evaluation of the KOEKstudy. The mean costs per patient in the CAU group were $€ 15,245$ compared to $€ 20,986$ per patient in the KD group, resulting in an ICER (incremental costeffectiveness ratio) of $€ 18,044$ per responder. We did not find any benefits in terms of Quality Adjusted Life Years (QALYs) and therefore, the costs per QALY rise high above any acceptable ceiling ratio resulting in unfavorable cost-utility ratios. The ceiling ratio is the society's willingness to pay for the increase of one QALY. Based on the costutility analysis, the KD is not a cost-effective treatment option. 
Chapter 8 describes the results of two different ways of measuring ketosis and the relation with seizure reduction. In thirty-three children and adults, betahydroxybutyrate (BHB) was measured in capillary blood obtained by finger-prick. The level of ketones was measured in the urine using a urine dipstick during the threemonthly visits to the hospital. If the patient was able to collect urine, the urinary ketones were also measured at home every day. BHB measured after three and six months' treatment with the KD correlated with seizure reduction in a statistically significant way. The urinary ketones measured at the same time and the averaged values of the daily measured ketones in the urine did not correlate. We concluded that it is better to measure BHB, even at a low frequency, than to measure urinary ketosis.

Chapter 9 evaluates the usefulness of calculating interictal epileptiform discharge (IED) frequency in a prospectively examined heterogeneous group of thirty-four epilepsy patients (children and adults) treated with a KD. Twenty-four-hour EEG recordings pre- and post-treatment enabled us to examine the effect of the KD on the IED frequency in wakefulness as well as in sleep. The objective was to predict the future effect of the KD in individual patients at an early stage (at six weeks of treatment). Because the diet is a burden, early, useful information to help to decide whether to (dis)continue the diet, is welcome. Nocturnal decrease of IEDs at six weeks was related to being a responder to the KD. However, at individual level, the IED changes could not reliably predict which patients should be encouraged to continue the diet. Because a clear reduction in seizure frequency was obvious in the second month of KD treatment in all responders, it is better to base this decision on seizure counts.

Chapter 10 evaluates the changes in serum levels of the neuropeptides, cholecystokinin-8 (CCK-8) and leptin, in fifty-four children and adults with refractory epilepsy treated with the KD for at least three months. Twenty-six patients continued the KD for at least one year. After three and twelve months, respectively, 39\% and $26 \%$ were responders. CCK-8 values were significantly increased in responders after three and twelve months' treatment, but not in non-responders. Leptin values were decreased at three and twelve months. The decrease at three months was significant in men and post-pubertal women. This is the first study to evaluate the course of CCK8 and leptin in humans with refractory epilepsy treated with the KD. In our opinion it is plausible that the increase in CCK-8 and the decrease in leptin contribute to the anti-convulsive effect of the KD.

Chapter 11 presents a discussion of the overall findings and suggests future research options. 
Samenvatting 


\section{Samenvatting}

Hoofdstuk 1, de inleiding, geeft achtergrondinformatie over therapieresistente epilepsie en de behandeling daarvan met het ketogeen dieet (KD).

Hoofdstuk 2 gaat over de langetermijnresultaten (24 maanden) van 48 kinderen met therapieresistente epilepsie die behandeling met het KD startten. De meesten zijn behandeld met het MCT- dieet. Na één en twee jaar volgden respectievelijk nog één op drie en één op vier kinderen het KD.

$\mathrm{Na}$ zes en negen maanden behandeling werd het hoogste percentage (22.9\%) kinderen met meer dan de helft aanvalsvermindering bereikt. Op de andere meetmomenten was dit $16.7 \%$. Van de vijftien kinderen die voorafgaand aan het KD aanvalsclusters hadden, liet $60 \%$ een reductie van meer dan de helft van die clusters zien. De meesten van deze kinderen waren geen responders voor het totaal aantal aanvallen. Vanaf drie maanden behandeling bereikten de meeste kinderen een relevante daling van de aanvalsernst die veelal verbonden was met zijn/haar zwaarste type aanval.

Maag- en darmproblemen waren de meest invaliderende bijwerkingen. Deze kwamen voornamelijk tijdens de eerste drie maanden van behandeling voor. Meestal kon aanpassing van het dieet deze bijwerkingen verminderen. Bij 30\% van de kinderen vertraagde de lengtegroei en bij 15\% nam het gewicht af. Er deden zich geen ECGafwijkingen of nierstenen voor. Er trad zelden een noemenswaardige afwijking in het lipidenprofiel op. $30 \%$ van de kinderen was fitter.

We concluderen dat het KD een effectieve behandelmethode is die kan leiden tot afname van het totaal aantal aanvallen, van het aantal aanvalsclusters en van de aanvalsernst. Het KD wordt goed verdragen bij langdurig gebruik. Na zes maanden is duidelijk bij wie het dieet helpt en wie het ook goed verdraagt.

Hoofdstuk 3 beschrijft de resultaten van toepassing van het KD bij vijftien schoolgaande kinderen en adolescenten. Ze zijn allemaal voorafgaand aan de start van het KD onderzocht. Elf kinderen zijn opnieuw onderzocht nadat ze ongeveer zes maanden het KD volgden. Mogelijke bijwerkingen van het KD op cognitie, gedrag, psychosociale aanpassing en kwaliteit van leven (QOL) zijn onderzocht door de kinderen psychologisch te onderzoeken en de ouders vragenlijsten te laten invullen. Onze studie is de eerste waarin de kinderen psychologisch werden onderzocht.

Bij vijf van de vijftien kinderen was er een aanvalsreductie van meer dan $50 \%$. Voor zes andere kinderen was toegenomen alertheid de reden om het dieet voort te zetten. We vonden geen statistisch significante effecten maar de cognitie toonde een lichte trend naar verbetering. Psychosociale aanpassing daarentegen toonde een lichte, klinisch niet relevante trend richting slechtere resultaten. Verder zagen we een tendens van toegenomen problemen met stemming in het domein van moeheid/ inertie en boosheid/vijandigheid. 
We concluderen dat er geen indicatie is dat het KD op korte termijn een negatieve invloed heeft op cognitie en sociale aanpassing. De waargenomen tendens van stemmingsproblemen vraagt om bijzondere aandacht in de klinische praktijk.

Hoofdstuk 4 beschrijft de resultaten van het KD bij vijftien volwassen patiënten met therapieresistente epilepsie. Ook hebben we hun psychisch functioneren (cognitie, stemming en kwaliteit van leven) in kaart gebracht. Dertien patiënten startten met het MCT-dieet en twee met de klassieke vorm.

Vijf personen, waarvan twee responders, volgden het dieet minstens een jaar. Bij $26,6 \%$ van alle patiënten was er minstens een maand met significante aanvalsreductie ( $\geq 50 \%$ ). Maag- en/of darmproblemen, gewichtsverlies en vermoeidheid bleken de meest voorkomende bijwerkingen.

Psychologisch onderzoek toonde geen significante veranderingen. Toch gaven negen patiënten aan dat ze zich tijdens het dieet fitter voelden. De meest opvallende bevindingen waren verder een afname van somberheid en van spanning en angst. De gemiddelde waarde op de kwaliteit-van-leven-vragenlijst nam toe tijdens behandeling. De verbeterde stemming en $\mathrm{QOL}$ waren niet gerelateerd aan een verminderde aanvalsfrequentie. De gemiddelde beta-hydroxybutyraat (BHB)-waardes waren laag. We dienen er rekening mee te houden dat het voor volwassenen moeilijker kan zijn om zich strikt aan het dieet te houden.

Hoofdstuk 5 beschrijft de opzet van de zogenoemde KOEK-studie (KOsten Effectiviteit Ketogeen dieet). In deze gerandomiseerde gecontroleerde studie is de kosteneffectiviteit van het KD vergeleken met de gebruikelijke zorg bij kinderen en adolescenten met therapieresistente epilepsie. $\mathrm{Er}$ is een model-based en een trialbased economische evaluatie uitgevoerd. Hierbij is vanuit een maatschappelijk perspectief naar de kosteneffectiviteit en kostenutiliteit gekeken.

Hoofdstuk 6 beschrijft de effectiviteit en bijwerkingen bij 48 kinderen (26 kinderen in de KD-groep) in de eerste vier maanden van de KOEK-studie. Bij een 'intention to treat analyse' liet de helft van de kinderen die werden behandeld met het KD een aanvalsafname van meer dan $50 \%$ zien, in vergelijking tot de periode voorafgaand aan de start van het onderzoek. Bij de kinderen die hun gebruikelijke behandeling continueerden (care as usual (CAU)), gebeurde dit bij $18,2 \%$. Na het uitsluiten van twee uitschieters in de KD-groep was de gemiddelde aanvalsfrequentie na vier maanden significant lager in de KD-groep dan in de CAU-groep. Ook hadden twee keer zoveel patiënten in de KD-groep een relevante afname van de score van de aanvalsernst. De kinderen in de KD-groep toonden een significant hogere score voor maag- en darmproblemen.

Hoofdstuk 7 rapporteert de interimgegevens van de economische evaluatie van de KOEK-studie. De gemiddelde kosten per patiënt in de CAU-groep bedragen $€ 15.245$ 
vergeleken met $€ 20.986$ per patiënt in de KD-groep. Dit resulteert in een Incrementele Kosten-EffectiviteitsRatio (IKER) van €18.044 per responder. We vonden geen toename van voor kwaliteit gecorrigeerde levensjaren (QALY's). Zodoende kwamen de kosten per QALY ver boven alle geaccepteerde ceiling ratio's uit. De ceiling ratio is het bedrag dat de maatschappij bereid is om te betalen voor één QALY. Uit het oogpunt van een kostenutiliteitanalyse, geldt het KD niet als een kosteneffectieve behandeling.

Hoofdstuk 8 beschrijft de resultaten van twee manieren om de mate van ketose te meten en de relatie hiervan met de aanvalsvermindering. Bij 33 kinderen en volwassenen is tijdens de driemaandelijkse controles beta-hydroxybutyraat (BHB) in capillair bloed - verkregen via een vingerprik - gemeten, evenals de ketose in urine door middel van dipstick. Als het mogelijk was om thuis urine op te vangen, werd dagelijks ketose in urine bepaald.

BHB gemeten na drie en na zes maanden behandeling met KD, correleerde op een statistisch significante manier met de aanvalsreductie. Dit was niet het geval voor de ketosewaarden gemeten in urine (op hetzelfde meetmoment als BHB) en ook niet voor de gemiddelde waarden van de dagelijkse ketosebepalingen die thuis doorgingen. We concluderen dat het beter is om laagfrequent BHB te bepalen in capillair bloed dan vaak ketosewaarden te bepalen in urine.

Hoofdstuk 9 evalueert het nut van het bepalen van de hoeveelheid interictale epileptiforme ontladingen (interictal epileptiform discharges = IEDs) in een prospectief onderzochte, heterogene groep van 34 epilepsiepatiënten (kinderen en volwassenen) behandeld met het KD. Met 24-uurs-EEG's voorafgaand aan en na de start van de behandeling, is onderzocht wat het effect van het KD is op de hoeveelheid IEDs in waak en in slaap. Doel is om het effect van het KD voor een individu op een vroeg moment in de behandeling (na zes weken) te voorspellen. Het is zwaar om het KD vol te houden. Daarom is het gewenst om vroeg in het behandeltraject over informatie te kunnen beschikken waarop kan worden gebaseerd of het continueren van het dieet kansrijk is.

Een afname van de nachtelijke hoeveelheid IEDs na zes weken behandeling met KD was gerelateerd aan het zijn van een responder. Maar op individueel niveau konden de veranderingen in IEDs niet betrouwbaar voorspellen of een patiënt al dan niet zou moeten worden aangemoedigd om het dieet te blijven volgen. Omdat een duidelijke reductie in aanvalsfrequentie in de tweede maand van het $\mathrm{KD}$ bij alle responders is gezien, kan het tellen van het aantal aanvallen hier beter voor benut worden.

Hoofdstuk 10 rapporteert de veranderingen in serumconcentratie van de neuropeptides cholecystokinine-8 (CCK-8) en leptine bij 45 kinderen en volwassenen met therapieresistente epilepsie die minstens drie maanden zijn behandeld met het KD. Dit is het eerste onderzoek waarin de evolutie van CCK-8- en leptinewaardes tijdens behandeling met KD bij personen met therapieresistente epilepsie is 
onderzocht. Het dieet is door 26 patiënten minstens een jaar gevolgd. Na drie maanden had $39 \%$ van hen en bij twaalf maanden $26 \%$ van hen meer dan $50 \%$ aanvalsreductie. CCK-8 waardes waren statistisch significant verhoogd na drie en na twaalf maanden behandeling. Deze stijging was enkel bij de responders significant. De leptinewaarde was zowel na drie, als na twaalf maanden gedaald. Deze daling was na drie maanden behandeling statistisch significant bij mannen en bij vrouwen na de puberteit. Het is daarom aannemelijk dat de stijging van CCK-8 en de daling van leptine bijdragen aan het anti-convulsieve effect van het KD.

Hoofdstuk 11 brengt de bevindingen van alle studies met elkaar in verband en adviseert over toekomstig onderzoek. 
Valorisatie 


\section{Valorisatie}

\section{Vertaling van wetenschap naar maatschappij}

De Vereniging van Universiteiten (VSNU) definieert valorisatie als "Het proces van waardecreatie uit kennis, door kennis geschikt en/of beschikbaar te maken voor economische en/of maatschappelijke benutting en geschikt te maken voor vertaling in concurrerende producten, diensten, processen en nieuwe bedrijvigheid", (VSNU Raamwerk valorisatie-indicatoren).

Met andere woorden nieuw ontwikkelde kennis en technologie wordt aangewend ten gunste van de maatschappij (geïnspireerd op www.valoriseren.nl).

In het valorisatiehoofdstuk beschrijven we de concrete resultaten van dit proefschrift en de manier waarop deze resultaten van waarde kunnen zijn voor de maatschappij. Tevens worden de doelgroepen (ook buiten het medische veld) beschreven voor welke deze nieuwe informatie van waarde zou kunnen zijn en op welke manier we kennis kunnen delen. Vervolgens worden de mogelijkheden om de resultaten om te zetten in concrete producten en innovatie toegelicht. Tot slot wordt besproken hoe we de verworven kennis kunnen vertalen in een vervolgtraject.

\section{Maatschappelijke relevantie van wetenschappelijk onderzoek over epilepsie}

De onderzoeksvragen die behandeld worden in dit proefschrift zijn geformuleerd tijdens mijn klinische werk binnen het Academisch Centrum voor Epileptologie Kempenhaeghe/Maastricht UMC+. Patiënten worden verwezen naar de hooggespecialiseerde zorg van een derdelijnscentrum wanneer de tweedelijnszorg niet meer toereikend is. Vaak is de reden voor verwijzing de vraag naar meer of betere behandelingsmogelijkheden van de tot dat moment onbehandelbaar gebleken epileptische aanvallen.

De prevalentie van epilepsie bedraagt wereldwijd ca. $0,8 \%$. Bij ongeveer $30 \%$ van deze mensen is de epilepsie therapieresistent. Meerdere studies tonen aan dat mensen met chronische epilepsie een lagere levenskwaliteit en levensverwachting hebben, een lager niveau op onderwijsgebied halen en minder goed kunnen deelnemen in het beroepsleven. Psychologische en cognitieve problemen komen vaker voor en er is een hoge consumptie van de gezondheidszorg. ${ }^{1}$

Verminderen van de frequentie en ernst van epileptische aanvallen zal voor de patiënt maar ook voor zijn/haar directe omgeving en voor de maatschappij voordelen opleveren.

Voor een derdelijnscentrum als Kempenhaeghe is het belangrijk om een voorloper te zijn op het gebied van diagnostiek en behandeling. Wetenschappelijk onderzoek en innovatie zijn hiervoor onontbeerlijk. 
Wetenschappelijk onderzoek waarbij de vragen direct vanuit de klinische praktijk worden gegenereerd om uiteindelijk de patiëntenzorg te verbeteren, is maatschappelijk relevant.

\section{In hoeverre zijn de onderzoeksvragen in dit proefschrift relevant voor de verbetering van de behandeling van epilepsie?}

\section{Resultaten}

Effectiviteit van het ketogeen dieet (KD) bij kinderen en adolescenten is aangetoond in een observationele studie waarbij de follow up van de patiënten twee jaar duurde en in een gerandomiseerde gecontroleerde studie. In de observationele studie werd naast afname van de aanvalsfrequentie met meer dan $50 \%$, bij een aantal van de kinderen ook een afname van de hoeveelheid aanvalsclusters en een afname van de ernst van de aanvallen gezien. Tevens werd een deel van de kinderen alerter. Er deden zich geen cardiale complicaties van de behandeling voor ofschoon dit in eerdere literatuur wel werd beschreven.

$\mathrm{Bij}$ de interimanalyse op vier maanden van de gerandomiseerde gecontroleerde studie is bij $50 \%$ van de kinderen en adolescenten een aanvalsvermindering van meer dan $50 \%$ vastgesteld, tegenover zo'n aanvalsvermindering bij slechts $18,2 \%$ van de kinderen die hun gebruikelijke behandeling met medicatie continueerden. Bij patiënten behandeld met het KD is bij dubbel zoveel kinderen een relevante afname van de aanvalsernst gezien.

Bij kinderen en adolescenten zijn geen nadelige effecten op cognitie en sociale aanpassing tijdens behandeling met het KD gezien. $\mathrm{Er}$ is wel een trend tot toename van stemmingsproblemen (boosheid en vijandigheid) gezien die klinische aandacht vergt.

Bij volwassenen behandeld met het $K D$ is eveneens, maar in mindere mate, een afname van de aanvalsfrequentie gezien. Bij volwassenen is ook een afname van angst, spanning en somberheid gezien. Kwaliteit van leven lijkt te verbeteren. Het blijkt voor volwassenen moeilijker om het dieet strikt te volgen.

Opvolgen van de mate van ketose door middel van een vingerprik om de waarde van beta-hydroxyboterzuur te bepalen correleerde significant met de mate van aanvalsreductie. Het is beter om BHB in bloed te bepalen dan ketose in urine te meten, ook als BHB laagfrequent bepaald wordt.

$\mathrm{Bij}$ de groep responders op het ketogeen dieet is er na zes weken behandeling een afname van de nachtelijke hoeveelheid interictale epileptiforme ontladingen (interictal epileptiform discharges = IEDs). Maar op een individueel niveau, konden de IED-veranderingen niet betrouwbaar voorspellen welke patiënten aangemoedigd moeten worden om het dieet te blijven volgen. 
Bij de interimanalyse van de economische evaluatie na vier maanden is geen toename van QALYs (voor kwaliteit gecorrigeerde levensjaren) gevonden en zodoende komt de kost per QALY ver boven alle geaccepteerde ceilingratio's uit. Dit leidt tot ongunstige kosten-utiliteit ratio's. Gebaseerd op een kostenutiliteit-analyse, is het KD op korte termijn dus geen kosten-effectieve behandeling.

$\mathrm{Bij}$ onderzoek van de veranderingen in serumconcentratie van de neuropeptides cholecystokinine-8 (CCK-8) en leptine is vastgesteld dat de leptinewaardes dalen tijdens behandeling en de CCK waardes stijgen. Stijging van de CCK-8 waardes is bij de responders statistisch significant.

\section{Relevantie onderzoeksresultaten en gevolgen voor de dagelijkse praktijk}

Door het aantonen van de effectiviteit van het KD in meerdere leeftijdsgroepen en ten aanzien van meerdere aspecten zoals aanvalsfrequentie, afname van aanvalsclusters en afname van de aanvalsernst krijgt het KD meer aandacht en zullen meer patiënten met therapieresistente epilepsie met het dieet behandeld worden. Tijdens de langdurige follow-up van patiënten met het dieet zijn geen cardiale complicaties van de behandeling gezien. Dit heeft ertoe geleid dat een hartfilmpje minder frequent wordt gemaakt. Sinds de trend tot toename van stemmingsproblemen (boosheid en vijandigheid) bij kinderen behandeld met KD is vastgesteld, is hier klinisch meer aandacht voor. Een psycholoog maakt nu deel uit van het behandelteam en begeleidt het kind indien nodig tijdens het behandeltraject. Voor volwassenen is het moeilijker om het MCT-KD of klassiek KD vol te houden. Deze vaststelling heeft ertoe geleid dat volwassenen inmiddels worden behandeld met het gemodificeerde Atkins dieet dat minder strikt is en volgens recente literatuur even effectief is. Interimanalyse van de economische evaluatie levert geen wetenschappelijk bewijs voor de kosteneffectiviteit van het ketogeen dieet. Mede gezien het een interim analyse betreft, heeft deze bevinding geen gevolgen voor de dagelijkse praktijk.

\section{Doelgroepen}

Aangezien dit proefschrift klinisch onderzoek betreft, is de belangrijkste doelgroeppatiënten met epilepsie (kinderen, adolescenten en volwassenen). De resultaten hebben een brede maatschappelijke relevantie.

De onderzoeksresultaten dragen bij aan de kennis van alle disciplines die deel uitmaken van een multidisciplinair KD- team: neurologen en kinderneurologen, kinderartsen, diëtisten, verpleegkundig specialisten en psychologen.

De gepubliceerde artikelen zijn beschikbaar voor de bedrijven die vetemulsies, vitamines en minerale supplementen voor het KD leveren.

De resultaten van een economische evaluatie zijn van belang voor de professionals zelf, voor beleidsmakers in ziekenhuizen, zorgverzekeraars en de overheid. 
Table 12.1 Doelgroepen met gerelateerde activiteiten en producten.

\begin{tabular}{ll}
\hline Doelgroep & Activiteit/product \\
\hline Patiënten & KD koekboeken \\
& KD website \\
& Workshops \\
& Publicatie media \\
& Poeder voor MCT-emulsie \\
& Presentaties \\
Professionals & Wetenschappelijke artikelen \\
& Uitleg door culinair specialisten \\
& Implementatie resultaten in richtlijnen \\
Zorgverzekeraars & Wetenschappelijke artikelen \\
Industrie & Wetenschappelijke artikelen \\
& Poeder voor MCT-emulsie \\
Wetenschappers & Wetenschappelijke artikelen \\
& Presentaties \\
\end{tabular}

\section{Kennisoverdracht}

Wetenschappelijke kennis wordt waardevoller als deze niet alleen gedeeld wordt met vakgenoten, maar ook toegankelijk is voor derden zodat nieuwe combinaties van kennis kunnen leiden tot innovatieve oplossingen. Hierbij is de overdracht van kennis nodig (uit www.valoriseren.nl).

De beschreven onderzoeksresultaten hebben geleid tot diverse wetenschappelijke publicaties in internationale en nationale medische vaktijdschriften. $\mathrm{Er}$ is eveneens gepubliceerd in diverse publieksmedia. De onderzoeksresultaten hebben geleid tot wetenschappelijke voordrachten.

Samen met de Landelijke Werkgroep Ketogeen Dieet is een zorgpad 'Ketogeen dieet behandeling bij refractaire epilepsie en metabole ziekten bij kinderen' geschreven', hierin zijn onderzoeksbevindingen van huidig onderzoek verwerkt. Dit document is beschikbaar voor alle professionals.

\section{Innovatie}

Om de compliance met het dieet te verbeteren hebben culinair specialisten nieuwe, smakelijke recepten ontwikkeld voor zowel het MCT-dieet, het klassieke ketogeen dieet en gemodificeerde Atkins dieet. Ten einde de MCT-emulsie gemakkelijker te kunnen nuttigen is een poeder ontwikkeld waarmee de emulsie kan worden omgezet naar een in gerechten te gebruiken crème, pasta of schuim. Op een speciale website (www.ketogeenmenu.nl) zijn deze recepten en informatie over het KD beschikbaar. Recent is een interactief gedeelte aan de website toegevoegd waarin de recepten volgens behoefte van elke specifieke gebruiker op maat kunnen worden berekend. Om patiënten en hun ouders vertrouwd te maken met de nieuwe manier van voedsel 
bereiden werden workshops gegeven. Ontwikkeling van de digitale toepassingen zijn mogelijk gemaakt door fonds NutsOhra, CZFonds, Epilepsiefonds en ZonMw.

\section{Toekomst}

Het KD is een effectieve behandeling met aanvaardbare potentiële bijwerkingen. Langdurig consequent uitvoeren van het dieet kan moeilijk zijn maar is wel van het grootste belang voor de effectiviteit. Het is belangrijk om het dieet te blijven verbeteren en de acceptatie door patiënten en ouders te vergroten. Vervolgonderzoek kan zich verder richten op effectiviteit en kosteneffectiviteit van nieuwere en minder restrictieve vormen van het ketogeen dieet zoals het gemodificeerde Atkins dieet of laag glykemisch index dieet. De genoemde website zal voortdurend worden uitgebreid met nieuwe gerechten en toepassingen die de ruimte tot zelfmanagement van patiënten en ouders vergroten.

Het is nog niet mogelijk om goed te voorspellen wie baat zal hebben bij gebruik van het ketogeen dieet. Het is van belang om verder te zoeken naar wegen om deze patiëntengroep zo goed mogelijk te kunnen identificeren. Ook dient onderzoek te worden verricht naar welke vorm van het KD voor welke patiënten het best uitvoerbaar is en de minste bijwerkingen geeft. Vervolgonderzoek naar indicatoren is eveneens noodzakelijk.

Kosteneffectiviteit van het KD-gebruik op korte termijn, is momenteel nog niet aangetoond. Resultaten van lange termijn follow-up (16 maanden) zijn binnen afzienbare tijd beschikbaar. Verder onderzoek in grotere patiëntengroepen en met andere vormen van het ketogeen dieet zijn nodig.

Het werkingsmechanisme van het KD dat complex en multifactorieel is bepaald, is nog niet opgehelderd en vraagt om verder onderzoek. Als het werkingsmechanisme duidelijk is, worden wellicht ook mogelijkheden zichtbaar om het dieet te vereenvoudigen of te vergemakkelijken.

Voor het wetenschappelijk onderzoek op het gebied van het KD, maar ook voor onderzoek naar alle andere behandelmogelijkheden voor patiënten met refractaire epilepsie, is het van belang om naast effectiviteit als de bekende primaire uitkomstmaat - dat wil zeggen: aanvalsfrequentiereductie van minstens $50 \%$ - ook andere uitkomstmaten uit te werken. Hierbij kan gedacht worden aan aanvalsernst, effect op frequentie van aanvalsclusters en status epilepticus en kwaliteit van leven van de patiënt zelf en het omringende gezin. 


\section{Referenties}

1. Laxer KD, Trinka E, Hirsch $L$, et al. The consequences of refractory epilepsy and its treatment. Epilepsy Behav 2014;37:59-70.

2. Van der Louw EJTM, Van den Hurk TAM, Catsman- Berrevoets CE, et al. Zorgpad ketogeen dieet behandeling bij refractaire epilepsie \& metabole ziekten bij kinderen. Behandelarenversie. Epilepsie Vereniging Nederland, VKS (Volwassenen, Kinderen en Stofwisselingsziekten), Samenwerkingsverband Ketogeen Dieet Nederland In, 2015. 
Dankwoord 


\section{Dankwoord}

Mijn eerste onderzoek over ketogeen dieet startte elf jaar geleden in Kempenhaeghe. Het was een lange weg met veel ups en downs. Veel hindernissen dienden overwonnen te worden. Het doel is nu bereikt, het proefschrift is af en ik kijk tevreden terug op de voorbije jaren.

Mijn dank is groot voor de hulp, steun en bemoedigende woorden die ik van velen heb gekregen.

Als eerste wil ik alle patiënten, hun ouders en verzorgenden bedanken voor deelname aan het onderzoek. Ik heb grote bewondering voor de enthousiaste manier waarop werd samengewerkt. Jullie hebben extra tijd en energie gespendeerd aan het invullen van gedetailleerde aanvalskalenders, vragenlijsten etc. en dit samen met het leren van een dieet dat zeker in het begin niet eenvoudig is. Zonder jullie hulp was er nu geen proefschrift.

Beste promotoren en copromotor, het is een eer om met een klavertje vier als begeleiders te mogen promoveren. Door ieders inbreng ben ik gegroeid tot een wetenschapper maar ook gegroeid als mens en dat is nog waardevoller. Dank voor jullie geduld en vertrouwen in het slagen van deze promotie.

Prof. dr. H.J.M. Majoie, beste Marian, jij bent de rode draad door het hele verhaal. Je altijd positieve en constructieve aanpak viel me al op toen ik je, zeventien jaar geleden tijdens m'n sollicitatiegesprek in Kempenhaeghe, leerde kennen. Jarenlang hebben we 'tweewekelijks' overleg gehad om alle ins en outs van het ketogeen dieet onderzoek en de innovatieve projecten die er hand in hand mee gingen te bespreken. Voor elk probleem en elke tegenslag werd een gepaste oplossing bedacht en uitgewerkt. Niet denken in problemen maar in oplossingen, heb ik van jou geleerd. Recent ben je tot hoogleraar benoemd. Ik ben er trots op de eerste promovendus te zijn die jou als promotor mag noemen. Dank voor alles.

Prof. dr. A.P. Aldenkamp, beste Bert, je stond mee aan de wieg van het ketogeen dieet onderzoek. Vele jaren geleden, tijdens het uitvoeren van de observationele studie ketogeen dieet zei je: “Nog een tandje bijsteken en je maakt van je onderzoek een promotie". Het tandje bleek toen nog te groot. Het lukte me niet om het te combineren met een gezinsleven met jonge kinderen en lange reisafstanden. Bedankt om 'het tandje' een tijd te bewaren en tegelijkertijd het ketogeen dieet onderzoek met onverminderd enthousiasme te blijven begeleiden. Met elke statistiekvraag kon ik bij je terecht. Telkens wanneer ik in details dreigde te verzanden kon je heel snel aangeven waar de essentie zich bevond. Dank ook voor je waardevolle begeleiding en goede adviezen bij de laatste fase van het promotietraject. 
Prof. dr. J.S.H. Vles, beste Hans, met de start van het kosteneffectiviteit onderzoek ketogeen dieet werd het écht serieus voor me. Dit keer maakte ik de bewuste keuze om er een promotietraject van te maken. Je hebt me goed geholpen bij het maken van deze keuze en bij het overzien van de te nemen stappen. Je deskundigheid als kinderneuroloog en wetenschapper waardeer ik ten zeerste. Als geen ander kan je in een snel tempo analyseren en krachtig to the point formuleren. Je reacties op de artikelen en teksten zag ik met plezier tegemoet. Inhoudelijk sterk, alvast een voorbereiding op de latere verdediging en een complimentje of wijze woorden als toemaatje erbij. Hartelijk dank hiervoor.

Dr. A.J.A. de Louw, beste Anton, je initiatief als medisch hoofd om voor de kandidaatpromovendi onder de neurologen aan aantal maanden 'schrijftijd' te creëren in 2014 was dé bepalende factor. Het werd mogelijk om in een korte tijdspanne grote stappen te zetten. Het tempo van publicaties nam toe en het plezier in het onderzoekswerk groeide snel. Dank voor je enthousiaste en humoristisch opmerkingen. Het deed deugd te horen dat ik "goed op schema liep" telkens wanneer me weer de angst bekroop achter te lopen op schema.

Graag wil ik de leden van de beoordelingscommissie dr. C.E. Catsman-Berrevoets, prof. dr. L. Lagae, prof. dr. R. Ponds, prof. dr. L.J.I. Zimmerman, onder voorzitterschap van prof. dr. R.J. van Oostenbrugge van harte danken voor het lezen en beoordelen van mijn proefschrift.

Er zijn zoveel mensen die direct of indirect aan de totstandkoming van mijn proefschrift hebben bijgedragen. Allemaal experts in hun vak. Vaak zeer gedreven mensen, deskundig en bekwaam, enthousiast en gemotiveerd, steeds bereid om te helpen. Dank allemaal.

Een aantal teams en personen wil ik graag met naam noemen.

Dank aan mijn collega's, alle neurologen en kinderneurologen van Kempenhaeghe. Dank voor de oprechte belangstelling, de steun en goede adviezen die ik door de vele jaren heen van jullie heb gekregen. Een bijzonder woord van dank wil ik richten tot Richard Lazeron en Jurgen Schelhaas voor het overnemen van een deel van mijn poliklinische en klinische werk. Hierdoor heb ik de schrijftijd echt maximaal kunnen benutten.

Dank aan de klinische teams ketogeen dieet. Een mooi staaltje multidisciplinair werken. Dank aan het team uit Oosterhout om patiënten en ouders te informeren over het onderzoek en hun voor deelname naar Heeze te verwijzen. Dank aan Carly Jansen en Marion van Ool om patiënten en ouders te blijven motiveren om (gedurende de vaak lange tijd van het onderzoek) onderzoeksgegevens aan te leveren. Dank voor het helpen verzamelen van de data. Beste Marloes van der Star en Miranda 
Beeren, kinderartsen van het keto-team, hartelijk dank voor jullie hulp bij het analyseren en interpreteren van de vele gegevens uit de 'growth analyser' en de berg cholesterolwaardes.

Dank aan de onderzoeksverpleegkundigen voor hun onmisbare hulp bij het invoeren van de onderzoeksgegevens in databases. Beste Elly Vos, het was zo fijn om na de Tinnegieter tijd weer met je samen te werken. Ik kende de accuraatheid, stiptheid en flair waarmee je werkt. Niets mag aan het toeval overgelaten worden; bij twijfel overleg. De gegevens waren bij jou in goede handen en dat was een grote geruststelling.

Dank aan de medewerkers van het klinisch chemisch laboratorium.

Dr. J. Hulsman, beste Jacques, als gedreven klinisch chemicus, ervaren onderzoeker en voorzitter van de METC en de geneesmiddelengroep leerde ik je kennen. Dit was toen $i k$, nog groen achter de oren, meteen na mijn opleiding in Kempenhaeghe kwam werken. Je hebt me de weg gewezen in onderzoeksland. Ik heb bewondering voor je gedrevenheid en de correctheid waarmee je alles goed geregeld wilde hebben en zéker voor alle personen die naast patiënt ook proefpersoon wilden zijn. Met het artikel over CCK-8 en leptine zijn we een nieuwe weg ingeslagen. Ik hoop dat je er trots op kan zijn. Dank voor alles.

Dr. P. Verschuure, beste Pauline, dank voor je hulp bij alle bepalingen in het klinisch chemisch laboratorium van Kempenhaeghe. Het was meermaals 'scherp aan de wind' naar de door ons gestelde deadlines toe. Het CCK-8 verhaal was een ware uitdaging die we met succes hebben afgerond.

Adriaan Schellekens en Olga Hendriks dank voor het registreren van de laboratoriumresultaten van alle personen die meededen aan het ketogeen dieet onderzoek.

Dank aan alle medewerkers van de gedragswetenschappelijke dienst en van de KNFafdeling van Kempenhaeghe. In het bijzonder wil ik Debby Schrans en Nora de la Parra bedanken voor hun inzet bij het verzamelen en verwerken van alle gegevens van de neuropsychologische onderzoeken van de observationele studie. Beste Dominique IJff, hartelijk dank voor je waardevolle hulp bij het verwerken van de onderzoeksgegevens van het kosteneffectiviteit onderzoek. Succes gewenst met de laatste loodjes van jouw promotie.

Beste Ankie van Ojen, als secretaresse stond je mee aan de wieg van het klinisch traject en de onderzoeken over ketogeen dieet. Wel nu, je kindje is volwassen geworden. Veel dank voor de ondersteuning van de patiëntenzorg en het onderzoek. Je hulp bij het herwerken van het onderzoeksprotocol bij een volgend amendement was van onschatbare waarde. Het is ons nooit duidelijk geworden waarom de lay-out steeds vreemde wijzigingen creëerde maar het kwam uiteindelijk allemaal goed. 
Inmiddels worden alle logistieke uitdagingen vlot gestroomlijnd door Linda Linssen. Het KD programma is hiermee in handen van een waardig en deskundig opvolgster.

Beste Ton Smit ${ }^{\dagger}$, als meesterkok heb je je hulp aangeboden. Daarnaast was je kennis als diëtist met belangstelling voor voedseltechnologie gigantisch. Je ideeën waren creatief en innovatief. Je zorgde voor een grote hoeveelheid nieuwe recepten met een culinair tintje. Je maakte nieuwe bereidingen van de vetemulsie. Je zag het ketogeen dieet als een gezinsgebeuren dat vlotjes diende te verlopen zonder tien verschillende kookpotten op het fornuis. Je voorliefde voor verse en pure producten was groot. Ik zal je uitspraak "Gaan we koken of gaan we assembleren" nooit vergeten. Je hebt me doen nadenken over voeding en ervoor gezorgd dat ik zelf bewuster en gezonder eet. Dank voor alles.

Beste Willem Berfelo en Gerhard Blaauw, ik waardeer het in hoge mate dat jullie nieuwe gerechten ontwikkelen voor het gemodificeerde Atkins dieet. Met veel passie en plezier wordt de website gevuld. Straks heb ik weer wat meer tijd, laat maar weten wanneer jullie een 'proefkonijn' nodig hebben.

Beste Mirjam Brennand, hartelijk dank voor het lezen en corrigeren van de Nederlandse teksten.

Beste Irene Gijselhart, wat heb je ongelofelijk veel voor me gedaan. Opvragen van artikelen, adviseren hoe je niet-alledaagse referenties vermeldt, bouwen van een Endnote bibliotheek, assisteren bij Endnote complicaties; geen enkele vraag was je teveel. En dat met een tempo waaraan de hele gezondheidszorg een voorbeeld kan nemen.

Beste HTA-specialisten, van QALYs, IKERs en Markov modellen had ik nauwelijks iets gehoord voor we het onderzoeksvoorstel voor het kosteneffectiviteit onderzoek begonnen te schrijven. Hiermee is voor mij een nieuwe wereld opengegaan. Een andere manier van gegevens benaderen. Ik heb jullie één voor één leren kennen als zeer deskundige, enthousiaste en positief ingestelde personen met de nodige humor en relativeringsvermogen. Ik kijk met veel plezier en bewondering terug naar alle overlegmomenten.

Prof. dr. S.M.A.A. Evers, beste Silvia, hartelijk dank voor je waardevolle adviezen en geruststellende woorden.

Dr. D van Helvoort-Postulart, beste Debby, bijzonder veel dank voor je hulp bij het schrijven van het onderzoeksprotocol en de subsidie aanvraag van het kosteneffectiviteit onderzoek ketogeen dieet. Met veel kennis van zaken en gedreven door de wens om iets te doen voor het epilepsieonderzoek heb je je belangeloos op een zeer deskundige en zeer aangename manier ingezet. Daar heb ik veel respect en waardering voor.

Beste Reina de Kinderen en Ben Wijnen, hartelijk dank voor alles wat ik van jullie heb geleerd. Succes met je verdere carrière, Reina en met jouw promotie, Ben. 
Dank aan alle co-auteurs voor hun waardevolle bijdrages aan de artikelen van dit proefschrift. Het was een plezier om met jullie samen te werken.

Dank aan alle collega's die patiënten hebben verwezen voor deelname aan het onderzoek.

Dr. M. Chatrou en drs. N Geurts raad van bestuur, prof. dr P.A.J.M. Boon directeur Onderzoek \& Ontwikkeling, mevrouw A.M.J. de Ruiter en dr. A.J.A. de Louw directeurs Academisch Centrum voor Epileptologie wil ik bedanken voor het bieden van de mogelijkheid om klinische werk te combineren met onderzoek doen. Het geeft veel voldoening om als medisch specialist onderzoeksresultaten meteen te kunnen benutten om de dagelijkse patiëntenzorg te verbeteren.

Dr. S.C.M. Ebus en dr. R.J.A. de Kinderen, beste Saskia en Reina, jullie zijn de beste paranimfen die ik me had kunnen wensen. Wat een energie en positiviteit stralen jullie uit! Jullie promoveerden allebei in mei 2015, vijf dagen na elkaar. We zaten in hetzelfde schuitje en dat geeft een bijzondere band.

Beste Reina, ik heb heel veel van je geleerd. Van hoe zet je een optimaal werkbare database op in SPSS, tot hoe klasseer je massa's documenten en vooral hoe vind je deze ook weer snel terug $(-)$. Ik zal me je deskundigheid, je vrolijkheid en de vlotheid waarmee je met iedereen omgaat blijven herinneren. Ik denk met plezier terug aan de vele uren die we samen in het gebouw Den Boondert hebben doorgebracht.

Beste Saskia, aan jouw zijde mocht ik als paranimf de promotiezaal alvast van de andere kant bekijken. Wat deed jij het goed en wat voelde ik me zenuwachtig. Ook van jou heb ik veel geleerd. De vasthoudendheid en precisie waarmee je de gegevens van de 24-uurs EEG's voor ons gemeenschappelijk artikel analyseerde, is indrukwekkend. We leerden allebei om ons niet meer te laten tegenhouden door onzekerheid en details. Je hulp en steun tijdens het laatste jaar van mijn promotietraject verdienen een bijzonder woord van dank. Meerdere keren hebben we afgesproken om de stand van zaken door te nemen. Met de goede adviezen die je gaf, kon ik weer met een sprong vooruit. Op eigen initiatief heb je de poli in Sittard waargenomen. Ik waardeer je inzet als collega en als paranimf enorm.

Liefste Joren en Bram, jullie zijn mijn kracht en mijn zwakte tegelijkertijd. Joren, bedankt voor je woorden "mama, ondanks dat je veel werkt, heb je toch nog veel tijd voor ons". Bram, bedankt voor je woorden "mama, je zit teveel achter je computer". Deze combinatie hielp me om het juiste evenwicht te bewaren. Het doet me plezier te zien dat jullie allebei ook gepassioneerd zijn om gedreven door leergierigheid kennis te verzamelen. Ik hoop dat dit jullie je leven lang veel plezier blijft opleveren.

Liefste Karel, we hebben allebei "ja” gezegd op de vraag of promoveren er ook nog bij kon. We beseften allebei niet hoe groot de investering zou zijn. Dank voor je hulp bij onwillige documenten, figuren en tabellen. Dank om het boekje door te lezen en de 
fouten eruit te halen die bij mij door de mazen van het net waren geglipt. Dank voor de vele klussen die je me uit handen nam. Het is fijn om het weekend te kunnen beginnen met een gevulde koelkast. Soms zou promoveren eeuwig mogen duren. Het doel is bereikt en dat mag gevierd worden.

Liefste ouders, dank dat ik heb mogen studeren waar m'n hart naar uitging. Ma, dank voor het opvangen van de kinderen op die vele momenten dat ik aan het werk ben. Te weten dat ze in goede en liefdevolle handen zijn, geeft me rust en plezier in mijn werk. Pa, je kan het niet meer meemaken maar ik weet dat je zou stralen van trots als je m'n boekje had kunnen vast nemen. Je motto "carpe diem/pluk de dag" wil ik bij deze aan iedereen die dit dankwoord leest, meegeven.

Tot slot dank aan alle professionals, familieleden, kennissen en vrienden die me hebben geholpen en gesteund maar niet met naam werden genoemd. 
List of publications 


\section{List of publications}

\section{Peer-reviewed international publications}

Aldenkamp AP, Arends JBAM, Bootsma HP, Diepman L, Hulsman JARJ, Lambrechts DAJE, Leenen L, Majoie HJM, Schellekens A, de Vocht J.

Randomized double-blind parallel-group study comparing cognitive effects of a Lowdose lamotrigine with valproate and placebo in healthy volunteers.

Epilepsia 2002;43(1):19-26

Bootsma HP, Coolen F, Aldenkamp AP, Arends J, Diepman L, Hulsman J, Lambrechts D, Leenen L, Majoie M, Schellekens A, de Krom M.

Topiramate in clinical practice: long-term experience in patients with refractory epilepsy referred to a tertiary epilepsy center.

Epilepsy \& Behavior 2004;5(3):380-7

Lambrechts DAJE, Sadzot B, van Paesschen W, van Leusden JA, Carpay J, Bourgeois P, Urbain E, Boon PAJM.

Efficacy and safety of Levetiracetam in clinical practice: Results of the SKATE trial from Belgium and the Netherlands.

Seizure 2006;15(6):434-42

Bootsma HP, Aldenkamp AP, Diepman L, Hulsman J, Lambrechts D, Leenen L, Majoie $M$, Schellekens A, de Krom M.

The effect of antiepileptic drugs on cognition: patient perceived cognitive problems of topiramate versus levetiracetam in clinical practice.

Epilepsia 2006;47 supp/ 2:24-7

Bootsma HP, Ricker L, Diepman L, Gehring J, Hulsman J, Lambrechts D, Leenen L, Majoie M, Schellekens A, de Krom M, Aldenkamp AP.

Levetiracetam in clinical practice: long-term experience in patients with refractory epilepsy referred to a tertiary epilepsy center.

Epilepsy \& Behavior 2007;10(2):296-303

Bootsma HP, Vos AM, Hulsman J, Lambrechts D, Leenen L, Majoie M, Savelkoul M, Schellekens A, Aldenkamp AP.

Lamotrigine in clinical practice: long-term experience in patients with refractory epilepsy referred to a tertiary epilepsy center.

Epilepsy \& Behavior 2008;12(2):262-8 
Bootsma HP, Ricker L, Diepman L, Gehring J, Hulsman J, Lambrechts D, Leenen L, Majoie M, Schellekens A, de Krom M, Aldenkamp AP.

Long-term effects of levetiracetam and topiramate in clinical practice: a head-to-head comparison.

Seizure 2008;17(1):19-26

Bootsma HP, Ricker L, Hekster Y,Hulsman J, Lambrechts D, Majoie M, Schellekens A, de Krom M, Aldenkamp AP.

The impact of side effects on long-term retention in three new antiepileptic drugs.

Seizure 2009;18:327-331

Van Delft R, Lambrechts D, Verschuure P, Hulsman J, Majoie M.

Blood beta-hydroxybutyrate correlates better with seizure reduction due to ketogenic diet than do ketones in the urine.

Seizure 2010;19(1):36-9

de Kinderen R, Lambrechts D, Postulart D, Kessels A, Hendriksen J, Aldenkamp A, Evers S, Majoie M.

Research into the (cost-)effectiveness of the ketogenic diet among children and adolescents with intractable epilepsy: design of a randomized controlled trial.

BMC Neurology 2011;11:10

Lambrechts DA, Wielders LH, Aldenkamp AP, Kessels FG, de Kinderen RJ, Majoie MJ. The ketogenic diet as a treatment option in adults with chronic refractory epilepsy: efficacy and tolerability in clinical practice.

Epilepsy\& Behavior 2012;23(3):310-4

Lambrechts DA, Bovens MJ, de la Parra NM, Hendriksen JG, Aldenkamp AP, Majoie MJ.

Ketogenic diet effects on cognition, mood, and psychosocial adjustment in children.

Acta Neurol Scand 2013;127(2):103-8

Wegner I, Wilhelm AJ, Lambrechts DAJE, Sander JW, Lindhout D.

Effect of oral contraceptives on lamotrigine levels depends on comedication.

Acta Neurol Scand. 2014;129(6):393-8

Ebus SCM*, Lambrechts DAJE*, Herraets IJT, Majoie HJM, de Louw AJA, Boon PAJM, Aldenkamp AP, Arends JBAM. * Both authors contributed equally to this study.

Can an early 24-hour EEG predict the response to the ketogenic diet? A prospective study in 34 children and adults with refractory epilepsy treated with the ketogenic diet.

Seizure 2014;23(6):468-74 
de Kinderen R, Postulart D, Aldenkamp A, Evers S, Lambrechts D, de Louw A, Majoie $\mathrm{M}$, Grutters J.

Cost-effectiveness analysis of the ketogenic diet versus the vagus nerve stimulator and care as usual for the treatment of children with refractory epilepsy.

Epilepsy Research 2015;110;119-31

Lambrechts DAJE, de Kinderen RJA, Vles JSH, de Louw AJA, Aldenkamp AP*, Marian HJM Majoie*. * Both authors contributed equally to this study.

The ketogenic diet as a treatment option in refractory childhood epilepsy: a prospective study with 2 years follow-up.

Epilepsy \& behavior 2015;51:261-6

de Kinderen RJA, Lambrechts DAJE, Wijnen BFM, Postulart D, Aldenkamp AP, Majoie HJM, Evers SMAA.

An economic evaluation of the ketogenic diet versus care as usual in children and adolescents with intractable epilepsy: an interim analysis.

Epilepsia 2016;57(1):41-50

Lambrechts DAJE, de Kinderen RJA, Vles JSH, de Louw AJA, Aldenkamp AP, Majoie HJM. A randomized controlled trial of the ketogenic diet in refractory childhood epilepsy.

Acta Neurol Scand 2016 Mar 29. doi: 10.1111/ane.12592

\section{Submitted publications}

Lambrechts DAJE*, Brandt-Wouters E*, Verschuure P, Vles JSH, Majoie HJM. * Both authors contributed equally to this study.

A prospective study on changes in blood levels of cholecystokinin-8 and leptin in patients with refractory epilepsy treated with the ketogenic diet.

IJff DM, Postulart D, Lambrechts DAJE, Majoie HJM, de Kinderen RJA, Hendriksen JG, Evers SMAA, Aldenkamp AP.

Cognitive and behavioral impact of the ketogenic diet in children and adolescents with refractory epilepsy: a randomized controlled trial.

\section{National publications with referee system}

Rinkel G, Brouwers P, Lambrechts D.

Voor de praktijk: klinisch denken en beslissen in de praktijk. Een dirigent met epilepsie gevolgd door geheugenstoornissen.

Nederlands Tijdschrift voor Geneeskunde 1997;141(15):723-6

\section{Lambrechts DAJE.}

Begeleiding van zwangeren met epilepsie.

Epilepsie Periodiek voor professionals 2003;3:23-28 


\section{Lambrechts DAJE.}

Ketogeen dieet als behandeling van therapieresistente epilepsie.

Epilepsie Periodiek voor Professionals 2007; 22-24

Majoie M, Lambrechts D.

Evaluatie van het ketogene dieet als behandeling van therapieresistente epilepsie op kinderleeftijd.

Tijdschrift voor Neurologie \& Neurochirurgie 2009, vol 110, nr 4 p 204-206

Majoie HJM, Lambrechts DAJE.

De effectiviteit van het ketogeen dieet bij kinderen met het syndroom van LennoxGastaut. Journal scan van: Lemmon ME, Terao NN, Yu-Tze NG, et al.

Efficacy of the ketogenic diet in Lennox-Gastaut syndrome: a retrospective review of one institution's experience and summary of the literature. Developmental Medicine \& Child Neurology, 2012;54(5):464-8

Tijdschrift voor Neurologie en Neurochirurgie 2012; 113(2): 138-140

De methodologische kwaliteit van economische evaluaties.

$\mathrm{R}$ de Kinderen, S Evers, D Postulart, D Lambrechts, A Aldenkamp, M Majoie.

Epilepsie. Periodiek voor professionals 2012;10(1): 20-23

\section{Books}

Lambrechts D, Gerrits-Troost CM, Boomsma M.

Voeding bij epilepsie.

In: Carbasius Weber EC, Post GB, Swager TW

Informatorium voor Voeding \& Diëtetiek

Bohn Stafleu Van Loghum, Houten, XXVIb-1 tot 20 (suppl 59, 2005)

Lambrechts D, Gerrits-Troost CM, Boomsma M.

Voeding bij epilepsie (aug 2012).

In: Carbasius Weber EC, Post GB, Swager TW

Informatorium voor Voeding \& Diëtetiek. Houten, Bohn Stafleu Van Loghum.

Dorine van den Hurk, Danielle Lambrechts.

3.2. Het ketogene dieet bij therapie resistente epilepsie, GLUT1 en pyruvaatdehydrogenase deficiëntie in Dieet bij Metabole Ziekten (2011).

Uitgegeven door Carbasius Weber, van Veen en Visser, WKZ/UMC Utrecht, 3584 EA Utrecht.

ISBN 978-90-817457-0-3 


\section{Abstract publications}

Aldenkamp AP, Arends JBAM, Bootsma HP, Diepman L, Hulsman JARJ, Lambrechts DAJE, Leenen L, Majoie HJM, Schellekens A., Schlosser A, de Vocht J.

A randomized double-blind parallel-group study comparing cognitive effects of lamotrigine with valproate and placebo in healthy volunteers.

Epilepsia 2001;42(S2),150

Veendrick-Meckes M, Renier WO, Oei LT, Lambrechts D.

Levetiracetam therapy in children and adolescents with intractable epilepsy.

Epilepsia 2002;43(S8):37

Bootsma H, Coolen F, Leenen L, Lambrechts D, Hulsman J, Majoie M, Diepman L, Schellekens A, Arends J, Aldenkamp A.

Topiramate in an epilepsy centre; drug use evaluation study.

Epilepsia 2002; 43(S8):121

Bootsma HP, Coolen F, Aldenkamp A, Arends J, Hulsman J, Lambrechts D, Leenen L, Majoie $M$, Schellekens $A$, de Krom $M$.

Topiramate in clinical practice: long term experience of patients with refractory epilepsy referred to a tertiary epilepsy centre.

Epilepsia 2003; 44 (S8):117-118

Lambrechts DAJE, Boon PAJM.

Open-label, multi-centre, community-based trial on the safety and efficacy of levetiracetam as add-on therapy in adults with uncontrolled partial epilepsy in the Benelux region.

J Neurol 2004;251(Supplement 3):III/9

Flipsen-ten Berg K, van Oppen ACC, Wentges-van Holthe JML, Schielen PCJI, Lambrechts DAJE, Galjaard RJ, Lindhout D.

Neuronal tube defects associated with prenatal exposure to antiepileptic drugs.

Reproductive Toxicology; 2007(24)1:60-61

Ebus S, Ossenblok P, Lambrechts D, Arends J, Krijn R, Majoie M, Boon P.

Do changes in interictal epileptiform activity or EEG background correlate to outcome in patients treated with ketogenic diet ?

Epilepsia 2007;48;56:92-93 
Theuniszen E, Ebus S, Lambrechts D, Krijn R, Arends J, Boon P, Ossenblok P.

The ketogenic diet and EEG-background changes.

Epilepsia 2011;52(Suppl 6) 161

de Kinderen RJA, Postulart D, Evers SMAA, Lambrechts DAJE, Aldenkamp AP, Majoie HJM.

Economic evaluations of treatments for intractable epilepsy: a methodological review. Gaceta Sanitaria 2012, vol. 26 - Especial Congreso 2

Veendrick-Meekes MJBM, Lambrechts DAJE, van Erp G.

Identifying SCN1A mutations and treatment options in adult patients.

Epilepsia 201354 (Supp/ 3) 242-243 
Curriculum Vitae 


\section{Curriculum Vitae}

Danielle Lambrechts werd op 24 april 1967 geboren in Hasselt (België).

Danielle doorliep de middelbare school op het Humaniora Zusters kindheid Jesu (het huidige Virga Jesse College) in Hasselt. Ze studeerde in Hasselt en in Leuven geneeskunde. In 1993 behaalde ze aan de Katholieke Universiteit Leuven (KUL) het diploma 'doctor in de genees-, heel- en verloskunde'. Fascinatie voor de keuzestage neurologie aan het begin van de huisartsenopleiding leidde al snel tot een koerswijziging. In 1999 behaalde Danielle het diploma

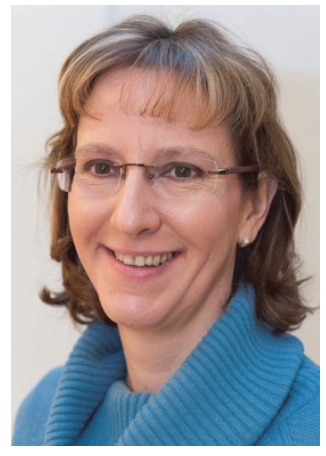
'medisch specialist in de neurologie' aan de KUL. Tijdens haar opleiding tot neuroloog verdiepte ze zich in het academisch ziekenhuis in Utrecht in kinderneurologie en in het AZ Sint-Jan in Brugge in klinische neurofysiologie.

Sinds 1999 werkt Danielle als neuroloog in het Academisch Centrum voor Epileptologie Kempenhaeghe/Maastricht UMC+. Haar aandachtsgebieden zijn ketogeen dieet, geneesmiddelenonderzoek en specifieke aan vrouw-zijn gerelateerde aspecten van epilepsie. Van 2000 tot 2010 was ze lid van de medisch ethische toetsingscommissie. Sinds 2002 is ze programmaleider van het behandeltraject ketogeen dieet en actief lid van de landelijke werkgroep ketogeen dieet.

In de afgelopen jaren combineerde Danielle haar taken binnen de patiëntenzorg met promotieonderzoek. De resultaten van dit onderzoek zijn beschreven in dit proefschrift.

Danielle woont in Zonhoven (België) met haar echtgenoot Karel Van Gansberghe en hun zonen Joren (14 jaar) en Bram (12 jaar). 
